\title{
Model predictive control of trailing edge flaps on a wind turbine blade
}

\author{
Castaignet, Damien Bruno
}

Publication date:

2011

Document Version

Publisher's PDF, also known as Version of record

Link back to DTU Orbit

Citation (APA):

Castaignet, D. B. (2011). Model predictive control of trailing edge flaps on a wind turbine blade. Technical University of Denmark. Risø National Laboratory for Sustainable Energy.

\section{General rights}

Copyright and moral rights for the publications made accessible in the public portal are retained by the authors and/or other copyright owners and it is a condition of accessing publications that users recognise and abide by the legal requirements associated with these rights.

- Users may download and print one copy of any publication from the public portal for the purpose of private study or research.

- You may not further distribute the material or use it for any profit-making activity or commercial gain

- You may freely distribute the URL identifying the publication in the public portal

If you believe that this document breaches copyright please contact us providing details, and we will remove access to the work immediately and investigate your claim. 


\section{Model predictive control of trailing edge flaps on a wind turbine blade.}

Damien Castaignet 
Technical University of Denmark

National Laboratory for Sustainable Energy, Wind Energy Department Frederiksborgvej 399, DK-4000 Roskilde, Denmark

Phone +4546774677

risoe@risoe.dtu.dk

www.risoe.dtu.dk 


\section{Summary}

Trailing edge flaps on wind turbine blades have been investigated for several years. Aero-servoelastic simulations carried out with different simulation tools, trailing edge flaps configurations and controller designs proved that trailing edge flaps are a suitable solution for reducing some of the wind turbine fatigue and extreme loads. This potential was confirmed with wind tunnel tests made on blade sections with trailing edge flaps and on a scaled two-bladed wind turbine in a wind tunnel. The work presented in this thesis includes a full-scale test run on a Vestas V27 wind turbine equipped with three trailing edge flaps on one blade, located on the Risøcampus in Roskilde, Denmark.

This thesis is divided into three parts: the controller design, results from simulations, and results from the experiments.

The trailing edge flaps controller designed for this project is based on a frequency-weighted model predictive control, tuned in order to target only the flapwise blade root loads at the frequencies contributing the most to blade root fatigue damage (the $1 \mathrm{P}, 2 \mathrm{P}$ and $3 \mathrm{P}$ frequencies), and to avoid unnecessary wear and tear of the actuators at high frequencies. A disturbance model consisting in periodic disturbances at the rotor speed harmonic frequencies and a quasi-steady input disturbance is aggregated to an analytical model of a spinning blade with trailing edge flaps.

Simulations on a multi-meagawatt wind turbine show the potential of the trailing edge flaps to reduce the flapwise blade root fatigue loads by $23 \%$, but also the main shaft and the tower fatigue loads by up to $32 \%$. Extreme loads during normal production also benefit from the trailing edge flaps.

At last, the same controller was run on the Vestas V27 wind turbine located at the Ris $\varnothing$ campus of the Technical University of Denmark, in Roskilde, Denmark. One blade of the turbine was equipped with three independent trailing edge flaps. In spite of the failure of several sensors and actuators, the test of the trailing edge flaps controller described in this thesis showed a consistent flapwise blade root fatigue load reduction. An average of $14 \%$ load reduction was achieved during a 38 minute test.

However, the experiment also highlighted the weaknesses of the controller. The trailing edge flap controller should be made more adaptive in order to cope with the very different wind conditions that can be expected on-site.

The contributions of the thesis have been documented in a series of scientific papers. The papers 
form the main part of this thesis. 


\section{Resumé}

Bevægelige bagkanter på vindmøllevinger, såkaldte flaps, har i de seneste årti været genstand for betydelig forskning. Gennem aero-servo-elastiske simuleringer med forskellige beregningsværkt $\varnothing$ jer og styringsrutiner er det på vist at udvalgte flapkonstruktioner kan reducere bå de udmattelses- og ekstremlaster på vindmøller. Dette potentiale har ligeledes været bekræftet med vindtunnelforsøg på vingesektioner og på en nedskaleret mølle med to vinger. Arbejdet, der præsenteres i denne afhandling, indeholder en fuldskalatest på en Vestas V27 vindmølle på Ris $\varnothing$ ved Roskilde udstyret med tre bagkantsflaps på den ene vinge.

Afhandlingen er opdelt i tre dele: Design af flapstyring, resultater fra simuleringer og resultater fra fuldskalatesten.

Den præsenterede flapstyring er en frekvens-vægtet model-prediktiv styring, som er indstillet til at reducere flapvise vingerods-udmattelseslaster. Dette opnås ved kun at behandlede de frekvenser som bidrager mest til de flapvise vingerods-udmattelseslaster (1P, $2 \mathrm{P}$ og $3 \mathrm{P}$ frekvenserne). Der undgås et unødigt slid på flap-aktuatorerne ved at forsøge at behandle højere frekvenser. En beskrivelse af forstyrrelserne fra både det rotorhastighedsperiodiske og det kvasistatiske er samlet til en analytisk prediktions-model for simulering af gensvar af en roterende vinge med bagkantsflapper i et fluktuerende vindfelt.

Simuleringer på en multimegawatt vindmølle viser de bevægelige bagkanters evne til at reducere de flapvise vingerods-udmattelseslaster med $23 \%$ og ligeledes udmattelseslaster i hovedaksel og tårnet med op til 32\%. Ekstremlaster under normal drift reduceres også med bevægelige bagkanter.

Afslutningsvis er flapstyringen afprøvet eksperimentelt på Vestas V27 møllen med de tre flaps på den ene vinge. På trods af komplikationer med svigt af sensorer og aktuatorer blev der opnået en entydig reduktion af de flapvise vingerods-udmattelseslaster. I en sammenhængende periode på 38 minutter blev der opnået en gennemsnitlig reduktion på $14 \%$.

Eksperimentet viste imidlertid også nogle svagheder ved styringsrutinen for de bevægelige bagkanter. Styringen skal i praksis gøres mere adaptiv for at tilpasse sig til de forskellige variationer i vindtilstande, der optræder i virkeligheden.

De enkelte dele af afhandlingen er blevet søgt publiceret i en række videnskabelige artikler ved konferencer og i tidsskrifter. Disse artikler udgør en hovedpart af afhandlingen. 


\section{Preface and acknowledgements}

This Ph.D. thesis was prepared at DTU Wind Energy, Technical Univerity of Denmark, Ris $\varnothing$ campus, Roskilde, Denmark, while I was employed as an industrial PhD student by Vestas Wind Systems A/S, Global Research \& Innovation. The work was carried out in the period December 2008 - February 2012. The supervisors were Senior Scientist Thomas Buhl (DTU Wind Energy), Associate Professor Niels Kjølstad Poulsen (DTU Informatics) and Senior Specialist Jens Jakob Wedel-Heinen (Vestas Wind Systems A/S). The project was partially funded by the Danish National Advanced Technology Foundation (Højteknologiefonden) through the ATEF project (grant 028-2007-3).

I am grateful to my supervisors Thomas Buhl, Niels Kjølstad Poulsen and Jens Jakob WedelHeinen for their invaluable guidance, advice and help during those three years. Special thanks also to my Vestas colleagues in the Ris $\varnothing$ office for making this office such a nice place to work in: Tim for sharing his $\mathrm{PhD}$ experience, his good karma, and for his talent for writing patent applications and drinking beers, Rolf \& Martin the Swedes for their boiled eggs, and the West side crew, Jakob, Niels and Karsten, for their introduction to the danish culture and exotic cuisine. I would also like to acknowledge Kelvin and Ian for their permanent support and help especially on Vestas controls and on MPC.

Model predictive control of trailing edge flaps is nice, but hanging out with friends is even nicer. Thanks to my "Danish" friends in Copenhagen for improving my social life and to the 1C crew of polaaars for providing me with fruitful readings.

Finaly, I want to thank my parents and my relatives, especially Mamie, for 29 years of happiness, and Adèle and Mateusz for promoting wind energy in France... 


\section{Papers included in the thesis}

A Damien Castaignet, Niels Kjølstad Poulsen, Thomas Buhl and Jens Jakob Wedel-Heinen. Model Predictive Control of Trailing Edge Flaps on a Wind Turbine blade. In proceedings of American Control Conference, 2011, San Francisco, California. Published.

B Damien Castaignet, Ian Couchman, Niels Kjølstad Poulsen, Thomas Buhl and Jens Jakob Wedel-Heinen. Frequency-Weighted Model Predictive Control of Trailing Edge Flaps on a Wind Turbine Blade. IEEE transactions on Control Systems Technology. Submitted.

C Damien Castaignet, Thomas Buhl, Niels Kjølstad Poulsen and Jens Jakob Wedel-Heinen. Trailing edge flaps impact on fatigue and extreme loads in power production. In proceedings of EWEA 2011, Brussels, Belgium. Published.

D Damien Castaignet, Leonardo Bergami, Thomas Buhl, Niels Kjølstad Poulsen and Jens Jakob Wedel-Heinen. Robustness assessment of a flap controller with two alternative aeroelastic simulation tools. Wind Energy, 2012. Submitted.

E Damien Castaignet, Jens Jakob Wedel-Heinen, Taeseong Kim, Thomas Buhl and Niels Kjølstad Poulsen. Results from the first full scale wind turbine equipped with trailing edge flaps. In proceedings of the $28^{\text {th }}$ AIAA Applied Aerodynamics Conference, 2010, Chicago, Illinois. Published.

F Damien Castaignet, Thanasis Barlas, Thomas Buhl, Niels Kjølstad Poulsen, Jens Jakob Wedel-Heinen, Niels Anker Olesen, Christian Bak and Taeseong Kim. Full-scale test of Trailing Edge Flaps on a Vestas V27 wind turbine. Active load reduction and system identification. Wind Energy, 2012. Submitted.

Besides these papers, this thesis refers to two Vestas proprietary technical reports with restricted access [23, 24]. 


\section{Contents}

Summary i i

Resumé

\begin{tabular}{lll}
\hline Preface and acknowledgements & v
\end{tabular}

Papers included in the thesis vii

\begin{tabular}{lll|}
\hline & Introduction & 1
\end{tabular}

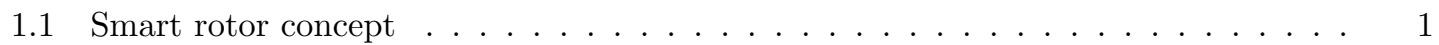

1.2 Model Predictive Control $\ldots \ldots \ldots \ldots$. . . . . . . . . . . . . . 4

$1.3 \quad$ ATEF project and V27 demonstrator . . . . . . . . . . . . . . . . 6

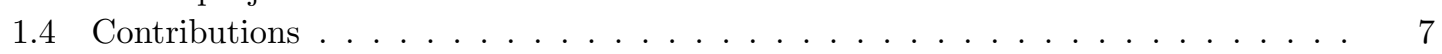

1.5 Outline of the thesis $\ldots \ldots \ldots \ldots \ldots \ldots$

\begin{tabular}{|lll|}
\hline 2 & Control system design & 9
\end{tabular}

2.1 Wind turbine loads . . . . . . . . . . . . . . . . . . . . . 9

2.2 Wind speed estimator and gain scheduling . . . . . . . . . . . . . . . . . . . 11

2.3 Model Predictive Control $\ldots \ldots \ldots \ldots \ldots$. . . . . . . . . . . . . . . 14

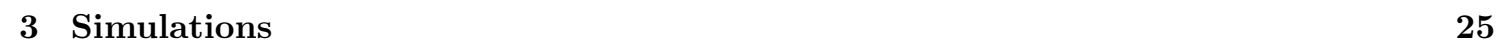

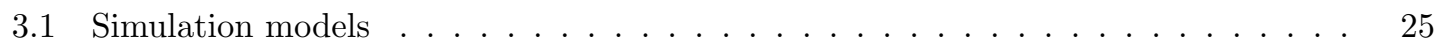

3.2 Trailing edge flap controller implementation . . . . . . . . . . . . . . . . . . 27

$3.3 \quad$ MPC performance, fatigue and extreme loads . . . . . . . . . . . . . . . 27

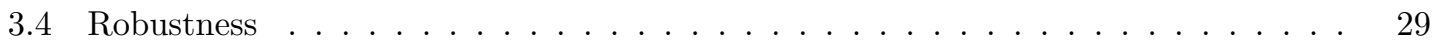

\begin{tabular}{lll}
\hline 4 & Experiments & 31
\end{tabular}

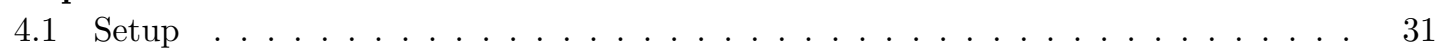

4.2 Implementation . . . . . . . . . . . . . . . . . . . . . . . . . . 35

4.3 Results. . . . . . . . . . . . . . . . . . . . . . . . . . . 37

\begin{tabular}{|lll}
5 & Conclusions and future development & 43
\end{tabular}

\begin{tabular}{ll}
\hline Bibliography & 48
\end{tabular}

\begin{tabular}{ll}
\hline Papers & 48
\end{tabular}

\begin{tabular}{|lll}
\hline A Model Predictive Control of Trailing Edge Flaps on a Wind Turbine blade & 50
\end{tabular} 
B Frequency-Weighted Model Predictive Control of Trailing Edge Flaps on a Wind Turbine Blade $\quad 58$

\begin{tabular}{|lll} 
C Trailing edge flaps impact on fatigue and extreme loads in power production & 74
\end{tabular}

D Robustness assessment of a flap controller with two alternative aeroelastic sim$\begin{array}{lc}\text { ulation tools } & 84\end{array}$

E Results from the first full scale wind turbine equipped with trailing edge flaps 98

F Full-scale test of Trailing Edge Flaps on a Vestas V27 wind turbine. Active load $\begin{array}{lll}\text { reduction and system identification } & 108\end{array}$ 
CHAPTER 1

\section{Introduction}

\subsection{Smart rotor concept}

The wind energy market grew significantly in the last decade, with the wind energy contribution to the global energy market being larger and larger. Wind energy research has focused in improving the integration of wind turbines in the electrical grids and in reducing the cost of wind energy, in an effort to increase even more the share of wind energy in the world.

Increasing the rotor size, and thus the swept area, for the same drive train and rated power, has been one of the solutions to decrease the cost of wind energy, especially at low turbulence sites. The leading wind turbine manufacturers now market turbines with rotor diameters over $100 \mathrm{~m}$ and nominal power from 3 to $6 \mathrm{MW}$. Increasing the rotor area increases the energy harvested by the rotor, but also increases significantly the fatigue and extreme loads of the wind turbine. Research is now being focused in reducing the wind turbine loads, by using new control strategies, new sensors and new actuators.

\subsubsection{Pitch control for load alleviation}

The loads acting on the wind turbine are a combination of aerodynamic, gravity, centrifugal and inertia loads. They originate in the wind conditions, such as the wind shear, the wind turbulence or the wind dissymmetry, or in control induced phenomena like a grid loss or an emergency shutdown etc. [21, 43, 4].

This work focuses on pitch regulated wind turbines, where the blades are pitched out of the wind to decrease the energy capture. Stall regulated turbines are not considered.

Pitch regulated wind turbines can alleviate some of those loads by using the pitch actuators not only to perform power control, but also for load alleviation. Cyclic pitch and individual pitch control are already implemented in some of the commercial wind turbines. 
Cyclic pitch control originated in the rotorcraft community. It consists in pitching the three blades with a 120 degree phase shift to alleviate the $1 \mathrm{P}$ and higher harmonic loads. The $1 \mathrm{P}$ loads are loads occuring at the $1 \mathrm{P}$ frequency, corresponding to one event per rotor revolution. The $2 \mathrm{P}$ and $3 \mathrm{P}$ frequencies are twice and thrice higher. In the case of positive wind shear, where the wind speed is higher at the rotor top than at the rotor bottom, cyclic pitch can reduce the main shaft tilt moment by pitching the blade out of the wind when it reaches the rotor top, and pitching it in the wind at the rotor bottom.

The natural extension to cyclic pitch is individual pitch control where each blade can pitch independently of the two other blades. It is one of the most advanced active control to alleviate loads implemented in today's turbines [18, 19, 40, 51, 30. Both cyclic and individual pitch controls proved to be succesfull in reducing fatigue loads in the blades (10 to 20\%), as well as in the main shaft and in the tower. However, cyclic and individual pitch controls have known limitations. The load reduction is limited by the pitch actuator ability to pitch blades which weigh several tonnes. Most of today's actuators bound the pitch controller to target the 1P loads only. Cyclic and individual pitch control also require a higher pitch activity which wear and tear both the pitch actuators and the blade bearings. Problems also raise with modern blades which are longer and less stiff in torsion and can not tolerate fast pitch actuation speeds.

\subsection{2 "Smart" rotor}

Because of the limitation of cyclic and individual pitch control, research have focused during the last decade on more advanced methods to reduce further the wind turbine loads. Both passive and active load reduction devices are being researched and tested.

\subsubsection{Passive load control}

Passive load control concepts have the advantage of not requiring any extra sensor and actuator. This is of great importance for wind turbine manufacturers and owners when considering maintenance and operating expenses (OPEX). The two most researched rotor passive load control concepts are twist-bending coupled blades and swept blades.

Twist-bending coupled blades consist in coupling the bending of the blade with its spanwise twist [49, 22], for example by laying the composite layers of the blade with an angle with respect to the elastic center line of the blade. When the blade bends flapwise, the twist-bending coupling twists the blade in or out of the wind, which tends to reduce the amplitude of the flapwise bending moment of the blade. This method reduces both fatigue and extreme loads.

In swept blades, the aerodynamic center of the airfoils is moved further from the blade axis, so that an extra aerodynamic loading of the blade result in the twist of the blade [10, 28. Backward swept blades (pitch to feather) decrease the flapwise blade root fatigue loads, while forward swept blades increase them. Drawbacks of swept blades are an increase of the edgewise loads as well as the blade pitch moment, and a small reduction of the annual energy production. Their manufacturing and transport can also be an issue. 


\subsubsection{Active load control}

In order to reduce even further and in a more efficient way the loads on the rotor, several concepts of "smart" rotors have been investigated in the litterature. A "smart" rotor consists in distributed sensors (accelerometers, strain gages, Pitot tubes, pressure tabs etc.) and actuators (trailing edge flaps, microtabs [56, boundary layer suction or blowing jets, plasma actuators etc.) along the blades. This thesis only deals about trailing edge flaps. Barlas and van Kuik 12 wrote a detailed overview of the different "smart" rotor concepts being researched.

Trailing edge flaps are probably the most studied actuators within the "smart" rotor concept and have been thoroughly investigated for several years now [20]. Research on trailing edge flaps on wind turbine blades ranges from simulations and modeling to wind tunnel tests on 2D blade airfoils [7, 11, 59] and on a 2-bladed scaled turbine [58, and a full scale test on a Vestas V27 wind turbine presented with this thesis.

Basualdo [13] and Buhl et al. [20] showed the potential of trailing edge flaps to alleviate flapwise blade root fatigue loads by running $2 \mathrm{D}$ aeroelastic simulations. At the same time, Troldborg [55] studied the influence of parameters like the trailing edge flap shape and size with CFD simulations in order to optimise the flap design for wind turbine applications. Andersen et al. 8], Gaunaa 27] and Bergami and Gaunaa [17] developed an analytical model of the unsteady aerodynamic force distribution on an airfoil with variable camberline, based on the thin airfoil theory [53, 41]. This model was implemented in HAWC2 and used by Andersen et al. 9] to run 3D aeroelastic simulations of the $5 \mathrm{MW}$ NREL reference turbine [37. A 25\% flapwise blade root fatigue load reduction was achieved.

The ultimate goal of trailing edge flaps is to reduce the cost of wind energy. Berg et al. [15] showed that trailing edge flaps could lead to a 5 to $9 \%$ cost of energy reduction. Simulations were run with the FAST and CurveFAST aero-sero-elastic simulation codes.

Some wind tunnel tests were then performed at DTU Wind Energy, Technical University of Denmark. Andersen et al. 7] and Bak et al. [11] tested in a wind tunnel both open loop and closed loop controls on the Ris $\varnothing$-B1-18 wind turbine airfoil equipped with a piezo electric active trailing edge flap. Closed loop controls are based on the Pitot tube measurements and on the pressure difference between the suction and the pressure side at the airfoil leading edge. Van Wingerden et al. 59] also performed wind tunnel tests at Delft University of Technology on a scaled rotor blade equipped with two trailing edge flaps. At last, van Wingerden et al. [58] realised a wind tunnel test on a two-bladed scaled turbine. They reduced the variance of the flapwise blade root loads by $90 \%$.

Various controllers for trailing edge flaps, using different control theories and sensors, have been investigated. Behrens and Jun Zhu [14] simulated with a CFD code a controller based on the trailing edge flap hinge moment only. Lackner et al. 38 designed a PID Individual Flap Control based on the Individual Pitch Control scheme, using the Coleman transformation to make the system linear time invariant. This controller requires balde root strain gages and a rotor azimuth position sensor. Van Wingerden et al. [59, 58, used subspace system identification to fine tune the PD controller used in their wind tunnel test, and developed a feedback controller based on $\mathcal{H}_{\infty}$-loop shaping combined with a fixed-structure feedforward control which they succesfuly tested on the scaled turbine in a wind tunnel. Rice et al. [50 focused on a robust and distributed control in order to ensure stability of the controller despite non linearities and model mismatch. At last, Wilson et al. 60 designed PD feedback controllers based on tip deflection or tip deflection rate, and showed a decrease in the standard deviation of the flapwise blade root moments. 


\section{$1.2 \quad$ Model Predictive Control}

Model Predictive Control (MPC) [4, 42] is an advanced control theory that has been used in the process industry since the 1980s. In [45, Mayne et al. give the following definition: "Model predictive control (MPC) or receding horizon control (RHC) is a form of control in which the current control action is obtained by solving on-line, at each sampling instant, a finite horizon open-loop optimal control problem, using the current state of the plant as the initial state; the optimization yields an optimal control sequence and the first control in this sequence is applied to the plant."

The following introduction to model predictive control (Figure 1.1) is restricted to the basic formulation of linear state-space nominal model predictive control.

The following discrete time linear invariant system is considered:

$$
\begin{aligned}
x(k+1) & =A x(k)+B u(k)+G d(k) \\
z(k) & =C x(k)
\end{aligned}
$$

where $x$ is the state of the system, $u$ is the control input, $d$ is the measurable disturbance and $z$ the control output. The control objective is to minimise, at each time step $k$, the quadratic cost $\Psi(k)$ subject to some constraints on the inputs $u \in \mathbb{U}$.

A basic quadratic cost consists in costs on both the inputs and the outputs:

$$
\begin{aligned}
\Psi(k) & =\sum_{i=k}^{i=k+N}\left(\|u(i)\|_{Q}^{2}+\|z(i)\|_{R}^{2}\right) \\
& =U(k)^{\prime} \mathcal{Q} U(k)+Z(K)^{\prime} \mathcal{R} Z(k)
\end{aligned}
$$

where $U(k)=\left[\begin{array}{llll}u(k) & u(k+1) & \ldots & u(k+N)\end{array}\right]^{\prime}, Z(k)=\left[\begin{array}{llll}z(k) & z(k+1) & \ldots & z(k+N)\end{array}\right]^{\prime}$, $\mathcal{Q}=I_{N} \otimes Q, \mathcal{R}=I_{N} \otimes R, N$ the horizon length, $\otimes$ the Kronecker product and $I_{N}$ the identity matrix of size $N$.

The outputs $Z(k)$ are themselves functions of the inputs $U(k)$ and the disturbances $D(k)=$ $\left[\begin{array}{llll}d(k) & d(k+1) & \ldots & d(k+N)\end{array}\right]^{\prime}:$

$$
\begin{aligned}
& \underbrace{\left[\begin{array}{c}
z(k) \\
z(k+1) \\
\vdots \\
z(k+N)
\end{array}\right]}_{Z(k)}=\underbrace{\left[\begin{array}{c}
C \\
C A \\
\vdots \\
C A^{N}
\end{array}\right]}_{\Phi} x(k)+\underbrace{\left[\begin{array}{cccc}
0 & & & 0 \\
C B & 0 & & \\
\vdots & \ddots & \ddots & \\
C A^{N-1} B & \cdots & C B & 0
\end{array}\right]}_{\Gamma} \underbrace{\left[\begin{array}{c}
u(k) \\
u(k+1) \\
\vdots \\
u(k+N)
\end{array}\right]}_{U(k)} \\
& +\underbrace{\left[\begin{array}{cccc}
0 & & & 0 \\
C G & 0 & & \\
\vdots & \ddots & \ddots & \\
C A^{N-1} G & \cdots & C G & 0
\end{array}\right]}_{\Gamma_{d}} \underbrace{\left[\begin{array}{c}
d(k) \\
d(k+1) \\
\vdots \\
d(k+N)
\end{array}\right]}_{D(k)} \\
& Z(k)=\Phi x(k)+\Gamma U(k)+\Gamma_{d} D(k)
\end{aligned}
$$

Combining 1.3 and $1.4 \mathrm{~b}$ leads to

$$
\Psi(k) \equiv U(k)^{\prime} \underbrace{\left[\mathcal{Q}+\Gamma^{\prime} \mathcal{R} \Gamma\right]}_{H} U(k)+\underbrace{\left[\Gamma^{\prime} \mathcal{R} \Phi x(k)+\Gamma^{\prime} \mathcal{R} \Gamma_{d} D(k)\right]^{\prime}}_{g} U(k)
$$




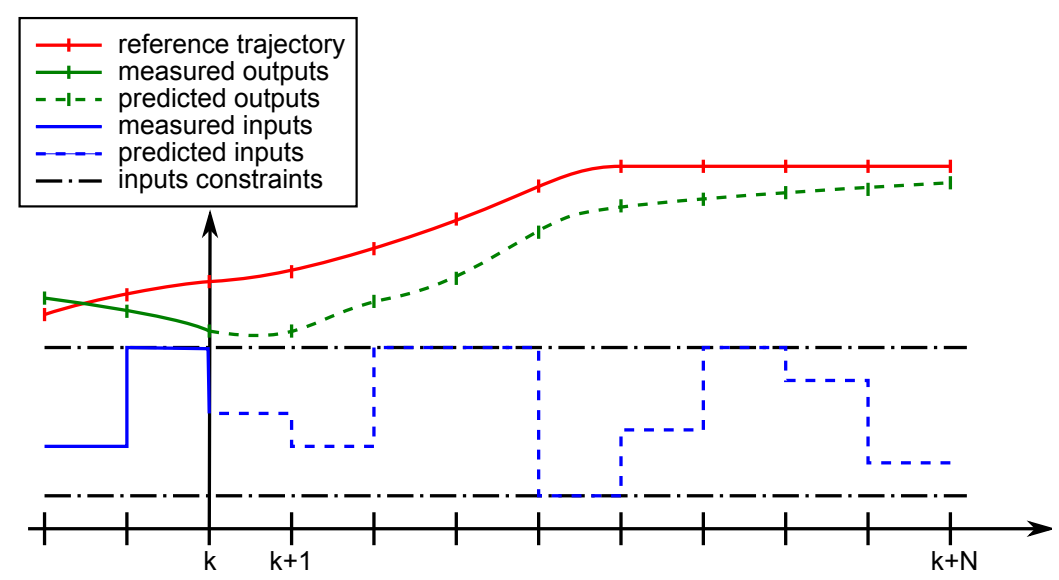

Figure 1.1: Model predictive control scheme: the predicted inputs are calculated in order to minimise a quadratic cost on, for example, the predicted inputs (dashed blue line) and the difference between the predicted outputs (dashed green line) and the reference trajectory (solid red line).

where the $\equiv$ sign denotes that the terms independent of $U(k)$ have beem omitted.

The MPC optimisation problem can be written as the quadratic program

$$
\begin{aligned}
\min _{U(k)} & \Psi(k)=\frac{1}{2} U(k)^{\prime} H U(k)+g^{\prime} U(k) \\
\text { s.t. } & u \in \mathbb{U}
\end{aligned}
$$

The quadratic program is solved at each time step $k$. The first element $u(k)$ is then applied to the system, and the MPC optimisation problem is run again at the next time step.

This basic structure can easily be adapted to take into account constraints on the outputs, to consider different horizon lengths for the costs on the inputs and on the outputs or to add a cost on the inputs increment as well. Some very efficient codes [5, 26, 3, 44, to solve the quadratic program $1.6 \mathrm{a}$ and $1.6 \mathrm{~b}$ already exist, which makes this formulation of model predictive control implementable on a real-time hardware. The work described in this thesis aims at implementing a model predictive control in a wind turbine. The frequency-weighted model predictive controls detailed further in the thesis keep the same structure of the quadratic program.

The advantages of model predictive control over other control theories are its ability to operate closer to limits and to handle constraints. The performance of all smart rotor concepts is restricted by constraints on the actuators; for example in the case of trailing edge flaps, limits on the rate at which the flaps can be moved and maximum flap deflection in each direction. The presence of these constraints makes model predictive control a suitable candidate.

Model predictive control has been applied to wind energy. Henriksen et al. [34, 35, 33, designed a wind turbine model predictive controller and a non linear model predictive control for a floating wind turbine. Thomsen et al. 54 worked on robust stability in model predictive control. Evans et al. 25] is also working on robust model predictive control of wind turbines. 


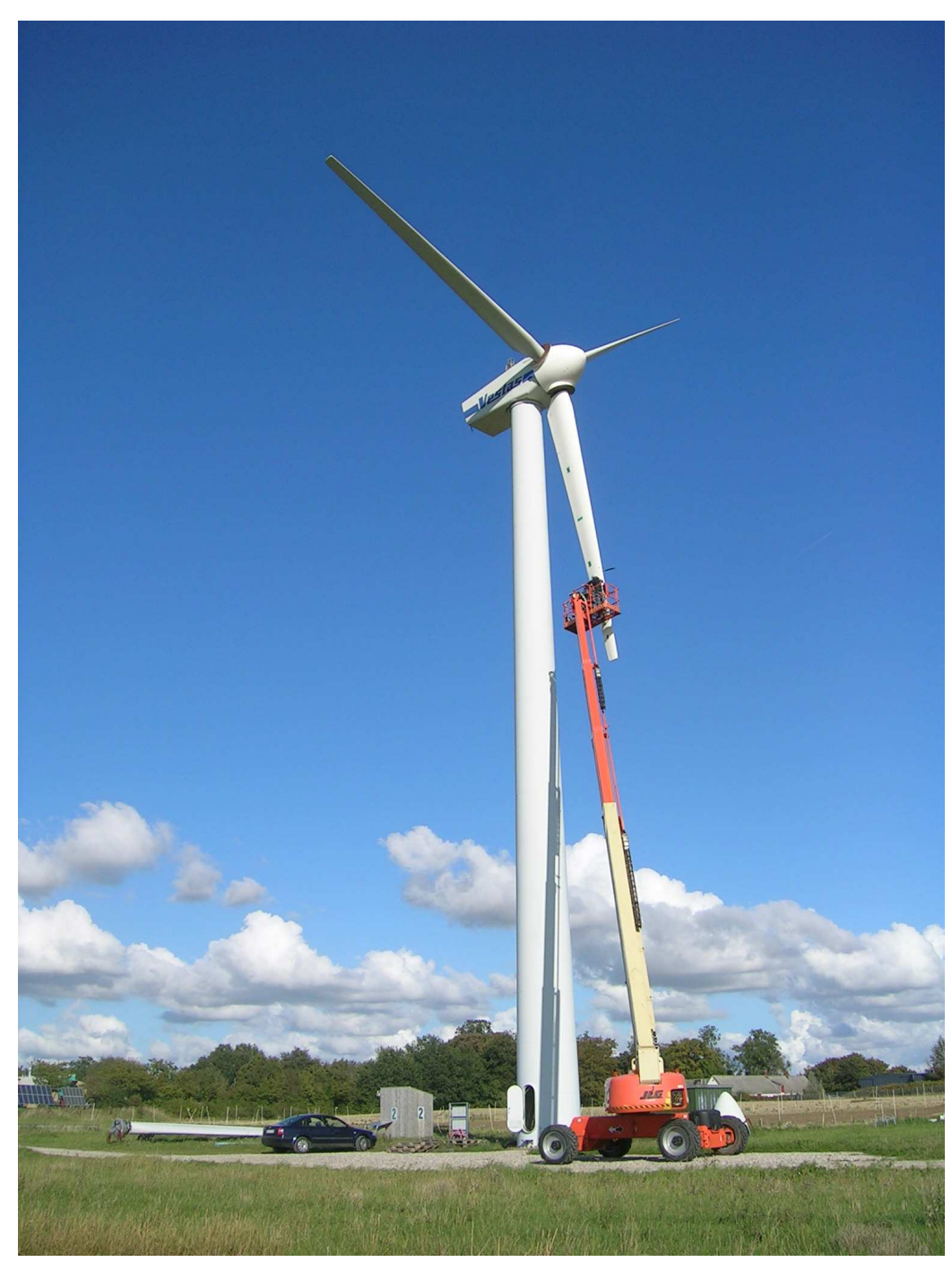

Figure 1.2: Picture of the V27 demonstrator turbine. The cherry picker is used to access the hatches in the blade when servicing the actuators and the sensors.

\subsection{ATEF project and V27 demonstrator}

This thesis is part of the Adaptive Trailing Edge Flap (ATEF) project launched in 2008, in a collaboration between the Risø DTU, DTU MEK and Vestas Wind Systems A/S [1. One of the work packages consisted in proving load alleviation on the Vestas V27 wind turbine located at the Ris $\varnothing$ campus of the Technical University of Denmark (Figure 1.2).

The V27 turbine is an horizontal axis wind turbine, with a nominal power of $225 \mathrm{~kW}$, and a rotor diameter of $27 \mathrm{~m}$. It operates at two constant rotor speeds, $32 \mathrm{rpm}$ at wind speeds lower than 4 to $5 \mathrm{~m} . \mathrm{s}^{-1}$ and $43 \mathrm{rpm}$ at higher wind speeds. The collective pitch of the turbine is used for regulating the power production only. Designed in the eighties, the Vestas V27 is a rather stiff turbine, compared to modern turbines. 


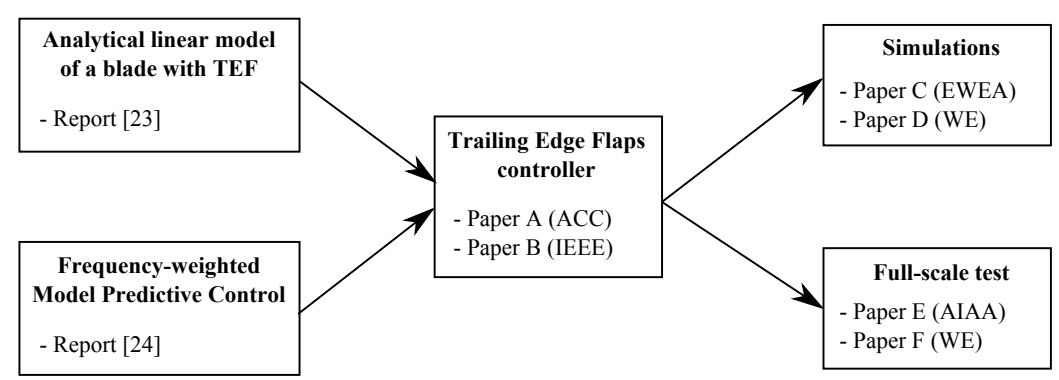

Figure 1.3: Research areas covered in this thesis

\subsection{Contributions}

The "smart" rotor concept gathers research in various areas like sensors, actuators, control theory, $2 \mathrm{D}$ and $3 \mathrm{D}$ aerodynamics, wind turbine modeling, simulation models, wind tunnel tests etc. The research presented in this thesis is driven by the full-scale test on the V27 wind turbine with trailing edge flaps, and by the constraints related to the experiment in terms of sensors, actuators and hardware available. Figure 1.3 summarises the research areas covered in this thesis.

Model Predictive Control was chosen for its ability to handle constraints. The trailing edge flaps on the test turbine are expected to operate up to their maximum deflections because they are too small to alleviate fully the flapwise blade root loads. The technical report "Frequency-weighted model predictive control" 24] describes three approaches to perform frequency-weighted model predictive control, by introducing filters, zero-phase filters or discrete Fourier transform in the cost function.

The design model for the trailing edge flap controller is an analytical linear model of a spinning blade with trailing edge flaps. Both blade root strain gages and leading edge Pitot tubes, present on the demonstrator blade, are part of this design model. The model is described in the technical report "Analytical linear model of a blade with trailing edge flaps (for design of model based controls)" 23].

Papers $\mathrm{A}$ and $\mathrm{B}$ describe the trailing edge flaps controller, the Kalman filter used to estimate the model states and the wind speed estimator necessary to perform gain scheduling of the the controller. Paper A describes how to use the frequency-weighted model predictive control in order to target at loads with specific frequencies and to avoid unnecessary high frequency actuation of the trailing edge flaps. In paper $B$, the three approaches of frequency-weighted model predictive control are studied, highlighting the benefits and the drawbacks of each method based on Flex5 simulations of the V27 turbine. Results from the full-scale test are also presented.

Papers $\mathrm{C}$ and $\mathrm{D}$ present results from simulations. In paper $\mathrm{C}$, the results from simulations of the trailing edge flaps controller on a multi-megawatt wind turbine are presented. Only normal production load cases are considered. Fatigue and extreme load reductions, as well as power loss and trailing edge flap activity are investigated. Paper $\mathrm{D}$ focuses on robustness studies regarding the simulation models: the same trailing edge flaps controller, with the same tuning, is used in both Flex5 and HAWC2 simulations with different model complexities.

Papers $\mathrm{E}$ and $\mathrm{F}$ describe the results from the full-scale test. In paper E only open-loop controls were performed. In paper $\mathrm{F}$, fatigue load reduction achieved on the test turbine is presented. A $14 \%$ flapwise blade root fatigue load reduction was achieved in a 38 minute test. This papers also shows some comparisons with Flex5 simulations. 


\subsection{Outline of the thesis}

Chapters 2, 3 and 4 summarise the main conclusions of the papers included in this thesis, and include some details and comments which could not be inserted in the published or submitted papers.

Chapter 2 is dedicated to the trailing edge flap controller design, including the wind speed estimator, the Kalman filter, the design model and the frequency-weighted model preditive control. It is based on papers $\mathrm{A}$ and $\mathrm{B}$ and on the technical reports [23] and [24].

Chapter 3 summarises the results of the simulations. It is based on papers $\mathrm{A}, \mathrm{C}$ and $\mathrm{D}$.

Chapter 4 is dedicated to the results from the full-scale test on the Vestas V27 wind turbine (papers $\mathrm{E}$ and F . Both open loop and closed loop tests were run. 
Chapter 2

\section{Control system design}

This chapter summarises papers $\mathrm{A}$ and $\mathrm{B}$ and technical reports [23] and 24]. Some further explanations on the wind turbine loads, the wind speed estimator and the disturbance model are given in this chapter.

The trailing edge flaps controller design is restricted by the experimental setup of the demonstrator turbine, regarding the available sensors, actuators and controller hardware. In particular, the pitch controller of the demonstrator turbine could not be modified, and the trailing edge flaps controller runs independently of the wind turbine controller (Figure 2.1). This setup has the advantage of being relatively easy to implement on the demonstrator turbine. Such a setup is however not optimal, and the trailing edge flaps controller has to be designed in order to avoid any interaction between the two controllers which could lead to instabilities. On the V27, the pitch controller performs power control only, pitching out of the wind when the produced power is higher than the turbine nominal power. The pitch is thus actuated at frequencies below the $1 \mathrm{P}$ frequency. On the other hand, the trailing edge flap controller performs loads control only, and acts at frequencies higher than or equal to the $1 \mathrm{P}$ frequency.

The trailing edge flaps controller loop includes a wind speed estimator, a predictive Kalman filter and a model predictive control of the trailing edge flaps. Those three components of the controller are detailed in this chapter. A low-level controller, provided by the actuator manufacturer, ensures that the actuators track the reference command.

\subsection{Wind turbine loads}

The trailing edge flaps controller main objective is to reduce the flapwise blade root fatigue loads. Understanding the origin of those fatigue loads is necessary to design an efficient trailing edge flaps controller.

The flapwise blade root fatigue loads are dominated by the aerodynamic loads. Figure 2.2 shows that the dominant relative contribution to the flapwise blade root fatigue damage of the NREL off- 


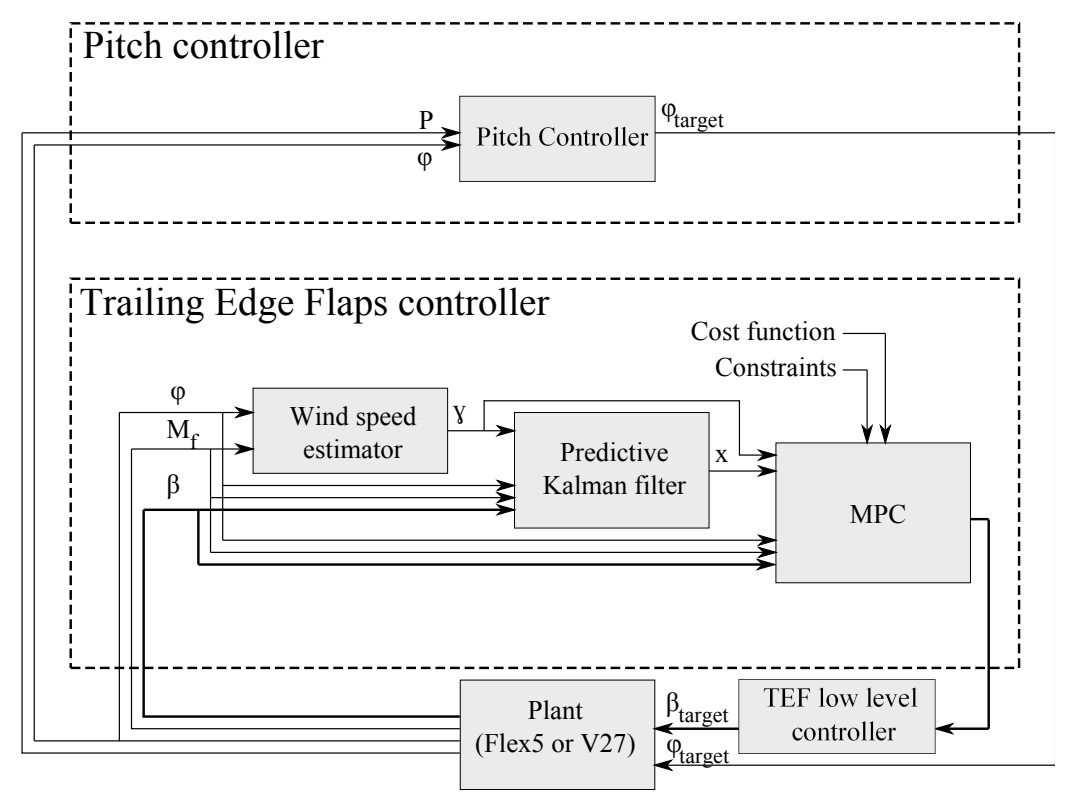

Figure 2.1: Illustration of the pitch controller and the trailing edge flaps controller of the V27 wind turbine. $P$ is the produced power, $\varphi$ the pitch angle, $M_{f}$ the flapwise blade root moment of the blade with trailing edge flaps, $\beta$ the trailing edge flap angles, $\gamma$ the estimated wind speed and $\mathbf{x}$ the design model states.

shore $5 \mathrm{MW}$ reference wind turbine 37 occurs above rated power, at the $1 \mathrm{P}, 2 \mathrm{P}$ and $3 \mathrm{P}$ frequencies [16.

A non turbulent wind which hits the rotor with a yaw or tilt angle angle w.r.t. to the rotor plane, or with a non-null wind shear, creates loads which are rotor azimuth dependent (Figure 2.3). Most of the energy of those loads is at the $1 \mathrm{P}$ frequency, corresponding to one event per rotor revolution.

Tower shadow, which reduces the wind speed around the tower, creates a more sudden change in the inflow seen by the rotating blade, both in terms of wind speed and inflow angle. It results in the excitation of the wind turbine eigenmodes and thus transfers energy at the frequencies of the wind turbine eigenmodes. The tower shadow excites mainly the blade first eigenfrequencies.

Most of the energy of the wind turbulence is contained in the low frequencies, below $0.5 \mathrm{~Hz}$ (Figure 2.4). However, wind turbulence does not only generate time dependent wind speeds and directions, but also induces wind speeds and directions which depend on the rotor azimuth angle. A rotating blade goes then through areas with azimuth dependent wind speeds and directions. As a consequence, the wind speed seen by a rotating blade, at a given radius, also shows a strong $1 \mathrm{P}$ to $3 \mathrm{P}$ content. This is called rotational sampling.

As a conclusion, even if the free wind speed is highly stochastic, the wind speed seen by a rotating blade has a high content at the $1 \mathrm{P}$ frequency and at higher harmonics $(2 \mathrm{P}$ and $3 \mathrm{P})$. This makes it possible then to get a rough estimate of the predicted wind speeds seen by the rotating blade, which improves significantly the performance of the trailing edge flaps controller. 


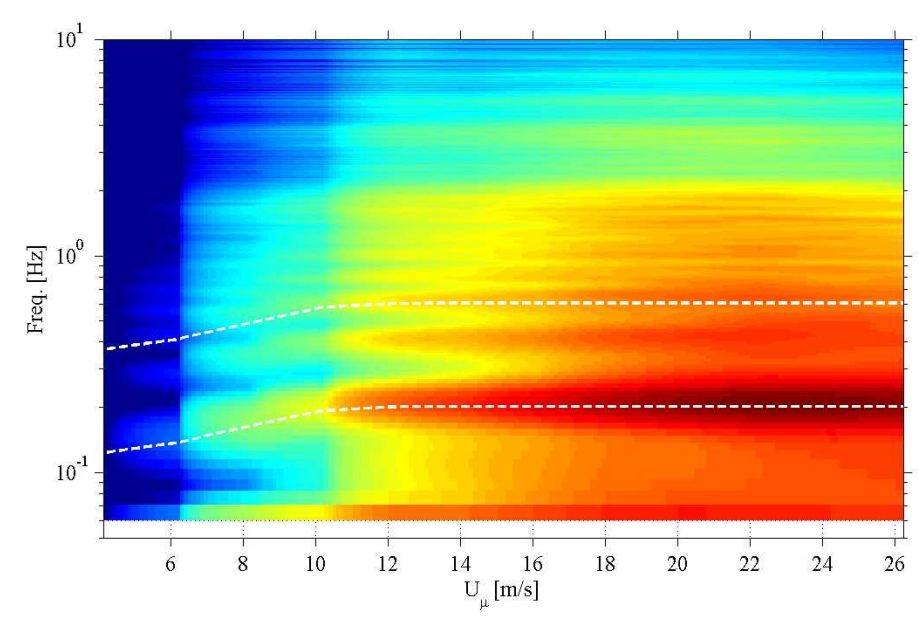

Figure 2.2: Flapwise blade root bending moment, relative contributions to fatigue damage ratio, as function of frequency and mean wind speed bin. Dashed white lines mark the $1 \mathrm{P}$ and $3 \mathrm{P}$ frequencies, red colored patches identify higher fatigue contributions. From Bergami [16]

\subsection{Wind speed estimator and gain scheduling}

The blade model, and especially the DC gain from trailing edge flaps to flapwise blade root moment, varies as a function of the mean free wind speed. The higher the wind speed, the higher the DC gain from trailing edge flap angle to flapwise blade root moment (Figure 2.5). Gain scheduling on the mean free wind speed is thus required to ensure performance of the trailing edge flaps controller over the whole operating wind speeds range (from around 4 to $20 \mathrm{~m} / \mathrm{s}$ during the full-scale test). The mean free wind speed, which is similar to a time average over a few rotor rotations and a spatial average over the rotor area, can not be measured directly with the available sensors. Østergaard et al. developed a method to estimate accurately the effective wind speed of a wind turbine [46]. Such a method can not be used on the V27 because of its fixed rotor speed. The mean free wind speed is instead estimated roughly from the pitch position and the flapwise blade root moment: below rated power, the pitch position is close to 0 , and the flapwise blade root moment increases as a function of the mean free wind speed. Above rated power, the pitch position increases and the flapwise blade root moment decreases as a function of the mean free wind speed (Figure 2.6. A low-pass filter ensures that the estimated free wind speed is a smooth function of time.

Such a simplified method is not very accurate, especially around rated power, but has the advantage of being fast to compute, and of requiring only the flapwise blade root moment and the pitch position. These two sensors are among the available sensors to the trailing edge flap controller. A small error on the estimated mean free wind speed is however not critical as it is only used for gain scheduling and not for power control.

Gain scheduling of the matrices for both the Kalman filter and the Model Predictive Control is based on this estimated mean free wind speed $\gamma$, by linear interpolating the Kalman filter and the MPC matrices. 

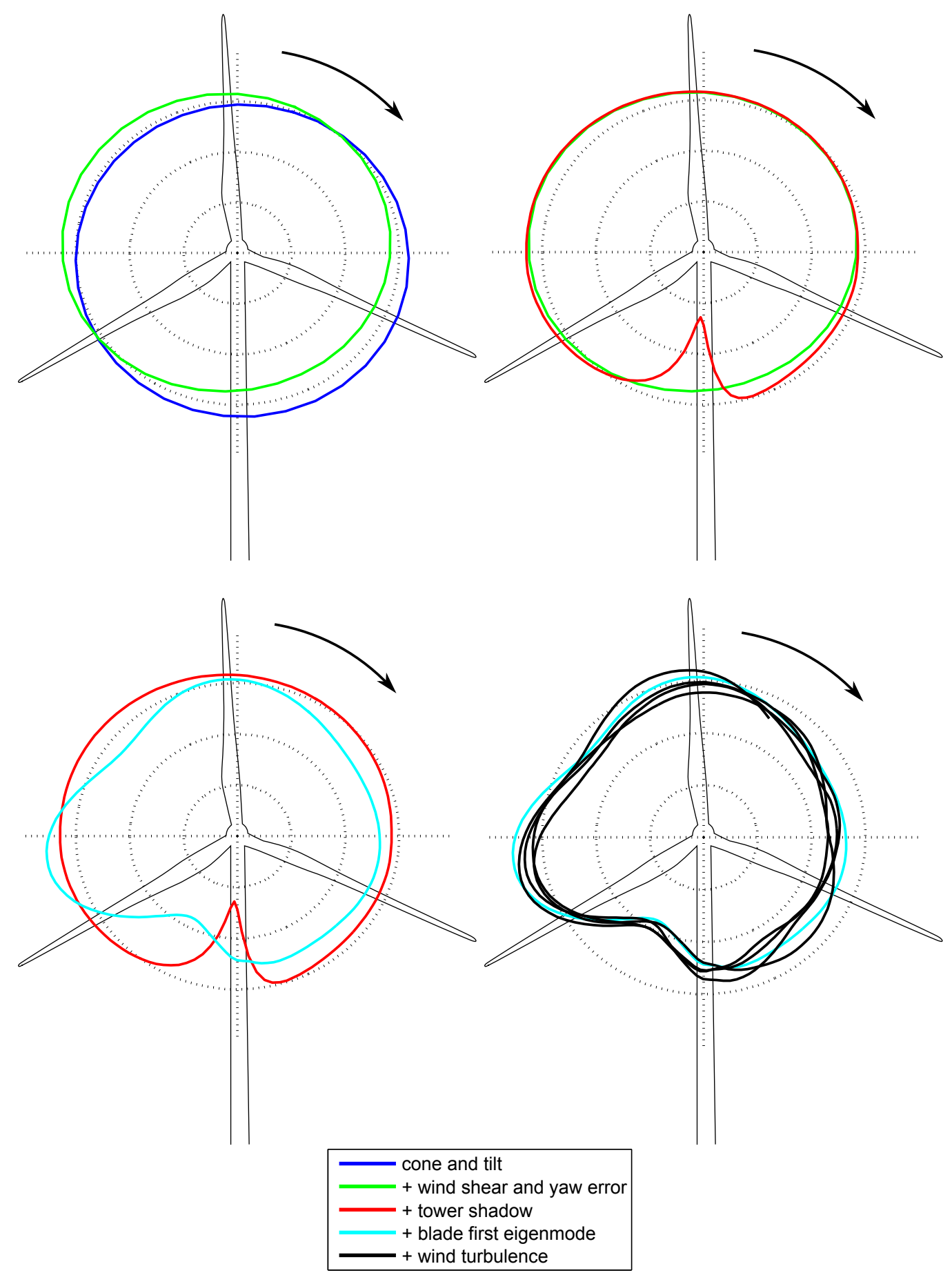

Figure 2.3: Illustration of the flapwise blade root bending moment on a stiff wind turbine with a non-null cone and tilt angle (blue). Wind shear and a yaw error are then added to the inflow (green). Taking into account the tower shadow creates sudden changes in the flapwise blade root moment when the blade passes the tower (red) and excites the first blade eigenmode (light blue). At last, on top of this inflow is added the wind turbulence (black). 


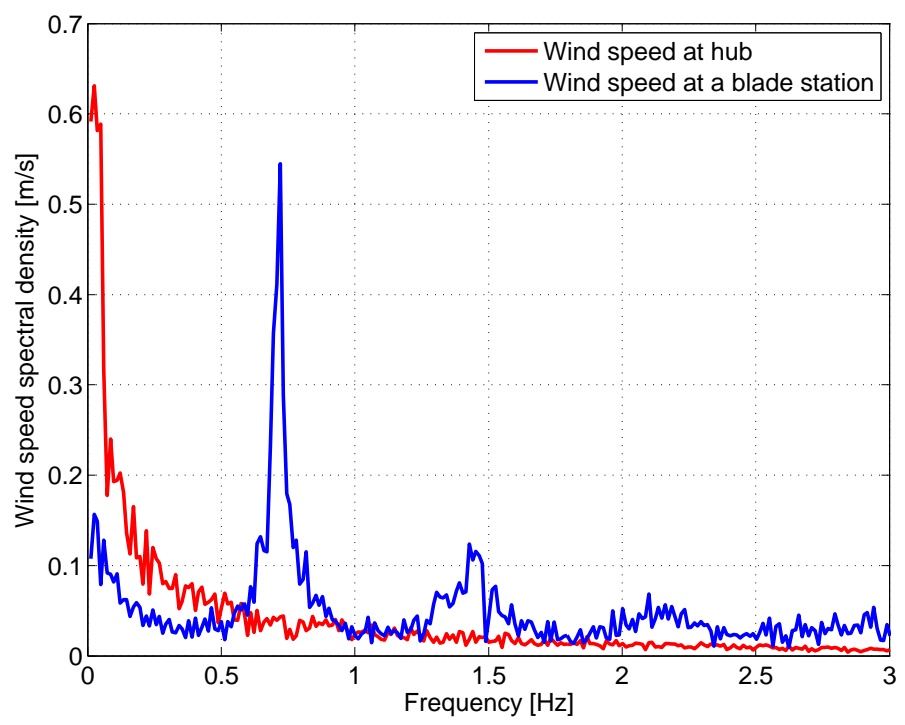

Figure 2.4: Spectral density of the wind speed simulated at the turbine hub (red) and at a given radius of a rotating blade (blue). The rotor rotates at around $43 \mathrm{rpm}$, the $1 \mathrm{P}$ frequency is around $0.7 \mathrm{~Hz}$. The 1P and higher harmonics present in the spectral density of the wind speed seen by the rotating blade are due to the rotational sampling of the turbulent wind.

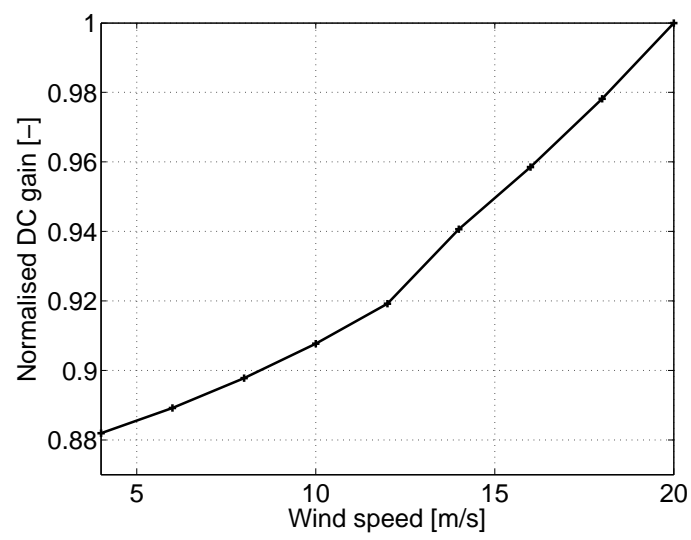

Figure 2.5: Normalised DC gain, from trailing edge flap angle to flapwise blade root bending moment for a rotating blade, at different mean free wind speeds. 


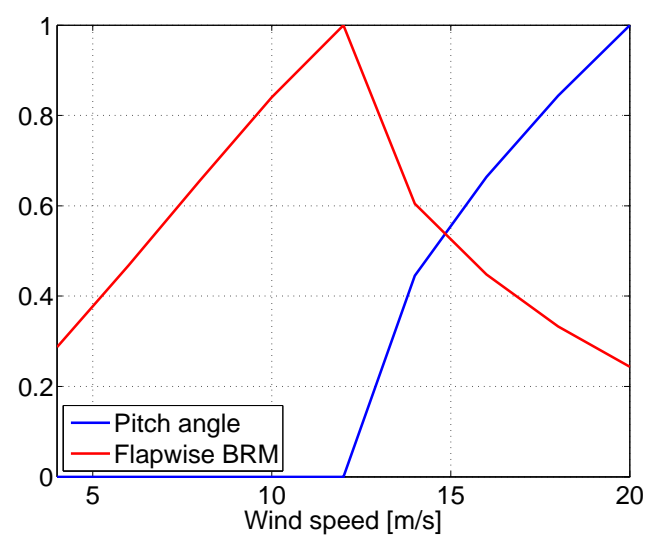

Figure 2.6: Illustration of the pitch angle and flapwise blade root moment (BRM) of the V27 at different mean free wind speeds. Data are normalised.

\subsection{Model Predictive Control}

\subsubsection{Design model}

The performance of model-based controls depends highly on the accuracy of the design model of the controlled system.

Andersen et al. 9] showed that the trailing edge flaps controller should react fast to the mesured disturbances in order to keep a high efficiency of the trailing edge flaps. A 50 ms delay would almost half the fatigue load reduction compared to the case with no delays. Such a result, even if it's obtained with a simple proportional controller on the high pass filtered flapwise blade root moment, and without retuning the controller to take into account the delay, indicates that delays should be kept to a minimum. The V27 high rotor speed corresponds to around 30 degrees rotation per $100 \mathrm{~ms}$. Wind conditions may vary a lot 30 degrees apart of each other, which confirms that delays have to be kept small. It was thus decided to run the trailing edge flaps controller at $50 \mathrm{~Hz}$ at least, corresponding to a 5.2 degree rotation of the rotor. Non-linear model predictive control, which is very CPU consuming, was thus not an option. Linear design models, derived from system identification or from first principle models, are then required.

Van der Veen et al. developed and tested on a scaled turbine with trailing edge flaps a method to perform closed-loop system identification of wind turbines [57, 36. This specific method is pertinent for identifying the design model of the rotating blade with trailing edge flaps as it is designed to identify the model without capturing the strong periodic components in the outputs due to wind shear, gravity or tower shadow for example. However, system identification is a time consuming task which requires several hours of measurements at a given wind condition. During the system identification, the trailing edge flaps are actuated at frequencies which cover at least the frequencies of interest for the controller, i.e. up to the first blade eigenfrequency. Such a high activity of the actuators could have damaged the prototype trailing edge flaps. It was then decided to test system identification at the end of the test plan, but not to use it to derive the design models.

Accurate linearised models of the whole wind turbine can also be generated from aero-servo-elastic tools like HAWCStab2 [31] for example. However, such models are very large, several hundreds 
states, and are not suited for controller design.

Instead, an analytical linear model of a rotating blade with trailing edge flaps was developed from first principle models. The structural model of the blade is based on the modal approach, where the blade dynamics is modeled by a linear combination of the dynamics of its first eigenmodes 32. The aerodynamics of the induced velocity and of the $2 \mathrm{D}$ airfoil lift and drag coefficients are neglected in the design model because their time constants are respectively significantly larger and smaller than the time constants of interest for the trailing edge flaps controller, i.e. the $1 \mathrm{P}$ to $4 \mathrm{P}$ frequencies.

In spite of those simplifications, the Bode plots, from trailing edge flap angle to flapwise blade root moment, of the analytical linear model and of the Flex5 models match very well. The Flex5 model of the V27 turbine was derived from an older Flex4 model (See Section 3.1.2). Figure 2.7 shows the Bode plots of the analytical linear model where the blade is modeled by two states only $\left(1^{s t}\right.$ blade eigenmode), and of the Flex5 models where the wind turbine is modeled as a stiff turbine, except for the first blade eigenmode, and as a flexible turbine as used to carry out simulations. In this figure, the turbine operates at its high rotor speed. The Flex5 non-linear simulations are run with a trailing edge flap stroke of $+/-5$ degrees, which is close to the maximum trailing edge flap deflection angle achievable on the demonstrator turbine. Differences in phase at high frequencies are due to the extra phase lags present in the 2D aerodynamics model of the airfoil [8], which are modeled in Flex5 but not in the analytical model. Differences between the Flex5 stiff and flexible models around the $1 \mathrm{P}$ and the $5 \mathrm{P}$ frequencies are the consequence of respectively the first tower and the second blade eigenmode.

The analytical linear blade model has the advantage of matching well the Flex5 model at the frequencies of interest between the $1 \mathrm{P}$ and the $4 \mathrm{P}$ frequencies, with only two states. However, this method requires an accurate structural (mass and stiffness distribution etc.) and aerodynamic model (airfoils lift and drag coefficients) of the real blade. This analytical design model does not adapt to the actual blade properties, and would thus be slightly off because of production geometrical tolerances or operating conditions like ice on the blades or erosion of the profiles. Such a problem does not happen with system identification when performed on-site. Figure 2.8 shows a comparison of the Bode plots of the analytical linear model and of the identified linear model, from trailing edge flap angle to flapwise blade root moment, for the V27 demonstrator turbine. This comparison clearly shows an important difference in the first blade eigenfrequency between the analytical model and the identified model. No accurate mass and stiffness distribution of the blade with trailing edge flaps were available. Their estimate lead to a difference in the blade flapwise eigenfrequency of around $10 \%$.

The analytical blade model, linearised at the steady point reached for a $\gamma$ mean free wind speed, is then:

$$
\begin{aligned}
\dot{\mathbf{x}}_{b} & =\mathbb{A}_{b}(\gamma) \mathbf{x}_{b}+\mathbb{B}_{b}(\gamma) \mathbf{u}+\mathbb{G}_{b}(\gamma) \mathbf{d}+\mathbf{w}_{b} \\
\mathbf{z} & =\mathbb{C}_{b} \mathbf{x}_{b} \\
\mathbf{y} & =\mathbb{C}_{b}^{m} \mathbf{x}_{b}+\mathbf{v}
\end{aligned}
$$

where $\mathbf{x}_{b}=\left[\begin{array}{ll}x_{1} & \dot{x}_{1}\end{array}\right]^{\prime}$ is the vector of the blade states. $x_{1}$ is the blade eigenmode generalised coordinate. $\mathbf{u}=[\beta]^{\prime}$ is the inputs vectors of the trailing edge flap angles. The measured disturbances $\mathbf{d}$ are either the pitch position only $\mathbf{d}=[\varphi]$ or the pitch angle and the Pitot tube measurements $\mathbf{V}_{P}: \mathbf{d}=\left[\begin{array}{ll}\varphi & \mathbf{V}_{P}\end{array}\right]^{\prime}$. The output $\mathbf{z}$ and the measurement $\mathbf{y}$ are the flapwise blade root bending moment. $\mathbf{w}_{b}$ is the process noise, and $\mathbf{v}$ is the measurement noise.

The trailing edge flaps actuators are assumed to be perfect. 

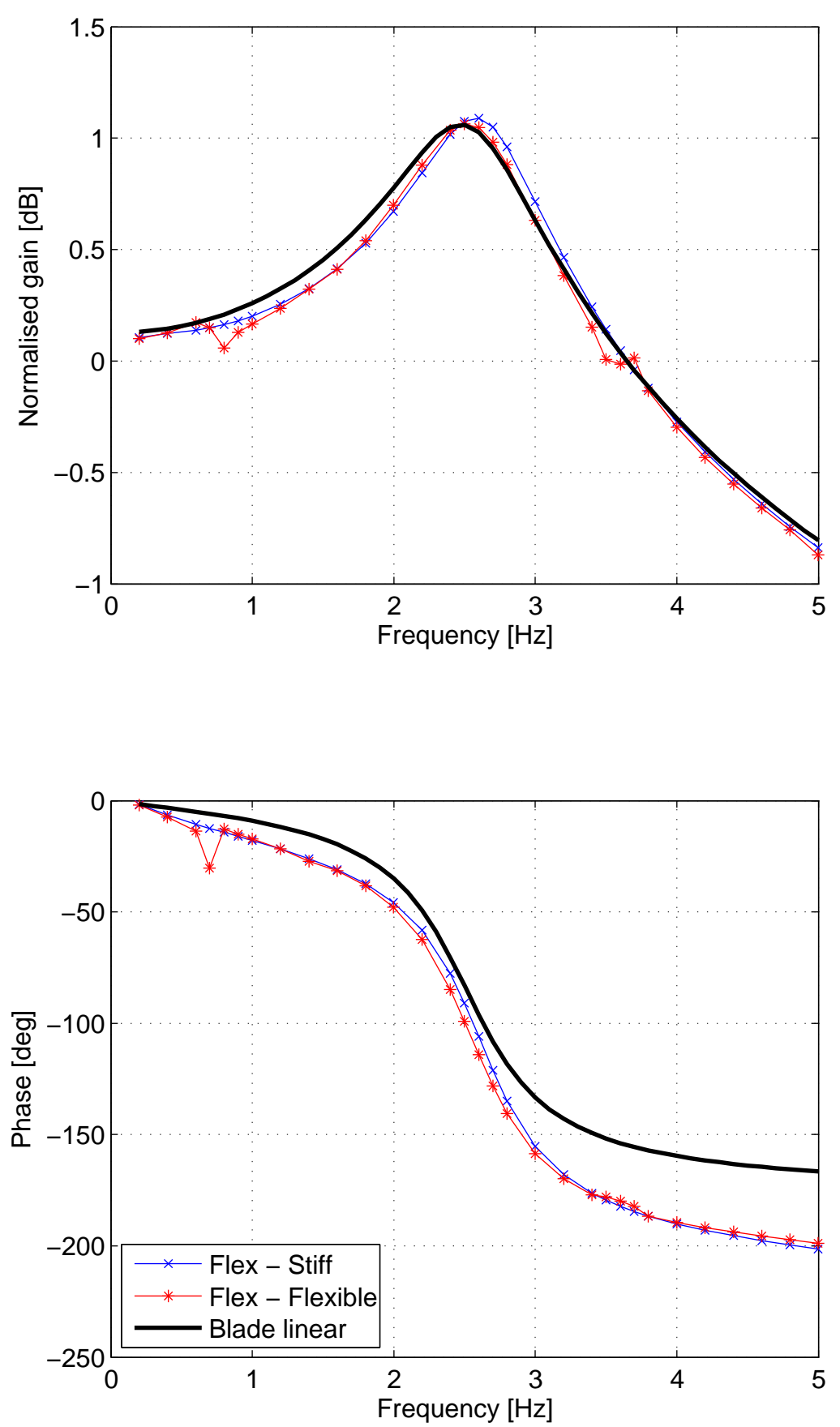

Figure 2.7: Bode plot for the SISO system trailing edge flap angle to flapwise blade root moment, when the V27 turbine operates at its high rotor speed $(43 \mathrm{rpm})$. The blue line refers to simulations run in Flex, with a stiff turbine where only the first blade eigenmode is flexible. The red line refers to simulations run in Flex with the model of a turbine where all the main turbine components are modeled as flexible structures. The thick black line refers to the analytical linear model as described in this thesis. 

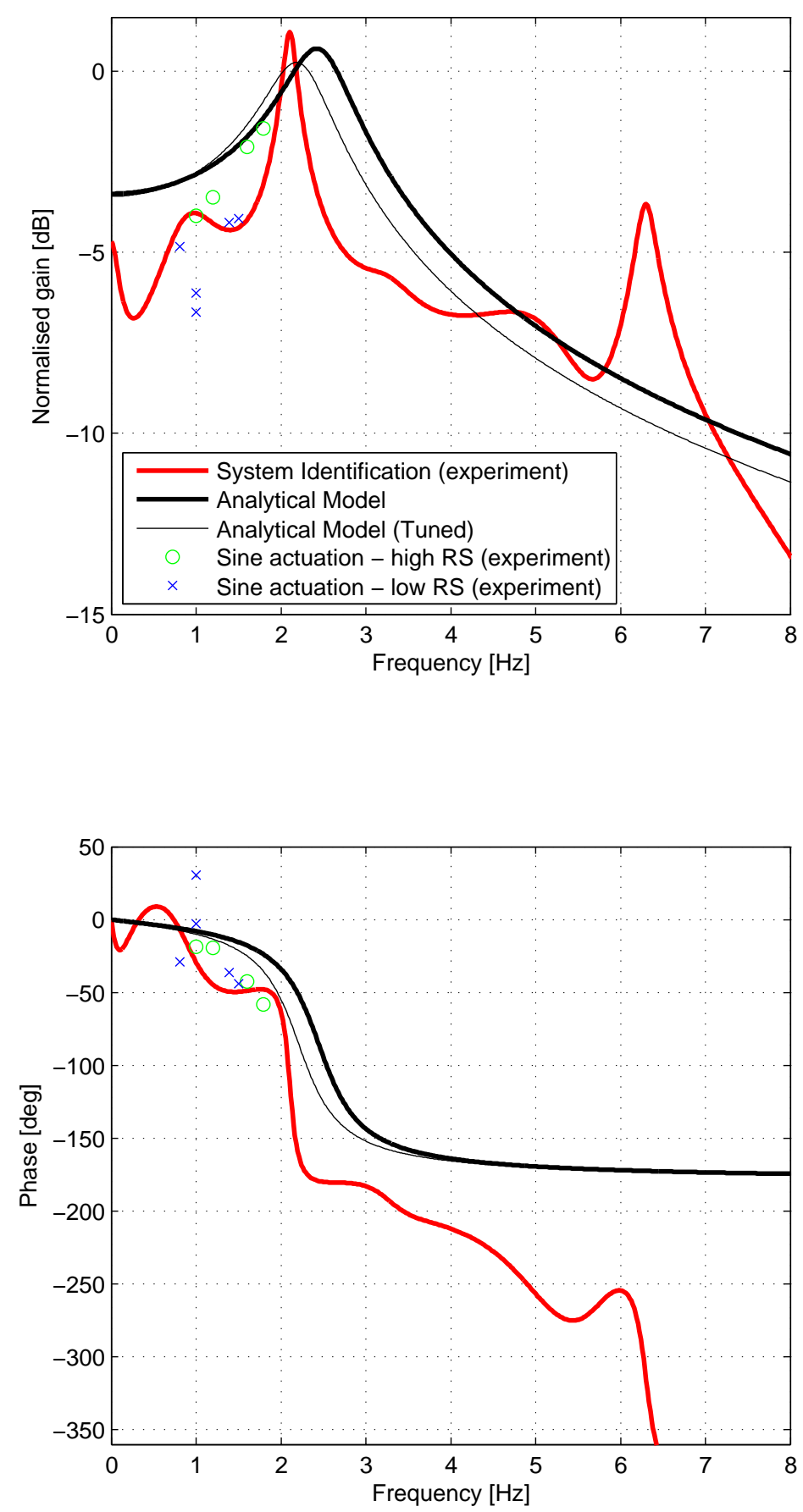

Figure 2.8: Bode plots from actual trailing edge flap angle position to flapwise blade root moment, for the identified model (thick red line), the analytical model used in the design of the model predictive control (thick black line), and for a tuned analytical model where the trailing edge flap efficiency has been reduced by $20 \%$ and the blade mass and stiffness distribution have been modified in order to decrease the blade first flapwise eigenfrequency (thin black line). The system identification was performed when the turbine was operating at its low rotor speed (32 rpm). The analytical models are derived at the same rotor speed. The responses from a sine actuation of the trailing edge flap, at different frequencies, at the high rotor speed (green circles) and at the low rotor speed (blue crosses) are also plotted. 
More details about the derivation of the analytical linear model and more comparisons with Flex5 simulations can be found in [23].

\subsubsection{Disturbance models}

The blade model previously described does not include any models of the disturbances creating the flapwise blade root loads. The disturbance model is thus a very important part of the controller design in order to improve the estimation of the blade states and the prediction of the model outputs. An accurate disturbance model improves significantly the performance of the trailing edge flaps controller.

As seen in Section 2.1 the highest contribution to the fatigue flapwise blade root loads comes from the $1 \mathrm{P}$ to $3 \mathrm{P}$ cyclic loads created by the external loads (wind shear, yaw error, tower shadow, wind turbulence etc.). Some periodic disturbance states $\zeta_{1 P}, \zeta_{2 P}, \zeta_{3 P}$, respectively at the $1 \mathrm{P}, 2 \mathrm{P}$ and $3 \mathrm{P}$ frequencies, are added to model the wind speed change as seen by a rotating blade. Those periodic disturbance states model the effect of wind shear, yaw error or even tower shadow and spatial wind turbulence on the rotating blade. The disturbances $\zeta_{1 P}, \zeta_{2 P}, \zeta_{3 P}$ are sine functions at the fixed $1 \mathrm{P}, 2 \mathrm{P}$ and $3 \mathrm{P}$ frequencies. Only the sine frequency is fixed in the disturbance model, the amplitude and the phase are estimated indirectly by the Kalman filter based on the measurements and on the blade model. The disturbances amplitude and phase then varies depending on the local wind conditions, on site.

$$
\underbrace{\left[\begin{array}{l}
\dot{\zeta}_{1 P} \\
\ddot{\zeta}_{1 P} \\
\dot{\zeta}_{2 P} \\
\ddot{\zeta}_{2 P} \\
\dot{\zeta}_{3 P} \\
\ddot{\zeta}_{3 P}
\end{array}\right]}_{\dot{\zeta}_{P}}=\underbrace{\left[\begin{array}{cccccc}
0 & 1 & 0 & 0 & 0 & 0 \\
-\omega_{1 P}^{2}(\gamma) & 0 & 0 & 0 & 0 & 0 \\
0 & 0 & 0 & 1 & 0 & 0 \\
0 & 0 & -\omega_{2 P}^{2}(\gamma) & 0 & 0 & 0 \\
0 & 0 & 0 & 0 & 0 & 1 \\
0 & 0 & 0 & 0 & -\omega_{3 P}^{2}(\gamma) & 0
\end{array}\right]}_{\mathbb{A}_{P}(\gamma)} \underbrace{\left[\begin{array}{c}
\zeta_{1 P} \\
\dot{\zeta}_{1 P} \\
\zeta_{2 P} \\
\dot{\zeta}_{2 P} \\
\zeta_{3 P} \\
\dot{\zeta}_{3 P}
\end{array}\right]}_{\zeta_{P}}+\underbrace{\left[\begin{array}{c}
w_{P_{1}} \\
w_{P_{2}} \\
w_{P_{3}} \\
w_{P_{4}} \\
w_{P_{5}} \\
w_{P_{6}}
\end{array}\right]}_{\mathbf{w}_{P}}
$$

where $\zeta_{P}$ is the vector of periodic disturbances, $\mathbf{w}_{P}$ is a vector of process white noise $\mathbf{w}_{P}\left(0, Q_{P}(\gamma)\right)$, and $\omega_{1 P}(\gamma), \omega_{2 P}(\gamma)$ and $\omega_{3 P}(\gamma)$ are respectively the $1 \mathrm{P}, 2 \mathrm{P}$ and $3 \mathrm{P}$ frequencies.

The periodic disturbances are assumed to act like a change in wind speed measured by a Pitot tube on the blade. Newton's second law applied to the first blade eigenmode is [23]:

$$
\begin{aligned}
M_{g}^{1} \ddot{x}_{1}+C_{g}^{1} \dot{x}_{1}+K_{g}^{1} \breve{x}_{1}= & \frac{\partial F_{g}(1)}{\partial x_{1}}(1) \breve{x}_{1}+\frac{\partial F_{g}(1)}{\partial \varphi} \breve{\varphi}+\sum_{F=1}^{3} \frac{\partial F_{g}(1)}{\partial \beta(F)}(F) \beta(F) \\
& +\sum_{P=1}^{3} \frac{\partial F_{g}(1)}{\partial V_{y 4}^{r e l}(I(P))}(P) \breve{V}_{y 4}^{r e l}(I(P)) \\
& +\sum_{P=1}^{3} \frac{\partial F_{g}(1)}{\partial V_{z 4}^{r e l}(I(P))}(P) \breve{V}_{z 4}^{r e l}(I(P))
\end{aligned}
$$

The periodic disturbances are assumed to model a change in the wind speed measured by the Pitot tubes, in the direction of the mean free wind speed $\left(z_{4}\right.$ in 23$\left.]\right)$. The governing equation 2.3 
becomes then

$$
\begin{aligned}
M_{g}^{1} \ddot{x}_{1}+C_{g}^{1} \dot{x}_{1}+K_{g}^{1} \breve{x}_{1}=\frac{\partial F_{g}(1)}{\partial x_{1}}(1) \breve{x}_{1}+\frac{\partial F_{g}(1)}{\partial \varphi} \breve{\varphi}+\sum_{F=1}^{3} \frac{\partial F_{g}(1)}{\partial \beta(F)}(F) \beta(F) \\
+\sum_{P=1}^{3} \frac{\partial F_{g}(1)}{\partial V_{y 4}^{r e l}(I(P))}(P) \breve{V}_{y 4}^{r e l}(I(P)) \\
+\sum_{P=1}^{3} \frac{\partial F_{g}(1)}{\partial V_{z 4}^{r e l}(I(P))}(P)\left(\breve{V}_{z 4}^{r e l}(I(P))-\zeta_{1 P}-\zeta_{2 P}-\zeta_{3 P}\right) \\
+\underbrace{\sum_{P=1}^{3} \frac{\partial F_{g}(1)}{\partial V_{z 4}^{r e l}(I(P))}(P)}_{A_{\zeta}}\left(\zeta_{1 P}+\zeta_{2 P}+\zeta_{3 P}\right)
\end{aligned}
$$

Another input disturbance state $\zeta_{s s}$ is added to the model in order to cope with the low frequencies plant/model mismatch. It is modeled as the integral of a white noise.

$$
\dot{\zeta}_{s s}=\zeta_{s s}+w_{s s}
$$

where $w_{s s}$ is a process white noise $w_{s s}\left(0, Q_{s s}(\gamma)\right)$.

If the first blade eigenmode and the $1 \mathrm{P}, 2 \mathrm{P}$ and $3 \mathrm{P}$ periodic disturbances are considered, the controller design model becomes:

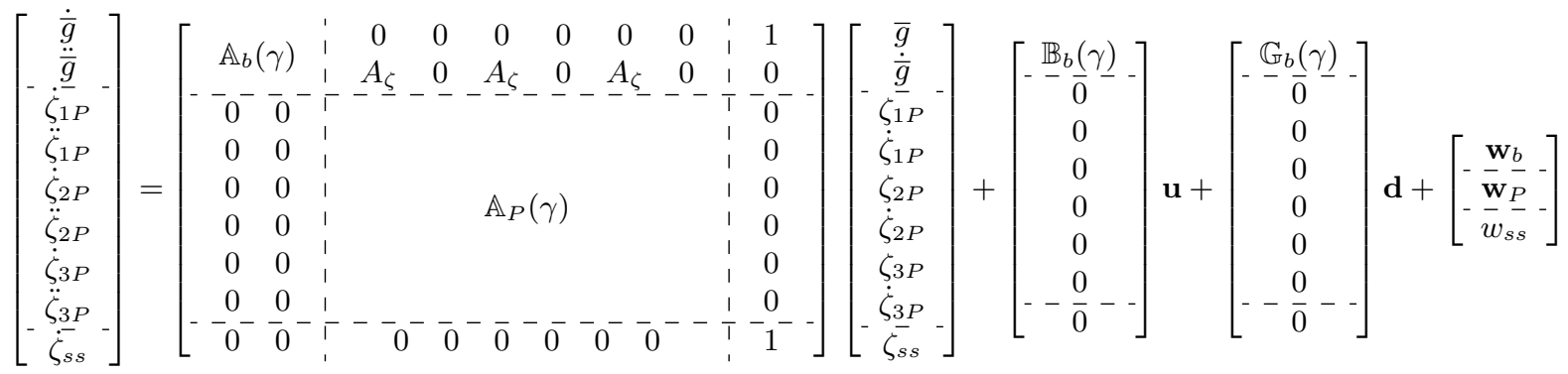

$$
\begin{aligned}
& \mathbf{z}=\mathbf{y}=\left[\begin{array}{llllllll:l}
\mathbb{C}_{b} & 0 & 0 & 0 & 0 & 0 & 0 & 0
\end{array}\right]\left[\begin{array}{c}
\bar{g} \\
\dot{\bar{g}} \\
\hdashline \zeta_{1 P} \\
\dot{\zeta}_{1 P} \\
\zeta_{2 P} \\
\dot{\zeta}_{2 P} \\
\zeta_{3 P} \\
\dot{\zeta}_{3 P} \\
\hdashline \zeta_{s s}^{-}
\end{array}\right]+\mathbf{v}
\end{aligned}
$$

Note that if Pitot tube measurements are used with this disturbance model, the measurable dis- 
turbances vector $\mathbf{d}$ becomes

$$
\mathbf{d}=\left[\begin{array}{c}
\breve{\varphi} \\
\breve{V}_{y 4}^{r e l}(I(1)) \\
\breve{V}_{y 4}^{r e l}(I(2)) \\
\breve{V}_{y 4}^{r e l}(I(3)) \\
\breve{V}_{z 4}^{r e l}(I(1))-\zeta_{1 P}-\zeta_{2 P}-\zeta_{3 P} \\
\breve{V}_{z 4}^{r e l}(I(2))-\zeta_{1 P}-\zeta_{2 P}-\zeta_{3 P} \\
\breve{V}_{z 4}^{r e l}(I(3))-\zeta_{1 P}-\zeta_{2 P}-\zeta_{3 P}
\end{array}\right]
$$

\subsubsection{Kalman filter}

The discretised controller design model is now noted:

$$
\begin{aligned}
x_{k+1} & =A x_{k}+B u_{k}+G d_{k}+w_{k} \\
y_{k} & =C^{m} x_{k}+v_{k}
\end{aligned}
$$

where $w_{k}$ is the process white noise, $w_{k}(0, Q(\gamma))$, and $v_{k}$ is the observation noise, $v_{k}(0, R(\gamma))$. $w_{k}$ and $v_{k}$ are assumed to be non correlated zero-mean Gaussian white noise.

At each time step $k+1$, a steady-state predictive Kalman filter estimates the states at time $k+1$ $\hat{x}_{k+1 \mid k}$ based on the measurements $y_{k}$, the inputs $u_{k}$, the measurable disturbances $d_{k}$ and the estimated states $\hat{x}_{k \mid k-1}$ at time step $k$.

$$
\hat{x}_{k+1 \mid k}=\left(A(\gamma)-K(\gamma) C^{m}\right) \hat{x}_{k \mid k-1}+B(\gamma) u_{k}+G(\gamma) d_{k}+K(\gamma) y_{k}
$$

where $K(\gamma)$ is the Kalman gain, and is calculated offline.

Because this filter is not adaptive, it is necessary to estimate the covariance $Q(\gamma)$ and $R(\gamma)$ offline, before running the simulations or the tests. The tuning of those covariances is not a trivial task and a poor tuning may lead to high loads increase in the turbine (Paper A). The tuning is made partly on a trial and error basis.

A first estimate of the variance $\mathbf{w}_{b}$ of the blade model is obtained by running Flex5 simulations and comparing the blade eigenmode generalised coordinates as simulated in Flex5 and estimated by the Kalman filter.

The variance $\mathbf{w}_{P}$ and $w_{s s}$ of the disturbances are tuned so that they actually capture the disturbances they are modeled for: the periodic disturbances should capture only the disturbances at the frequency they are designed for, and the quasi-steady disturbance should capture only the very slow disturbances. A high variance $\mathbf{w}_{P}$ allows the amplitude and the phase of the periodic disturbances to vary substantially. If the variance is too high, the disturbance may capture some events at higher frequencies than required and the disturbance model is no longer accurate. If the variance is too low, the amplitude of the phase of the periodic disturbances can no longer vary fast enough to capture change in local wind conditions. For example, it may not be fast enough to capture a change of wind shear from positive shear to negative shear. The disturbance state may then be out of phase, modeling a positive shear while the actual shear is negative, and the trailing edge flap controller may then act out of phase, resulting in loads increase. By the same token, a too high variance $w_{s s}$ would result in the quasi-steady disturbance capturing high frequency disturbances like the 1P disturbance with the wrong model.

The tuning of the estimator is crucial for the performance of the trailing edge flap controller. In particular, the tuning of the variance of the disturbances depend on the local wind conditions and 
different values should be used depending on the wind turbulence intensity for example. An adaptive estimator which would adapt better to the local wind conditions would improve substantially the performance of the controller.

\subsubsection{Frequency-weighted model predictive control}

The trailing edge flaps controller main objective is to reduce the flapwise blade root fatigue loads. The $1 \mathrm{P}$ loads have the largest contribution to those fatigue loads, followed by the $2 \mathrm{P}$ and the $3 \mathrm{P}$ loads, and the loads at the first blade eigenfrequency. The following requirements apply thus to the controller:

- the trailing edge flaps should not target loads at frequencies lower than the $1 \mathrm{P}$ frequency. It is important that, at each rotor rotation, the full trailing edge flaps amplitude is available in order to target the $1 \mathrm{P}$ loads. If the flaps were used to reduce the low frequency loads, the ability of the flaps to alleviate the $1 \mathrm{P}$ loads would be reduced, and the flapwise blade root fatigue load reduction would be decreased as well. Tuning the trailing edge flap controller not to target the low frequency loads also reduces the risk of interaction with the pitch controller.

- the trailing edge flaps should not target loads at frequencies higher than the first blade eigenfrequency. Such loads do not create much fatigue damage of the blade, and using the flaps to target them would unnecessarily wear the actuators. The design model is moreover not accurate enough at such frequencies.

Three different approaches of frequency-weighted model predictive control are tested. They are all based on conventional model predictive which require the solution of

$$
\begin{array}{ll}
\min _{\hat{U}} & \Psi(k)=\frac{1}{2} \hat{U}^{\prime} H \hat{U}+g^{\prime} \hat{U} \\
\text { s.t. } & U_{\min } \leq \hat{U} \leq U_{\max }
\end{array}
$$

where $\hat{U}$ is the vector of predicted inputs within the horizon, $U_{\min }$ and $U_{\max }$ are constraints on the inputs, and $\Psi(k)$ is a quadratic cost function. Efficient codes [5, 26, 3, 44] exist to solve such a nominal quadratic program, which makes the implementation of this model predictive control in a wind turbine possible. The three approaches consist then in deriving the different $H$ and $g$ matrices of the cost function.

\section{Method A:}

The first natural approach consists in writing a model predictive control cost function based on the filtered predicted inputs and outputs. Lowpass, highpass, bandpass or bandstop filters are designed in order to emphasise the frequencies of the inputs and outputs which are targeted. The trailing edge flaps actuation at frequencies below the $1 \mathrm{P}$ frequency and above the $3 \mathrm{P}$ frequency or the first blade eigenfrequency should have a higher cost than the actuation at frequencies between the $1 \mathrm{P}$ frequency and the $3 \mathrm{P}$ frequency. The model predictive control cost function consists then in a cost on the bandstop filtered inputs; the bandstop filter is tuned to decrease the inputs amplitude at the $1 \mathrm{P}$ to $3 \mathrm{P}$ frequencies. For the same reasons, a cost on the bandpass filtered flapwise blade root moment is added in order to focus the controller on the frequencies of interest for reducing the flapwise blade root fatigue loads.

Figure 2.9 illustrates this frequency-weigthed model predictive control. In this example, the cost on the predicted filtered flapwise blade root moment only depends on the $1 \mathrm{P}$ content of the predicted 
flapwise blade root moment. On the other hand, the cost on the predicted filtered trailing edge flap angle depends mainly on the high frequency content of the predicted inputs.

The cost function $\Psi(k)$ is the sum of a quadratic cost on the difference between the filtered predicted outputs $\tilde{z}(k+i \mid k)$ and the set points $r(k+i \mid k)$ and a quadratic cost on the filtered predicted inputs $\tilde{u}(k+i \mid k)$.

$$
\begin{aligned}
\Psi(k) & =\sum_{i=1}^{N} \frac{1}{2}\|\tilde{z}(k+i \mid k)-r(k+i \mid k)\|_{Q_{\tilde{z}}}^{2}+\sum_{i=1}^{N} \frac{1}{2}\|\tilde{u}(k+i \mid k)\|_{Q_{\tilde{u}}}^{2} \\
& \equiv \frac{1}{2} \hat{U}^{\prime}\left(H_{\tilde{z}}(\gamma)+H_{\tilde{u}}\right) \hat{U}+g(\gamma)^{\prime} \hat{U}
\end{aligned}
$$

with

$$
g(\gamma)=M_{\tilde{z}, R}(\gamma) R+M_{\tilde{z}, D}(\gamma) \hat{D}+M_{\tilde{z}, x_{1}}(\gamma) \hat{x}(k+1 \mid k)+M_{\tilde{z}, x_{\tilde{z}}}(\gamma) x_{z}(k+1 \mid k)+M_{\tilde{u}, x_{\tilde{u}}} x_{u}(k+1 \mid k)
$$

where $\hat{U}$ is the vector of predicted inputs $\hat{u}(k+i \mid k)$ within the MPC horizon, $R$ is the vector of set points, $\hat{D}$ is the vector of predicted measurable disturbances $\hat{d}(k+i \mid k), \hat{x}(k+1 \mid k)$ is the vector of the estimated design model states, $x_{z}(k+1 \mid k)$ and $x_{u}(k+1 \mid k)$ are the states of respectively the output and input filters. $H_{\tilde{z}}(\gamma), H_{\tilde{u}}, M_{\tilde{z}, R}(\gamma), M_{\tilde{z}, D}(\gamma), M_{\tilde{z}, x_{1}}(\gamma), M_{\tilde{z}, x_{\tilde{z}}}(\gamma), M_{\tilde{u}, x_{\tilde{u}}}$ are the Model Predictive Control matrices which are calculated offline. The sign $\equiv$ denotes that the terms independent of $\hat{U}$ have been removed from the equation as they are irrelevant when solving the quadratic program (2.10a and (2.10b). $N$ is the horizon length of the model predictive control.

\section{Method B:}

Another approach, similar to the previous one, consists in using zero-phase filters instead of conventional filters which add time lags in the system and reduce the performance of the frequencyweighted model predictive control. Figure 2.9 shows an important time lag on the filtered flapwise blade root moment which will lead to a lagged response from the trailing edge flaps, and thus a loss of efficiency of the controlled flaps. Such lags do not appear when zero-phase filters are used. The cost function $\Psi(k)$ is the sum of a quadratic cost on the difference between the zero-phase filtered predicted outputs $\tilde{z}(k+i \mid k)$ and the set points $r(k+i \mid k)$ and a quadratic cost on the filtered predicted inputs $\tilde{u}(k+i \mid k)$ :

$$
\begin{aligned}
\Psi(k) & =\sum_{i=1}^{N} \frac{1}{2}\|\overleftrightarrow{z}(k+i \mid k)-r(k+i \mid k)\|_{Q \overleftrightarrow{z}}^{2}+\sum_{i=1}^{N} \frac{1}{2}\|\overleftrightarrow{u}(k+i \mid k)\|_{Q \overleftrightarrow{u}}^{2} \\
& \equiv \frac{1}{2} \hat{U}^{\prime}\left(H_{\overleftrightarrow{Z}}(\gamma)+H_{\overleftrightarrow{U}}\right) \hat{U}+g(\gamma)^{\prime} \hat{U}
\end{aligned}
$$

with

$$
g(\gamma)=M_{\overleftrightarrow{z}, Z_{m}}(\gamma) Z_{m}+M_{\overleftrightarrow{z}, x_{1}}(\gamma) x_{1}+M_{\overleftrightarrow{z}, D}(\gamma) \hat{D}+M_{\overleftrightarrow{z}, R}(\gamma) R+M_{\overleftrightarrow{u}, U_{m}} U_{m}
$$

where $Z_{m}$ and $U_{m}$ are respectively the vector of measured outputs and inputs, and $H \overleftrightarrow{Z}(\gamma), H \overleftrightarrow{U}$, $M \overleftrightarrow{z}, Z_{m}(\gamma), M \overleftrightarrow{z}, x_{1}(\gamma), M \overleftrightarrow{z}, D(\gamma), M \overleftrightarrow{z}, R(\gamma)$, and $M \overleftrightarrow{u}, U_{m}$ are the Model Predictive Control matrices calculated offline. The design of model predictive control with costs on zero-phase filtered inputs and outputs is more complicated than the design with cost on filtered inputs and outputs. However, the model predictive control with costs on the zero-phase filtered inputs and outputs give substantially better results than when conventional filters are used. Moreover, those two approaches require similar horizon length. The quadratic programs solved at each time step have thus the same size, and the controllers run at approximately the same speed.

\section{Method C:}

At last, frequency-weighted model predictive control can be performed by adding costs in the 
frequency domain instead of the time domain. The Discrete Fourier Transform is performed on vectors including both measured data and predicted data, and the costs are added on the amplitude of the Discrete Fourier Transform coefficients of the inputs and outputs.

$$
\Psi(k)=\sum_{f=1}^{N_{f}} \frac{1}{2}\left\|X_{z}(f \mid k)\right\|_{Q_{X z}}^{2}+\sum_{f=1}^{N_{f}} \frac{1}{2}\left\|X_{u}(f \mid k)\right\|_{Q_{X_{u}}}^{2}
$$

where $X_{z}(f \mid k)$ and $X_{u}(f \mid k)$ are the amplitude of the Fourrier coefficients of respectively the flapwise blade root moment and the trailing edge flap angle, and $N_{f}$ is the number of frequencies available in the Discrete Fourier Transform.

$\Psi(k) \equiv \frac{1}{2} \hat{U}^{\prime}\left(\Re_{\mathcal{U}_{22}}+H_{\mathcal{Z}_{\mathrm{fft}}}(\gamma)\right) \hat{U}+\left[\Re_{\mathcal{U}_{12}}^{\prime} U_{m}+M_{\mathcal{Z}_{\mathrm{fft}}, x_{1}}(\gamma) \hat{x}(k+1 \mid k)+M_{\mathcal{Z}_{\mathrm{fft}}, \hat{D}}(\gamma) \hat{D}+M_{\mathcal{Z}_{\mathrm{fft}}, Z_{m}}(\gamma) Z_{m}\right]^{\prime} \hat{U}$

where $\Re_{\mathcal{U}_{22}}, H_{\mathcal{Z}_{\mathrm{fft}}}(\gamma), \Re_{\mathcal{U}_{12}}, M_{\mathcal{Z}_{\mathrm{fft}}, x_{1}}(\gamma), M_{\mathcal{Z}_{\mathrm{fft}}, \hat{D}}(\gamma)$ and $M_{\mathcal{Z}_{\mathrm{fft}}, Z_{m}}(\gamma)$ are the model predictive control matrices, calculated offline. This last approach has the advantage of giving a lot of flexibility in the frequencies to target. However, it requires longer horizons than the two previous approaches, which made this solution not suitable for the test on the demonstrator turbine.

More details about the three approaches can be found in paper B and in the technical report [24]. 

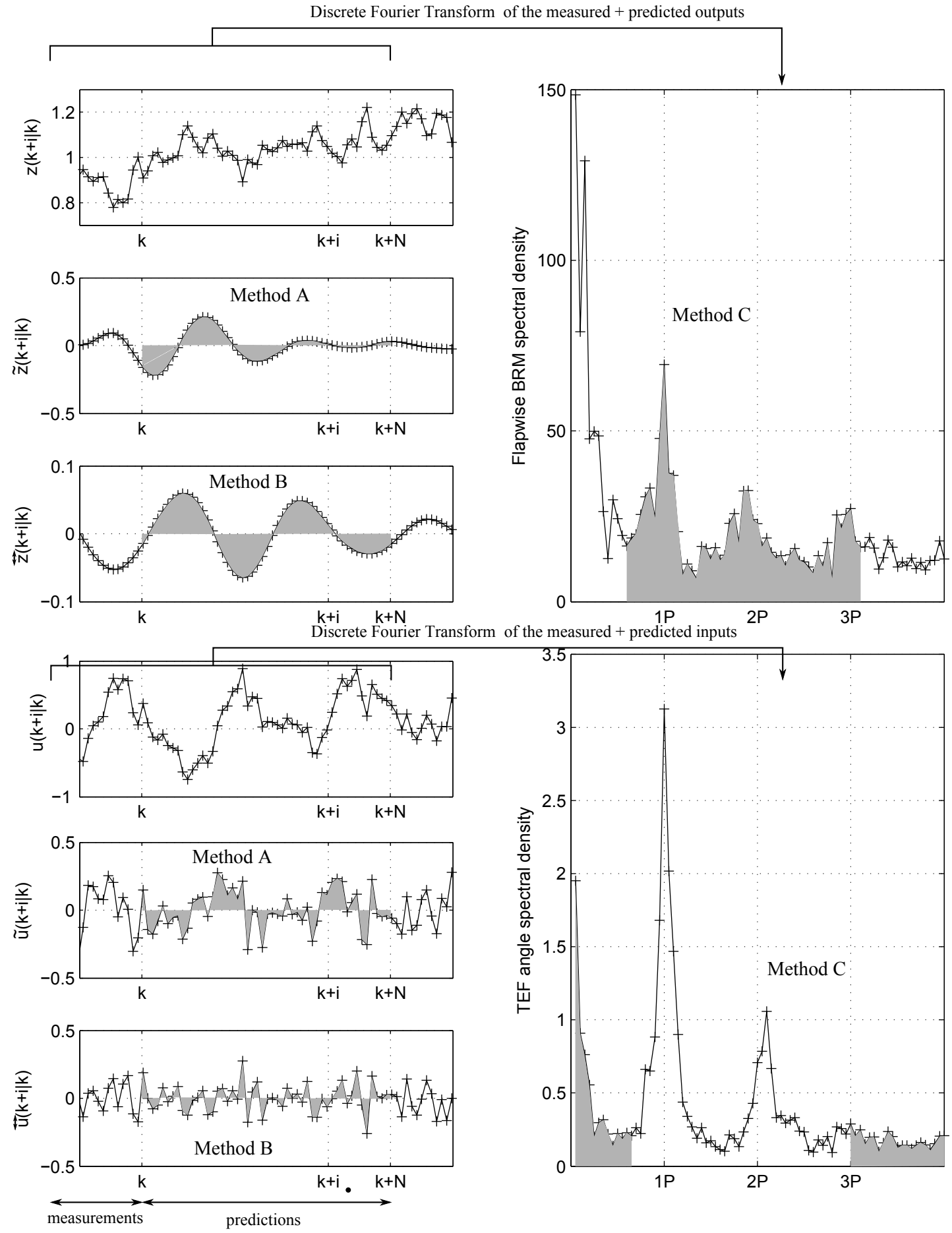

Figure 2.9: Illustration of the three approaches for performing frequency-weighted model predictive control. $z(k+i \mid k)$ is the output at time step $k+i$ predicted at time step $k$. The tilde denotes filtered values, and the over left-right arrow zero-phase filtered values. The same notation applies to the inputs $u(k+i \mid k)$. Grey areas represent the costs. 
Chapter 3

\section{Simulations}

This chapter summarises papers $\mathrm{C}, \mathrm{A}$ and $\mathrm{D}$.

\subsection{Simulation models}

\subsubsection{Aero-servo-elastic code for wind turbines with trailing edge flaps}

The aero-servo-elastic code used for simulation of wind turbine power production and loads is Flex5, developed at the department of Mechanical Engineering of the Technical University of Denmark DTU MEK 48. In Flex5, the tower, the shaft and the blades structure are parameterised by their first mode shapes. This modal approach is an efficient way to keep the number of the model states low, and thus to increase the simulation speed. The rotor aerodynamics is based on the Blade Element Momentum (BEM) theory with Glauert and Prandtl corrections [32, 21]. The original $2 \mathrm{D}$ blade section aerodynamics is based on the Øye dynamic stall model [47].

The ATEFlap model, an aerodynamics model of a deformable 2D airfoil, was implemented in Flex5 in order to simulate wind turbines with trailing edge flaps. The ATEFlap model combines Gaunaa's thin airfoil model 27] with the Beddoes Leishmann dynamic stall model described by Hansen et al. 29]. More information about the ATEFlap model can be found in Bergami and Gaunaa [17. Some minor modifications were made in order to make the code compatible with Flex5, and to meet the requirements a wind turbine manufacturer has. The code was modified to ensure that the quasi-steady values of lift, drag and moment coefficients given by the ATEFlap model match exactly the input polar files. This is important especially regarding power production.

A rough validation of the implementation of the ATEFlap model in Flex5 is made by comparing simulations run with the Øye dynamic stall model and with the ATEFlap model, with trailing edge flaps fixed in their neutral position (Figure 3.1). There is a good agreement in the amplitude of the 1P flapwise blade root loads. However, simulations with the ATEFlap model show smaller $2 \mathrm{P}$ and $3 \mathrm{P}$ loads. Time lags become more important at those frequencies, which highlights the differences 

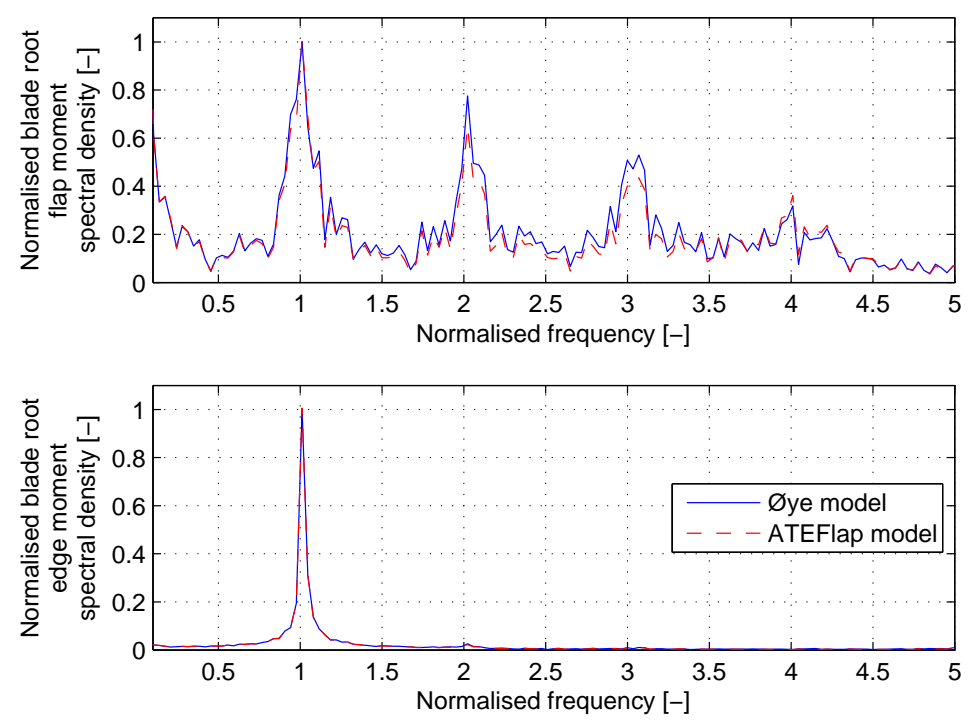

Figure 3.1: Normalised spectral densities of the flapwise and edgewise blade root moments, when simulations are run with the Øye dynamic stall model or the ATEFlap aerodynamics model.

between the two models. Fatigue and extreme loads of a wind turbine would thus depend on the aerodynamics model used. When calculating fatigue or extreme load reduction, it is important to run all simulations, with and without active trailing edge flaps, with the same model: the ATEFlap model.

No trailing edge flap actuator model is coded in Flex5. Perfect actuators are assumed.

\subsubsection{Flex5 model of the V27 wind turbine}

The Vestas V27 wind turbine was designed in the eighties, without using advanced aero-servoelastic tool like Flex5. An old Flex4 model of the V27 existed however. This model was not used for design of the turbine, but for some further studies. This Flex4 model was updated to fit Flex5 requirements. Technical documents about the specific demonstrator V27 turbine located at Risø DTU were used to adjust the model. Further tuning was performed after the first measurement campaign on the instrumented V27 turbine.

The V27 Flex5 simulations match fairly the measurements (Figure 3.2). It is however important to keep in mind that this model is still approximative, and does not have the accuracy that models of modern wind turbines have. In particular, the turbine controller, the blade profiles and the structural properties of the modified blade are thought to be points of the model where the level of tuning is uncertain.

Figure 3.2 shows a comparison of the flapwise and edgewise blade root moment spectral densities as simulated in Flex5/VTS and in HAWC2, and as measured on the demonstrator turbine. This comparison, based on 16 minute measurements, is not meant to be a validation of the model which would have required a longer measurement campaign and a better instrumented turbine and site. However, this comparison shows that the model is accurate enough to be used to get preliminary results. Differences between the measured and the simulated edgewise bending moment highlights 
a small difference in the frequency of the first blade edgewise eigenmode due to an error in the estimate of the mass and stiffness distribution of the modified blade. The first blade flapwise eigenmode is more excited in the simulations than in the measurements as well. This could be the consequence of an error in the modeling of either the wind turbine or the inflow conditions. This comparison was made with the first set of actuators tested on the demonstrator turbine.

\subsection{Trailing edge flap controller implementation}

The trailing edge flaps controller is coded in C++ and compiled into a Dynamic-Link Library (DLL). Coding in C++ allows the use of efficient libraries like the CBLAS library [2] for performing efficient algebra calculations and the qpOASES library [5, 26] for solving the quadratic program of the model predictive control. The C++ code also has the possibility to be compiled into a DLL for use in Flex5 simulations, and into a .out library for use the National Instruments real-time controller in the wind turbine. Almost the same code is then used in the simulations and on the turbine, which limits the risks of errors in the code running in the turbine; debugging a code is time consuming and could have been critical considering the short time available for the full-scale test. The implementation of the trailing edge flaps controller in Flex5 as a linked DLL also limits the risks of introducing errors in the Flex5 code.

A Runge-Kutta-Nyström integration scheme [32] is used in Flex5 to solve the second order differential equations resulting from the first principle models (Newton's second law). This integration scheme consists in four steps. At each time step $k$, the two first steps consist in calculating some estimations at time steps $k+\frac{1}{2}$, the third step is an estimation at time step $k+1$ and the last step gives the best estimates of the wind turbine states at time step $k+1$.

The trailing edge flaps controller is called only at the last step of the Runge-Kutta-Nyström integration scheme. The required measurements are then passed to the DLL, and the calculated predicted inputs $\hat{u}(k+1 \mid k)$ are passed from the DLL to Flex5. The predicted inputs $\hat{u}(k+1 \mid k)$ are however used in the Flex5 code only at the next time step in order to reproduce the experimental setup and the time necessary to compute the algorithm from measurements to trailing edge flap set point. At each time step, in Flex5, the trailing edge flaps controller is called following the call of the pitch controller.

\subsection{MPC performance, fatigue and extreme loads}

Comparing results from the litterature to results from this work is not trivial, because the authors run simulations with different simulations tools, wind turbine models, and load cases. Flapwise blade root fatigue load reduction documented in the litterature goes from 5 to $50 \%$. Because trailing edge flaps are meant for modern flexible turbines, the results presented in this section are not for the Vestas V27 turbine, but for a generic multi-megawatt turbine equipped with trailing edge flaps on its three blades. Simulations are run for the IEC 61400-1, ed.3 normal production load cases only (ntm turbulence only). The trailing edge flaps controller explicitely targets the flapwise blade root fatigue loads only. However, impact of the trailing edge flaps on the rest of the turbine is studied: both fatigue and extreme loads, in the blades, the main bearing and the tower are calculated.

Simulations show a significant fatigue and extreme load reduction in all the main wind turbine components (Table 3.1). Flapwise blade root fatigue loads, which are the only explicitely targeted 

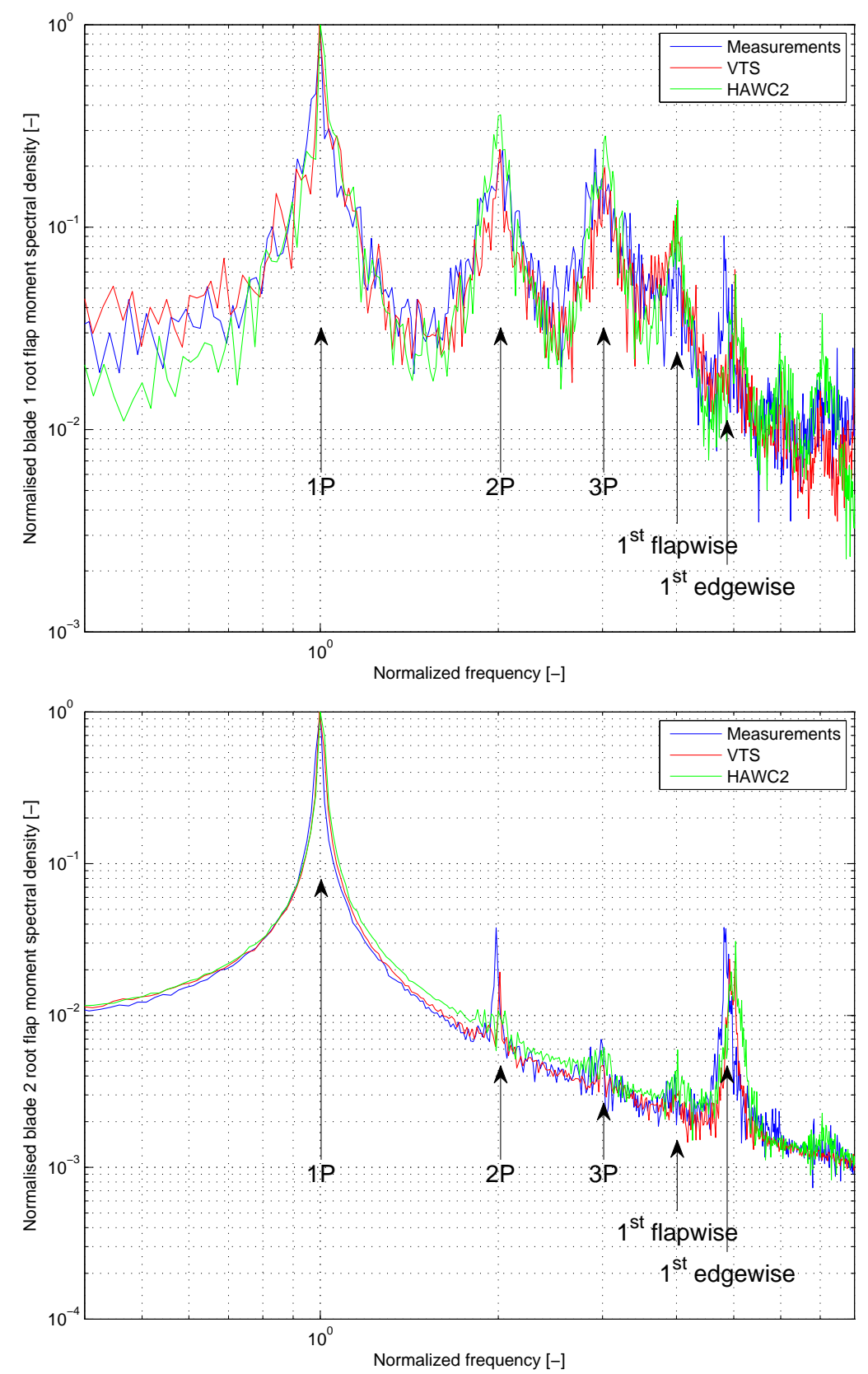

Figure 3.2: Comparison of the spectral densities of the simulated (Flex5/VTS and HAWC2) and the measured blade root bending moments for the V27 blade with trailing edge flaps when the TEF are fixed in their neutral position. Frequencies are normalized with respect to the 1P frequency. The power spectral densities are also normalized. 
Table 3.1: Fatigue and extreme load reduction achieved with trailing edge flaps. The Flex5 simulations are run on a generic multi-megawatt wind turbine.

\begin{tabular}{cc||c|c}
\multicolumn{2}{c}{ Sensor } & Fatigue [\%] & Extreme [\%] \\
\hline \multirow{4}{*}{ Blade } & Flapwise BRM & -22.8 & -6.8 \\
& Edgewise BRM & -3.0 & -4.0 \\
& Flapwise BTM & +2.1 & -2.8 \\
& Edgewise BTM & +6.9 & +6.8 \\
\hline \multirow{3}{*}{ Bearing } & Tilt moment & -32.1 & -11.0 \\
& Yaw moment & -32.2 & -4.7 \\
& Driving moment & +0.7 & +2.2 \\
\hline \multirow{3}{*}{ Tower } & Tilt top & -23.0 & -6.9 \\
& Yaw top & -24.4 & -10.2 \\
& Equivalent bottom & -11.7 & -17.5
\end{tabular}

by the trailing edge flaps controller, are reduced by $23 \%$. The main bearing and the tower also benefit largely from the trailing edge flaps: the main bearing tilt and yaw moments are reduced by $32 \%$, and the tower fatigue loads by 11 to $24 \%$. Extreme loads reductions should be considered with caution, as only normal production load cases were simulated. Extreme loads could happen during other load cases.

Those significant load reductions are however achieved at the cost of a power loss and a high activity of the trailing edge flaps. Power loss and trailing edge flap activity are two important factors when calculating the cost of energy. Such drawbacks of the use of trailing edge flaps should be improved.

The trailing edge flap controller was tuned in order to achieve a maximum flapwise blade root load reduction, without taking into account the power loss and the trailing edge flaps activity. A softer tuning of the trailing edge flaps controller would decrease the trailing edge flap activity and the power loss, without sacrificing much on the load reduction. The trailing edge flaps could also only be used above rated power, where there is no power loss, and where the flapwise blade root fatigue load reduction is the highest.

\subsection{Robustness}

The trailing edge flaps controller was designed in order to be tested on-site, on a real wind turbine. It is thus important to check the robustness of the controller before going on-site, and to make sure that no severe damage will happen to the wind turbine. The simulations presented in this section were run with the flexible generic multi-megawatt wind turbine.

The trailing edge flaps controller detailed in this report, and tested in simulations and on-site, is not designed explicitely to be robust. Robust model predictive control exists [25], but it could not be used real-time for this application because of the high CPU cost of robust model predictive control. Simulations on Flex5, with this trailing edge flaps controller, showed good results and no loads increase which would have damaged the turbine. However, the controller design model and the simulation model are very similar, both in terms of blade properties (mass, stiffness, twist distribution etc.) and in terms of governing equations (modal modeling of the blade structure). It is thus wise to run more simulations in order to check, at least partially, the robustness of the controller. 
Partial robustness regarding those two aspects is tested by running:

- Flex5 simulations with slightly different blade properties (mass distribution and trailing edge flap efficiency)

- HAWC2 simulations. HAWC2 [39] is another aero-servo-elastic code developed at the Technical University of Denmark, DTU Wind Energy. The structural model is based on a multibody finite element formulation [52. HAWC2, in particular, has the advantage of modeling the blade torsion degree of freedom, which is thought to be important when simulating blades with trailing edge flaps.

The Flex5 simulations show that the trailing edge flaps controller performance is not altered by a blade mass increase or decrease of 5\%. However, assuming a trailing edge flap efficiency $50 \%$ higher in the design model than in the simulation model leads to critical fatigue and extreme loads increase at the blade tip. There is a large uncertainty on the aerodynamic properties of the V27 airfoil with the trailing edge flap because no wind tunnel tests were performed. It is thus recommended to assume, in the controller design model, a lower than expected trailing edge flap efficiency.

The use of trailing edge flaps increase significantly the aerodynamic moment coefficient of the airfoils, leading to higher torsion and twist in the blade. The torsion degree of freedom of the blade is not part of the Flex 5 model, but is included in the HAWC2 finite element beam formulation. As expected, the introduction of the blade torsion degree of freedom leads to a lower flapwise fatigue load reduction, with a higher trailing edge flap activity. However, it did not yield to any instabilities. Modeling the blades with the non-linear multi-body formulation yields however to an unwanted actuation of the trailing edge flaps at high frequencies (between 7 and 12 times the 1P frequency). This would not happen with the real actuators as they would not be fast enough to be actuated at such high frequencies. However, tuning the trailing edge flaps controller less aggressive removed the unwanted excitation of the actuators at those high frequencies, with only small decrease of the loads reduction.

Such simulations do not guarantee that the trailing edge flaps controller will not be unstable on the demonstrator turbine. However, they indicate how to tune the controller in order to be more conservative and reduce the risk of instability. It also helps to understand how to tune the controller on-site if the controller does not act as expected. 
Chapter 4

\section{Experiments}

This chapter summarises papers $\mathrm{E}$ and $\mathrm{F}$.

\subsection{Setup}

The demonstrator turbine is the Vestas V27 located on Ris $\varnothing$ campus of the Technical University of Denmark in Roskilde, Denmark. This turbine has the advantage of being small and robust, and being located on the campus. It was thus relatively easy to access the turbine and especially the trailing edge flaps for maintenance and updates. The downside is that the turbine is smaller and stiffer than modern turbines, and is not a variable speed turbine. Figure 4.1 shows the installation of the rotor with the trailing edge flaps on the V27 wind turbine.

Three independent trailing edge flaps, each of them $100 \mathrm{~mm}$ wide and $700 \mathrm{~mm}$ long, are mounted on one of the three blades. A cabinet box with the data acquisition system, the real-time controllers and the input and output modules is fixed in the turbine spinner (Figure 4.2). Several watertight hatches are mounted on the blade in order to ease access to the actuators, the sensors, and the wires. Power is provided to the cabinet box and the actuators throught a slip ring mounted between the nacelle and the hub (Figure 4.3).

Two set of actuators were tested during the project. A first actuator system consisted in piezo electric benders excited by an actuation voltage varying from $-350 \mathrm{~V}$ to $900 \mathrm{~V}$. Those benders were protected by a rubber structure (Figure 4.4). Strain gages located on the piezo benders were used as position sensors. Those actuators had the advantage of being flexible, but they were not strong enough to compensate for the aerodynamic and the centrifugal loads under normal operation, and they were very fragile. Only a few open-loop tests with limited trailing edge flap deflection were run with those actuators.

The second set of actuators were hinged flaps actuated by a linear motor (Figure 4.5). Those actuators were able to achieve a much higher flap deflection, and were able to track well the set 


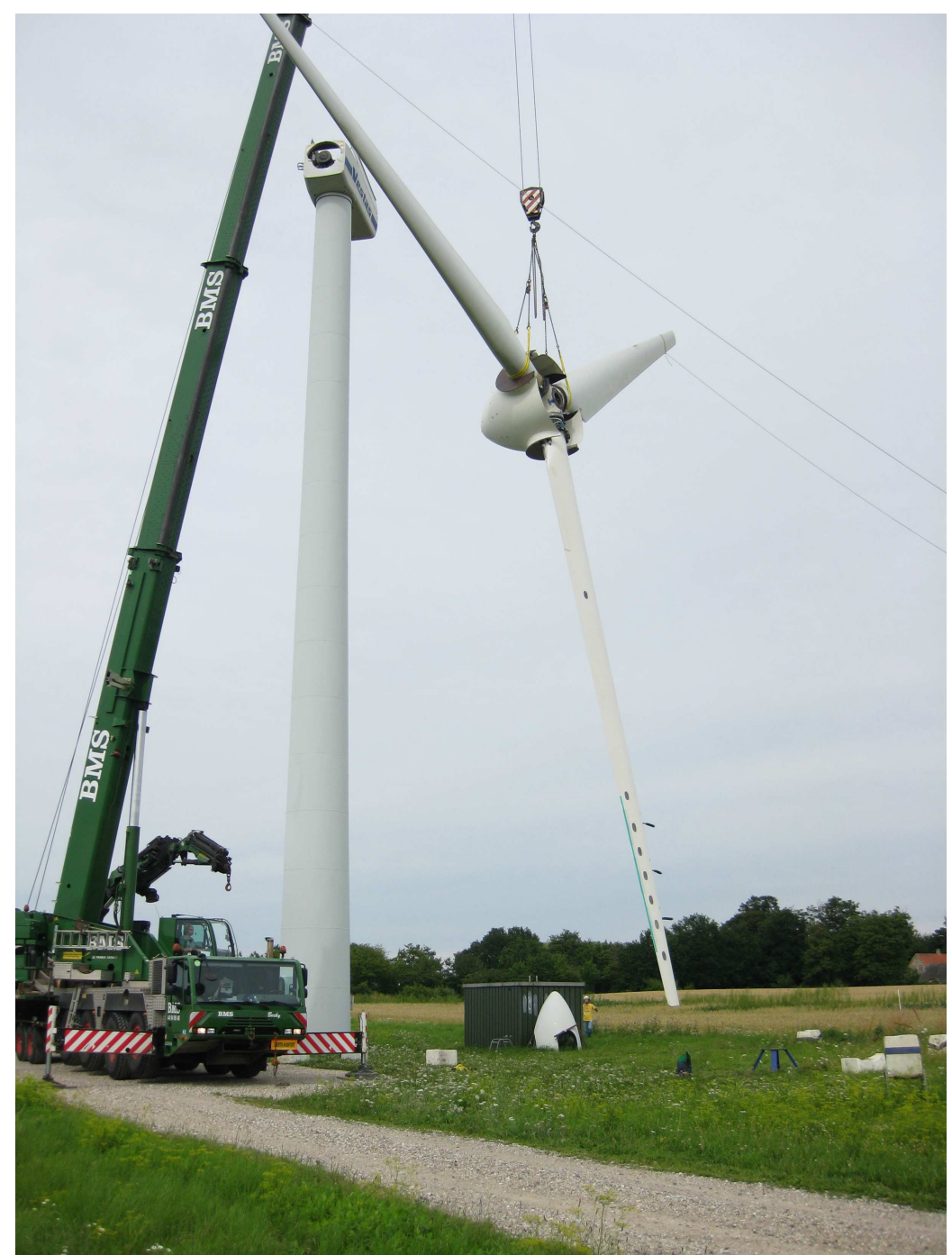

Figure 4.1: Installation of the rotor with the trailing edge flaps on the V27 wind turbine. 


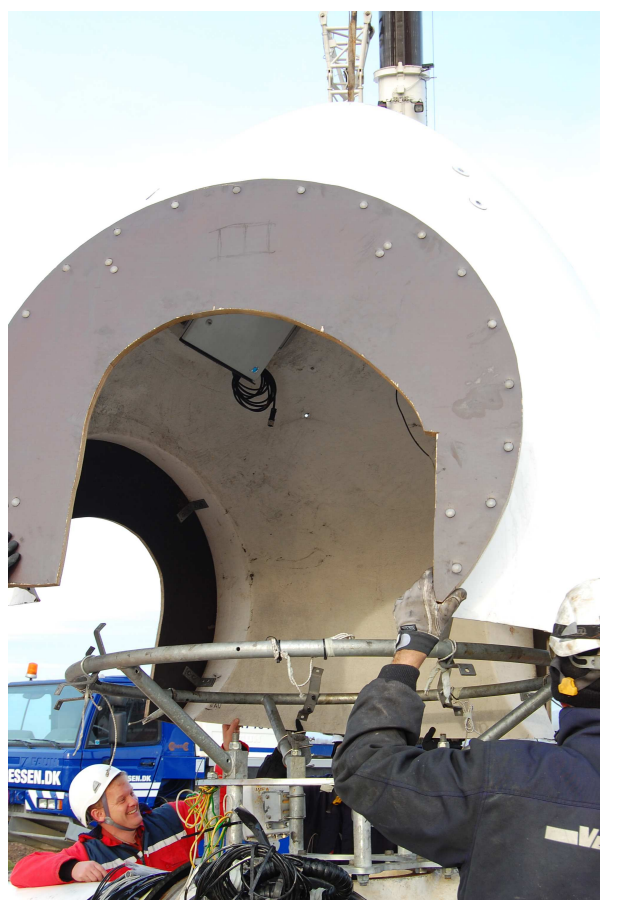

Figure 4.2: Photo of the turbine spinner. The cabinet box is fixed on the inner part of the spinner.

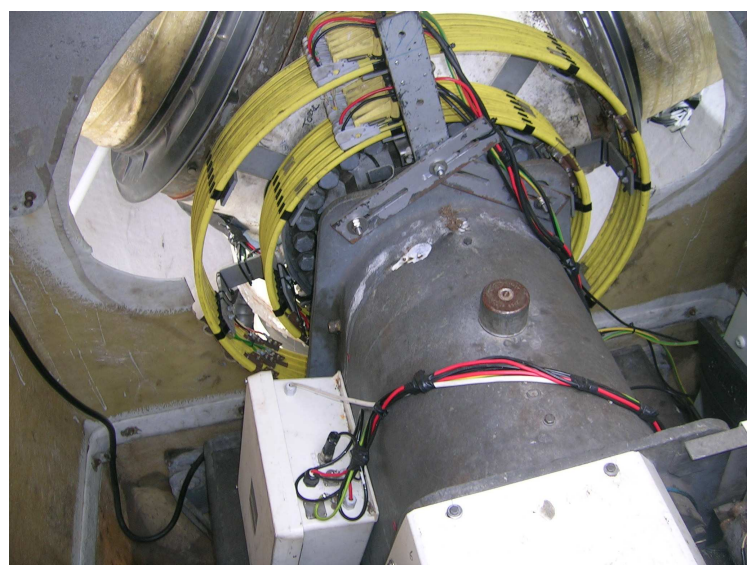

Figure 4.3: Photo of the slip ring which conducts the electricity from the power supplies in the nacelle to the cabinet box in the spinner and the actuators in the blades. 


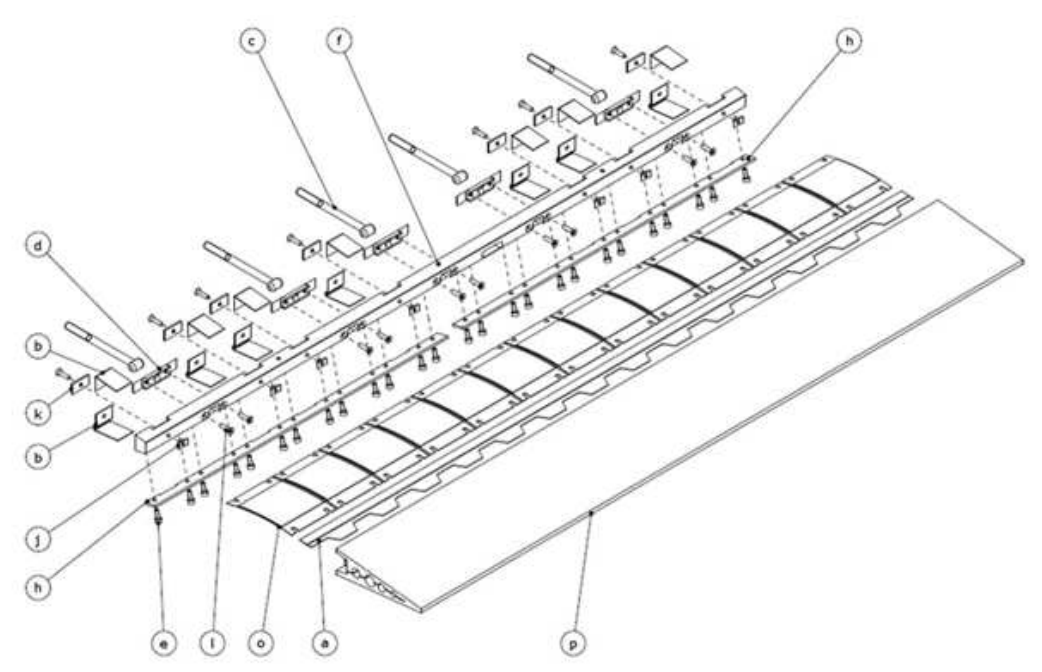

Figure 4.4: Design of the pizeo electric flap actuator.

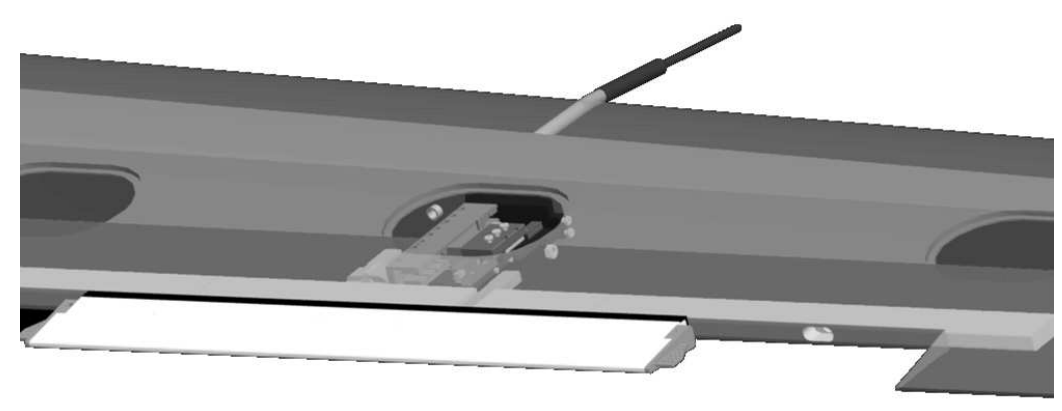

Figure 4.5: Design of the linear motor flap actuator.

point at frequencies up to the $2 \mathrm{P}$ frequency. Only results from the second set of actuators are presented in this section.

The trailing edge flaps ability to alter the flapwise blade root bending moment is not large enough to fully alleviate the flapwise blade root fatigue loads under the wind conditions of this specific site. Longer or wider trailing edge flaps, with a larger deflection would have been necessary. Both actuators were designed so that the blade with its trailing edge flaps fixed in their neutral position has the same aerodynamic properties as the two other blades. However, the implementation of the trailing edge flaps in the blade implied structural modification of the blade, especially concerning the mass distribution and the edgewise stiffness. As a consequence, the eigenfrequencies of the blade with trailing edge flaps differed slightly from the two other blades.

The demonstrator turbine was also fully instrumented for the purpose of the test (Figure 4.6). Sensors in the blade included three Pitot tubes and three sets of pressure holes at the leading edge of the blade, in front of each trailing edge flap, strain gages at the blade root, to measure flapwise and edgewise bending moments, strain gages on the actuators to measure the flap hinge 


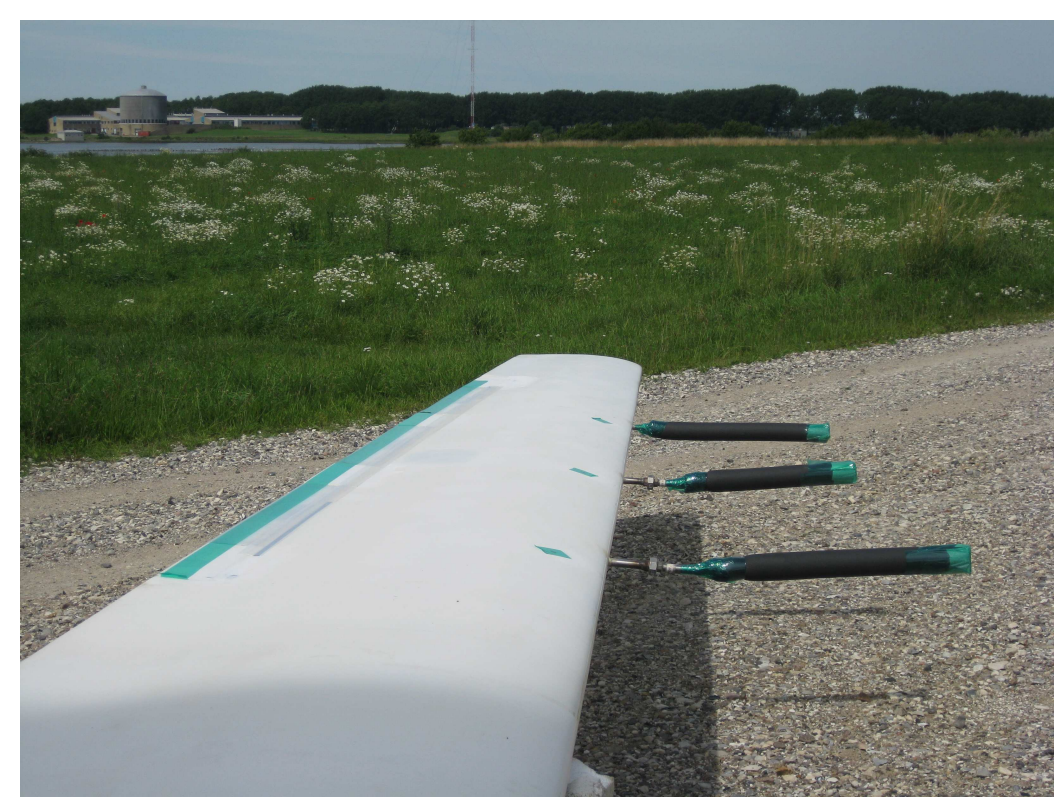

Figure 4.6: Photo of the V27 blade before being mounted on the turbine. The trailing edge flaps, the Pitot tubes and the pressure tabs are protected to avoid any damage during the installation and intrusion of water in the Pitot tubes and the pressure tabs.

moment, and some accelerometers. A pitch position sensor, strain gages on the main shaft, an accelerometer in the nacelle and strain gages at the tower top and tower bottom were also installed. A meteorological mast measuring wind speeds and wind directions at the hub height, as well as at the rotor top and bottom height is located around 70 meters west of the turbine.

Several actuators and sensors failed before running the tests with the second set of actuators. In spite of several hours spent fixing them in the nacelle, the spinner or the blade (Figure 4.7), some of them could not be repaired before running the tests. Problems were encountered with the two inner actuators, so the tests were run with the outer actuator only. The pitch position sensor failed as well, making online gain scheduling of the trailing edge flaps controller impossible. Problems were also encountered with some of the pressure transducers. At last, the meteorological conditions did not allow an accurate calibration of the blade root strain gages before the tests; approximate values were used when running the model predictive control.

\subsection{Implementation}

The controller hardware (Figure 4.8) consists in two real-time controllers (cRio 9024) and a data acquisition system (cDAQ). The high-level controller, which runs on one of the cRio 9024, includes the filtering, if necessary, of the measurement data and the open-loop and close-loop controller algorithm. The low-level controller, which runs on the second cRio 9024, is the local PID controller of the actuators. The mini PC sitting in the spinner is accessed remotely from the computer in the control room. Tests of the open-loop and closed-loop controllers is made throught a Labview graphical interface running on the mini PC.

The C++ code of the trailing edge flap controller used to compile the DLL for simulations is wrapped with two inputs and outputs functions, which make the link between the trailing edge flap controller 


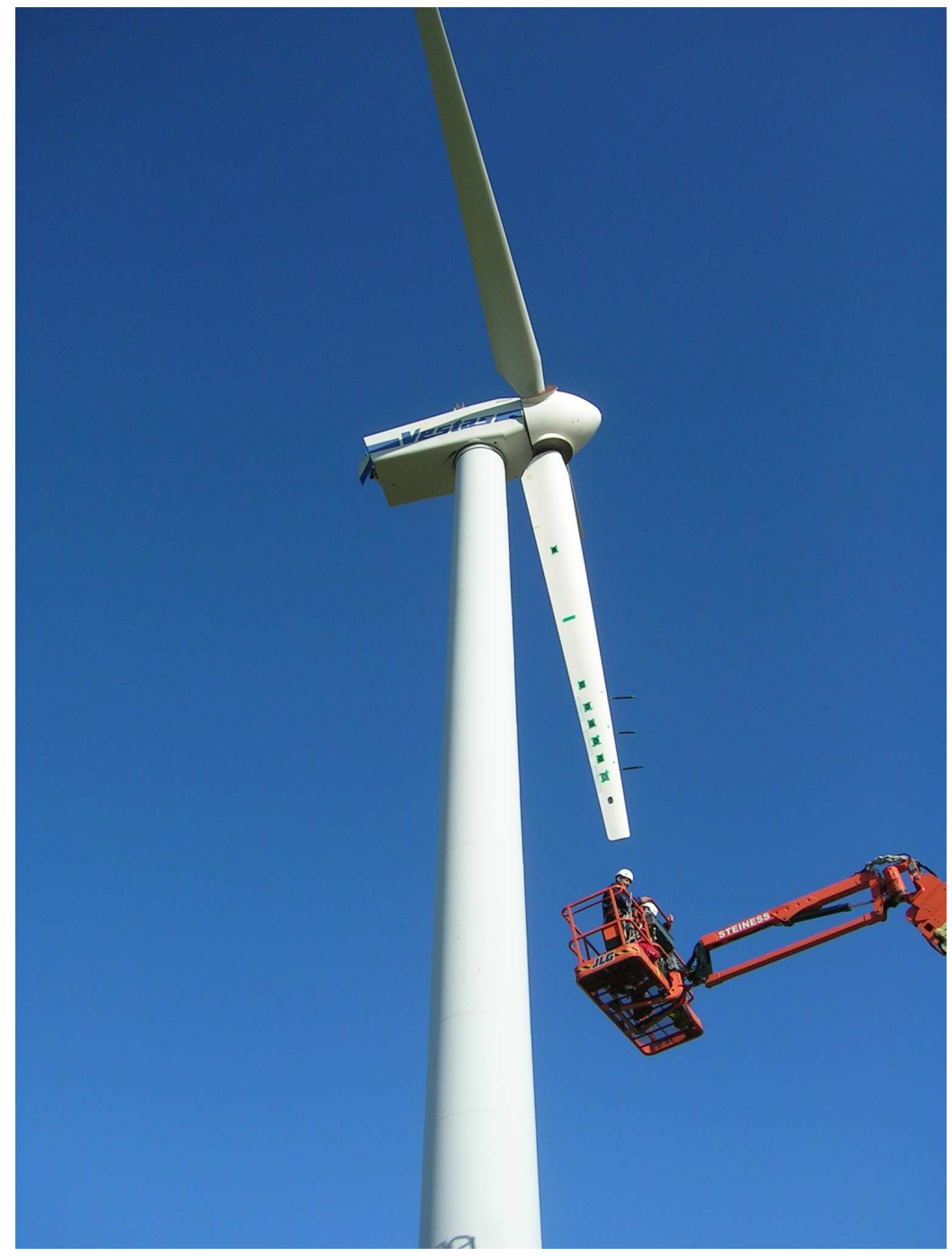

Figure 4.7: Photo of the V27 demonstrator turbine. Before running the tests, the rotor is locked with the blade with trailing edge flaps pointed downwards in order to allow access to the blade for checking the actuators and sensors, and remove the protections. 


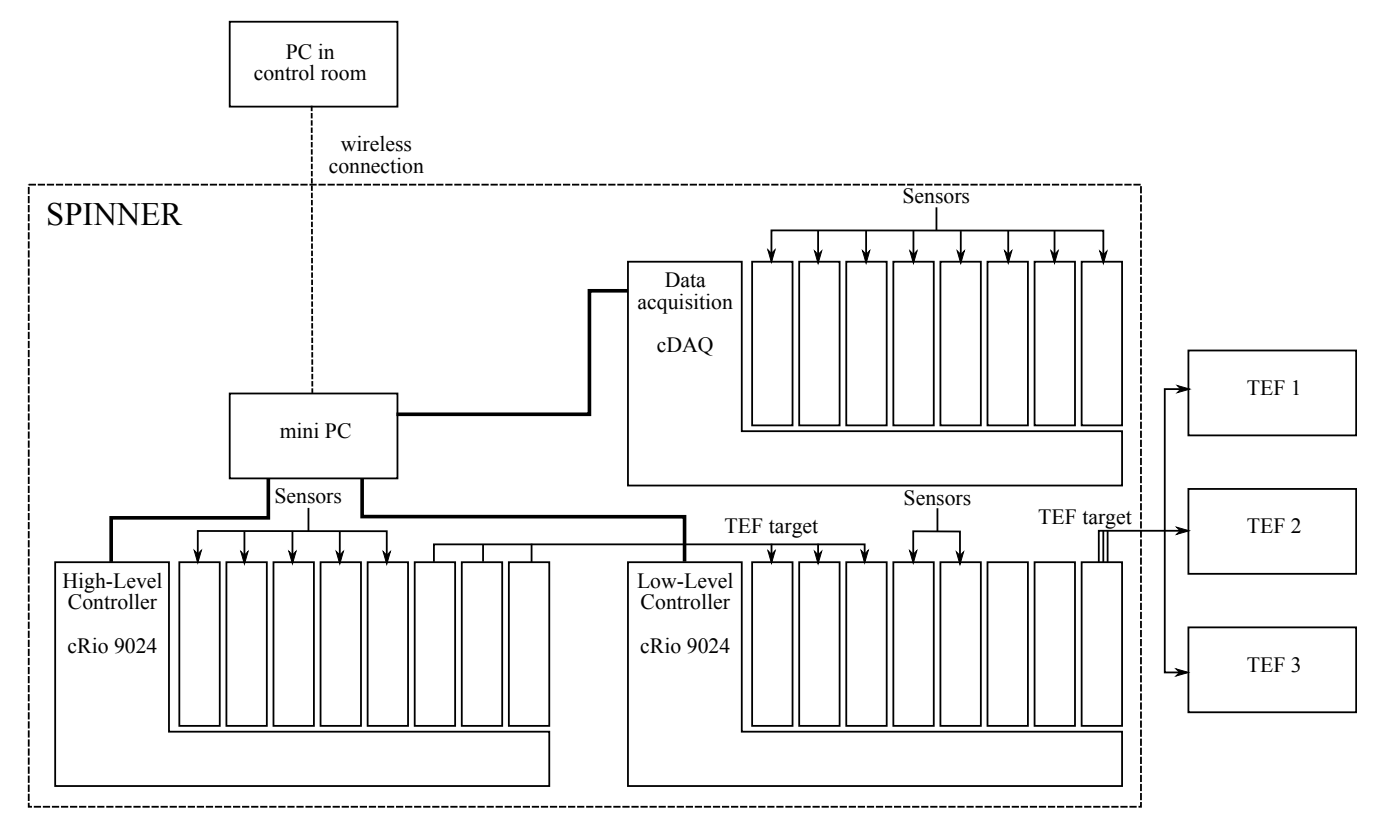

Figure 4.8: Diagram of the controller hardware configuration.

and the Labview code. Because the operating system running on the cRio is VxWorks, and not Windows, DLLs do not run on the cRio. The C++ code is instead compiled as a .out library using a C++ compiler from WindRiver [6]. The . out library is linked to the Labview code with the Call Library Function Node (Figure 4.9).

\subsection{Results}

Only results from the second actuators tests are presented in this section. Results from the first set of actuators can be found in Paper $\mathrm{E}$

\subsubsection{Measurement methodology}

Proving load reduction with mesurements is not as trivial as in simulations because of the stochastic nature of the wind.

Comparing the loads on the blade with trailing edge flaps with the load on the two other blades is not necessarily conclusive because the loads on the two blades without trailing edge flaps depend on the loads on the blade with trailing edge flaps.

It was then decided to run the tests by alternating 2-minute periods with active flaps with 2minute periods with the flaps fixed in their neutral position. Two minutes is estimated to be long enough to see the effects of the trailing edge flaps on the loads, and short enough to have consecutive time series with wind conditions similar enough to able comparison of the time series. The spectral densities of the two-minute time series with the same wind conditions and trailing edge flap controller status are then averaged together (figure 4.10). Time series during which either the data acquisition or the actuator failed are removed before postprocessing. 


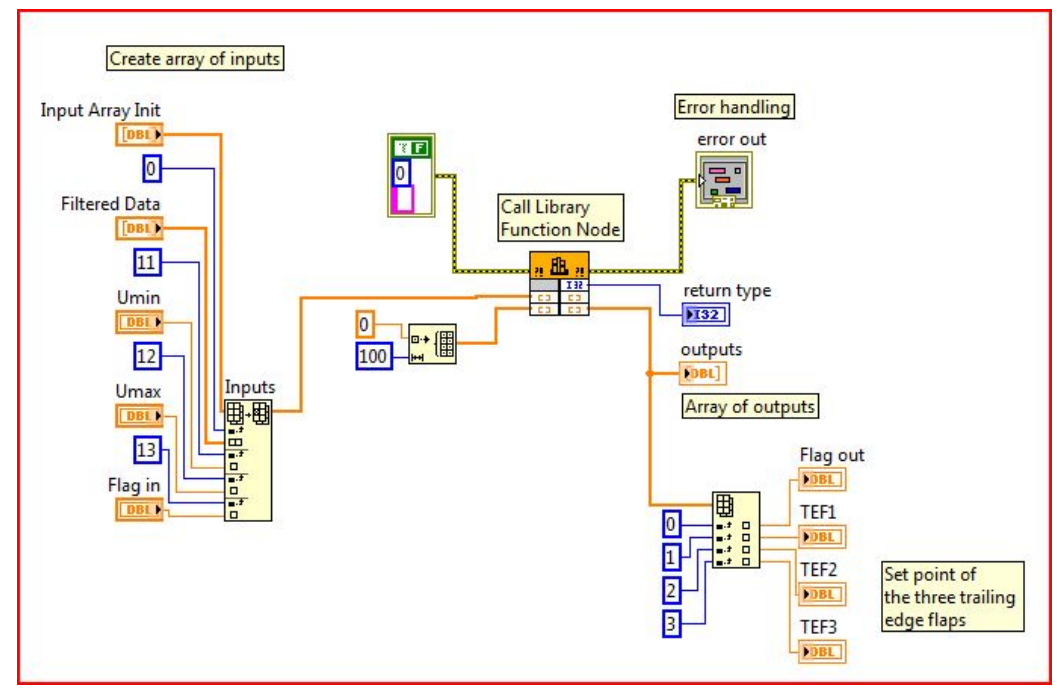

Figure 4.9: Print screen of the part of the Labview code which links the shared library where the trailing edge flaps controller is run with the Labview code.

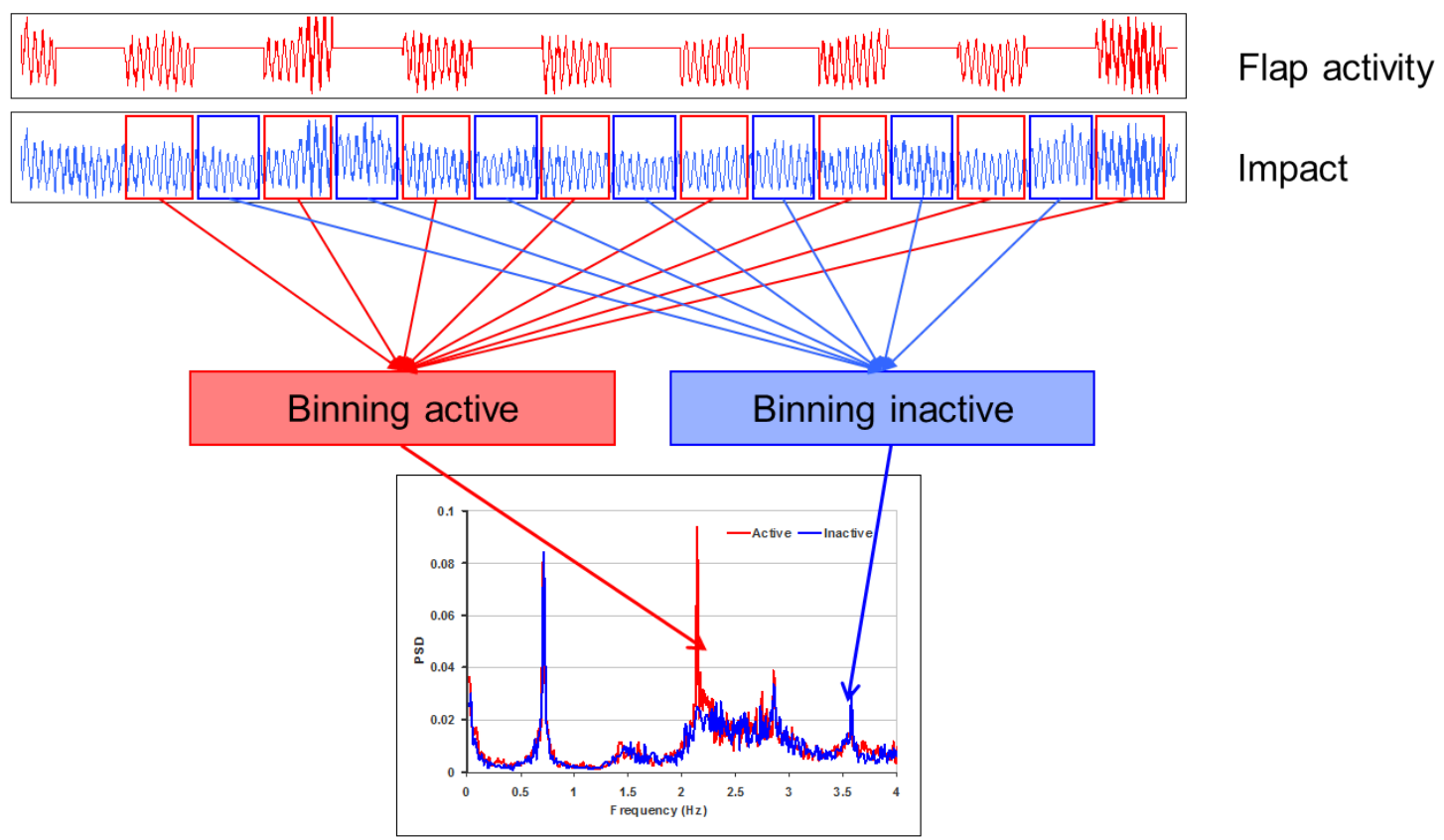

Figure 4.10: Illustration of measurement methodology used to compare spectral densities and equivalent fatigue damage when trailing edge flaps are active or fixed in their neutral position [1]. 


\subsubsection{Open loop controls}

The trailing edge flap is first actuated at fixed frequencies, between the $1 \mathrm{P}$ frequency and the first blade eigenfrequency, in order to test the system, get a better idea of the load alleviation ability of the trailing edge flap, and compare the measurements with the simulations. A clear flapwise blade root response is observed, at each frequency (Figure 4.11). Note that the test with a trailing edge flap frequency of $1.8 \mathrm{~Hz}$, close to the first blade eigenfrequency, was run at a lower trailing edge flap amplitude to avoid a too high excitation of the blade.

Comparison of the measurements with the simulations show that the trailing edge flap efficiency in the simulation model should be reduced by $20 \%$ in order to match better the measurements.

\subsubsection{Model predictive control}

The frequency-weighted model predictive control with costs on the bandpass zero-phase filtered flapwise blade root moment and on the bandstop zero-phase filtered trailing edge flap angle was tested succesully on the V27 demonstrator turbine. The controller was tuned to alleviate the $1 \mathrm{P}$ and $2 \mathrm{P}$ loads only. The controller had to be adapted in order to take into account the failed sensors: gain scheduling was no longer possible. The estimated mean free wind speed was then updated by hand, based on the measurements of the meteorological mast. The controller run at $50 \mathrm{~Hz}$.

Figures 4.12 and 4.13 show the results of a 38 minute test, consisting in 102 -minute time series with active flaps alternating with 9 2-minute time series with flaps fixed in their neutral position. The plot of the spectral density of the flapwise blade root moment with active and fixed flaps shows a clear reduction of the amplitude of the $1 \mathrm{P}$ and $2 \mathrm{P}$ loads. This reduction translates into a consistent fatigue load reduction, where the fatigue damage when flaps are active are usually between 5 and $20 \%$ lower than the previous and next time series, with fixed flaps. An average flapwise blade root fatigue load reduction of $14 \%$ is achieved during this 38 minute test.

Figure 4.14 shows results from another 64-minute test. The wind conditions during this test were different from the previous test: the wind speed was higher, close to rated power, and the wind was coming from onshore, resulting in higher wind turbulence. The meteorological mast was in the wake of the wind turbine. The study of the flapwise blade root equivalent fatigue load of those 32 2-minute time series shows that the trailing edge flap controller reduced the loads for the first 13 time series, but then increase them consistently by 5 to $15 \%$. The controller seems to work well again for the last three time series. These results clearly show a lack of robustness or adaptability of the trailing edge flap controller. Three possible reasons for explaining that the controller did not work correctly during this test were identified:

- The mean free wind speed was poorly estimated. The mean free wind speed, used for gain scheduling, was estimated from wind speed measurements from the meterological mast, which was in the wind turbine wake. The mean free wind speed was maybe underestimated by a few m.s ${ }^{-1}$.

- The pitch controller and the trailing edge flaps controller may have worked against each other. Unfortunately, the pitch position was not logged and it is thus not possible to check whether the pitch controller was influenced by the traiing edge flap controller. The study of the spectral density of the flapwise blade root moment does not show suspicious loads at frequencies others than the $1 \mathrm{P}, 2 \mathrm{P}$ and $3 \mathrm{P}$ frequencies and first flapwise eigenfrequency. 

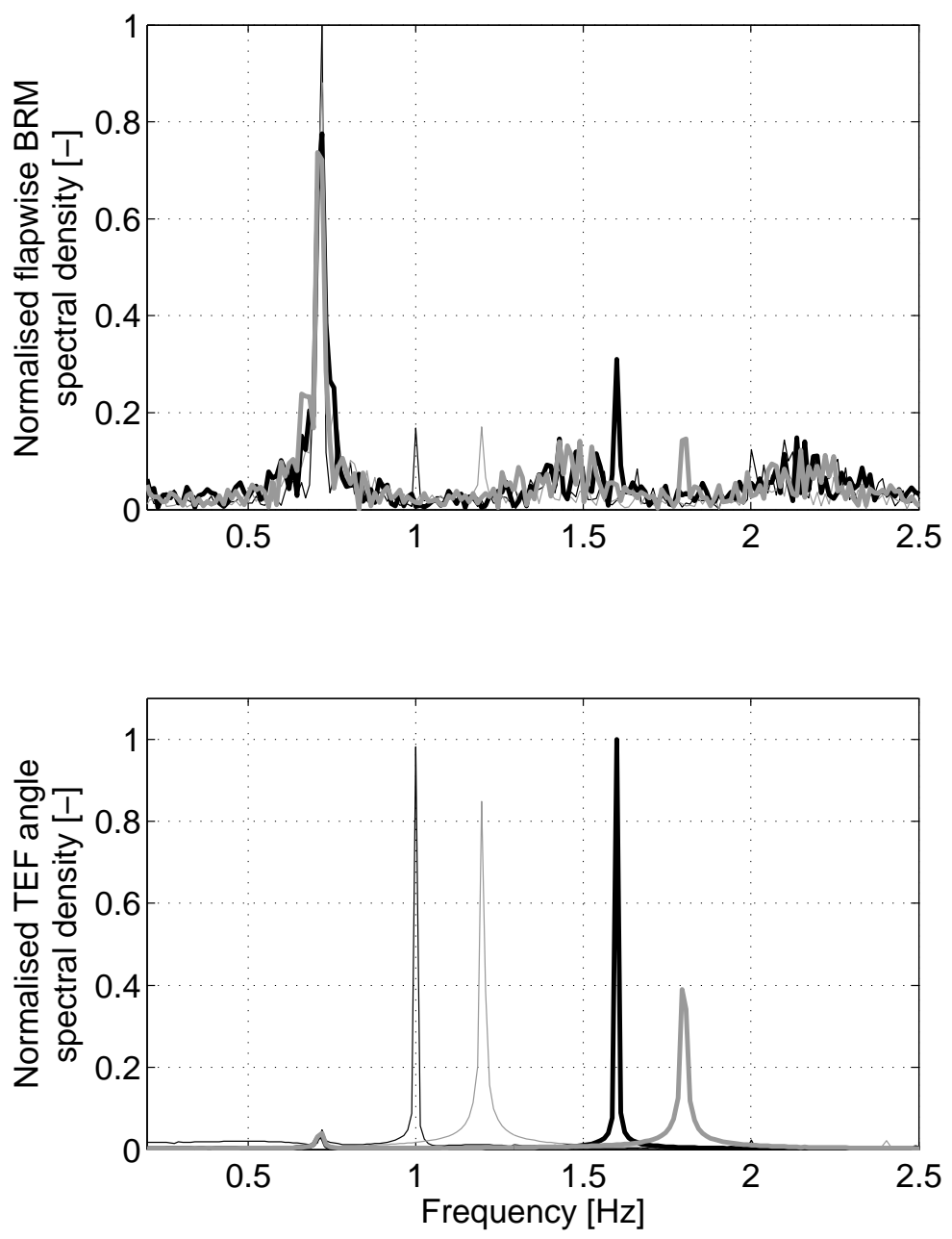

Figure 4.11: Measurements, sine actuation. Flapwise blade root moment (top) and trailing edge flap angle (bottom) spectral densities when the trailing edge flap is actuated as a sine function at frequencies of $1 \mathrm{~Hz}$ (thin black line), $1.2 \mathrm{~Hz}$ (thin grey line), $1.6 \mathrm{~Hz}$ (thick black line) and $1.8 \mathrm{~Hz}$ (thick grey line). 

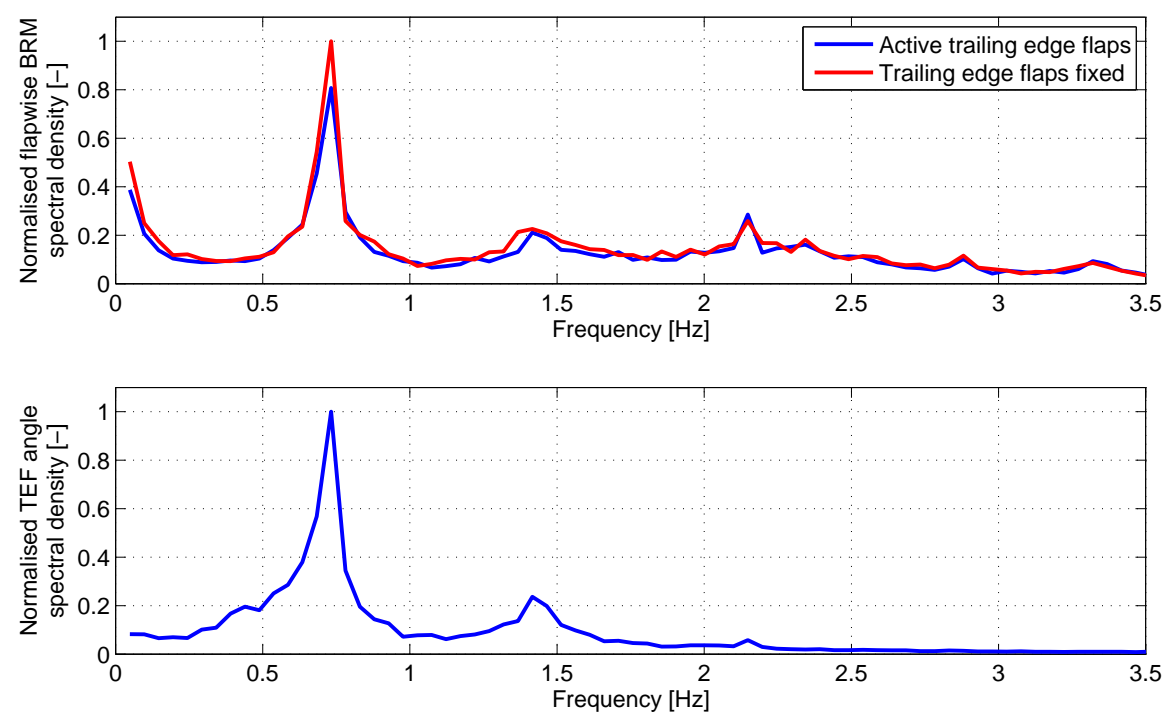

Figure 4.12: Flapwise blade root moment (top) and trailing edge flap angle (bottom) spectral densities when the trailing edge flaps are fixed in their neutral position (red) and when they are controlled with a model predictive control (blue).

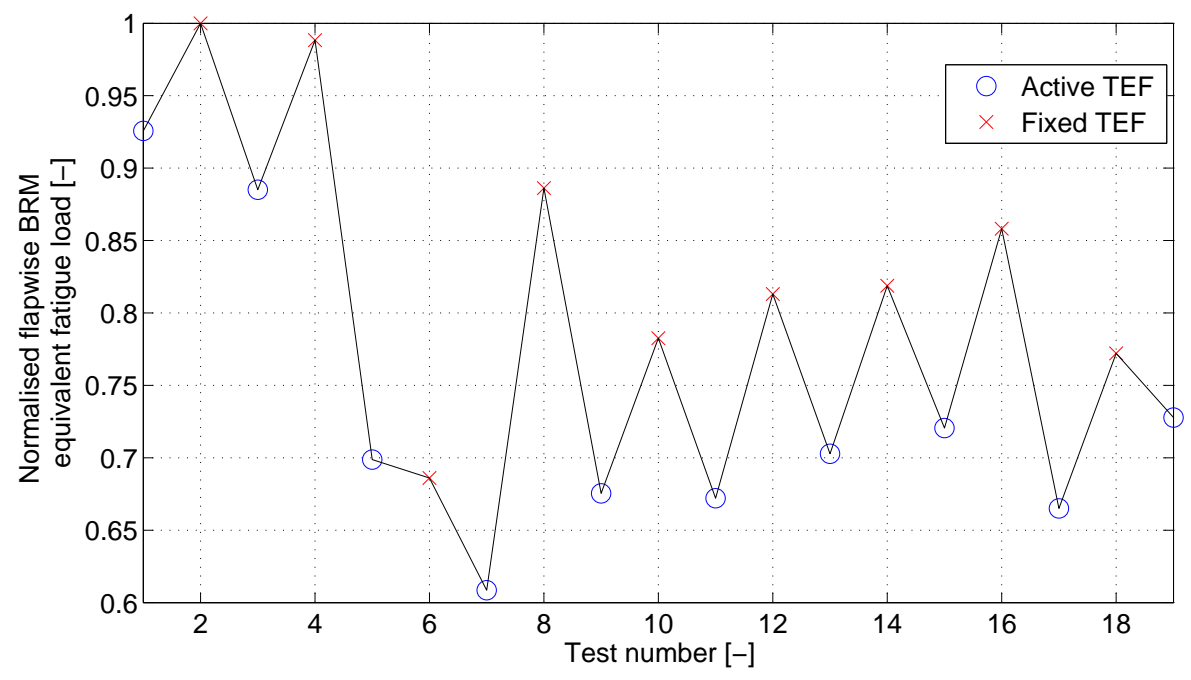

Figure 4.13: Normalised flapwise blade root equivalent fatigue load for the 19 consecutive 2-minute tests with successful load reduction. Tests with fixed trailing edge flaps are marked with red crosses, tests with active trailing edge flap are marked with blue circles. 


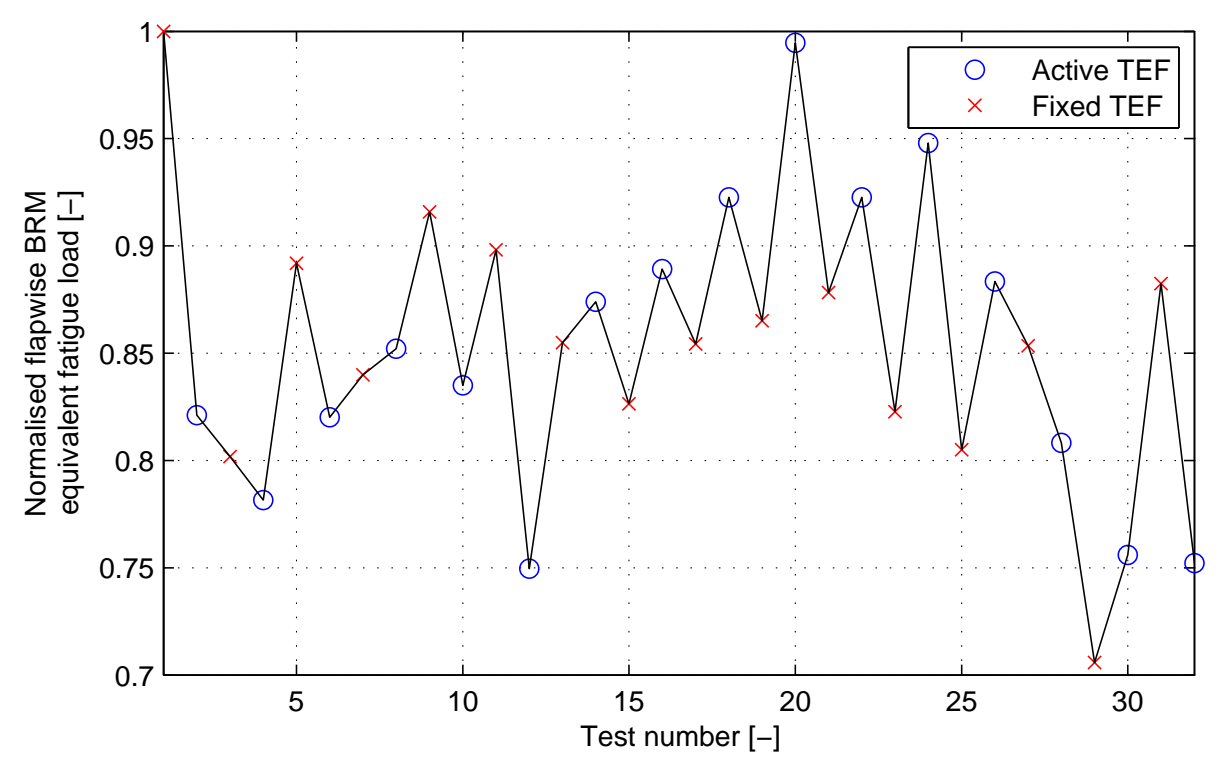

Figure 4.14: Normalised flapwise blade root equivalent fatigue load for the 32 consecutive 2-minute tests when load reduction is not always achieved. Tests with fixed trailing edge flaps are marked with red crosses, tests with active trailing edge flap are marked with blue circles.

- Most probably, the tuning of the trailing edge flap controller, and especially the Kalman filter, was not good for those specific wind conditions. It is not possible to compare directly the wind conditions when the trailing edge flap controller successed or failed reducing the flapwise blade root fatigue loads because the meteorological mast was in the wake of the wind turbine when it failed decreasing the loads. However, the spectral density of the flapwise blade root moment when the trailing edge flaps are fixed show that the amplitude of the $2 \mathrm{P}$ and $3 \mathrm{P}$ loads were significantly higher during the tests where the trailing edge flap controller failed. The standard deviation of the flapwise blade root moment was $17 \%$ higher when the controller failed than when it succeded in decreasing the loads. This indicates that the wind turbulence was significantly higher when the trailing edge flap controller failed. This test reinforces the idea that the disturbance model and the estimator should be improved. 


\section{CHAPTER 5}

\section{Conclusions and future development}

The work presented in this thesis includes the design of a trailing edge flaps controller based on frequency-weighted model predictive control, and its test both in simulations and on a full-scale experiment.

A simplified analytical linear model of a rotating blade with trailing edge flaps, which consists in only two states, is used to design the trailing edge flaps controller. I can be adapted whether Pitot tubes are available on the blade or not. This simplified linear model, from trailing edge flap angle or pitch angle to flapwise blade root bending moment, matches well the Flex5 non-linear model.

Three different approaches of frequency-weighted model predictive control are described. The two first methods keep costs of the model predictive control in the time domain: the cost function is a quadratic cost on respectively the filtered and the zero-phase filtered inputs and outputs. The inputs and outputs filters are then designed in order to increase the amplitude of the inputs and outputs frequencies which have to be targeted by the trailing edge flaps. Using zero-phase filters (forward-backward filters) instead of conventional filters improves significantly the performance of the controller, especially when high order filters with high phase lags are used. In the third method, the costs are added on the amplitude of the Fourier transform coefficients of the inputs and the outputs. The advantage of this method is to be very flexible in its way to add costs at the different frequencies. However, this method requires longer horizons, and is not suited for applications with targeted frequencies significantly smaller than the controller sampling frequency.

The use of the frequency-weighted model predictive control with costs on the zero-phase filtered predicted inputs and ouputs proved to be successful both in simulations and experiments. The controller was fast enough to be run at $50 \mathrm{~Hz}$ on a real-time cRio 9024. Introducing high-order zero-phase filters in the cost function helped the controller to target the $1 \mathrm{P}$ and $2 \mathrm{P}$ loads: the absence og high frequency content in the trailing edge flap actuation helped reducing the wear and tear of the actuators, during the full-scale test. At last, the model predictive control handled well the tight constraints on the trailing edge flap deflection.

The full-scale experiment on a Vestas V27 wind turbine equipped with three trailing edge flaps on one blade was a success. In spite of failed sensors and actuators, a consistent flapwise blade 
root fatigue load reduction was achieved. An average of $14 \%$ load reduction was reached on a 38 minute test.

The experiment, in spite of its success, also highlighted several weaknesses of the trailing edge flaps controller presented in this thesis. The controller's lack of adaptability lead to performance which were very dependent on the wind conditions, and the trailing edge flaps controller was also increasing the flapwise blade root fatigue loads during given wind conditions. Improvements should be made especially on the disturbance model and the estimator in order to make them adaptable to the local wind conditions. Reliability of the trailing edge flaps and the sensors was also an issue during the test: they are subject to harsh conditions like vibrations, water, debris in the blade, corrosion etc.

A future development of this trailing edge flap controller would be to combine it with the pitch controller and to target at the power production and at several of the wind turbine loads. Modelbased controls, and model predictive control as an example, would be very good candidates for these Multiple Inputs Multiple Outputs systems. Another research area concerns extreme loads: trailing edge flaps should be used in combination with the pitch actuators to tackle the turbine extreme loads. 


\section{Bibliography}

[1] Adaptive Trailing Edge Flap (ATEF), consolidation report.

[2] CBLAS homepage. http://www.netlib.org/blas/. accessed 05/12/2011.

[3] CVXGEN homepage. http://cvxgen.com/. accessed 16/01/2012.

[4] IEC 61400-1 Ed3: Wind turbines - part 1: Design requirements. ISBN: 978-2-88912-201-1.

[5] qpOASES homepage. http://www.kuleuven.be/optec/software/qpOASES. accessed $26 / 10 / 2011$.

[6] Wind River homepage. http://www.windriver.com/. accessed 10/01/2012.

[7] P. B. Andersen, C. Bak, M. Gaunaa, and T. Buhl. Wind tunnel test of a closed loop controller for an airfoil with trailing edge flaps. In TORQUE 2010: The Science of Making Torque from Wind, Crete, Greece, June 2010.

[8] Peter Bjørn Andersen, Mac Gaunaa, Christian Bak, and Morten Hartvig Hansen. A dynamic stall model for airfoils with deformable trailing edges. Wind Energy, 12(8):734-751, 2009.

[9] Peter Bjørn Andersen, Lars Henriksen, Mac Gaunaa, Christian Bak, and Thomas Buhl. Deformable trailing edge flaps for modern megawatt wind turbine controllers using strain gauge sensors. Wind Energy, 13(2-3):193-206, 2010.

[10] T. D. Ashwill, G. Kanaby, K. Jackson, and M. Zutech. Development of the swept twist adaptive rotor (star) blade. In Proceedings of the 48th AIAA Aerospace Sciences Meeting Including the New Horizons Forum and Aerospace Exposition, Orlando, Florida, 2010.

[11] Christian Bak, Mac Gaunaa, Peter B. Andersen, Thomas Buhl, Per Hansen, and Kasper Clemmensen. Wind tunnel test on airfoil Ris $\varnothing-\mathrm{B} 1-18$ with an active trailing edge flap. Wind Energy, 13(2-3):207-219, 2010.

[12] TK Barlas and GAM van Kuik. Review of state of the art in smart rotor control research for wind turbines. Progress in Aerospace Sciences, 46(1):1-27, 2010.

[13] S. Basualdo. Load alleviation on wind turbine blades using variable airfoil geometry. Wind Engineering, 29(2):169-182, Mar. 2005.

[14] T. Behrens and W. Jun Zhu. Feasibility of aerodynamic flap hinge moment measurements as input for load alleviation control. In EWEA 2011, Brussels, Belgium, 2011. 
[15] D. E. Berg, D. G. Wilson, B. R. Resor, M. F. Barone, and J. C. Berg. Active aerodynamic blade load control impacts on utility-scale wind turbines. In AWEA Windpower 2009, Chicago, IL, 2009.

[16] L. Bergami. Adaptive trailing edge flaps for active load reduction. Delft, Netherlands, October 2011. 7th PhD Seminar on Wind Energy in Europe, Delft University of Technology.

[17] L. Bergami and M. Gaunaa. ATEFlap aerodynamic model, a dynamic stall model including the effects of trailing edge flap deflection. Technical Report Ris $\varnothing-\mathrm{R}-1792(\mathrm{EN})$, Ris $\varnothing$ National Laboratory. Technical University of Denmark, Roskilde, Denmark, November 2011.

[18] E. A. Bossanyi. Individual blade pitch control for load reduction. Wind Energy, 6(2):119-128, 2003.

[19] E. A. Bossanyi. Further load reductions with individual pitch control. Wind Energy, 8(4):481$485,2005$.

[20] T. Buhl, M. Gaunaa, and C. Bak. Potential load reduction using airfoils with variable trailing edge geometry. Journal of Solar Energy Engineering, 127(4):503-516, November 2005.

[21] T. Burton, D. Sharpe, N. Jenkins, and E. A. Bossanyi. Wind Energy handbook. Wiley, 2008.

[22] M. Capellaro and M. Kühn. Boundaries of bend twist coupling. In TORQUE 2010: The Science of Making Torque from Wind, 2010.

[23] D. Castaignet. Analytical linear model of a blade with trailing edge flaps (for design of modelbased controls). Technical report, Vestas Wind Systems A/S, 2012. Confidential. DMS: 0027-3096.

[24] D. Castaignet. Frequency-weighted model predictive control. Technical report, Vestas Wind Systems A/S, 2012. Confidential. DMS: 0027-3068.

[25] M. Evans, M. Cannon, and B. Kouvaritakis. Linear stochastic mpc under finitely supported multiplicative uncertainty. In Wind Turbine Control Symposium. Aalborg University, Nov. 2011.

[26] H. J. Ferreau, H. G. Bock, and M. Diehl. An online active set strategy to overcome the limitations of explicit mpc. International Journal of Robust and Nonlinear Control, 18(8):816$830,2008$.

[27] M. Gaunaa. Unsteady two-dimensional potential-fl ow model for thin variable geometry airfoils. Wind Energy, 13:167-192, 2010.

[28] M. H. Hansen. Aeroelastic modal analysis of backward swept blades using HAWCStab2. Technical Report Ris $\varnothing-R-1769(\mathrm{EN})$, Ris $\varnothing$ DTU, National Laboratory for Sustainable Energy, February 2011.

[29] M. H. Hansen, M. Gaunaa, and H. A. Madsen. A Beddoes-Leishman type dynamic stall model in state-space and indicial formulations. Technical Report R-1354(EN), Risø National Laboratory, Roskilde (DK), 2004.

[30] M. H. Hansen, A. Hansen, T. J. Larsen, S. Øye, P. Sørensen, and P. Fuglsang. Control design for a pitch-regulated, variable speed wind turbine. Technical Report Ris $\varnothing-\mathrm{R}-1500(\mathrm{EN})$, Ris $\varnothing$ National Laboratory, January 2005.

[31] M. H. Hansen and I. B. Sønderby. Open- and closed-loop aero-servo-elastic analysis with hawcstab2. In M. H. Hansen, editor, Ris $\emptyset-R-1796(E N)$. Presentations from the Aeroelastic Workshop - latest results from AeroOpt, Oct. 2011. 
[32] M. O. L. Hansen. Aerodynamics of Wind Turbines. Earthscan, 2008. ISBN: 978-1-84407-438-9.

[33] L. C. Henriksen, M. H. Hansen, and N. K. Poulsen. Relinearized model predictive control of a floating wind turbine. Wind Energy. [submitted].

[34] L. C. Henriksen, M. H. Hansen, and N. K. Poulsen. Wind turbine control with constraint handling. Wind Energy. [submitted].

[35] L. C. Henriksen, N. K. Poulsen, and M. H. Hansen. Nonlinear model predictive control of a simplified wind turbine. In proceedings of the 18th IFAC World Congress, Milano, Italy, Aug. 2011.

[36] I. Houtzager, J.-W. van Wingerden, and M. Verhaegen. Predictor-based subspace identification toolbox version 0.4, 2010. http://www.dcsc.tudelft.nl/ datadriven/pbsid/.

[37] J. Jonkman, S. Butterfield, W. Musial, and G. Scott. Definition of a 5-mw reference wind turbine for offshore system development. Technical Report NREL/TP-500-38060, National Renewable Energy Laboratory (NREL), Feb. 2009.

[38] Matthew A. Lackner and Gijs van Kuik. A comparison of smart rotor control approaches using trailing edge flaps and individual pitch control. Wind Energy, 13(2-3):117-134, 2010.

[39] Torben Juul Larsen. How 2 HAWC2 the user's manual. Technical Report R-1597(EN), RisøNational Laboratory. Technical University of Denmark, 2009.

[40] Torben Juul Larsen, Helge A. Madsen, and Kenneth Thomsen. Active load reduction using individual pitch, based on local blade flow measurements. Wind Energy, 8(1):67-80, 2005.

[41] J. G. Leishman. Unsteady lift of a flapped airfoil by indicial concepts. Journal of Aircraft, 31(2):288-297, March-April 1994.

[42] J. M. Maciejowski. Predictive Control with Constraints. Prentice-Hall, 2002.

[43] J. F. Manwell, J.G. McGowan, and A. L. Rogers. Wind energy explained. Theory, Design and Application. John Wiley \& Sons Ltd, 2008. ISBN: 13:978-0-471-49972-5.

[44] J. Mattingley and S. Boyd. Real-time convex optimization in signal processing. Signal Processing Magazine, IEEE, 27(3):50 -61, may 2010.

[45] D. Q. Mayne, J. B. Rawlings, C. V. Rao, and P. O. M. Scokaert. Constrained model predictive control: Stability and optimality. Automatica, 36:789-814, 2000.

[46] K. Z. Østergaard, P. Brath, and J. Stoustrup. Estimation of effective wind speed. Journal of Physics: Conference Series, 75(1), 2007.

[47] S. Øye. Dynamic stall simulated as time lag of separation. In EWEC 1994, Thessaloniki, Greece, October 1994.

[48] S. Øye. Flex 4 - Simulation of wind turbine dynamics. In Proceedings of the 28 ${ }^{\text {th }}$ IEA Meeting of Experts "State of the Art of Aeroelastic Codes for Wind Turbine Calculations", pages 71-76, Technical University of Denmark, Lyngby, Denmark, April 11-12 1996.

[49] J. A. Paquette and P. S. Veers. Increased rotor size through passive load control and weight reduction concepts. In EWEC 2009, Marseille, France, 2009.

[50] Justin K. Rice and Michel Verhaegen. Robust and distributed control of a smart blade. Wind Energy, 13(2-3):103-116, 2010.

[51] K. Selvam, S. Kanev, J. W. van Wingerden, T. van Engelen, and M. Verhaegen. Feedbackfeedforward individual pitch control for wind turbine load reduction. International Journal of Robust and Nonlinear Control, 19(1):72-91, 2009. 
[52] A. A. Shabana. Flexible multibody dynamics: review of past and recent developments. Multibody system dynamics, 1(2):189-222, 1997.

[53] T. Theodorsen. General theory of aerodynamical instability and the mechanism of flutter. Technical Report Report 496, NACA, 1935.

[54] Sven Creutz Thomsen, Henrik Niemann, and Niels Kjølstad Poulsen. Robust stability in constrained predictive control through the youla parameterisations. International Journal of Control, 84(4):653-664, 2011.

[55] N. Troldborg. Computational study of the risø-b1-18 airfoil with a hinged flap providing variable trailing edge geometry. Wind Engineering, 29(2):89-113, 2005.

[56] C P van Dam, R Chow, J R Zayas, and D E Berg. Computational investigations of small deploying tabs and flaps for aerodynamic load control. Journal of Physics: Conference Series, 75(1):012027, 2007.

[57] G. J. Van der Veen, J.-W. van Wingerden, and M. Verhagen. Closed-loop system identification of wind turbines in the presence of periodic effects. In $3^{\text {rd }}$ conference on the science of making torque from wind, Heraklion, Greece, 2010.

[58] J.-W. van Wingerden, A. Hulskamp, T. Barlas, I. Houtzager, H. Bersee, G. van Kuik, and M. Verhaegen. Two-degree-of-freedom active vibration control of a prototyped smart rotor. Control Systems Technology, IEEE Transactions on, 19(2):284 -296, march 2011.

[59] J. W. van Wingerden, A. W. Hulskamp, T. Barlas, B. Marrant, G. A. M. van Kuik, D.-P. Molenaar, and M. Verhaegen. On the proof of concept of a smart wind turbine rotor blade for load alleviation. Wind Energy, 11(3):265-280, 2008.

[60] D. G. Wilson, D. E. Berg, M. F. Barone, J. C. Berg, B. R. Resor, and D. W. Lobitz. Active aerodynamic blade control design for load reduction on large wind turbines. In $E W E C$ 2009, Marseille, France, March 2009. 


\section{Paper A}

Model Predictive Control of Trailing Edge Flaps on a Wind Turbine blade

Paper published in the proceedings of the American Control Conference, June 29 - July 01, 2011, San Francisco, CA, USA. 


\title{
Model Predictive Control of Trailing Edge Flaps on a Wind Turbine blade
}

\author{
Damien Castaignet, Niels K. Poulsen, Thomas Buhl and Jens Jakob Wedel-Heinen
}

\begin{abstract}
Trailing Edge Flaps on wind turbine blades have been studied in order to achieve fatigue load reduction on the turbine components. We show in this paper how Model Predictive Control can be used to do frequency weighted control of the trailing edge flaps in order to reduce fatigue damage on the blade root. The design model is based on a modal model of the blade structure and a steady state aerodynamic model of the blade airfoils. Depending on the output filter, loads within different frequency range are decreased. A fine tuning of the Kalman filter and of the cost function allows to decrease significantly the blade root loads without damaging excessively the trailing edge flap actuators.
\end{abstract}

\section{INTRODUCTION}

Wind turbines rotor size has increased significantly over the last years in order to harvest more energy and to reduce the cost of wind energy. A large part of modern wind turbines have now blades longer than $40 \mathrm{~m}$. This increase in blades size results in an increase in both fatigue and extreme loads in the main components of the turbine: blades, drive train, tower, foundations etc. Decreasing those loads is important in order to keep the cost of energy low.

Some modern Megawatt size turbines use cyclic pitch or individual pitch control in order to alleviate some of these loads [1], [2]. Larsen et al. [1] showed that blade flap fatigue loads can be reduced by $28 \%$ using individual pitch control. Since 2003, Trailing Edge Flaps (TEF) have been studied as a possible way to alleviate even more loads [3], [4]. Those actuators have the advantage of controlling the flow locally, where it has the most impact on the loads [5]. They can also be actuated at a higher frequency than the pitch system. Wind tunnel experiments on a 2D section of a blade equipped with active trailing edge flaps [6], [7] and on a scaled rotating two-bladed smart rotor [8] confirm their potential . At last, a full scale test was carried out on a Vestas V27 turbine at Risø DTU [9].

Several control strategies have been investigated. Lackner et al. designed a PID Individual Flap Control based on the Individual Pitch Control scheme, using the Coleman transformation to make the system linear time invariant [10]. Van Wingerden et al. used subspace system identification to fine tune the PD controller used in their wind tunnel test [7]. Rice et al. focused on a robust and distributed control in order

This work is part of the ATEF project, which is partially funded by Danish National Advanced Technology Foundation (Højteknologifonden)

D. Castaignet and J.J. Wedel-Heinen are with Vestas Wind Systems A/S, Global Research, Roskilde, Denmark daca@vestas. com

N. K. Poulsen is with the Technical University of Denmark, DTU Informatics, Lyngby, Denmark

T. Buhl is with Risø DTU, National Laboratory for Sustainable Energy, Roskilde, Denmark

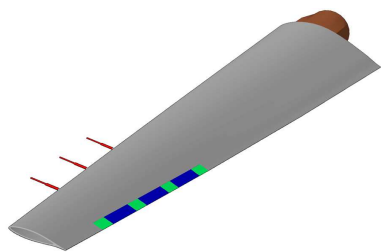

Fig. 1. Illustration of the Vestas V27 blade equipped with trailing edge flaps (blue) and Pitot tubes (red)

to ensure stability of the controller despite non linearities and model mismatch [11]. At last, Wilson et al. designed PD feedback controllers based on tip deflection or tip deflection rate, and showed a decrease in the standard deviation of the root flap bending moments [12].

This paper shows the design of a Model Predictive Control (MPC) in order to do frequency weighted control on the blade root flap bending moment variations. The system is based on the experimental V27 turbine located at Ris $\varnothing$ DTU and equipped with 3 independent trailing edge flaps [9].

\section{Model/System}

\section{A. System}

The Vestas V27 turbine is a pitch controlled horizontal axis wind turbine, with a nominal power of $225 \mathrm{~kW}$, and 13 meter long blades. The model predictive control described in this paper is meant to be tested in the future on the V27 turbine located at Risø DTU, National Laboratory for Sustainable Energy, Roskilde, Denmark. The turbine has been equipped with trailing edge flaps and extra sensors for the purpose of the ATEF project. One of the 3 blades is equipped with 3 independent trailing edge flaps (Fig. 1). Among the sensors available for this experiment are 3 Pitot tubes at the leading edge of the blade, and strain gages at the blade root [9].

The pitch control of the V27 turbine is unchanged. The controller described in this paper only actuates the trailing edge flaps. It aims at decreasing the blade root fatigue loads, while the pitch controller regulates the power production.

The configuration of actuators and sensors used in this paper matches the experimental blade available within this project.

\section{B. Non-linear model}

The design model of the model predictive control includes only the structural and the aerodynamic model of the blade. This model is based on the non-linear aero-elastic model of 
a wind turbine developed at DTU Mechanical Engineering and implemented in the aero-elastic code Flex [13].

1) Structural model: The blade structure is modeled by its mode shapes. It takes into account the blade deflection in 2 directions: flapwise and edgewise. If the $N_{g}$ first mode shapes of the blade are considered, then the flapwise and edgewise deflections of the blade section $x$ at time $t$, respectively $u_{z}(x, t)$ and $u_{y}(x, t)$, are:

$$
u_{z}(x, t):=\sum_{i=1}^{N_{g}} g_{i}(t) u_{z}^{i}(x), u_{y}(x, t):=\sum_{i=1}^{N_{g}} g_{i}(t) u_{y}^{i}(x)
$$

$u_{z}^{i}(x)$ and $u_{y}^{i}(x)$ are respectively the flapwise and edgewise deflection of the blade section $x$ under mode shape $i . g_{i}(t)$ are the generalised coordinates associated to mode shape $i$. A generalised mass $M_{g_{i}}$, stiffness $K_{g_{i}}$ and damping $C_{g_{i}}$ are associated to each of the mode shapes. The torsion mode of the blade is neglected in this model due to the high torsion stiffness of the V27 blade.

Newton's second law applies to each of the generalised modes: $M_{g_{i}} \ddot{g}_{i}+C_{g_{i}} \dot{g}_{i}+K_{g_{i}} g_{i}=F_{g_{i}} . F_{g_{i}}$, the generalised force for mode shape $i$, is the work done by the external loads (aerodynamic, centrifugal and gravity loads) on this mode shape: $F_{g_{i}}=\int p_{z}(x) u_{z}^{i}(x) d x+\int p_{y}(x) u_{y}^{i}(x) d x$, where $p_{z}(x)$ and $p_{y}(x)$ are the external loads, respectively flapwise and edgewise, acting on blade section $x$.

2) Aerodynamic model: The aerodynamic forces on an airfoil with a trailing edge flap depend on the local wind speed $\mathbf{V}_{\mathbf{r}}(x, t)$, the local angle of attack $\alpha(x, t)$ and the trailing edge flap angle $\beta(x, t)$. The relative wind speed $\mathbf{V}_{\mathbf{r}}(x, t)$ seen by the airfoil is calculated as $\mathbf{V}_{\mathbf{r}}(x, t):=$ $\mathbf{V}_{\mathbf{0}}(x, t)+\mathbf{W}(x, t)-\mathbf{V}_{\mathbf{b}}(x, t)$, where $\mathbf{V}_{\mathbf{0}}$ is the free wind speed, $\mathbf{W}$ the induced velocities and $\mathbf{V}_{\mathbf{b}}$ the blade velocity. The induced velocities depend on the thrust generated on the rotor by the aerodynamic forces. They are derived from the unsteady Blade Element Momentum [13].

At last, a dynamic stall model is used in order to derive the dynamic lift and drag dynamic coefficients, $C_{L}$ and $C_{D}$, of the airfoil with a given angle of attack $\alpha$, a trailing edge flap angle $\beta$ and a relative wind speed $\mathbf{V}_{\mathbf{r}}$. The dynamic stall model used in this paper is the one derived by Andersen and al. [14]. The flapwise and edgewise aerodynamic loads, $p_{z}^{a e r o}(x)$ and $p_{y}^{\text {aero }}(x)$, are derived from the lift and drag coefficients:

$p_{z}^{\text {aero }}(x):=\frac{1}{2} \rho\left|\mathbf{V}_{r}\right|^{2} C(x)\left(C_{L}(x) \cos (\alpha)+C_{D}(x) \sin (\alpha)\right)$,

$p_{y}^{\text {aero }}(x):=\frac{1}{2} \rho\left|\mathbf{V}_{r}\right|^{2} C(x)\left(-C_{L}(x) \sin (\alpha)+C_{D}(x) \cos (\alpha)\right)$,

where $\rho$ is the air density, and $C(x)$ the airfoil chord length.

3) Differential equations: Finally, the system of differential equations modeling the blade structure is, for each mode shape $j$ :

$$
M_{g_{j}} \ddot{g}_{j}+C_{g_{j}} \dot{g}_{j}+K_{g_{j}} g_{j}=F_{g_{j}}\left(g_{i}, \dot{g}_{i}, \omega, \varphi, \mathbf{V}_{0}, \mathbf{W}, \beta_{j}\right) .
$$

The differential equations relative to the dynamic stall model and the induced velocities can be found in [14] and [13].
4) Blade root moment: The flap blade root moment, measured by a strain gage, is $M_{z 5}:=E I \frac{\partial^{2} u_{z}(x, t)}{\partial x^{2}}$, where $E$ is the blade root's modulus of elasticity, flapwise, and $I$ its moment of inertia.

\section{Linear model}

Some simplifications are made on the non-linear model previously described before it is linearised.

- Only the first mode of the blade (dominated by flapwise deflections) is taken into account. The V27 blade is small and stiff, and simulations and measurements show that the second mode is hardly excited.

- The rotor speed is considered constant; the Vestas V27 turbine generator is fixed speed.

- Aerodynamic lags are also neglected. The Vestas V27 is a pitch controlled wind turbine. In normal production, the flow around the airfoils stays attached and aerodynamic lags are then negligible.

- Induced velocities, which have a slow dynamic, are considered constant.

- Relative wind speeds $\mathbf{V}_{r}(x, t)$ can not be measured at each point of the blade. 3 Pitot tubes along the blade measure the local wind speeds in the airfoil plan. Those measurements $\mathbf{V}_{\mathbf{P}}$ are interpolated along the blade.

The system of differential equations (1) is simplified and linearised at a given steady-state point. The upperscript 0 refers to the steady-state point values and tilded variables are the difference between a variable and its steady-state value: $\tilde{F}_{g_{i}}=F_{g_{i}}-F_{g_{i}}^{0} . \beta$ is the vector of the TEF angles. $\nabla_{x}=$ $\frac{\partial \tilde{F}_{g_{1}}}{\partial x}$ are the gradients of the generalised force for mode shape 1 . The linearised generalised force for the first mode shape is then:

$$
\tilde{F}_{g_{1}}=\nabla_{\tilde{g}_{1}} \tilde{g}_{1}+\nabla_{\tilde{g}_{1}} \dot{\tilde{g}}_{1}+\nabla_{\tilde{\varphi}} \tilde{\varphi}+\nabla_{\tilde{\beta}} \tilde{\beta}+\nabla_{\tilde{\mathbf{V}}_{\mathbf{P}}} \tilde{\mathbf{V}}_{\mathbf{P}}
$$

The differential equation (1) becomes

$$
\begin{aligned}
M_{g_{1}} \ddot{\tilde{g}}_{1}+C_{g_{1}} \dot{\tilde{g}}_{1}+K_{g_{1}} \tilde{g}_{1}= & \nabla_{g_{1}} \tilde{g}_{1}+\nabla_{\dot{\tilde{g}}_{1}} \dot{\tilde{g}}_{1} \\
& +\nabla_{\tilde{\varphi}} \tilde{\varphi}+\nabla_{\tilde{\beta}} \tilde{\beta}+\nabla_{\tilde{\mathbf{V}}_{\mathbf{P}}} \tilde{\mathbf{V}}_{\mathbf{P}},
\end{aligned}
$$

and the blade root flap moment $\tilde{M}_{f}=E I \frac{\partial^{2} u_{z}^{1}(x)}{\partial x^{2}} \tilde{g}_{1}$. Written as a state-space form, the blade model is:

$$
\dot{\mathbf{x}}=\mathbb{A} \mathbf{x}+\mathbb{B} \mathbf{u}+\mathbb{G} \mathbf{d}, \mathbf{y}=\mathbb{C} \mathbf{x}, \mathbf{z}=\mathbb{C}_{m} \mathbf{x},
$$

where the state vector $\mathbf{x}:=\left(\begin{array}{c}\tilde{g}_{1} \\ \dot{\tilde{g}}_{1}\end{array}\right)$, the input vector $\mathbf{u}:=\tilde{\beta}$, the measured disturbance vector $\mathbf{d}:=\left(\begin{array}{c}\tilde{\varphi} \\ \tilde{\mathbf{V}}_{\mathbf{P}}\end{array}\right)$, the output vector $\mathbf{y}:=\left(\tilde{M}_{f}\right)$, the measurement vector $\mathbf{z}:=\left(\tilde{M}_{f}\right)$ and the state space matrices are

$$
\begin{aligned}
& \mathbb{A}:=\left[\begin{array}{cc}
0 & 1 \\
M_{g_{1}}^{-1}\left(\nabla_{\tilde{g}_{1}}-K_{g_{1}}\right) & M_{g_{1}}^{-1}\left(\nabla_{\dot{\tilde{g}}_{1}}-C_{g_{1}}\right)
\end{array}\right], \\
& \mathbb{B}:=M_{g_{1}}^{-1} \nabla_{\tilde{\beta}} \\
& \mathbb{G}:=\left[\begin{array}{ll}
M_{g_{1}}^{-1} \nabla_{\tilde{\varphi}} & M_{g_{1}}^{-1} \nabla_{\tilde{\mathbf{V}}_{\mathbf{P}}}
\end{array}\right] \\
& \mathbb{C}:=\mathbb{C}_{m}=\left[\begin{array}{ll}
E I \frac{\partial^{2} u_{z}^{1}(x)}{\partial x^{2}} & 0
\end{array}\right] .
\end{aligned}
$$


This system is both observable and controllable.

\section{MODEL PREDICTIVE CONTROL}

\section{A. Objective}

The controller's objective is to reduce the fatigue loads at the blade root, or in other terms, to maximise the life time of the blade root. The fatigue damage of a blade is estimated by the equivalent number of cycles it has been through. The calculation of this equivalent number of cycles is based on the Rainflow counting of the loads history and the Wöhler curve of the material. This method can not be used to design the Model Predictive Control. But [15] showed the relation between the fatigue damage and the spectral properties of the loads. The controller's objective is then to reduce the amplitude of the blade root moment variations at given frequencies.

\section{B. Model}

The linearized system (2) is, for the purpose of control design, discretized at the sampling frequency $(50 \mathrm{~Hz})$. In order to handle uncertainties and disturbances, the model is augmented with noise terms. It is assumed that both the process $\left(\mathbf{w}_{k}\right)$ and the measurement noise $\left(\nu_{k}\right)$ are sequences of zero mean, Gaussian white noise: $\mathbf{w}_{k} \in \mathcal{N}\left(0, R_{1}\right)$ and $\nu_{k} \in \mathcal{N}\left(0, R_{2}\right)$.

$$
\begin{aligned}
\mathbf{x}_{k+1} & =\mathbb{A}^{d} \mathbf{x}_{k}+\mathbb{B}^{d} \mathbf{u}_{k}+\mathbb{G}^{d} \mathbf{d}_{k}+\mathbf{w}_{k}, \\
\mathbf{y}_{k} & =\mathbb{C}^{d} \mathbf{x}_{k}, \mathbf{z}_{k}=\mathbb{C}_{m}^{d} \mathbf{x}_{k}+\nu_{k}
\end{aligned}
$$

The aero-elastic code Flex uses the same modal approach as the linear model of the blade described previously. So the covariances $R_{1}$ and $R_{2}$ can be estimated by comparing the model output with simulation results with same inputs. Those covariances can then be tuned by running simulations with different values.

Basically we will control the trailing edge flaps in a LQ manner, but will emphasise some frequency regions in order to reduce the loads. For that purpose, we introduce some filters in the cost function:

$$
\begin{aligned}
& \mathbf{x}_{k+1}^{\breve{y}}=\mathbb{A}^{\breve{y}} \mathbf{x}_{k}^{\breve{y}}+\mathbb{B}^{\breve{y}} \mathbf{y}_{k}, \breve{\mathbf{y}}_{k}=\mathbb{C}^{\breve{y}} \mathbf{x}_{k}^{\breve{y}}+D^{\breve{y}} \mathbf{y}_{k}, \\
& \mathbf{x}_{k+1}^{\breve{u}}=\mathbb{A}^{\breve{u}} \mathbf{x}_{k}^{\breve{u}}+\mathbb{B}^{\breve{u}} \mathbf{u}_{k}, \breve{\mathbf{u}}_{k}=\mathbb{C}^{\breve{u}} \mathbf{x}_{k}^{\breve{u}}+\mathbb{D}^{\breve{u}} \mathbf{u}_{k} .
\end{aligned}
$$

At each time step $k$, knowing the initial conditions $\mathbf{x}_{0}=$ $\mathbf{x}_{k}, \mathbf{x}_{0}^{\breve{y}}=\mathbf{x}_{k}^{\breve{y}}$ and $\mathbf{x}_{0}^{\breve{u}}=\mathbf{x}_{k}^{\breve{u}}$, predicting the disturbances $D=\left[\begin{array}{lll}\mathbf{d}_{k}^{\prime} & \ldots & \mathbf{d}_{k+N-1}^{\prime}\end{array}\right]^{\prime}$ and the inputs $U=$ $\left[\begin{array}{lll}\mathbf{u}_{k}^{\prime} & \ldots & \mathbf{u}_{k+N-1}^{\prime}\end{array}\right]^{\prime}$ over the horizon length $N$, the outputs $Y=\left[\begin{array}{lll}\mathbf{y}_{k+1}^{\prime} & \cdots & \mathbf{y}_{k+N}^{\prime}\end{array}\right]^{\prime}$ and the filtered outputs $\breve{Y}=\left[\begin{array}{lll}\breve{\mathbf{y}}_{k+1}^{\prime} & \ldots & \breve{\mathbf{y}}_{k+N}^{\prime}\end{array}\right]^{\prime}$ can be predicted and the filtered inputs $\breve{U}=\left[\begin{array}{llll}\breve{\mathbf{u}}_{k+1}^{\prime} & \ldots & \breve{\mathbf{u}}_{k+N}^{\prime}\end{array}\right]^{\prime}$ can be calculated over the horizon length $N$ :

$$
\begin{aligned}
Y & =\Phi \mathbf{x}_{0}+\Gamma U+\Gamma_{d} D+\xi \\
\breve{Y} & =\Phi_{\breve{y}} \mathbf{x}_{0}^{\breve{y}}+\Gamma_{\breve{y}} Y+\breve{\xi} \\
\breve{U} & =\Phi_{\breve{u}} \mathbf{x}_{0}^{\breve{u}}+\Gamma_{\breve{u}} U
\end{aligned}
$$

where $\xi$ and $\breve{\xi}$ are zero mean, Gaussian white noise and

$$
\begin{gathered}
\Phi=\left[\begin{array}{c}
\mathbb{C} \mathbb{A}^{2} \\
\mathbb{C A}^{2} \\
\vdots \\
\mathbb{C} \mathbb{A}^{N}
\end{array}\right] \Phi_{\breve{y}}=\left[\begin{array}{c}
\mathbb{C}^{\breve{y}} \\
\mathbb{C}^{\breve{y}} \mathbb{A}^{\breve{y}} \\
\vdots \\
\mathbb{C}^{\breve{y}} \mathbb{A}^{\breve{y}^{N-1}}
\end{array}\right] \Phi_{\breve{u}}=\left[\begin{array}{c}
\mathbb{C}^{\breve{u}} \\
\mathbb{C}^{\breve{u}} \mathbb{A}^{\breve{u}} \\
\vdots \\
\mathbb{C} \mathbb{C}^{\breve{u}} \mathbb{A}^{\breve{u}^{N-1}}
\end{array}\right] \\
\Gamma=\left[\begin{array}{cccc}
\mathbb{C} & 0 & \cdots & 0 \\
\mathbb{C} \mathbb{B} & \mathbb{C B} & & \vdots \\
\vdots & & \ddots & \vdots \\
\mathbb{C} \mathbb{A}^{N-1} \mathbb{B} & \ldots & \cdots & \mathbb{C B}
\end{array}\right]
\end{gathered}
$$

$\Gamma_{d}$ is defined in a similar way as $\Gamma$.

$$
\Gamma_{\breve{y}}=\left[\begin{array}{cccc}
\mathbb{D}_{\breve{y}} & 0 & \ldots & 0 \\
\mathbb{C}_{\breve{y}} \mathbb{B}_{\breve{y}} & \mathbb{D}_{\breve{y}} & & \vdots \\
\vdots & & \ddots & \vdots \\
\mathbb{C}_{\breve{y}} \mathbb{A}_{\breve{y}}^{N-1} \mathbb{B}_{\breve{y}} & \ldots & \mathbb{C}_{\breve{y}} \mathbb{B}_{\breve{y}} & \mathbb{D}_{\breve{y}}
\end{array}\right]
$$

and $\Gamma_{\breve{u}}$ is defined in a similar way as $\Gamma_{\breve{y}}$.

\section{Cost function and constraints}

The purpose of the controller is to decrease the variations of the blade root flap moment with emphasise on some given frequencies. It is important to preserve the actuators as well, by avoiding unnecessary actuations.

The cost function $\phi$ consists in:

- a cost $\phi_{\breve{y}}$ on the filtered outputs in order to do frequency weighted control on the blade root flap moment

- a cost $\phi_{u}$ on the TEF angles

- a cost $\phi_{\breve{u}}$ on the filtered inputs so that actuation of the TEF at high frequencies can be avoided.

$$
\begin{aligned}
\phi & =\underbrace{\sum_{i=1}^{N}\left\|\breve{y}_{i}\right\|_{W_{\breve{y}}}^{2}}_{\phi_{\breve{y}}}+\underbrace{\sum_{i=1}^{N}\left\|u_{i}\right\|_{W_{u}}^{2}}_{\phi_{u}}+\underbrace{\sum_{i=1}^{N}\left\|\breve{u}_{i}\right\|_{W_{\breve{u}}}^{2}}_{\phi_{\breve{u}}} \\
& =\frac{1}{2}\left[\breve{Y}^{\prime} W_{\breve{Y}} \breve{Y}+U^{\prime} W_{U} U+\breve{U}^{\prime} W_{\breve{U}} \breve{U}\right]
\end{aligned}
$$

Combining (9), (10), (11) and (12) leads to $\Phi=\frac{1}{2} U^{\prime} H U+$ $b^{\prime} U+c$, where

$$
\begin{aligned}
H & =\Gamma^{\prime} \Gamma_{\breve{y}}^{\prime} W_{\breve{Y}} \Gamma_{\breve{y}} \Gamma+W_{U}+\Gamma_{\breve{u}}^{\prime} W_{\breve{U}} \Gamma_{\breve{u} y}, \\
b & =\Gamma^{\prime} \Gamma_{\breve{y}}^{\prime} W_{\breve{Y}}\left(\Phi_{\breve{y}} \mathbf{x}_{0}^{\breve{y}}+\Gamma_{\breve{y}}\left(\Phi x_{0}+\Gamma_{d} D\right)\right)+\Gamma_{\breve{u}}^{\prime} W_{\breve{U}} \Phi_{\breve{u}} \mathbf{x}_{0}^{\breve{u}},
\end{aligned}
$$

and $c$ is a term independent of $U$. Hard constraints are added on the TEF angles and angle rates.

$$
\mathcal{U}:=\left\{U \in\left[U_{\min }, U_{\max }\right], \Delta U \in\left[\Delta U_{\min }, \Delta U_{\max }\right]\right\},
$$

where $\Delta U$ is the vector of TEF angles difference $\left[u_{l+1}-\right.$ $\left.u_{l}\right]_{l \in[0 ; N-1]}$. Extreme flapwise bending moment is not an issue for this controller, so constraints on the states are not necessary.

So, the model predictive control consists in solving, at each time step, the quadratic program $\min _{U \in \mathcal{U}}\left(\frac{1}{2} U^{\prime} H U+b^{\prime} U\right)$. 

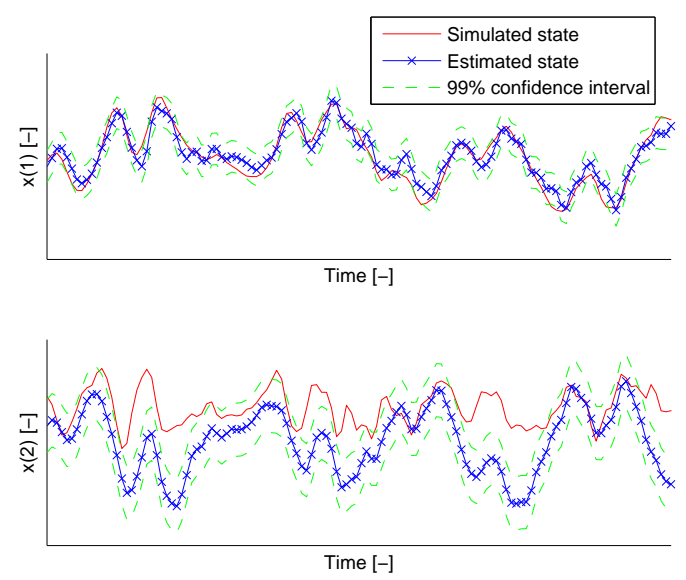

Fig. 2. Estimated (blue) and true (red) states of the system. The $99 \%$ confidence interval is indicated in dash green.

\section{Kalman filter}

Blade deflection and deflection rate are not easy to measure on a turbine. A Kalman filter is necessary to estimate the states. The predictive Kalman filter is prefered over the ordinary filter in order to ease the implementation of the controller in the real turbine.

At each time step $k, \hat{\mathbf{x}}_{k+1 \mid k}$ is the states estimates at the next time step $k+1$, based on the inputs $\mathbf{u}_{k}$, the disturbances $\mathbf{d}_{k}$ and the measurements $\mathbf{y}_{k}$ at time step $k$.

$$
\hat{\mathbf{x}}_{k+1 \mid k}=\left(\mathbb{A}^{d}-K \mathbb{C}_{m}^{d}\right) \hat{\mathbf{x}}_{k \mid k-1}+\mathbb{B}^{d} \mathbf{u}_{k}+\mathbb{G}^{d} \mathbf{d}_{k}+K \mathbf{y}_{k}
$$

where $K=\mathbb{A}^{d} P \mathbb{C}^{d^{\prime}}\left(\mathbb{C}^{d} P \mathbb{C}^{d^{\prime}}+R_{2}\right)^{-1}$ and $P$ is the solution of the discrete time algebraic Riccati equation $\mathbb{A}^{d} P \mathbb{A}^{d^{\prime}}-P-K \mathbb{C}^{d} P \mathbb{A}^{d^{\prime}}+R_{1}=0$.

Fig. 2 shows that the first state of the system, proportional to the blade deflection, is well estimated by the predictive Kalman filter and is most of the time within the $99 \%$ confidence interval. The second state, proportional to the blade deflection rate, is not predicted as well as the first state because of a plant-model mismatch. Input or output disturbance models are usually used in order to deal with this mismatch and to achieve offset-free MPC [16]. As the objective of this controller is to reduce the variance of the output, those disturbance states are not strictly needed.

\section{E. Gain scheduling}

Some non linearities of the system are due to the nonlinearity of the lift and drag polars of the blade airfoils. Depending on the free wind speed, the airfoils operates in different regions of their polar curves. Those non-linearities are minimised by using gain scheduling on the average free wind speed inflow $V$. The model matrices $\mathbb{A}, \mathbb{B}, \mathbb{C}, \mathbb{C}_{m}$ and $\mathbb{G}$, and therefore $\Phi, \Phi_{\breve{y}}, \Phi_{\breve{u}}, \Gamma, \Gamma_{d}, \Gamma_{\breve{y}}, \Gamma_{\breve{u}}$ and $K$ all depend on $V$. They are calculated offline at several values of $V$, and they are interpolated online depending on the free wind speed.
Defining the mean free wind speed $V$ is tricky because of the spatial and time turbulence of the wind. Ostergaard et al. designed a wind speed estimator based on the rotor speed, the aerodynamic torque and the pitch angle [17]. The estimated effective wind speed is meant to be used for gain scheduling of their controllers. Instead, the mean free wind speed $V$ seen by the V27 turbine is estimated from a lookup table function of the pitch angle and the flap blade root moment of the 3 blades.

Low pass filters ensures that the estimated effective wind speed is a smooth function of time.

\section{RESULTS}

\section{A. Simulation code}

Numerical analysis is carried out with the aeroelastic code Flex5, developed by DTU Mechanical Engineering. It is a state-of-the art Blade Element Momentum code based on the modal approach. Both blades and tower are flexible, modeled by as many mode shapes as required. It has all the usual engineering models used in the wind turbine simulation tools, such as Prandtl-Glauert tip correction, dynamic wake model, turbulent wind, oblique inflow model etc. The dynamic stall model originally coded in Flex 5 works only for rigid blade sections [18]. The implementation of the trailing edge flaps aerodynamics in Flex 5 is based on the model written by Andersen et al. [14]. Flex5 has a fixed-step solver; a frequency of $50 \mathrm{~Hz}$ is used in those simulations.

Notice that the simulation model Flex 5 differs significantly from the design model (2). The simulation model takes into account the induced velocities lags, the aerodynamic lags and the elasticity of all the turbine components. The blade is no longer modeled by its first mode only, but by its three first modes. The pitch is actuated in order to regulate the produced power, and the rotor speed is no longer strictly constant. The Flex 5 code has been used for a long time, and its results match reasonably the measurements made on real turbines.

The open source code qpOASES [19][20] is used to solve the quadratic program, real-time, at $50 \mathrm{~Hz}$.

\section{B. Blade root flap moment spectral density}

Fig. 3 shows the typical spectral density of the flapwise blade root moment when the turbine is in normal production. The 1P frequency corresponds to one event per rotor revolution, the $2 \mathrm{P}$ and $3 \mathrm{P}$ frequencies are the double and the triple of the $1 \mathrm{P}$ frequency. Loads at those frequencies are consequences of events such as tower shadow, wind shear or yaw misalignment. Loads can also be seen at the first flapwise and the first edgewise eigenfrequencies of the blade. Those modes are usually well damped, either by the structural damping of the blade or by the aerodynamic damping.

Fatigue damage of the blade root depends on the number of load cycles the blade is going through, but also on the amplitude of those cycles. Loads at frequencies $1 \mathrm{P}, 2 \mathrm{P}$ and $3 \mathrm{P}$ are the most important loads regarding fatigue damage of the blade. 


\section{Frequency weighted control of the blade root moment}

The model predictive control described in this paper is designed in order to alleviate loads at given frequencies. This is done by tuning the filter on the outputs (7). A Butterworth bandpass filter of low order is used to decrease the loads at frequencies between the cut-in and the cut-out frequencies. The purpose of the controller is not to reduce the mean blade root flap moment, so the steady-state gain of the filter has to be 0 .

In a first time, 3 bandpass filters are designed so that the controllers MPC 1, MPC 2 and MPC 3 alleviate respectively the 1P, $2 \mathrm{P}$ and $3 \mathrm{P}$ loads. Simulations are run with Flex 5 with those 3 controllers, with exactly the same wind conditions. Fig. 3 shows the spectral densities of the blade root flap moment and of one of the 3 TEF angle. The 3 controllers manage to reduce the loads within the frequency range they have been designed for, MPC 1 being the best to reduce the 1P loads, MPC 2 the $2 \mathrm{P}$ loads and MPC 3 the $3 \mathrm{P}$ loads. Each of those 3 controllers reduce loads at frequencies between $1 \mathrm{P}$ and 3P. But, on the other hand, they increase the amplitude of the loads at frequencies higher than 3P: those high frequency, high amplitude loads damage a lot the materials. This wrong behavior of the controller is a consequence of a plant-model mismatch. The model does not include a dynamic model of the aero forces around the airfoil. At high actuation frequencies, the time lag between the TEF position and the change in lift coefficient can no longer be neglected, and the fact that it is not included in the design model of the MPC may end up in those extra loads.

This mismatch can be minimised by fine tuning the Kalman filter, and increasing the variance on the measurement noise $\nu$. Two new controllers, MPC 4 and MPC 5, are designed in order to alleviate the loads at frequencies between $1 \mathrm{P}$ and 3P. The Kalman filter of MPC 4 is the same as the one used in MPC 1 to MPC 3. In MPC 5, the Kalman filter is tuned in order to avoid the extra loads at frequencies higher than 3P. Fig. 4 shows that MPC 5 is as good as MPC 4 to alleviate loads at frequencies between $1 \mathrm{P}$ and $3 \mathrm{P}$, and, at the same time, it does no longer increase the loads at frequencies close to $4 \mathrm{P}$.

A last improvement of the MPC consists in decreasing the TEF activity at high frequencies, where they do not help reducing the blade root loads, but where they wear out the actuator systems. The filter on the inputs (8) is used for this purpose: a Butterworth high pass filter is designed in order to emphasise the cost on the high frequency trailing edge flaps actuation. MPC 6 is similar to MPC 5, but with this extra cost on the high pass filtered inputs. Figure 5 shows the results of simulations made with these controllers: the blade root loads with controllers MPC 5 and MPC 6 are similar over the whole spectrum, but actuators controlled by MPC 6 have a much lower activity at high frequencies. The damage of the blade root is unchanged, while the fatigue damage of the actuator system is decreased.
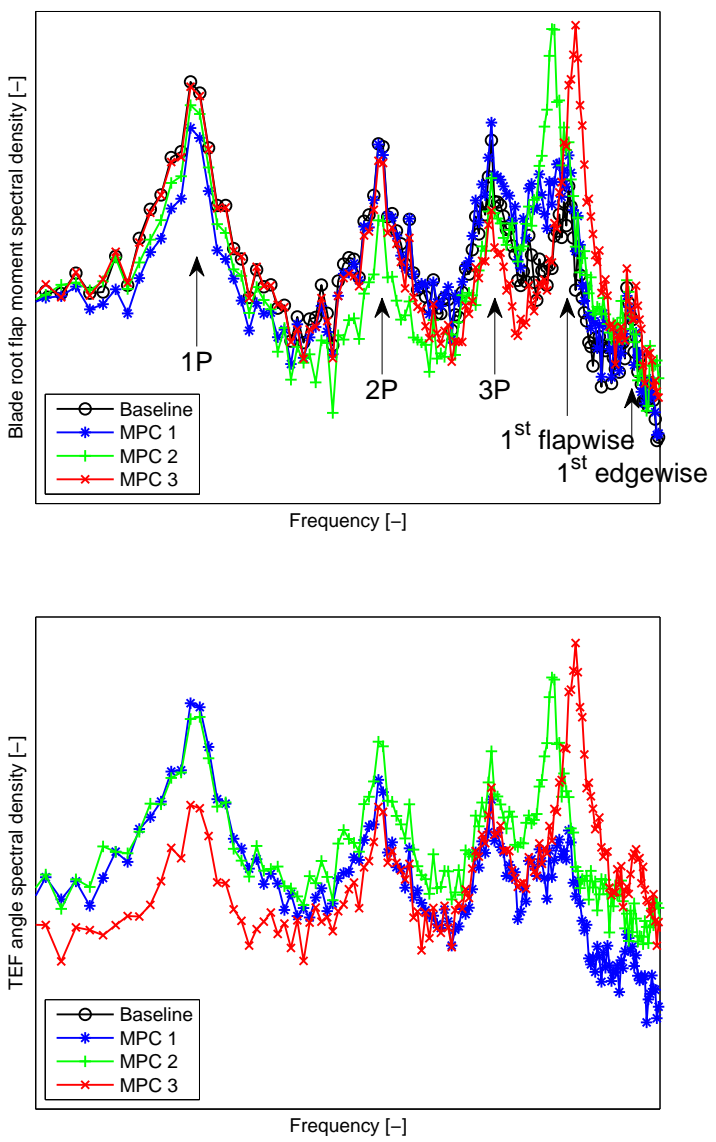

Fig. 3. Blade root flap moment (top) and trailing edge flaps angle (bottom) spectral density when TEF are not actuated (baseline test) and when controllers MPC 1, MPC 2 or MPC 3 are used to control the TEF. Those controllers aim at alleviating the blade root flap moment at respectively the $1 \mathrm{P}, 2 \mathrm{P}$ and $3 \mathrm{P}$ frequencies.

\section{CONCLUSION}

A model predictive control has been designed in order to alleviate blade root loads with emphasize on given load frequencies. This controller, while used with the Flex5 simulation code, shows its ability to focus on fixed frequencies. The tuning of the Kalman filter and of the inputs filter are essential in order to reach a good trade off between blade damage and actuator damage. Robustness and stability will have to be tested before testing on the V27 turbine at Ris $\varnothing$ DTU, National Laboratories for Sustainable Energy.

\section{REFERENCES}

[1] T. J. Larsen, H. A. Madsen, and K. Thomsen, "Active load reduction using individual pitch, based on local blade flow measurements," Wind Energy, vol. 8, no. 1, pp. 67-80, 2005.

[2] E. A. Bossanyi, "Individual blade pitch control for load reduction," Wind Energy, vol. 6, pp. 229-244, 2003.

[3] T. Barlas and G. van Kuik, "Review of state of the art in smart rotor control research for wind turbines." Prog Aerospace Sci, 2009.

[4] T. Buhl, M. Gaunaa, and C. Bak, "Potential load reduction using airfoils with variable trailing edge geometry," Journal of Solar Energy Engineering, vol. 127, pp. 503-516, November 2005. 

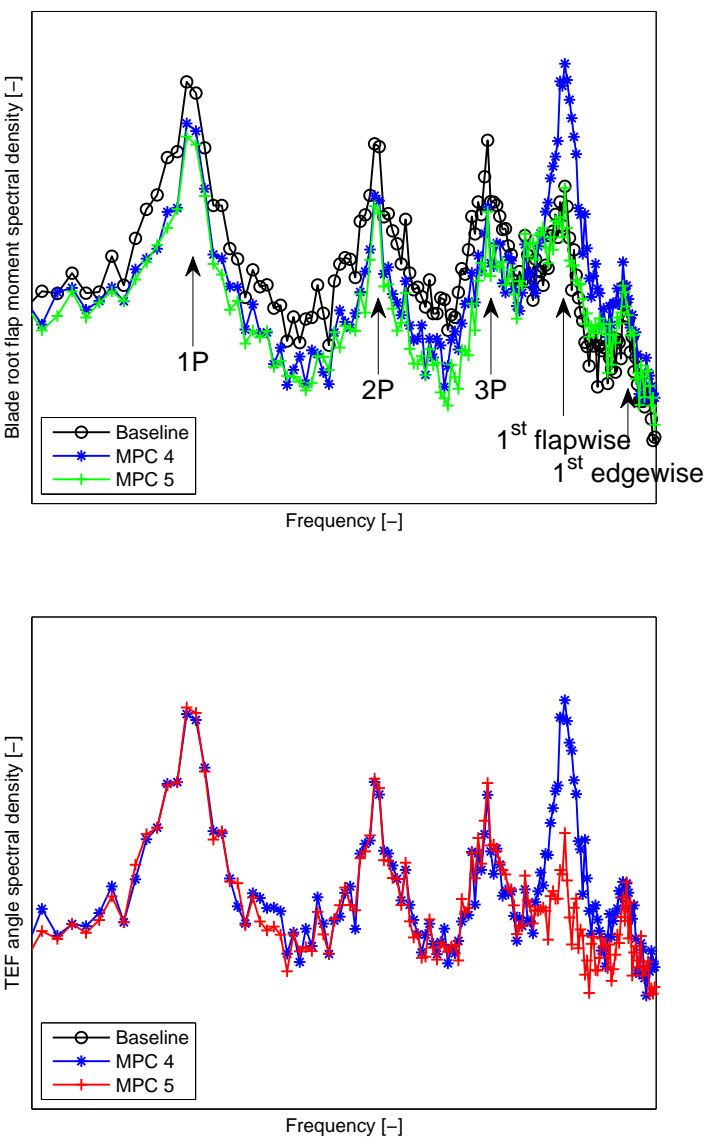

Fig. 4. Blade root flap moment (top) and trailing edge flap angle (bottom) spectral density when TEF are not actuated (baseline test) and when controllers MPC 4 or MPC 5 are used. Those controllers aim at alleviating the blade root flap moment at frequencies up to 3P. The Kalman filter used in the controller MPC 5 is tuned in order to avoid extra loads at frequencies higher than $4 \mathrm{P}$

[5] P. B. Andersen, M. Gaunaa, C. Bak, and T. Buhl, "Deformable trailing edge flaps for modern megawatt wind turbine controllers using strain gauge sensors," Wind Energy, vol. 13, no. 2, pp. 193-206, 2010.

[6] C. Bak, M. Gaunaa, P. B. Andersen, T. Buhl, P. Hansen, and K. Clemmensen, "Wind tunnel test on airfoil Ris $\varnothing-\mathrm{b} 1-18$ with an active trailing edge flap," Wind Energy, vol. 13, no. 2-3, pp. 207-219, 2010.

[7] J. W. van Wingerden, A. W. Hulskamp, T. Barlas, B. Marrant, G. A. M. van Kuik, D.-P. Molenaar, and M. Verhaegen, "On the proof of concept of a 'smart' wind turbine rotor blade for load alleviation," Wind Energy, vol. 11, pp. 265-280, 2008.

[8] J. W. van Wingerden, A. Hulskamp, T. Barlas, I. Houtzager, H. Bersee, G. van Kuik, and M. Verhaegen, "Two-degree-of-freedom active vibration control of a prototyped smart rotor," IEEE Transactions on Control Systems Technology, vol. 19, no. 2, pp. 284-296, 2011.

[9] D. Castaignet, J. J. Wedel-Heinen, T. Kim, T. Buhl, and N. K. Poulsen, "Results from the first full scale wind turbine equipped with trailing edge flaps." Chicago, Illinois: 28th AIAA Applied Aerodynamics Conference, June 2010

[10] M. Lackner and G. van Kuik, "A comparison of smart rotor control approaches using trailing edge flaps and individual pitch control," Wind Energy, vol. 13, pp. 117-134, 2010.

[11] J. K. Rice and M. Verhaegen, "Robust and distributed control of a smart blade," Wind Energy, vol. 1, pp. 103-116, 2010.

[12] D. G. Wilson, D. E. Berg, M. F. Barone, J. C. Berg, B. R. Resor, and D. W. Lobitz, "Active aerodynamic blade control design for load
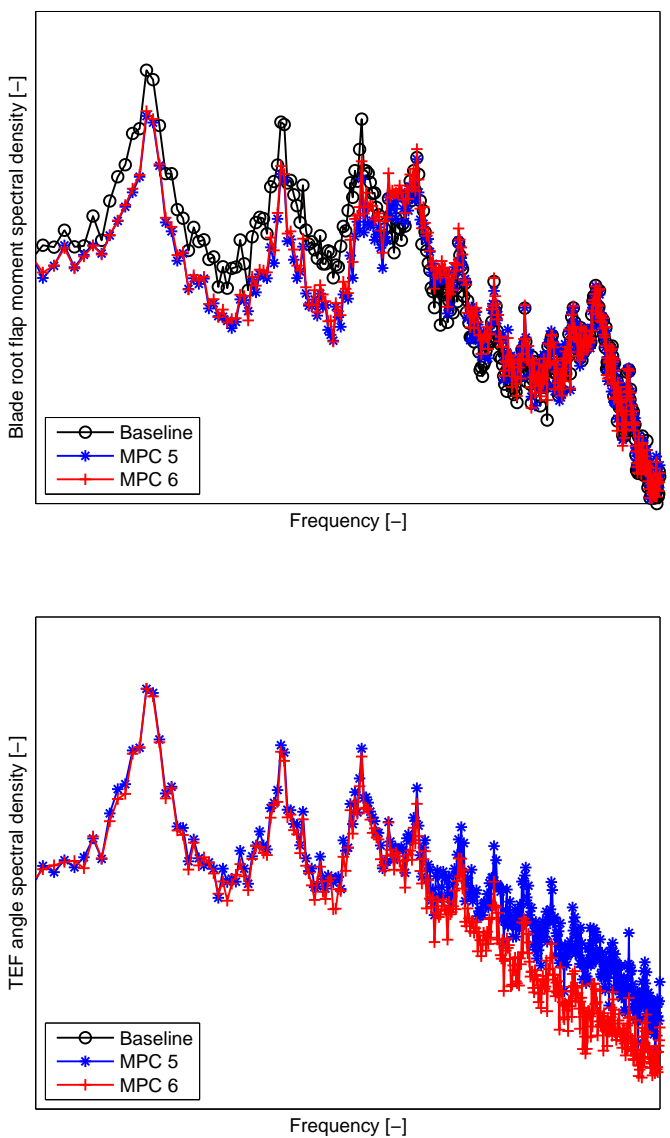

Fig. 5. Blade root flap moment (top) and trailing edge flap angle (bottom) spectral density when TEF are not actuated (baseline test) and when controllers MPC 5 or MPC 6 are used. Those controllers aim at alleviating the blade root flap moment at frequencies up to 3P. A cost on the high pass filtered inputs is added in MPC 6 , resulting in a decrease of the actuator activity at high frequencies, without decreasing the controller efficiency.

reduction on large wind turbines." Marseille, France: European Wind Energy Conference \& Exhibition, March 2009.

[13] M. O. L. Hansen, Aerodynamics of Wind Turbines. Earthscan, 2008.

[14] P. B. Andersen, M. Gaunaa, C. Bak, and M. H. Hansen, "A dynamic stall model for airfoils with deformable trailing edges," Wind Energy, vol. 12 , no. 8, pp. 734-751, 2009.

[15] K. Hammerum, P. Brath, and N. K. Poulsen, "A fatigue approach to wind turbine control," Journal of Physics: Conference Series, vol. 75, no. 1, 2007.

[16] G. Pannocchia and J. B. Rawlings, "Disturbance models for offset-free model-predictive control," AIChE Journal, vol. 49, no. 2, pp. 426-437, 2003.

[17] K. Z. Ostergaard, P. Brath, and J. Stoustrup, "Estimation of effective wind speed," Journal of Physics: Conference Series, vol. 75, no. 1, 2007.

[18] S. Øye, "Dynamic stall simulated as time lag of separation." Thessaloniki, Greece: European Wind Energy Conference EWEC, October 1994.

[19] qpOASES homepage. [Online]. Available: http://www.kuleuven.be/optec/software/qpOASES

[20] H. Ferreau, H. Bock, and M. Diehl, "An online active set strategy to overcome the limitations of explicit mpc," International Journal of Robust and Nonlinear Control, vol. 18, no. 8, pp. 816-830, 2008. 


\section{Paper B}

Frequency-Weighted Model Predictive Control of Trailing Edge Flaps on a Wind Turbine Blade

Paper submitted at the IEEE special issue "To tame the wind: advanced control applications in wind energy".

Personal use of this material is permitted. However, permission to reprint/republish this material for advertising or promotional purposes or for creating new collective works for resale or redistribution to servers or lists or to reuse any copyrighted component of this work in other works must be obtained from the IEEE.

This work has been submitted to the IEEE for possible publication. Copyright may be transferred without notice, after which this version will be superseded. 


\title{
Frequency-Weighted Model Predictive Control of Trailing Edge Flaps on a Wind Turbine Blade
}

\author{
Damien Castaignet, Ian Couchman, Niels K. Poulsen, Thomas Buhl, and Jens Jakob Wedel-Heinen
}

\begin{abstract}
This paper presents the load reduction achieved with trailing edges flaps during a full-scale test on a Vestas V27 wind turbine. The trailing edge flap controller is a frequency-weighted linear model predictive control (MPC) where the quadratic cost consists in costs on the zero-phase filtered flapwise blade root moment and trailing edge flap deflection. Consistent load reduction is achieved during the full-scale test. An average of $13.8 \%$ flapwise blade root fatigue load reduction is achieved during a 38 minute test with a $70 \mathrm{~cm}$ long hinged trailing edge flap on the $13 \mathrm{~m}$ long V27 blade.
\end{abstract}

Index Terms-Model predictive control, load alleviation, trailing edge flaps, wind energy.

\section{INTRODUCTION}

Wind turbine rotor size has increased significantly over the last years, with most of the major wind turbine manufacturers now marketing turbines with rotor diameters larger than 100 $\mathrm{m}$. Increasing the blade span is an effective way to increase the power production of a turbine, but it comes at the cost of increased loads on all the turbine components. The fatigue and extreme loads that a wind turbine undergoes during its 20 year lifetime drive the design of the main components; e.g. the blades, the tower, the drive train and the foundation [1]. The requirement to sustain higher loads leads typically to heavier and more expensive components. With the energy prices from a wind farm being dominated by capital expenditure, the search for load alleviating technologies is well motivated.

The forces and moments a wind turbine experiences originate from a number of sources: wind conditions, such as wind turbulence or wind shear, tower shadow, resonance of wind turbine modes, yaw misalignment of the turbine, emergency shutdowns, grid loss etc [2]. Some modern wind turbines use cyclic pitch or individual pitch in order to alleviate some of the loads. Cyclic pitch, a technology developed by the rotorcraft community, involves pitching the three blades with a fixed angle phase shift (in the case of a 3 bladed wind turbine, 120 degrees). It is used to alleviate tilt and yaw loads on the rotor. The natural extension to cyclic pitch is individual pitch control (IPC) where each blade can pitch independently of the two other blades. IPC is the one of the most advanced active

This work was partially funded by the Danish National Advanced Technology Foundation (Højteknologifonden), as part of the ATEF (Adaptive Trailing Edge Flaps) project.

D. Castaignet, I. Couchman and J.-J. Wedel-Heinen are with Vestas Wind Systems A/S, Global Research \& Innovation. e-mail: \{daca, iacou, jjwh\} @ vestas.com

N. K. Poulsen is with the Technical University of Denmark, DTU Informatics. e-mail: nkp@imm.dtu.dk

T. Buhl is with the Wind Energy department of Ris $\varnothing$ DTU, National Laboratories for Sustainable Energy. e-mail: thbu@risoe.dtu.dk control to alleviate loads in todays turbines [3], [4], [5], [6], [7] but its performance is limited by the relatively slow pitch actuators and by the necessity to limit pitch actuation in order to preserve the actuators and the blade bearings. Some passive load controls, like prebent blades, swept blades [8], [9] or bending/twist-coupled blades [10], [11], show also promising load reductions.

In order to reduce even further and in a more efficient way the loads on the rotor, several concepts of "smart" rotors have been investigated. A "smart" rotor consists in distributed sensors (accelerometers, strain gauges, Pitot tubes, pressure tabs etc.) and actuators (trailing edge flaps, microtabs, boundary layer suction or blowing jets, plasma actuators etc.) along the blades. A detailed overview of different "smart" rotor concepts is given in [12].

Trailing edge flaps on turbine blades have been investigated for several years. CFD simulations [13] and 2D [14], [15] and 3D [16], [17] aeroelastic simulations confirmed the high potential of trailing edge flaps to reduce flapwise blade root fatigue loads. Wind tunnel tests on a blade section [18], [19], [20] as well as on a scaled turbine [21] corroborated the ability of the trailing edge flaps to reduce loads. At last, a full scale test was carried out on a Vestas V27 turbine at Ris $\varnothing$ DTU. Only open loop controls were tested at that time [22].

Several control strategies have been investigated as well. Lackner et al. designed a PID Individual Flap Control based on the Individual Pitch Control scheme, using the Coleman transformation to make the system linear time invariant [23]. Van Wingerden et al. used subspace system identification to fine tune the PD controller used in their wind tunnel test [19], and developed a feedback controller based on $\mathcal{H}_{\infty}$-loop shaping combined with a fixed-structure feedforward control which they succesfuly tested on the scaled turbine in a wind tunnel [21]. Rice et al. focused on a robust and distributed control in order to ensure stability of the controller despite non linearities and model mismatch [24]. At last, Wilson et al. designed PD feedback controllers based on tip deflection or tip deflection rate, and showed a decrease in the standard deviation of the root flap bending moments [25].

The performance of all smart rotor concepts is restricted by constraints on the actuators; for example in the case of trailing edge flaps, limits on the rate at which the flaps can be moved and maximum flap deflection in each direction. The presence of these constraints makes model predictive control a suitable candidate. In the model predictive control paradigm, the current control input is set by solving a finite horizon, open-loop optimal control problem, with the current state providing the initial condition for the model of the plant, 


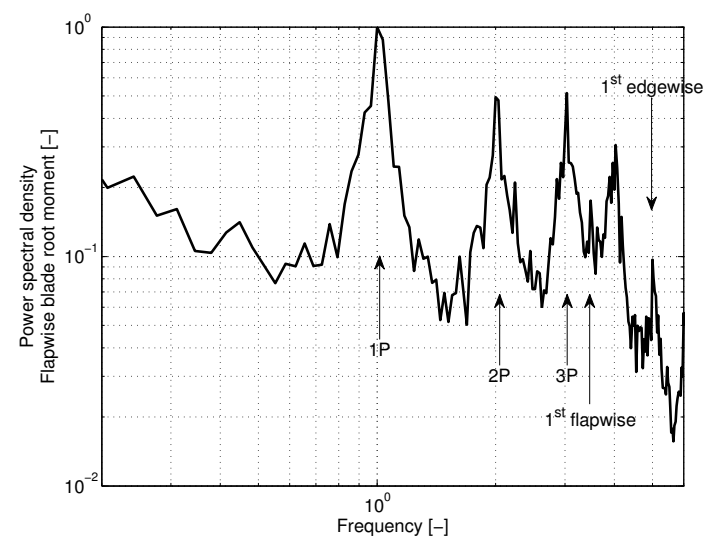

Fig. 1. Power spectral density of the flapwise blade root bending moment of the V27. It shows peaks at the 1P, $2 \mathrm{P}$ and $3 \mathrm{P}$ frequencies, and at the blade first flapwise and edgewise eigenfrequencies.

see [26]. This methodology allows constraints to be explicitly accounted for without surrendering performance.

The main contribution of this paper is the design and implementation and test of a model predictive controller for a full scale site test on the aforementioned V27.

\section{Problem Statement}

\section{A. Process}

This paper focuses on normal production load cases, where the most important contribution to the wind induced fatigue loads in the rotor are the consequences of azimuth-dependent wind conditions, like wind shear, wind slope, yaw error, tower shadow, local turbulence etc. The frequency of those azimuthdependent loads are function of the rotor speed. Their spectral density clearly shows the first harmonics of the rotor speed. Those harmonics are typically noted $1 \mathrm{P}, 2 \mathrm{P}, 3 \mathrm{P}$, etc. where the $1 \mathrm{P}$ frequency corresponds to one event per rotor revolution, and the $2 \mathrm{P}$ and $3 \mathrm{P}$ frequencies are twice and three times larger (Fig. 1).

Besides the 1P, 2P and 3P loads, the first blade eigenfrequencies are seen in the PSD of the blade root moment. They are the consequence of the excitation of an eigenmode of the turbine which is dominated by flapwise or edgewise blade vibrations. In Fig. 1 both the first flapwise and edgewise eigenfrequencies of the blade are observed.

Those varying loads are responsible for the fatigue damage of the turbine components. All the turbine components are designed in order to last for a given amount of time: they have to resist to both the fatigue and extreme loads that the turbine will face during this time. Reducing the fatigue can lead to a cheaper design of the wind turbine, and thus a cost of energy reduction.

\section{B. Actuation and sensing mechanisms}

Trailing edge flaps are local active flow control device. They modify the aerodynamic properties (lift, drag and moment coefficients) of the airfoil by changing its camber. By modulating the local forces at some blade sections, it is possible to compensate for the loads variations due to the varying local wind speeds seen by the rotating blade. Trailing edge flaps are usually mounted towards the tip of the blade, where they have the largest impact.

The sensors available for the purpose of the controller are, in this demonstrator, the blade root strain gage measuring the flapwise blade root moment, the Pitot tubes and the pitch position sensor. The sensors in the nacelle, the tower or on the met mast are not available to the controller in this demonstrator.

\section{Controller objectives}

The most direct purpose of trailing edge flaps is to reduce blade root flapwise fatigue loads. The reduction in blade root fatigue loads usually leads to reduction of fatigue and extreme loads in all the major components of the turbine [17].

The fatigue damage of the material is estimated by the equivalent stress range $\sigma^{e q}$ : the fatigue damage of a material going throught $N_{i}$ cycles of s stress range $\sigma_{i}$ is equivalent to the fatigue damage of the same material going thought $N^{e q}$ cycles with a stress range $\sigma^{e q}$. An approximation of the Wöhler curve is used to calculate $\sigma^{e q}$ :

$$
\sigma^{e q}=\sum_{i} \sigma_{i}\left(\frac{N_{i}}{N^{e q}}\right)^{1 / m} .
$$

$m$ is a material constant between 8 and 12 for glass fibres.

Such a function is not quadratic, and a cost function which would consist in minimising $\sigma^{e q}$ for the flapwise blade root moment could not be solved by a quadratic program. But it is noticed that the flapwise blade root fatigue loads are due mainly to the $1 \mathrm{P}$ loads, and secondarily to the $2 \mathrm{P}$ and $3 \mathrm{P}$ loads. The objective of the controller is thus to reduce the amplitude of the 1P, 2P and 3P loads. Loads with frequencies lower than the $1 \mathrm{P}$ frequency should not be targeted; they are due to the free wind speed fluctuations and reducing them would result in important power loss. Besides, they do no contribute significantly to the blade fatigue loads. Loads with frequencies higher than the $3 \mathrm{P}$ frequency have also a negligible impact on fatigue loads due to their low amplitude. They should not be targeted as they would add unnecessary actuation of the trailing edge flaps. Moreover, the design model is not accurate for such frequencies and an attempt of the controller to reduce the amplitude of loads with such high frequencies may make actually lead to an increase of those loads.

\section{DESCRIPTION OF THE EXPERIMENT}

An already existing V27 turbine has been modified for the purpose of the project. Trailing edge flaps have been implemented on one of the blades in order to demonstrate the feasibility of the "smart" rotor and the performance of the model predictive control.

\section{A. Wind turbine}

The Vestas V27 turbine is an horizontal axis wind turbine, with a nominal power of $225 \mathrm{~kW}$, and 13 meter long blades. Even if it was designed in the eighties, it is, like most of 
today's turbines, a pitch controlled turbine. The blades are pitched in or out of the wind in order to regulate the power production of the rotor. The flow remains most of the time attached to the airfoil, which is necessary for fully benefiting from the trailing edge flaps. The V27 operated at two different rotor speeds: around $32 \mathrm{rpm}$ for wind speeds below 4 to 5 $\mathrm{m} . \mathrm{s}^{-1}$, and around $43 \mathrm{rpm}$ for higher wind speeds.

A meteorological mast is located $70 \mathrm{~m}$ west of the Vestas V27. It measures the wind speeds and directions at different heights. The test site is located on the seaside: the sea is west of the wind turbine, some fields are east of the turbine, some buildings and a small village are north and south of the turbine. This gives very different wind conditions depending on the wind direction. A bigger turbine is located $100 \mathrm{~m}$ south of the Vestas V27: the V27 can also be tested in wake conditions.

The V27 turbine used for the Adaptive Trailing Edge Flap (ATEF) project is located at Risø DTU, National Laboratory for Sustainable Energy, Roskilde, Denmark.

\section{B. Actuators and sensors}

As it has been stated by van Wingerden and al [21], "the most challenging part in the design of the "smart" rotor is the design of the actuator". One of the original blades of the V27 has been modified and is now equipped with 3 independent trailing edge flaps (Fig. 2). The total length of the flaps is $15 \%$ of the blade span.

Extra sensors have also been mounted on the turbine for the purpose of the experiment. The sensors available for the trailing edge flaps controller include three Pitot tubes located at the leading edge of the blade, in the middle of each trailing edge flap, pressure tabs at the leading edge of the blade, in the middle of each trailing edge flap, strain gages at the blade root, flapwise and edgewise, accelerometers in the blade, pitch position. The Pitot tubes are 5-hole probes, which are calibrated in order to output the local angle of attack and relative wind speed. Other sensors are available for logging only: accelerometers in the nacelle, strain gages at the tower bottom, power production, nacelle anenometer etc. They are used for monitoring the turbine and investigating the effect of the flaps on the whole turbine.

The configuration of actuators and sensors used in this paper matches the experimental blade available within the ATEF project.

\section{Real-Time environment}

The V27 pitch controller has not been modified. The trailing edge flaps controller described in this paper runs independently of the pitch controller, on a separate hardware.

The trailing edge flaps controller runs on a NI cRio 9024, which is a Real-Time controller from National Instruments running at $800 \mathrm{MHz}$ and having $512 \mathrm{MB}$ DRAM. The NI cRio 9024 is phisically located in the spinner of the turbine. A graphical interface runs on a separate computer located in a control room.

Logging from the sensors is made with several NI cDAQ 9172, sitting in the spinner, the nacelle and next to the met mast.

\section{DESIGN MODEL}

Considering that only one blade is equipped with trailing edge flaps, and that only the blade sensors are available for the controller, it has been decided to limit the design model to a single rotating blade. The tower and the shaft flexibilities are not modeled. First, a non-linear model is derived in order to calculate the steady-point of the system, for different free wind speeds. It is then simplified and linearised analytically.

\section{A. Non-linear model}

The non-linear model of the rotating blade includes all the main models used in the aero-elastic code Flex developped at DTU Mechanical Engineering: dynamic stall and wake models, tip loss, centrifugal loads etc. [27]

1) Structural model: Generalised coordinates and blade eigenmodes are used to model the structure of the blade. It is assumed that the deflection of a blade section at radius $r$, $r \in[0 ; L]$, at time $t$ can be decoupled into a time dependent function (the generalised coordinates) and a spanwise dependent function (the blade eigenmodes). $L$ is the blade length. The more eigenmodes are taken into account, the more accurate the model is. But the V27 blade is so stiff that most of the blade deflection is due to the first blade eigenmode only. The next eigenmodes have high eigenfrequencies which are hardly excited.

The flapwise deflection $u_{z}(r, t)$ and the edgewise deflection $u_{y}(r, t)$ of blade section $r$ at time $t$ can thus be simplified into:

$$
u_{z}(r, t)=g(t) u_{z}^{1}(r), \quad u_{y}(r, t)=g(t) u_{y}^{1}(r)
$$

$u_{z}^{1}(r)$ and $u_{y}^{1}(r)$ are respectively the flapwise and edgewise deflection of the blade section $r$ under the first mode shape. $g(t)$ is the generalised coordinate associated to the first mode shape.

The torsion of the blade is not part of this model. This is not an issue for the V27 blade due to its high torsion stiffness, but may be an issue for larger blades.

2) Aerodynamic model: The aerodynamic forces on an airfoil with a trailing edge flap depend on the local relative wind speed $\mathbf{V}_{\mathbf{r}}(r, t)$, the local angle of attack $\alpha(r, t)$ and the trailing edge flap angle $\beta(r, t)$. The relative wind speed $\mathbf{V}_{\mathbf{r}}(r, t)$ seen by the airfoil is the sum of the free wind speed

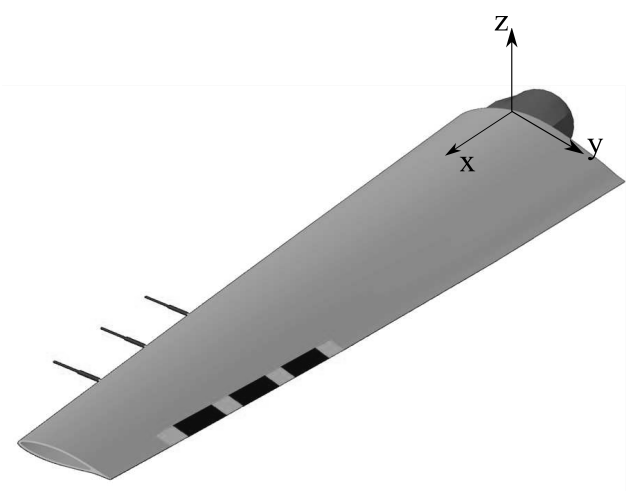

Fig. 2. Illustration of the Vestas V27 blade equipped with trailing edge flaps, passive transient pieces and Pitot tubes. 
$\mathbf{V}_{\mathbf{0}}(r, t)$, the induced velocities $\mathbf{W}(r, t)$ and the opposite of the airfoil speed $-\mathbf{V}_{\mathbf{b}}(r, t)$ :

$$
\mathbf{V}_{\mathbf{r}}(r, t):=\mathbf{V}_{\mathbf{0}}(r, t)+\mathbf{W}(r, t)-\mathbf{V}_{\mathbf{b}}(r, t) .
$$

$:=$ denotes the assignment symbol. The induced velocities are induced by the spinning rotor, which slows down the wind when approaching the rotor. They are mainly dependent on the thrust generated on the rotor by the aerodynamic forces. They are derived from the unsteady Blade Element Momentum [27].

At last, a dynamic stall model is used in order to derive the dynamic lift and drag coefficients, $C_{L}$ and $C_{D}$, of the airfoil with a given angle of attack $\alpha$, trailing edge flap angle $\beta$ and relative wind speed $\mathbf{V}_{\mathbf{r}}$. Andersen and al. derived such a dynamic stall model for an airfoil with a trailing edge flap [28]. The flapwise and edgewise aerodynamic loads, $p_{z}^{\text {aero }}(r, t)$ and $p_{y}^{\text {aero }}(r, t)$, are derived from the lift and drag coefficients:

$$
\begin{aligned}
p_{z}^{\text {aero }}(r, t, \beta):= & \frac{1}{2} \rho\left|\mathbf{V}_{r}\right|^{2} C(r)\left(C_{L}(r, t, \beta) \cos (\alpha)\right. \\
& \left.+C_{D}(r, t, \beta) \sin (\alpha)\right) \\
p_{y}^{\text {aero }}(r, t, \beta):= & \frac{1}{2} \rho\left|\mathbf{V}_{r}\right|^{2} C(r)\left(-C_{L}(r, t, \beta) \sin (\alpha)\right. \\
& \left.+C_{D}(r, t, \beta) \cos (\alpha)\right)
\end{aligned}
$$

where $\rho$ is the air density, and $C(r)$ the airfoil chord length at the blade section $r$.

3) Differential equation: Newton's second law applies to the generalised coordinate $g(t)$ :

$$
M_{g} \ddot{g}+C_{g} \dot{g}+K_{g} g=F_{g}(\beta) .
$$

$M_{g}, C_{g}$ and $K_{g}$ are respectively the generalised mass, damping and stiffness associated to the first blade eigenmode. They depend on the blade structure properties only.

The generalised force $F_{g}$ is the work done by the external loads (aerodynamic, centrifugal, inertia and gravity loads) on the first blade eigenmode:

$$
F_{g}(t)=\int p_{z}(r, t) u_{z}(r, t) \mathrm{d} r+\int p_{y}(r, t) u_{y}(r, t) \mathrm{d} r .
$$

$p_{y}(r, t)$ and $p_{z}(r, t)$ are the external loads, respectively flapwise and edgewise, acting on the blade section at a radius $r$.

The differential equations relative to the dynamic stall model and the induced velocities can be found in [28] and [27].

4) Blade root moment: The flapwise blade root moment, measured by a strain gage, is $M_{z}:=E I \frac{\partial^{2} u_{z}(x, t)}{\partial x^{2}}$, where $E$ is the blade root's modulus of elasticity, flapwise, and $I$ its moment of inertia.

Note that actuators are modeled as perfect actuators: the demand and the response of the trailing edge flap are identical.

\section{B. Linear model}

Several simplifications are made on the non-linear model previously described before it is linearised:

- The rotor speed is considered constant. The Vestas V27 turbine generator has 2 fixed generator speeds: a low speed for low wind speeds, and a faster speed for higher wind speeds. The switch between the low and the high speeds happens at wind speeds of 4 to $5 \mathrm{~ms}^{-1}$. For the test, two different controllers are used depending on the rotor speed;

- Airfoil aerodynamics lags are also neglected. The Vestas V27 is a pitch controlled wind turbine. In normal production, the flow around the airfoils stays attached, and the airfoil aerodynamic lags are then negligible. The lift and drag coefficients $C_{L}(r, t, \beta)$ and $C_{D}(r, t, \beta)$ in (4b) and (4a) are thus steady-state values;

- Induced velocities, which have a slow dynamic, are considered constant;

- Gravity loads are neglected. In normal production, the pitch angle is small and gravity loads have thus an impact mainly on the edgewise blade loads; and

- The blade is equipped with three independent flaps. In the rest of the paper, the vector $\beta$ is the vector of the three trailing edge flap angles.

The governing nonlinear equations are expressed, after applying the previous simplifications, as:

$$
\dot{x}=f(x, u, d)
$$

where $x:=(g, \dot{g})$ is the vector of the wind turbine blade states, $u:=(\beta)$ is the vector of the control inputs and $d:=(\varphi)$ is the vector of the measured disturbances. The pitch position $\varphi$ is considered as a measured disturbance and not a control input because the pitch actuator is controlled by another actuator, and the trailing edge flaps controller can only have access to the pitch position.

The nonlinear equations (6) are linearised by using the first order term of Taylor expansion in the neighbourhood of the operating points $\left(x^{*}, u^{*}, d^{*}\right)$ which are solutions of $f\left(x^{*}, u^{*}, d^{*}\right)=0$. The operating points are function of the mean free wind speed $V$, and are calculated for different values of $V$ within the operating range, with a $2 \mathrm{~ms}^{-1}$ step.

The linearised generalised force for the first mode shape is then:

$$
\begin{gathered}
\bar{F}_{g}=\left.\frac{\partial F_{g}}{\partial g}\right|_{g=g^{*}}(\gamma) \bar{g}+\left.\frac{\partial F_{g}}{\partial \dot{g}}\right|_{\dot{g}=0}(\gamma) \dot{\bar{g}}+\left.\frac{\partial F_{g}}{\partial \varphi}\right|_{\varphi=\varphi^{*}}(\gamma) \bar{\varphi} \\
+\left.\frac{\partial F_{g}}{\partial \beta}\right|_{\beta=\beta^{*}}(\gamma) \bar{\beta}
\end{gathered}
$$

where variables with a are the difference between a variable and its steady-state value: $\overline{F_{g}}=F_{g}-F_{g}^{*}$ and $\gamma$ is an estimate of the mean free wind speed $V$.

The differential equation (5) is thus

$$
\begin{aligned}
M_{g} \ddot{\bar{g}}+C_{g} \dot{\bar{g}}+K_{g} \bar{g}= & \left.\frac{\partial F_{g}}{\partial g}\right|_{g=g^{*}}(\gamma) \bar{g}+\left.\frac{\partial F_{g}}{\partial \dot{g}}\right|_{\dot{g}=0}(\gamma) \dot{\bar{g}} \\
& +\left.\frac{\partial F_{g}}{\partial \varphi}\right|_{\varphi=\varphi^{*}}(\gamma) \bar{\varphi}+\left.\frac{\partial F_{g}}{\partial \beta}\right|_{\beta=\beta^{*}}(\gamma) \bar{\beta}
\end{aligned}
$$

and the blade root flap moment $\bar{M}_{f}=E I \frac{\partial^{2} u_{z}^{1}(x)}{\partial x^{2}} \bar{g}$.

The blade design model is, written as a state-space form:

$$
\begin{aligned}
\dot{\mathbf{x}}_{b} & =\mathbb{A}_{b}(\gamma) \mathbf{x}_{b}+\mathbb{B}_{b}(\gamma) \mathbf{u}+\mathbb{G}_{b}(\gamma) \mathbf{d} \\
\mathbf{z} & =\mathbb{C}_{b} \mathbf{x}_{b}, \mathbf{y}=\mathbb{C}_{b}^{m} \mathbf{x}_{b}
\end{aligned}
$$


where the state vector $\mathbf{x}_{b}:=(\bar{g}, \dot{\bar{g}})$, the input vector $\mathbf{u}:=\bar{\beta}$, the measured disturbance vector $\mathbf{d}:=(\bar{\varphi})$, the output vector $\mathbf{z}:=\left(\bar{M}_{f}\right)$, the measurement vector $\mathbf{y}:=\left(\bar{M}_{f}\right)$ and the state space matrices are

$$
\begin{aligned}
\mathbb{A}_{b}(\gamma) & :=\left[\begin{array}{cc}
0 & 1 \\
\mathbb{A}_{1}(\gamma) & \mathbb{A}_{2}(\gamma)
\end{array}\right] \\
\mathbb{B}_{b}(\gamma) & :=\left[\begin{array}{cc}
0 \\
M_{g}^{-1} & \left.\frac{\partial F_{g}}{\partial \beta}\right|_{\beta=\beta^{*}}(\gamma)
\end{array}\right] \\
\mathbb{G}_{b}(\gamma) & :=\left[\begin{array}{cc}
\left.M_{g}^{-1} \frac{\partial F_{g}}{\partial \varphi}\right|_{\varphi=\varphi^{*}}(\gamma)
\end{array}\right] \\
\mathbb{C}_{b} & :=\mathbb{C}_{b}^{m}=\left[\begin{array}{ll}
E I \frac{\partial^{2} u_{z}^{1}(x)}{\partial x^{2}} & 0
\end{array}\right]
\end{aligned}
$$

with

$$
\begin{aligned}
& \mathbb{A}_{1}(\gamma):=M_{g}^{-1}\left(\left.\frac{\partial F_{g}}{\partial g}\right|_{g=g^{*}}(\gamma)-K_{g}\right) \\
& \mathbb{A}_{2}(\gamma):=M_{g}^{-1}\left(\left.\frac{\partial F_{g}}{\partial \dot{g}}\right|_{\dot{g}=0}(\gamma)-C_{g}\right) .
\end{aligned}
$$

Fig. 3, 4 and 5 show a comparison of the following models:

- the Flex code, where the V27 turbine is modeled as a flexible turbine. This is the model used to simulate the turbine when testing the controller. Gravity is turned off in these simulations in order to make comparison easier;

- the Flex code, where most of the turbine degrees of freedom are stiff. Only the blade first mode is made flexible. This model is the closest to the design model developed in this section;

- the blade non linear model developed in section IV-A (Fig. 4 and 5 only); and

- the blade linear model developed in section IV-B.

Fig. 3 shows the Bode plots for the SISO system trailing edge flap angle to flapwise blade root moment, at a fixed rotor speed and pitch position. The Bode plot is derived at a fixed wind speed, below the rated wind speed. The Bode plots for the non-linear model are derived by actuating the trailing edge flaps at their maximum amplitude. They show a good agreement of the linear model with the simulation model for the frequencies of interest, between $0.5 \mathrm{P}$ and $4 \mathrm{P}$. The small drops around $1 \mathrm{P}$ and $5 \mathrm{P}$ are however not captured by the linear model. They are due to the fore-aft tower mode and the blade second eigenmode.

The flapwise blade root moment response to a discrete change in wind speed (Fig. 4) and in trailing edge flap angle (Fig. 5), both below rated power and above rated power, shows also a good agreement between the different models. The steady-state error that can be noticed between the Flex models and the non-linear and linear blade model is the consequence of the induced velocities.

\section{CONTROL SYSTEM DESIGN}

\section{A. Model Predictive Control}

The pitch controller of the V27 test turbine has not been modified for use with the flaps. It only aims at maximising the power production in normal production by pitching the
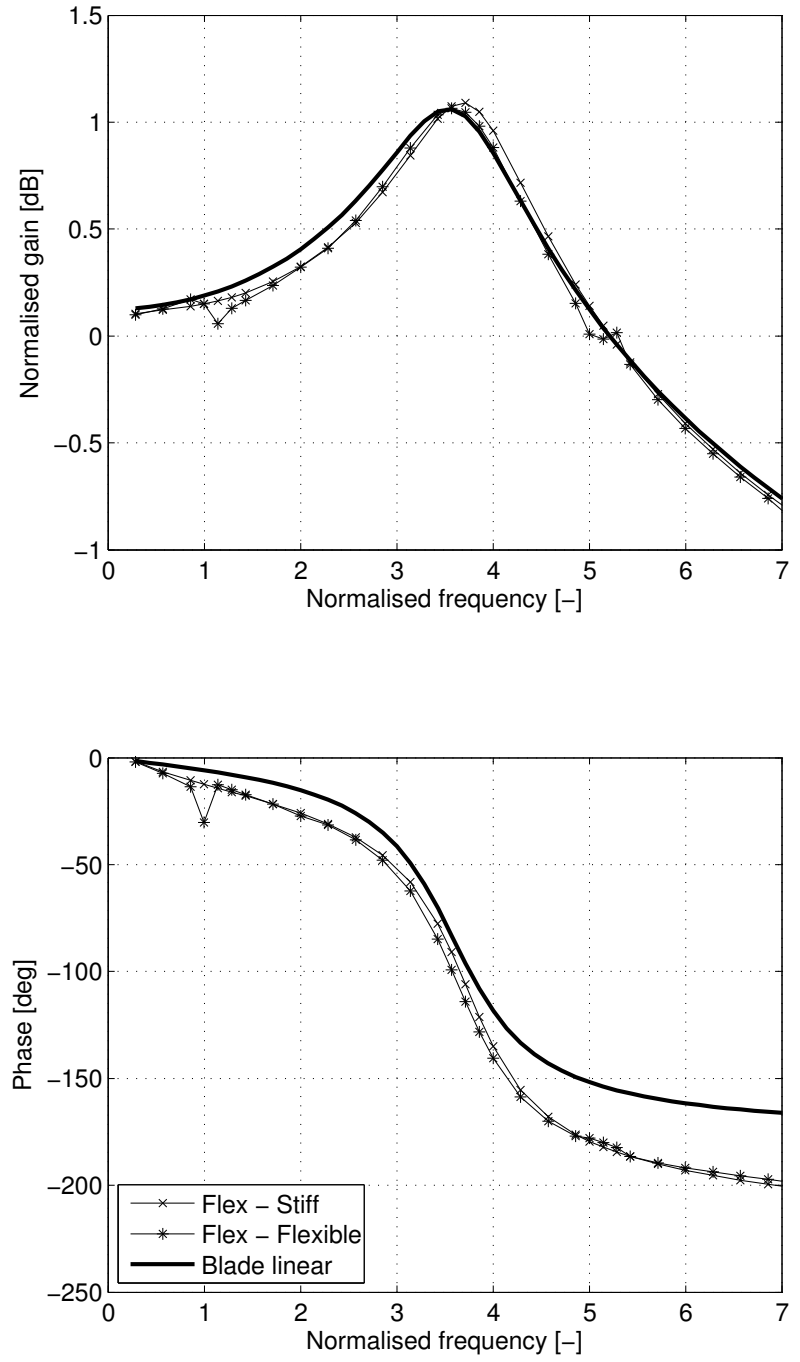

Fig. 3. Bode plot for the SISO system trailing edge flap angle to flapwise blade root moment. The $\mathrm{x}$ markers refer to simulations run in Flex, with a stiff turbine where only the first blade eigenmode is flexible. The star markers refer to simulations run in Flex with the model of a turbine where all the main turbine components are modeled as flexible structures. The thick line refers to the analytical linear model as described in this paper. The frequencies are normalised with the $1 \mathrm{P}$ frequency.

blades out of the wind when power production exceeds the turbine nominal power. The trailing edge flaps controller runs in parallel to the pitch controller, and aims at reducing the flapwise blade root loads only (Fig. 6). A low-level PID controller designed by the actuator manufacturer ensures that the actuators follow the target signal from the trailing edge flap controller at frequencies up to around 3P.

The trailing edge flaps controller described in this paper is a linear model predictive control (MPC). Model predictive control is the only advanced control technique that has been widely used in industrial process control [29]. Non linear MPC would be too slow to run real-time at a sampling frequency high enough to limit the time delays in the system. Linear MPC is chosen instead, and gain scheduling is used to deal with some of the non linearities of the system.

In the linear MPC described in this paper, an optimal time series of inputs $\hat{U}=[\hat{u}(k+1 \mid k), \ldots, \hat{u}(k+N \mid n)]^{\prime}$ minimising 

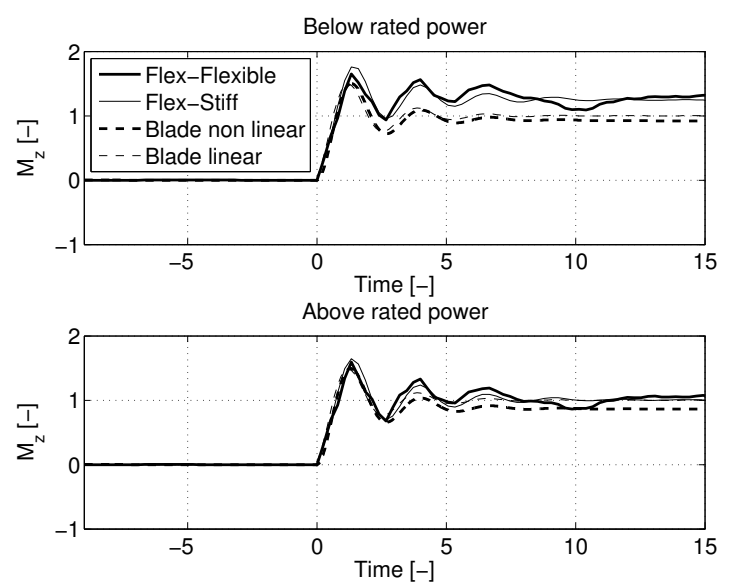

Fig. 4. Discrete change in wind speed. Time series of blade root flap moment $M_{z}$, when a discrete change in wind speed is introduced at time $t=0$. Four models are used: the Flex V27 model where all the turbine components are modeled as flexible (thick black line), the Flex V27 model where only the blade first mode is kept flexible (black line), the blade non linear model (thick dash line) and the blade linear model (dash line)
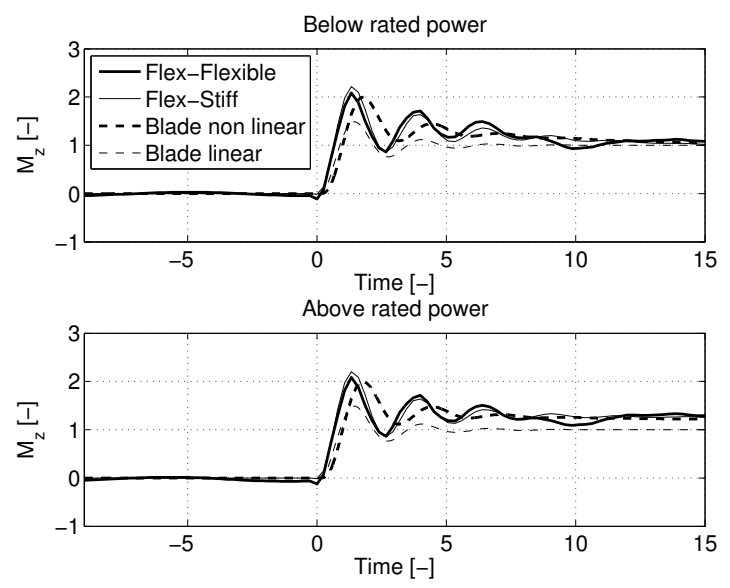

Fig. 5. Discrete change in trailing edge flap angle. Time series of blade root flap moment $M_{z}$, when a discrete change in trailing edge flap angle is introduced at time $t=0$. Four models are used: the Flex V27 model where all the turbine components are modeled as flexible (thick black line), the Flex V27 model where only the blade first mode is kept flexible (thin black line), the blade non linear model (thick dash line) and the blade linear model (thin dash line).

a quadratic cost function $\Psi(k)$ is calculated, at each time step:

$$
\begin{array}{ll}
\min _{\hat{U}} & \Psi(k)=\frac{1}{2} \hat{U}^{\prime} H \hat{U}+g^{\prime} \hat{U} \\
\text { s.t. } & U_{\min } \leq \hat{U} \leq U_{\max }
\end{array}
$$

where $\hat{u}(k+i \mid k)$ is the predicted optimal input at time $k+i$, calculated at the time step $k, N$ is the horizon length, $U_{\min }$ and $U_{\max }$ are the constraints on the inputs. The matrices $H$ and $g$ describe the quadratic cost function, they are function of, amongst others, the predicted disturbances and the system states. Only the first computed input $\hat{u}(k+1 \mid k)$ is used as a target for the actuators, and the optimisation problem is solved again at the next time step, updated with new measurements.

The convex optimisation problem is solved using qpOASES,

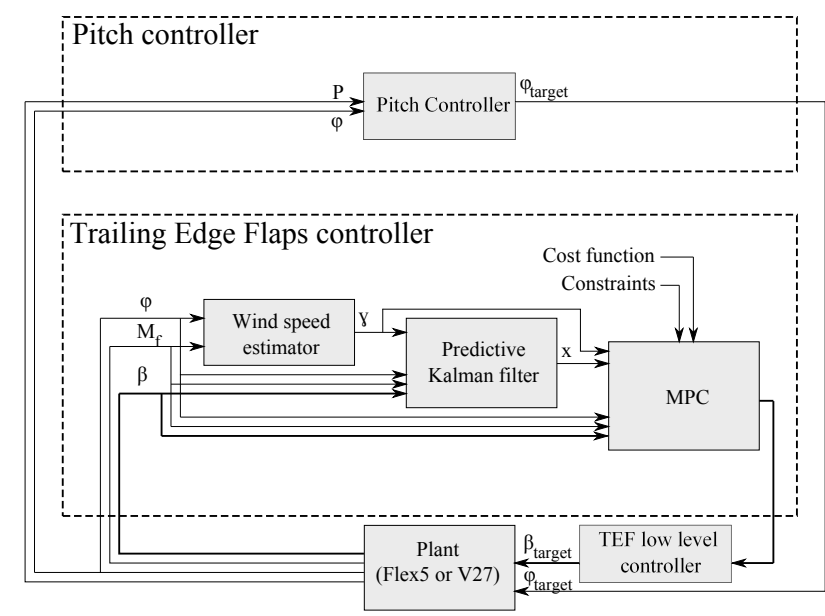

Fig. 6. Illustration of the pitch controller and the trailing edge flaps controller of the V27 wind turbine.

an open-source quadratic program suited for model predictive control [30], [31].

Linear MPC is a well-known control theory. This paper focuses on the aspects of the MPC which are specific to this application: the disturbance model which is required in order to improve the prediction of the outputs (Section V-C), the predictive Kalman filter which estimates the design model states. including the disturbance states (Section V-D), the gain scheduling (Section V-B) and the different quadratic cost functions which allows frequency-weighted control with MPC (Sections VI-A, VI-B and VI-C).

\section{B. Wind speed estimator and gain scheduling}

The linear blade model (8a) to (8d) is a function of an estimated mean free wind speed $\gamma$. No wind speed sensors are available for the trailing edge flaps controller in the test turbine. The mean free wind speed $V$ seen by the V27 turbine is estimated from a look-up table function of the pitch angle and the flap blade root moment of the 3 blades: below rated power, the blade root flapwise moment increases as a function of the mean wind speed, and the pitch angle remains close to 0 degree, whereas above rated power, the blades are pitched out of the wind and the blade root flapwise moment decreases as the wind speed increase. A low pass filter ensures that the estimated effective wind speed $\gamma$ is a smooth function of time. The linear blade model (8a) to (8d) is a function of an estimated mean free wind speed $\gamma$. No wind speed sensors are available for the trailing edge flaps controller in the test turbine. The mean free wind speed $V$ seen by the V27 turbine is estimated from a look-up table function of the pitch angle and the flap blade root moment of the 3 blades: below rated power, the blade root flapwise moment increases as a function of the mean wind speed, and the pitch angle remains close to 0 degree, whereas above rated power, the blades are pitched out of the wind and the blade root flapwise moment decreases as the wind speed increase. A low pass filter ensures that the estimated effective wind speed $\gamma$ is a smooth function of time. 


\section{Disturbance states}

The linear blade model (8a) to (8d) does not include any model of the wind speed seen by the rotating blade, which is at the origin of most of the flapwise blade root fatigue loads targeted by the model predictive control. It is added to the blade model as a disturbance model, where the wind speed seen by the spinning blade is modeled by its first harmonics, using sine functions with the $1 \mathrm{P}, 2 \mathrm{P}$ and $3 \mathrm{P}$ frequencies. An extra input disturbance state, an integral of a white noise, is added to handle a quasi steady-state plant-model mismatch. The Kalman filter is used to estimate those disturbance states, including the amplitude and the phase of the 3 harmonics of the wind speed seen by the rotating blade.

The augmented system is

$$
\begin{aligned}
{\left[\begin{array}{c}
\dot{\mathbf{x}}_{b} \\
\dot{\zeta}
\end{array}\right]=} & {\left[\begin{array}{cc}
\mathbb{A}_{b}(\gamma) & \mathbb{G}_{\zeta}(\gamma) \\
0 & \mathbb{A}_{\zeta}(\gamma)
\end{array}\right]\left[\begin{array}{c}
\mathbf{x}_{b} \\
\zeta
\end{array}\right]+\left[\begin{array}{c}
\mathbb{B}_{b}(\gamma) \\
0
\end{array}\right] \mathbf{u} } \\
& +\left[\begin{array}{c}
\mathbb{G}_{b}(\gamma) \\
0
\end{array}\right] \mathbf{d} \\
\mathbf{z}= & {\left[\begin{array}{ll}
\mathbb{C}_{b} & 0
\end{array}\right]\left[\begin{array}{c}
\mathbf{x}_{b} \\
\zeta
\end{array}\right] } \\
\mathbf{y}= & {\left[\begin{array}{ll}
\mathbb{C}_{b}^{m} & 0
\end{array}\right]\left[\begin{array}{c}
\mathbf{x}_{b} \\
\zeta
\end{array}\right] }
\end{aligned}
$$

where $\mathbb{A}_{\zeta}(\gamma)$ and $\mathbb{G}_{\zeta}(\gamma)$ are the disturbance model matrices.

The system (12a) to (12c) is discretised at the controller sampling frequency $(50 \mathrm{~Hz})$ :

$$
\begin{aligned}
x_{k+1} & =A(\gamma) x_{k}+B(\gamma) u_{k}+G(\gamma) d_{k} \\
z_{k} & =C x_{k} \\
y_{k} & =C^{m} x_{k}
\end{aligned}
$$

where $x_{k}$ is the state vector, $u_{k}$ is the control input, $d_{k}$ is the disturbance vector, $z_{k}$ is the output vector and $y_{k}$ is the measured output vector.

\section{Kalman filter}

None of the states $x_{k}$ are measured. A Kalman filter is used to estimate both the blade states and the disturbance states. The predictive Kalman filter is prefered over the ordinary filter in order to ease the implementation of the controller in the real turbine.

White noises are added to the design model:

$$
\begin{aligned}
x_{k+1} & =A(\gamma) x_{k}+B(\gamma) u_{k}+G(\gamma) d_{k}+w_{k} \\
y_{k} & =C^{m} x_{k}+v_{k}
\end{aligned}
$$

where $w_{k}$ is the process white noise, $w_{k}\left(0, Q_{k}(\gamma)\right)$, and $v_{k}$ is the observation noise, $v_{k}\left(0, R_{k}(\gamma)\right)$.

A first tuning of the covariance matrices $Q_{k}(\gamma)$ and $R_{k}(\gamma)$ is made by running simulations [32]. The blade states can be compared to the values provided by Flex5. The noise variance of the disturbance states depends on the wind conditions. The noise variances associated to the periodic states have to be low enough to make sure that those states have the desired $1 \mathrm{P}, 2 \mathrm{P}$ and $3 \mathrm{P}$ frequencies, and high enough to allow the amplitude and the phase of those quasi-sine functions to change fast enough to capture well the change in wind shear or yaw direction for example.
At each time step $k, \hat{x}_{k+1 \mid k}$ is the states estimates at the next time step $k+1$, based on the inputs $u_{k}$, the disturbances $d_{k}$ and the measurements $z_{k}$ at time step $k$.

$$
\begin{aligned}
\hat{x}_{k+1 \mid k}=( & \left.(\gamma)-K(\gamma) C^{m}\right) \hat{x}_{k \mid k-1} \\
& +B(\gamma) u_{k}+G(\gamma) d_{k}+K(\gamma) y_{k}
\end{aligned}
$$

where

$$
K(\gamma)=A(\gamma) P(\gamma) C^{m^{\prime}}\left(C^{m} P(\gamma) C^{m^{\prime}}+R_{k}(\gamma)\right)^{-1}
$$

and $P(\gamma)$ is the solution of the discrete time algebraic Riccati equation $A(\gamma) P(\gamma) A^{\prime}(\gamma)-P(\gamma)-K(\gamma) C^{m} P(\gamma) A^{\prime}(\gamma)+$ $Q_{k}(\gamma)=0$.

\section{E. Implementation}

The model predictive controller is coded in $\mathrm{C}++$, using the CBLAS [33] library for performing efficient algebra calculations, and the quadratic program qpOASES developped at K. U. Leuven [30]. The same $\mathrm{C}++$ code can be compiled into either a DLL which can be run with the wind turbine simulation code Flex5, or into a shared library for use with the cRio 9024. The shared library is linked to a Labview code which runs on the cRio 9024.

\section{COST FUnCTIONS FOR FREQUENCY WEIGTHED MPC}

\section{A. Costs on filtered inputs and outputs}

1) Motivation: Conventional model predictive control penalises variation of the predicted outputs from the reference trajectory, regardless of the frequencies of the variations. The control problem stated in this paper consists in alleviating only the loads with frequencies between the $1 \mathrm{P}$ and the $3 \mathrm{P}$ frequencies. The cost function derived in this section includes costs on filtered inputs and outputs, in order to emphasise some frequency regions. This method requires to derive the predicted filtered inputs and outputs as a function of the predicted inputs, the predicted disturbances, the model states and the filter states.

2) Predicted outputs: The predicted outputs are calculated recursively from (13a) and (13b):

$$
\hat{Z}=\Phi(\gamma) x_{1}+\Gamma(\gamma) \hat{U}+\Gamma_{d}(\gamma) \hat{D}
$$

where $\hat{Z}$ is the vector of predicted outputs $\hat{Z}:=$ $\left[\begin{array}{lll}\hat{z}(k+1 \mid k) & \ldots & \hat{z}(k+N \mid k)\end{array}\right]^{\prime}, \hat{U}$ is the vector of predicted inputs $\hat{U}:=\left[\begin{array}{lll}\hat{u}(k+1 \mid k) & \ldots & \hat{u}(k+N \mid k)\end{array}\right]^{\prime}$ and $\hat{D}$ is the vector of predicted disturbances $\hat{D}:=$ $\left[\begin{array}{lll}\hat{d}(k+1 \mid k) & \ldots & \hat{d}(k+N \mid k)\end{array}\right]^{\prime} . N$ is the horizon length of the model predictive control. $x_{1}$ is the vector of the design model states. The estimated mean free wind speed $\gamma$ 
is assumed constant over the horizon length.

$$
\begin{aligned}
& \Phi(\gamma)=\left[\begin{array}{c}
C A \\
C A^{2} \\
\vdots \\
C A^{N}
\end{array}\right] \\
& \Gamma(\gamma)=\left[\begin{array}{cccc}
C B & 0 & \ldots & 0 \\
C A B & C B & \ddots & \vdots \\
\vdots & \ddots & \ddots & 0 \\
C A^{N-1} B & \ldots & C A B & C B
\end{array}\right] \text {. }
\end{aligned}
$$

$\Gamma_{d}$ is similar to $\Gamma$, with the $G$ matrix replacing $B$. For clarifying the notations, the dependence on $\gamma$ is sometimes omitted in the right hand side of the equations.

3) Predicted filtered inputs: Let's define a state-space, discrete time, filter on the predicted inputs $(\tilde{A}, \tilde{B}, \tilde{C}, \tilde{D})$ :

$$
\begin{aligned}
x_{u}(k+i+1 \mid k) & =\tilde{A} x_{u}(k+i \mid k)+\tilde{B} \hat{u}(k+i \mid k) \\
\tilde{u}(k+i \mid k) & =\tilde{C} x_{u}(k+i \mid k)+\tilde{D} \hat{u}(k+i \mid k) .
\end{aligned}
$$

$\tilde{u}(k+i \mid k)$ is the predicted filtered input at time $k+i$, assumed at time $k$.

The vector of predicted filtered inputs $\tilde{U}:=$ $\left[\begin{array}{lll}\tilde{u}(k+1 \mid k) & \ldots & \tilde{u}(k+N \mid k)\end{array}\right]^{\prime}$ is

$$
\tilde{U}=\Phi_{\tilde{u}} x_{u}(k+1 \mid k)+\Gamma_{\tilde{u}} \hat{U}
$$

with

$$
\Phi_{\tilde{u}}=\left[\begin{array}{c}
\tilde{C} \\
\tilde{C} \tilde{A} \\
\vdots \\
\tilde{C} \tilde{A}^{N-1}
\end{array}\right], \Gamma_{\tilde{u}}=\left[\begin{array}{cccc}
\tilde{D} & 0 & \ldots & 0 \\
\tilde{C} \tilde{B} & \tilde{D} & 0 & 0 \\
\vdots & \ddots & \ddots & 0 \\
\tilde{C} \tilde{B}^{N-1} & \ldots & \tilde{C} \tilde{B} & \tilde{D}
\end{array}\right] .
$$

4) Predicted filtered outputs: The same way, the filtered predicted outputs $\tilde{Z}:=\left[\begin{array}{lll}\tilde{z}(k+1 \mid k) & \ldots & \tilde{z}(k+N \mid k)\end{array}\right]^{\prime}$ are calculated as a function of the predicted outputs:

$$
\tilde{Z}=\Phi_{\tilde{z}} x_{z}(k+1 \mid k)+\Gamma_{\tilde{z}} \hat{Z} .
$$

Combining (15) and (18) yields

$$
\tilde{Z}=\Phi_{\tilde{z}} x_{z}(k+1 \mid k)+\Gamma_{\tilde{z}}\left(\Phi(\gamma) x_{1}+\Gamma(\gamma) \hat{U}+\Gamma_{d}(\gamma) \hat{D}\right)
$$

5) Cost function: The cost function $\Psi(k)$ is the sum of a quadratic cost on the difference between the filtered predicted outputs and the set points $\Psi_{\tilde{z}}(k)$ and a quadratic cost on the filtered predicted inputs $\Psi_{\tilde{u}}(k)$ :

$$
\begin{gathered}
\Psi(k)=\sum_{i=1}^{N}\|\tilde{z}(k+i \mid k)-r(k+i \mid k)\|_{Q_{\tilde{z}}}^{2} \\
+\sum_{i=1}^{N}\|\tilde{u}(k+i \mid k)\|_{Q_{\tilde{u}}}^{2}
\end{gathered}
$$

or, in the matrix form

$$
\Psi(k)=\frac{1}{2}(\tilde{Z}-R)^{\prime} \mathcal{Q}_{\tilde{z}}(\tilde{Z}-R)+\frac{1}{2} \tilde{U}^{\prime} \mathcal{Q}_{\tilde{u}} \tilde{U} .
$$

$\mathcal{Q}_{\tilde{z}}=I_{N} \otimes Q_{\tilde{z}}$ and $\mathcal{Q}_{\tilde{u}}=I_{N} \otimes Q_{\tilde{u}}$, assuming constant costs on the filtered inputs $Q_{\tilde{u}}$ and on the filetred output $Q_{\tilde{z}}$ along the horizon length. $\otimes$ denotes the Kronecker product.

Combining (17), (19) and (21):

$$
\Psi(k) \equiv \frac{1}{2} \hat{U}^{\prime}\left(H_{\tilde{z}}(\gamma)+H_{\tilde{u}}\right) \hat{U}+g(\gamma)^{\prime} \hat{U}
$$

with

$$
\begin{aligned}
g(\gamma):= & M_{\tilde{z}, R}(\gamma) R+M_{\tilde{z}, D}(\gamma) \hat{D}+M_{\tilde{z}, x_{1}}(\gamma) x_{1} \\
& +M_{\tilde{z}, x_{\tilde{z}}}(\gamma) x_{z}(k+1 \mid k)+M_{\tilde{u}, x_{\tilde{u}}} x_{u}(k+1 \mid k)
\end{aligned}
$$

and

$$
\begin{aligned}
H_{\tilde{z}}(\gamma) & :=\Gamma^{\prime}(\gamma) \Gamma_{\tilde{z}}^{\prime} \mathcal{Q}_{\tilde{z}} \Gamma_{\tilde{z}} \Gamma(\gamma) \\
H_{\tilde{u}} & :=\Gamma_{\tilde{u}}^{\prime} \mathcal{Q}_{\tilde{u}} \Gamma_{\tilde{u}} \\
M_{\tilde{z}, R}(\gamma) & :=-\Gamma^{\prime}(\gamma) \Gamma_{\tilde{z}}^{\prime} \mathcal{Q}_{\tilde{z}} \\
M_{\tilde{z}, D}(\gamma) & :=\Gamma^{\prime}(\gamma) \Gamma_{\tilde{z}}^{\prime} \mathcal{Q}_{\tilde{z}} \Gamma_{\tilde{z}} \Gamma_{d}(\gamma) \\
M_{\tilde{z}, x_{1}}(\gamma) & :=\Gamma^{\prime}(\gamma) \Gamma_{\tilde{z}}^{\prime} \mathcal{Q}_{\tilde{z}} \Gamma_{\tilde{z}} \Phi(\gamma) \\
M_{\tilde{z}, x_{\tilde{z}}}(\gamma) & :=\Gamma^{\prime}(\gamma) \Gamma_{\tilde{z}}^{\prime} \mathcal{Q}_{\tilde{z}} \Phi_{\tilde{z}} \\
M_{\tilde{u}, x_{\tilde{u}}} & :=\Gamma_{\tilde{u}}^{\prime} \mathcal{Q}_{\tilde{u}}^{\prime} \Phi_{\tilde{u}} .
\end{aligned}
$$

The sign $\equiv$ denotes that the terms independent of $\hat{U}$ have been removed from the equation. Those terms are irrelevant when it comes to finding the minimum of the cost function $\Psi(k)$.

The matrices $H_{\tilde{z}}(\gamma), H_{\tilde{u}}, M_{\tilde{z}, R}(\gamma), M_{\tilde{z}, D}(\gamma), M_{\tilde{z}, x_{1}}(\gamma)$, $M_{\tilde{z}, x_{\tilde{z}}}(\gamma)$ and $M_{\tilde{u}, x_{\tilde{u}}}$ are all calculated offline, for several mean free wind speeds $\gamma$. Finding the minimum of the cost function (21) is a quadratic program of dimension $N$, similar to the one for conventional linear model predictive control. The only additional operations to carry out at each time step are the matrix multiplications $M_{\tilde{z}, x_{\tilde{z}}}(\gamma) x_{z}(k+1 \mid k)$ and $M_{\tilde{u}, x_{\tilde{u}}} x_{u}(k+1 \mid k)$.

6) Results: In order to evaluate the different cost functions presented in this paper, a 10 minute simulation of a below rated normal production load case is run with the different frequency-weighted model predictive control cost functions presented in this paper. Comparing the spectral densities of the blade root flap moment and the trailing edge flap angle gives an estimate of the performance of the controller. A more rigorous way to evaluate the performance of the controller is nevertheless to calculate the blade root flapwise fatigue loads by the use of the rainflow counting method and the Wöhler curve of the material. The trailing edge flap travel distance and the mean power production are calculated as well.

Fig. 7 shows the spectral densities of the blade root flap moment and the trailing edge flap angle, for the baseline case and for the cases where the flaps are actuated with the MPC previously described, with the use of low-order (second order) and high-order (eighth order) filters. Both controllers reduce significantly the amplitude of the $1 \mathrm{P}, 2 \mathrm{P}$ and $3 \mathrm{P}$ loads. But the use of high-order filters in the cost function shows an increase of the loads at frequencies between 4 and 4.5P. This is the consequence of the introduction by the output filters in the cost function of too large time lags in the system. The time lag of the output filter at such frequencies is around -130 degrees. As a consequence, the blade root fatigue loads are only reduced 

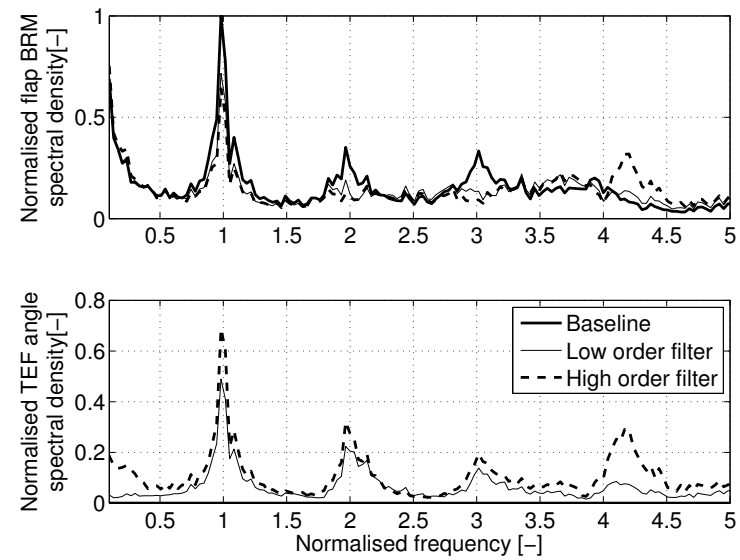

Fig. 7. Flapwise blade root moment spectral density (top) and trailing edge flap angle spectral density (bottom) of a 10 minute simulation. MPC with costs on filtered inputs and outputs is used to control the trailing edge flaps. The use of high order filters in the cost function results in an increase of the flapwsie blade root loads at frequencies between 4 and $4.5 \mathrm{P}$.

by $0.8 \%$ when high-order filters are used, whereas they are reduced by $6.2 \%$ with the low-order filters. The extra use of the trailing edge flaps at the 4P frequency increases the trailing edge flap travel distance by $77.7 \%$, compared to the simulation with the low order filters.

\section{B. Costs on zero-phase filtered inputs and outputs}

1) Motivation: Filters introduce time lags in the cost function, which reduces the performance of the controller with filters, and which forbids the use of high order filters. Such high-order filters could nevertheless be useful in order to be able to separate sharply the targeted frequencies, and avoid the unwished excitation of some frequencies. Forward-backward filters, which consists in filtering the data forward, and then filtering the filtered data backwards, are zero-phase filters. Implementation of such a method in a MPC cost function is possible because of the finite horizon length used in MPC. Special attention is put on the filter initial conditions in order to minimise transients.

2) Zero-phase filtered predicted inputs: At each time step $k$, the zero-phase filtering is run on a set of data which includes the measured inputs in the past $U_{m}$ ( $N_{U, m}$ samples), and the predicted inputs within the horizon length $\hat{U}$ ( $N$ samples). Gustafsson's method to minimise startup and ending transient implies reflecting the first $N_{u, r}$ samples of $U_{m}$ and the last $N_{u, r}$ samples of $\hat{U}$ [34]. The zero-phase filtering is then applied to the inputs vector $U_{a}:=\left[\begin{array}{llll}U_{m, r} & U_{m} & \hat{U} & \hat{U}_{r}\end{array}\right]^{\prime}$, with $U_{m, r}$ and $\hat{U}_{r}$ being respectively the first $N_{u, r}$ reflected samples of $U_{m}$ and the last $N_{u, r}$ reflected samples of $\hat{U}$. The size of the vector $U_{a}$ is $N_{U a}=n_{u}\left(N_{U, m}+N+2 N_{u, r}\right)$.

In this section, the system $(\tilde{A}, \tilde{B}, \tilde{C}, \tilde{D})$ denotes the inputs filter. The right arrow $\rightarrow$ is applied to variables refering to the forward filtering, the left arrow $\leftarrow$ to variables refering to backward filtering and the left-right arrow $\leftrightarrow$ to variables refering to forward-backward filtering.

The vector $U_{a}$ is first filtered forward. The filtered vector $\overrightarrow{U_{a}}$ is a function of the non filtered vector $U_{a}$ and the initial states of the filter $\overrightarrow{x_{u, 1}}$.

$$
\overrightarrow{U_{a}}:=\overrightarrow{\Gamma_{u}} U_{a}+\overrightarrow{\Phi_{u}} \overrightarrow{x_{u, 1}}
$$

where

$$
\begin{aligned}
\vec{\Gamma}_{u}:= & {\left[\begin{array}{ccccc}
\tilde{D} & 0 & 0 & \ldots & 0 \\
\tilde{C} \tilde{B} & \tilde{D} & 0 & \ldots & 0 \\
\tilde{C} \tilde{A} \tilde{B} & \tilde{C} \tilde{B} & \tilde{D} & 0 & 0 \\
\vdots & \ddots & \ddots & \ddots & 0 \\
\tilde{C} \tilde{A}^{N_{U a}-2} \tilde{B} & \ldots & \tilde{C} \tilde{A} \tilde{B} & \tilde{C} \tilde{B} & \tilde{D}
\end{array}\right] } \\
\vec{\Phi}_{u}:= & {\left[\begin{array}{c}
\tilde{C} \\
\tilde{C} \tilde{A} \\
\vdots \\
\tilde{C} \tilde{A}^{N_{U a}-1}
\end{array}\right] . }
\end{aligned}
$$

(24) is split into two parts: one related to the measured inputs $U_{m, r}$ and $U_{m}$, and one related to the predicted inputs $\hat{U}$ and $\hat{U}_{r}$ :

$$
\left[\begin{array}{c}
\overrightarrow{U_{m_{r} r}} \\
\overrightarrow{U_{m}} \\
\hline \overrightarrow{\hat{U}} \\
\overrightarrow{\hat{U}_{r}}
\end{array}\right]=\left[\begin{array}{c|c}
\overrightarrow{\Gamma_{u, 11}} & {[0]} \\
\hline \overrightarrow{\Gamma_{u, 21}} & \overrightarrow{\Gamma_{u, 22}}
\end{array}\right]\left[\begin{array}{c}
U_{m, r} \\
\frac{U_{m}}{\hat{U}} \\
\hat{U}_{r}
\end{array}\right]+\left[\begin{array}{c}
\overrightarrow{\phi_{u, 1}} \\
\overrightarrow{\phi_{u, 2}}
\end{array}\right] \overrightarrow{x_{u, 1}} .
$$

The vector $\overrightarrow{U_{a}}$ is then filtered backwards in order to get the zero-phase filtered inputs vector $\overleftrightarrow{U_{a}}$.

$$
\overleftrightarrow{U_{a}}:=\vec{\Gamma}_{u}^{\prime} \overrightarrow{U_{a}}+\overleftarrow{\Phi_{u}} \overleftarrow{x_{u, 1}}
$$

with

$$
\overleftarrow{\Phi_{u}}:=\left[\begin{array}{c}
\tilde{C} \tilde{A}^{N_{U a}-1} \\
\tilde{C} \tilde{A}^{N_{U a}-2} \\
\vdots \\
\tilde{C}
\end{array}\right]
$$

In order to minimise startup and ending transients, $\overrightarrow{x_{u, 1}}$ and $\overleftarrow{x_{u, 1}}$ are calculated as a function of $U_{a}(1)$, first sample of the $U_{a}$ vector, and $\overrightarrow{U_{a}}\left(N_{U a}\right)$, last sample of $\overrightarrow{U_{a}}$.

$$
\begin{aligned}
& \overrightarrow{x_{u, 1}}=z_{u} U_{a}(1) \\
& \overleftarrow{x_{u, 1}}=z_{u} \overrightarrow{U_{a}}\left(N_{U a}\right) .
\end{aligned}
$$

$z_{u}$ is a vector which is a function of the inputs filter only [34], and is calculated offline. The last sample of $\overrightarrow{U_{a}}$ is calculated as:

$$
\overrightarrow{U_{a}}\left(N_{U a}\right)=\left[\overrightarrow{\Gamma_{u, 21, e}} \mid \overrightarrow{\Gamma_{u, 22, e}}\right]\left[\begin{array}{c}
U_{m, r} \\
\frac{U_{m}}{\hat{U}} \\
\hat{U}_{r}
\end{array}\right]+\overrightarrow{\phi_{u, 2, e}} \overrightarrow{x_{u, 1}}
$$

where $\overrightarrow{\Gamma_{u, 21, e}}, \overrightarrow{\Gamma_{u, 22, e}}$ and $\overrightarrow{\phi_{u, 2, e}}$ are respectively the $n_{u}$ last lines of $\overrightarrow{\Gamma_{u, 21}}, \overrightarrow{\Gamma_{u, 22}}$ and $\overrightarrow{\phi_{u, 2}}$. 
The matrices $I_{\overleftrightarrow{u}, 0}, I_{\overleftrightarrow{u}, 1}, I_{\overleftrightarrow{u}, r}$, and $I_{\overleftrightarrow{u}, m, r}$ are defined as

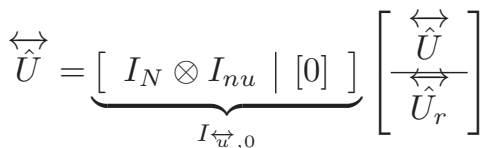

$$
\begin{aligned}
& U_{a}(1)=\underbrace{\left[\begin{array}{ll}
I_{n u} & {[0]}
\end{array}\right]}_{I_{\overleftrightarrow{u}, 1}}\left[\begin{array}{c}
U_{m, r} \\
U_{m}
\end{array}\right] \\
& {\left[\begin{array}{c}
\hat{U} \\
\hat{U}_{r}
\end{array}\right]=\underbrace{\left[\begin{array}{cccc}
I_{n u} & & & \\
& I_{n u} & & \\
& & \ddots & \\
& & & I_{n u} \\
& -I_{n u} & -I_{n u} & 2 I_{n u} \\
. \cdot & & 2 I_{n u} \\
& \vdots &
\end{array}\right]}_{I_{\overleftrightarrow{u}, r}} \hat{U}}
\end{aligned}
$$

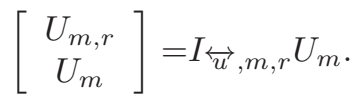

Finally, the predicted zero-phase filtered inputs are function only of the measured inputs $U_{m}$ and the predicted inputs $\hat{U}$ :

$$
\overleftrightarrow{\hat{U}}=\Delta_{\overleftrightarrow{U}}^{\overleftrightarrow{U}} \hat{U}+\Delta_{U_{m}}^{\overleftrightarrow{U}} U_{m}
$$

with

$$
\begin{aligned}
& \Delta_{\overleftrightarrow{U}}^{\overleftrightarrow{U}}:=I \overleftrightarrow{u}, 0 {\left[\overrightarrow{\Gamma_{u, 22}}, \overrightarrow{\Gamma_{u, 22}}+\overleftrightarrow{\phi_{u, 2}} z_{u} \overrightarrow{\Gamma_{u, 22, e}}\right] I_{\overleftrightarrow{u}, r} } \\
& \Delta_{U_{m}}^{\overleftrightarrow{U}}:=I_{\overleftrightarrow{u}, 0}\left[\overrightarrow{\Gamma_{u, 22}},\left(\overrightarrow{\Gamma_{u, 21}}+\overrightarrow{\phi_{u, 2}} z_{u} I_{\overleftrightarrow{u}, 1}\right)\right. \\
&\left.+\overleftarrow{\phi_{u, 2}} z_{u}\left(\overrightarrow{\Gamma_{u, 21, e}}+\overrightarrow{\phi_{u, 2, e}} z_{u} I_{\overleftrightarrow{u}, 1}\right)\right] I_{\overleftrightarrow{u}, m, r} .
\end{aligned}
$$

3) Zero-phase filtered predicted outputs: The zero-phase filtered predicted outputs $\overleftrightarrow{Z}$ are derived with the same method as for the zero-phase filtered inputs.

$$
\overleftrightarrow{Z}:=\Delta_{\hat{Z}}^{\overleftrightarrow{Z}} \hat{Z}+\Delta_{Z_{m}}^{\overleftrightarrow{Z}} Z_{m}
$$

with

$$
\begin{aligned}
\Delta_{\hat{Z}}^{\overleftrightarrow{Z}}:=I_{\overleftrightarrow{z}, 0} & {\left[\overrightarrow{\Gamma_{z, 22}}, \overrightarrow{\Gamma_{z, 22}}+\overleftarrow{\phi_{z, 2}} z_{z} \overrightarrow{\Gamma_{z, 22, e}}\right] I_{\overleftrightarrow{z}, r} } \\
\Delta_{Z_{m}}:=I_{\overleftrightarrow{z}, 0} & {\left[\overrightarrow{\Gamma_{z, 22}},\left(\overrightarrow{\Gamma_{z, 21}}+\overrightarrow{\phi_{z, 2}} z_{z} I_{\overleftrightarrow{z}, 1}\right)\right.} \\
+ & \left.\overleftarrow{\phi_{z, 2}} z_{z}\left(\overrightarrow{\Gamma_{z, 21, e}}+\overrightarrow{\phi_{z, 2, e}} z_{z} I_{\overleftrightarrow{z}, 1}\right)\right] I_{\overleftrightarrow{z}, m, r}
\end{aligned}
$$

Combining (15) and (40) yields

$$
\overleftrightarrow{Z}=\Delta_{Z_{m}}^{\overleftrightarrow{Z}} Z_{m}+\Delta_{\tilde{Z}}^{\overleftrightarrow{Z}} \Phi(\gamma) x_{1}+\Delta_{\tilde{Z}}^{\overleftrightarrow{Z}} \Gamma_{d}(\gamma) \hat{D}+\Delta_{\tilde{Z}}^{\overleftrightarrow{Z}} \Gamma(\gamma) \hat{U}
$$

4) Cost function: The new cost function $\Psi(k)$ including costs only on zero-phase filtered inputs and outputs is

$$
\begin{gathered}
\Psi(k):=\sum_{i=1}^{N}\|\overleftrightarrow{z}(k+i \mid k)-r(k+i \mid k)\|_{Q_{\overleftrightarrow{z}}}^{2} \\
+\sum_{i=1}^{N}\|\overleftrightarrow{u}(k+i \mid k)\|_{Q_{\overleftrightarrow{u}}}^{2}
\end{gathered}
$$

or, in the matrix form,

$$
\Psi(k)=\frac{1}{2}(\overleftrightarrow{Z}-R)^{\prime} \mathcal{Q} \overleftrightarrow{z}(\overleftrightarrow{Z}-R)+\frac{1}{2} \overleftrightarrow{U}^{\prime} \mathcal{Q}_{\overleftrightarrow{u}} \overleftrightarrow{U}
$$

$\mathcal{Q}_{\overleftrightarrow{z}}:=I_{N} \otimes Q_{\overleftrightarrow{z}}$ and $\mathcal{Q}_{\overleftrightarrow{u}}:=I_{N} \otimes Q_{\overleftrightarrow{u}}$, assuming constant costs on the filtered inputs $Q_{\overleftrightarrow{u}}$ and on the filetred output $Q \overleftrightarrow{z}$ along the horizon length.

Combining (37), (43) and (45) leads to the cost function

$$
\Psi(k) \equiv \frac{1}{2} \hat{U}^{\prime}(H \overleftrightarrow{Z}(\gamma)+H \overleftrightarrow{U}) \hat{U}+g(\gamma)^{\prime} \hat{U}
$$

with

$$
\begin{aligned}
g(\gamma):= & M_{\overleftrightarrow{z}, Z_{m}}(\gamma) Z_{m}+M_{\overleftrightarrow{z}, x_{1}}(\gamma) x_{1}+M_{\overleftrightarrow{z}, D}(\gamma) \hat{D} \\
& +M_{\overleftrightarrow{z}, R}(\gamma) R+M_{\overleftrightarrow{u}, U_{m}} U_{m}
\end{aligned}
$$

and

$$
\begin{aligned}
& H_{\overleftrightarrow{Z}}(\gamma):=\Gamma^{\prime}(\gamma) \Delta_{\hat{Z}} \overleftrightarrow{Z}^{\prime} \mathcal{Q}_{\overleftrightarrow{z}} \Delta_{\hat{Z}}^{\overleftrightarrow{Z}} \Gamma(\gamma) \\
& H_{\overleftrightarrow{U}}:=\Delta_{\hat{U}^{\prime}}^{\overleftrightarrow{U^{\prime}}} \mathcal{Q}_{\overleftrightarrow{u}} \Delta_{\overleftrightarrow{U}}^{\overleftrightarrow{U}} \\
& M_{\overleftrightarrow{z}, Z_{m}}(\gamma):=\Gamma^{\prime}(\gamma) \Delta_{\hat{Z}}^{\overleftrightarrow{Z}^{\prime}} \mathcal{Q} \overleftrightarrow{z^{\prime}} \Delta_{Z_{m}}^{\overleftrightarrow{Z}} \\
& M_{\overleftrightarrow{z}, x_{1}}(\gamma):=\Gamma^{\prime}(\gamma) \Delta_{\tilde{Z}} \overleftrightarrow{Z}^{\prime} \mathcal{Q} \overleftrightarrow{z} \Delta_{\hat{Z}}^{\overleftrightarrow{Z}} \Phi(\gamma) \\
& M_{\overleftrightarrow{z}, D}(\gamma):=\Gamma^{\prime}(\gamma) \Delta_{\hat{Z}}^{\overleftrightarrow{Z}^{\prime}} \mathcal{Q}_{\overleftrightarrow{z}} \Delta_{\hat{Z}} \overleftrightarrow{Z} \Gamma_{d}(\gamma) \\
& M_{\overleftrightarrow{z}, R}(\gamma):=\Gamma^{\prime}(\gamma) \Delta_{\hat{Z}} \overleftrightarrow{Z}^{\prime} \mathcal{Q}_{\overleftrightarrow{z}}
\end{aligned}
$$

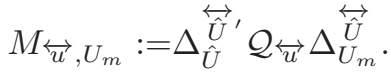

5) Results: Fig. 8 shows the performance of the MPC with high order filters as described in section VI-B and the MPC with zero-phase high order filters as described in this section. The same filters are used in both cases. The introduction of zero-phase filtering prevents the load increase at $4 \mathrm{P}$ which was seen with the MPC with conventional filters, without reducing the performance at frequencies $1 \mathrm{P}, 2 \mathrm{P}$ and $3 \mathrm{P}$. The use of zerophase filters also reduced the actuation of the flaps at very low frequencies (below 0.5P), which is important for fatigue load reduction: keeping the flaps at a 0 degree average gives them maximum amplitude to reduce the $1 \mathrm{P}$ load, at each rotor rotation.

As a consequence, the fatigue load reduction with zerophase filters is of $4.5 \%$, versus $0.8 \%$ with conventional filters. The trailing edge flaps travel distance remains very high $(76 \%$ higher than when low-order conventional filters are used). Increasing the costs on the zero-phase filtered outputs is thus possible, without leading to a load increase at high frequencies. This leads to an increase in the $1 \mathrm{P}$ load reduction, but also to an increase in the trailing edge flap travel distance at the $1 \mathrm{P}$ frequency. Trailing edge flaps activity at frequencies above $5 \mathrm{P}$ is also increased slightly.

C. Costs on the amplitude of the Fourrier transform of the inputs and outputs

1) Motivation: Costs on zero-phase filtered inputs and outputs allows the use of high-order filters, without being affected by the large time lags introduced bu those filters. Having costs on the amplitude of the Fourrier transform of 

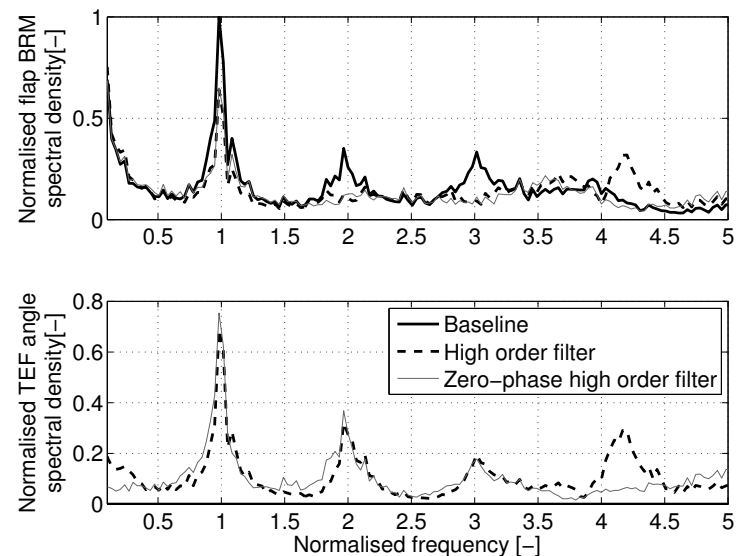

Fig. 8. Flapwise blade root moment spectral density (top) and trailing edge flap angle spectral density (bottom) of a 10 minute simulation. MPC with costs on high-order filtered inputs and outputs (thick dashed line) and on zero-phase high-order filtered inputs and outputs (thin grey line) are used to control the trailing edge flaps.

the inputs and the outputs is an even more flexible way to distribute weights on the different frequencies.

In order to simplify the equations and the notation, a single input single output system is considered in this section. But the method can be extrapolated to multiple inputs multiple outputs sytems.

2) Matrix form of the Fourier transform: The Discrete Fourier Transform (DFT) coefficients of a signal $x_{0}, \ldots, x_{N-1}$ are

$$
X_{k}:=\sum_{n=0}^{N-1} x_{n} e^{-i 2 \pi k \frac{n}{N}} \quad, k=0, \ldots, \mathrm{N}-1 .
$$

In a matrix form

$$
\left[\begin{array}{c}
X_{0} \\
\vdots \\
X_{N-1}
\end{array}\right]=\mathcal{F}\left[\begin{array}{c}
x_{0} \\
\vdots \\
x_{N-1}
\end{array}\right]
$$

with $\mathcal{F}_{k n}:=e^{\left(-i 2 \pi k \frac{n}{N}\right)}$ being the $(k, n)$ element of the $\mathcal{F}$ matrix.

The cost is distributed on the amplitude of the Fourrier transforms $\left\|X_{k}\right\|^{2}=\overline{X_{k}} X_{k}$, where $\overline{X_{k}}$ is the complex conjugate of $X_{k}$.

3) Cost on the amplitude of the DFT of the inputs: The DFT is applied to the vector $\mathcal{U}=\left[\begin{array}{c}U_{m} \\ \hat{U}\end{array}\right]$, where $U_{m}$ is the vector of measured inputs in the past, and $\hat{U}$ the vector of predicted inputs. The cost $\Psi_{\mathcal{U}_{\text {fft }}}(k)$ of the amplitudes of the DFT of the inputs is

$$
\begin{aligned}
\Psi_{\mathcal{U}_{\mathrm{fft}}}(k) & =\frac{1}{2} \overline{(\mathcal{F U})^{\prime}} \mathcal{Q}_{\mathcal{U}}(\mathcal{F U}) \\
& =\frac{1}{2} \mathcal{U}^{\prime} \overline{\mathcal{F}}^{\prime} \mathcal{Q}_{\mathcal{U}} \mathcal{F} \mathcal{U} .
\end{aligned}
$$

Notice that the cost is no longer in the time domain, but in the frequency domain. $\mathcal{Q}_{\mathcal{U}}$ is the matrix of weights on the different frequencies of the Fourier transform. $\mathcal{Q}_{\mathcal{U}}(f, f)$ is the cost on the amplitude of the input with a frequency of $(f-1) \frac{f_{s}}{N_{u}}$, where $f_{s}$ is the sampling frequency of the controller, and $N_{\mathcal{U}}$ is the size of the vector $\mathcal{U}$.

The matrix $\overline{\mathcal{F}}^{\prime} \mathcal{Q}_{\mathcal{U}} \mathcal{F}$ is complex symmetric with real diagonal. So

$$
\Psi_{\mathcal{U}_{\mathrm{fft}}}(k):=\frac{1}{2} \mathcal{U}^{\prime} \Re_{\mathcal{U}} \mathcal{U}
$$

where $\Re_{\mathcal{U}}$ is the real part of $\overline{\mathcal{F}}^{\prime} \mathcal{Q}_{\mathcal{U}} \mathcal{F}$.

$$
\begin{aligned}
\Psi_{\mathcal{U}_{\text {fft }}}(k) & =\frac{1}{2}\left[\begin{array}{c}
U_{m} \\
\hat{U}
\end{array}\right]^{\prime}\left[\begin{array}{ll}
\Re_{\mathcal{U}_{11}} & \Re_{\mathcal{U}_{12}} \\
\Re_{\mathcal{U}_{12}}^{\prime} & \Re_{\mathcal{U}_{22}}
\end{array}\right]\left[\begin{array}{c}
U_{m} \\
\hat{U}
\end{array}\right] \\
& \equiv \frac{1}{2} \hat{U}^{\prime} \Re_{\mathcal{U}_{22}} \hat{U}+\left[\Re_{\mathcal{U}_{12}}^{\prime} U_{m}\right]^{\prime} \hat{U} .
\end{aligned}
$$

4) Cost on the amplitude of the DFT of the outputs: With the same method and notations, the cost on the amplitude of the DFT of the outputs is

$$
\Psi_{\mathcal{Z}_{\mathrm{fft}}}(k) \equiv \frac{1}{2} \hat{Z}^{\prime} \Re_{\mathcal{Z}_{22}} \hat{Z}+\left[\Re_{\mathcal{Z}_{12}}^{\prime} Z_{m}\right]^{\prime} \hat{Z} .
$$

Combining (15) and (56) yields

$$
\begin{aligned}
\Psi_{\mathcal{Z}_{\mathrm{ftt}}}(k) & \equiv \frac{1}{2} \hat{Z}^{\prime} \Re_{\mathcal{Z}_{22}} \hat{Z}+\left[\Re_{\mathcal{Z}_{12}}^{\prime} Z_{m}\right]^{\prime} \hat{Z} \\
& \equiv \frac{1}{2} \hat{U}^{\prime} H_{\mathcal{Z}_{\mathrm{ftt}}} \hat{U}+g(\gamma)^{\prime} \hat{U}
\end{aligned}
$$

with

$$
g(\gamma):=M_{\mathcal{Z}_{\mathrm{ftt}}, x_{1}}(\gamma) x_{1}+M_{\mathcal{Z}_{\mathrm{ftt}}, \hat{D}}(\gamma) \hat{D}+M_{\mathcal{Z}_{\mathrm{ftt}}, Z_{m}}(\gamma) Z_{m}
$$

and

$$
\begin{aligned}
M_{\mathcal{Z}_{\text {fft }}, x_{1}}(\gamma) & :=\Gamma^{\prime}(\gamma) \Re_{\mathcal{Z}_{22}} \Phi(\gamma) \\
M_{\mathcal{Z}_{\mathrm{fft}}, \hat{D}}(\gamma) & :=\Gamma^{\prime}(\gamma) \Re_{\mathcal{Z}_{22}} \Gamma_{D}(\gamma) \\
M_{\mathcal{Z}_{\mathrm{fft}}, Z_{m}}(\gamma) & :=\Gamma^{\prime}(\gamma) \Re_{\mathcal{Z}_{12}}^{\prime} .
\end{aligned}
$$

5) Results: The use of costs on the amplitude of the DFT coefficients of the inputs and the outputs shows interesting results (Fig. 9). By penalising the trailing edge flap actuation below $0.5 \mathrm{P}$ and above $4 \mathrm{P}$, it is possible to reduce to almost 0 the use of the trailing edge flap at those frequencies, without altering the overall performance of the controller. As shown in Table I, this last controller is the best to reduce blade root fatigue loads $(-7.4 \%)$ with a minimum use of the actuators (24\% less than for the controller with costs on loworder filtered inputs and outputs). Please note that these load reductions are achieved with a $0.7 \mathrm{~m}$ long flap on the $13 \mathrm{~m}$ long V27 blade.

However, the good performance of the MPC with costs on the amplitude of the DFT coefficients of the inputs and the outputs comes at a cost: a large horizon length is required. The frequency step in the DFT of the signals is $\frac{f_{s}}{N_{\mathcal{U}}}$, which means that the size of $\mathcal{U}$ has to be large enough so that the frequency step is significantly smaller than the smallest frequency of interest (the $1 \mathrm{P}$ frequency). And, as the costs are in the frequency domain, and not in the time domain, it is important that the $\mathcal{U}$ vector includes several periods of the slowest periodic event (the $1 \mathrm{P}$ loads). A large $\mathcal{U}$ is thus required. The $\mathcal{U}$ vector is the concatenation of measured 

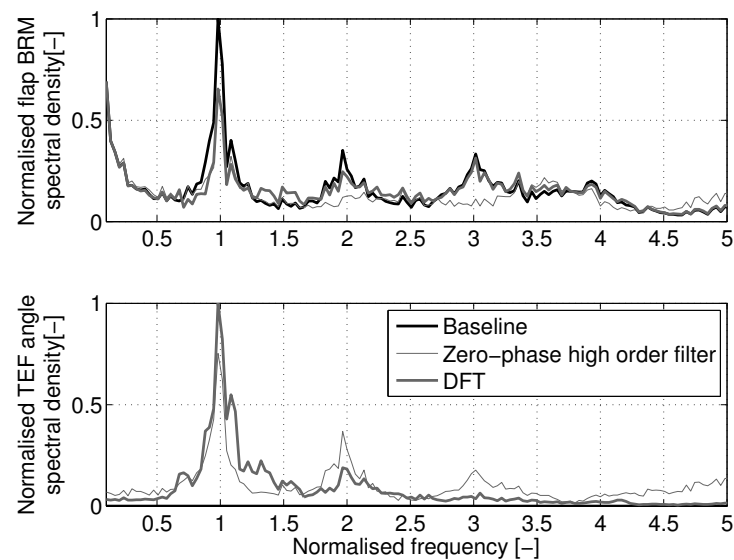

Fig. 9. Flapwise blade root moment spectral density (top) and trailing edge flap angle spectral density (bottom) of a 10 minute simulation. MPC with costs on zero-phase filtered inputs and outputs (thin grey line) and on the amplitude of the coefficients of the DFT of the inputs and outputs (thick grey line) are used to control the trailing edge flaps.

\section{TABLE I}

SUMMARY OF THE BLADE ROOT LOAD REDUCTION, THE TRAILING EDGE FLAP TRAVEL DISTANCE AND THE MEAN POWER PRODUCTION FOR THE FOUR MPC USED IN THIS PAPER. THE LOAD REDUCTION AND THE POWER LOSS ARE EXPRESSED AS A PERCENTAGE OF THE BASELINE CASE, WHEREAS THE TRAVEL DISTANCE IS RELATIVE TO THE LOW-ORDER FILTER MPC CASE.

\begin{tabular}{c|c|c|c}
\hline & Load Reduction & Travel Distance & Power \\
\hline \hline Low-order filter & $-6.2 \%$ & ref & $-0.3 \%$ \\
High-order filter & $-0.8 \%$ & $+78 \%$ & $-0.4 \%$ \\
Zero-phase filter & $-4.5 \%$ & $+76 \%$ & $-0.4 \%$ \\
DFT & $-7.4 \%$ & $-24 \%$ & $-0.4 \%$ \\
\hline
\end{tabular}

inputs and predicted inputs. The horizon length $N$ has to be large enough so that the predicted inputs represent a fair proportion of the $\mathcal{U}$ vector. All those requirements mean that such a method works well only with either low sampling frequencies, or long horizon length, which may not suit realtime applications.

Table I summarises the load reduction, the trailing edge flap travel distance and the power loss achieved with each of the model predictive controllers.

\section{EXPERIMENTAL RESULTS}

\section{A. Condition of the experiment}

Due to several issues with the test turbine, several sensors and actuators were not working when the tests started. Only the outer trailing edge flap was working, which limited the potential load reduction achievable with the trailing edge flaps. The pitch position sensor had also failed, making the online estimation of the mean free wind speed $\gamma$ impossible. It was instead estimated from the wind speed measurements made on the met mast, and adjusted manually from time to time. Load reduction could however be clearly identified during the tests.

The model predictive control was tested during several hours, splitted in several days with different wind conditions. The protocol chosen was to run tests alternating 2 minutes with
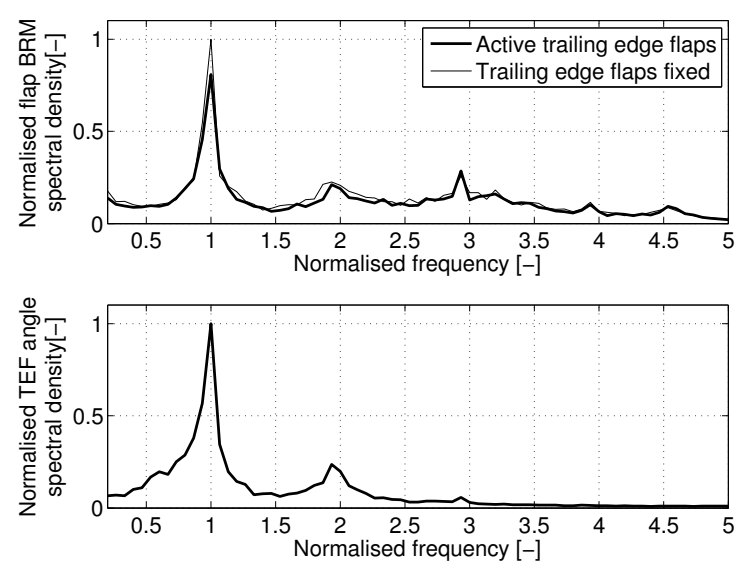

Fig. 10. Experimental results. Measured flapwise blade root moment spectral density (top) and trailing edge flap angle spectral density (bottom), when trailing edge flaps are actuated by the model predictive control (thick line) and when trailing edge flaps are fixed (thin line).

active trailing edge flaps, and 2 minutes with trailing edge flaps fixed in their neutral position. It was estimated that running 2 minute time series was long enough to see the impact of the trailing edge flaps, and short enough to have similar wind conditions for consecutive tests, and thus be able to compare the results when flaps are active or not.

Only the model predictive control with costs on zero-phase filtered inputs and outputs as described in section VI-B was tested.

\section{B. Results}

Here are presented results from a 38 minute test, consisting in 10 2-minute tests with active trailing edge flaps, alternating with 9 2-minute tests with trailing edge flaps fixed in their neutral position, at a wind speed of $10 \mathrm{~m} \cdot \mathrm{s}^{-1}$.

Fig. 10 shows the power spectral density of the flapwise blade root moment and of the trailing edge flap measured position, for the tests when trailing edge flaps were controlled and when they were fixed in their neutral position. Each power spectral density is the average of all the power spectral densities of each 2-minute time series, when flaps are either active of fixed. Those power spectral densities show a clear reduction of the loads at the $1 \mathrm{P}$ and $2 \mathrm{P}$ frequencies, and an actuation of the trailing edge flaps at the same frequencies, and almost only at those frequencies. Fig. 11 shows a short time series of the blade root flapwise moment, and of the trailing edge flap target signal. The target signal to the TEF low-level controller is a smooth signal, without noise or high frequencies targets which would be unefficient from a load response point of view, and which would damage the actuators. Such a result is achieved without adding any low-pass or bandpass filters on the strain gauge signals or before sending the trailing edge flap target to the TEF low level controller, but just by the use of high-order zero-phase filters in the cost function of the model predictive control, and of the adequately tuned Kalman filter.

The vertical dashed lines in Fig. 11 show points where the reference input (trailing edge flap target) derivative is 

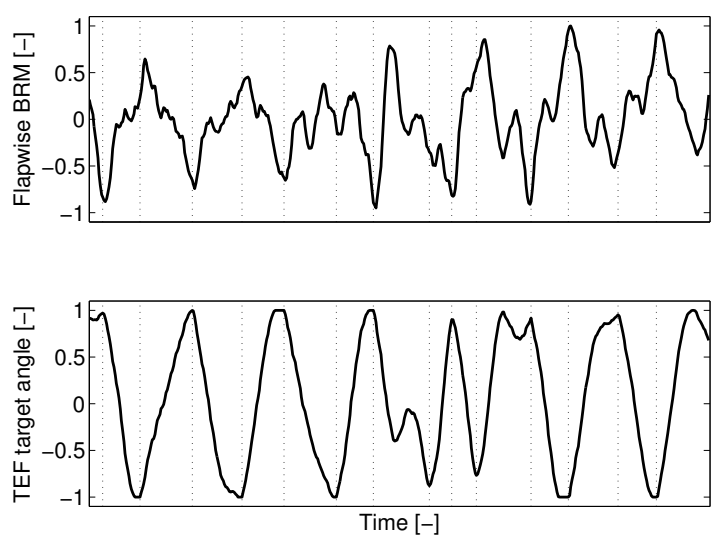

Fig. 11. Experimental results. Time series of the flapwise blade root moment (top) and of the trailing edge flap target (bottom) when the trailing edge flap is controlled by the model predictive control. The vertical dashed lines show points where the trailing edge flap target derivative is 0 .

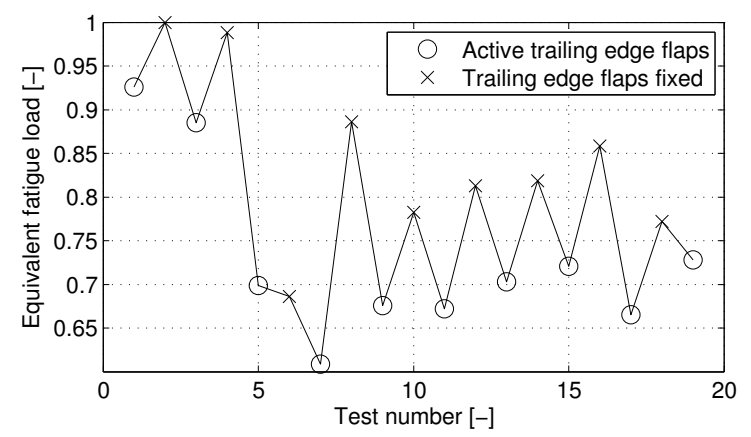

Fig. 12. Experimental results. Normalised flapwise blade root equivalent fatigue loads of the 192 minute time series.

0. They show that, most of the time, the trailing edge flap target reacts ahead of time. For example, the trailing edge flap target starts decreasing a few time steps before the actual blade root moment reaches its minimum, making the controller more efficient. This is due to the prediction of the $1 \mathrm{P}$ and $2 \mathrm{P}$ disturbance states in the model predictive control.

In terms of fatigue load, an average reduction of $-13.8 \%$ of the flapwise blade root fatigue loads is measured. Blade equivalent fatigue loads are very sensitive to single events with high amplitude, like a wind gust or a large wind shear, which would make the comparison between the time series difficult. Nevertheless, plotting the equivalent flapwise blade root fatigue load for each of the 2 minute-time series shows that the model predictive control consistently reduce the blade root loads (Fig. 12).

Fig. 13 shows the time series of, from top to bottom, the blade root flapwise moment, the $1 \mathrm{P}$ disturbance state, the $2 \mathrm{P}$ disturbance state, the quasi-steady disturbance state and the controller input reference. All the data are normalised, except for the disturbance states. The $1 \mathrm{P}$ and $2 \mathrm{P}$ disturbance states are meant to capture the $1 \mathrm{P}$ and $2 \mathrm{P}$ components of the wind speed seen by the rotating blade. Fig. 13 shows that the $1 \mathrm{P}$ and the $2 \mathrm{P}$ content of the blade root flapwise moment are translated into respectively the $1 \mathrm{P}$ and the $2 \mathrm{P}$ disturbance states. Those $1 \mathrm{P}$
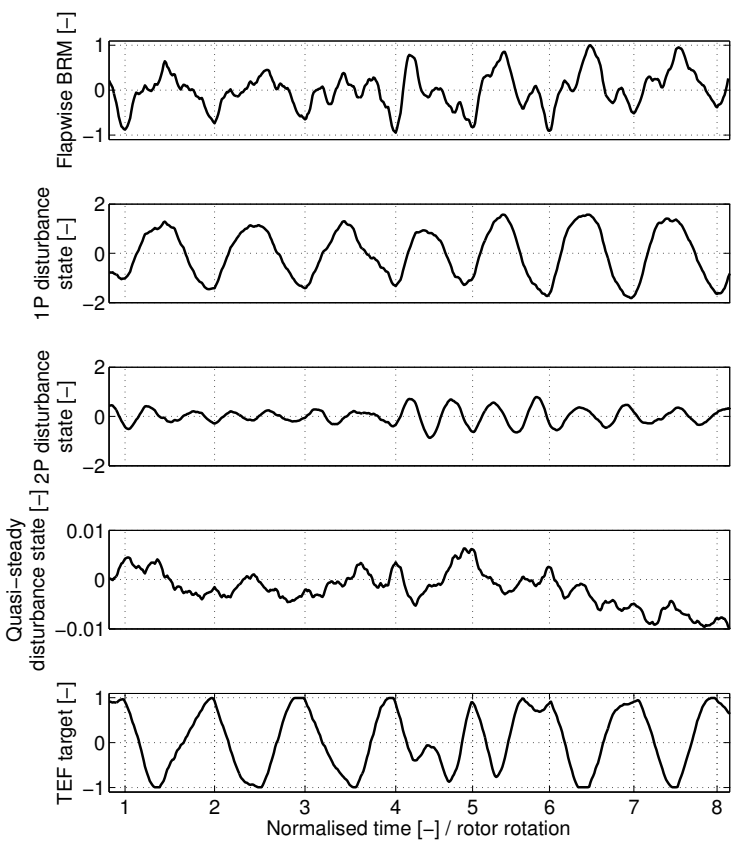

Fig. 13. Experimental results. Time series of, from top to bottom, the normalised flapwise blade root moment, the $1 \mathrm{P}$ and $2 \mathrm{P}$ disturbance states, the quasi-steady disturbance state and the controller input reference. those time series are recorded when the model predictive control is run on the V27 wind turbine.

and $2 \mathrm{P}$ measured disturbance states are signals with a clear $1 \mathrm{P}$ and $2 \mathrm{P}$ frequency, and which phase and amplitude is linked to the measured blade root flapwise moment. The quasi-steady state purpose is to capture any mismatch between the real wind turbine, and the model of the blade aggregated with the $1 \mathrm{P}$ and $2 \mathrm{P}$ disturbances. Between the third and the fourth rotation, it can be noticed that the quasi-steady state captures some $3 \mathrm{P}$ signals because there were no $3 \mathrm{P}$ disturbance state in this model. The trailing edge flap target is based on what is captured by the design model: the blade states and the 1P and $2 \mathrm{P}$ disturbances.

\section{CONCLUSION}

This paper showed the results from a full-scale test of trailing edge flaps on the Vestas V27 wind turbine. Even thought only $5 \%$ of the blade span was covered with a working trailing edge flap, clear load reduction could be observed. $13.8 \%$ flapwise blade root fatigue load reduction was achieved on a 38 minute test. The study of the flapwise blade root moment spectral densities shows a clear reduction of the $1 \mathrm{P}$ and $2 \mathrm{P}$ loads.

The trailing edge flap controller tested on the demonstrator turbine was a frequency-weighted model predictive control with costs on zero-phase filtered flapwise blade root moment and trailing egde flap deflection. Simulations showed that the use of conventional high-order filters in the cost function could introduce large time lags in the system, resulting in an increase of the blade root loads. This loads increase does not happen when zero-phase filters are used, or when the costs are applied 
to the amplitude of the Fourier coefficients of the system inputs and outputs.

\section{REFERENCES}

[1] "IEC 61400-1 Ed3: Wind turbines - part 1: Design requirements." ISBN: 978-2-88912-201-1.

[2] T. Burton, D. Sharpe, N. Jenkins, and E. A. Bossanyi, Wind Energy handbook. Wiley, 2008.

[3] E. A. Bossanyi, "Individual blade pitch control for load reduction," Wind Energy, vol. 6, no. 2, pp. 119-128, 2003. [Online]. Available: http://dx.doi.org/10.1002/we.76

[4] - "Further load reductions with individual pitch control," Wind Energy, vol. 8, no. 4, pp. 481-485, 2005. [Online]. Available: http://dx.doi.org/10.1002/we.166

[5] T. J. Larsen, H. A. Madsen, and K. Thomsen, "Active load reduction using individual pitch, based on local blade flow measurements," Wind Energy, vol. 8, no. 1, pp. 67-80, 2005. [Online]. Available: http://dx.doi.org/10.1002/we.141

[6] K. Selvam, S. Kanev, J. W. van Wingerden, T. van Engelen, and M. Verhaegen, "Feedback-feedforward individual pitch control for wind turbine load reduction," International Journal of Robust and Nonlinear Control, vol. 19, no. 1, pp. 72-91, 2009. [Online]. Available: http://dx.doi.org/10.1002/rnc.1324

[7] M. H. Hansen, A. Hansen, T. J. Larsen, S. Øye, P. Sørensen, and P. Fuglsang, "Control design for a pitch-regulated, variable speed wind turbine," Ris $\varnothing$ National Laboratory, Tech. Rep. Risø-r-1500(EN), January 2005.

[8] T. D. Ashwill, G. Kanaby, K. Jackson, and M. Zutech, "Development of the swept twist adaptive rotor (star) blade," in Proceedings of the 48th AIAA Aerospace Sciences Meeting Including the New Horizons Forum and Aerospace Exposition, Orlando, Florida, 2010.

[9] M. H. Hansen, "Aeroelastic modal analysis of backward swept blades using HAWCStab2," Ris $\varnothing$ DTU, National Laboratory for Sustainable Energy, Tech. Rep. Ris $\varnothing-R-1769$ (EN), February 2011.

[10] J. A. Paquette and P. S. Veers, "Increased rotor size through passive load control and weight reduction concepts," in EWEC 2009, Marseille, France, 2009.

[11] M. Capellaro and M. Kühn, "Boundaries of bend twist coupling," in TORQUE 2010: The Science of Making Torque from Wind, 2010.

[12] T. Barlas and G. van Kuik, "Review of state of the art in smart rotor control research for wind turbines." Progress in Aerospace Sciences, vol. 46, no. 1, pp. 1-27, 2010.

[13] T. Behrens and W. Jun Zhu, "Feasibility of aerodynamic flap hinge moment measurements as input for load alleviation control," in EWEA 2011, Brussels, Belgium, 2011.

[14] S. Basualdo, "Load alleviation on wind turbine blades using variable airfoil geometry," Wind Engineering, vol. 29, no. 2, pp. 169-182, Mar. 2005.

[15] T. Buhl, M. Gaunaa, and C. Bak, "Potential load reduction using airfoils with variable trailing edge geometry," Journal of Solar Energy Engineering, vol. 127(4), pp. 503-516, November 2005.

[16] P. B. Andersen, L. Henriksen, M. Gaunaa, C. Bak, and T. Buhl, "Deformable trailing edge flaps for modern megawatt wind turbine controllers using strain gauge sensors," Wind Energy, vol. 13, no. 2-3, pp. 193-206, 2010. [Online]. Available: http://dx.doi.org/10.1002/we.371

[17] D. Castaignet, T. Buhl, N. K. Poulsen, and J. J. Wedel-Heinen, "Trailing edge flaps impact on fatigue and extreme loads in power production." in EWEA 2011, Brussels, Belgium, 2011.

[18] C. Bak, M. Gaunaa, P. B. Andersen, T. Buhl, P. Hansen, and K. Clemmensen, "Wind tunnel test on airfoil Ris $\varnothing-\mathrm{B} 1-18$ with an active trailing edge flap," Wind Energy, vol. 13, no. 2-3, pp. 207-219, 2010. [Online]. Available: http://dx.doi.org/10.1002/we.369

[19] J. W. van Wingerden, A. W. Hulskamp, T. Barlas, B. Marrant, G. A. M. van Kuik, D.-P. Molenaar, and M. Verhaegen, "On the proof of concept of a smart wind turbine rotor blade for load alleviation," Wind Energy, vol. 11, no. 3, pp. 265-280, 2008. [Online]. Available: http://dx.doi.org/10.1002/we.264

[20] P. B. Andersen, C. Bak, M. Gaunaa, and T. Buhl, "Wind tunnel test of a closed loop controller for an airfoil with trailing edge flaps," in TOROUE 2010: The Science of Making Torque from Wind, Crete, Greece, June 2010.

[21] J.-W. van Wingerden, A. Hulskamp, T. Barlas, I. Houtzager, H. Bersee, G. van Kuik, and M. Verhaegen, "Two-degree-of-freedom active vibration control of a prototyped smart rotor," Control Systems Technology, IEEE Transactions on, vol. 19, no. 2, pp. 284 -296, march 2011.
[22] D. Castaignet, J. J. Wedel-Heinen, T. Kim, T. Buhl, and N. K. Poulsen, "Results from the first full scale wind turbine equipped with trailing edge flaps," in 28th AIAA Applied Aerodynamics Conference, Chicago, Illinois, June 2010.

[23] M. A. Lackner and G. van Kuik, "A comparison of smart rotor control approaches using trailing edge flaps and individual pitch control," Wind Energy, vol. 13, no. 2-3, pp. 117-134, 2010. [Online]. Available: http://dx.doi.org/10.1002/we.353

[24] J. K. Rice and M. Verhaegen, "Robust and distributed control of a smart blade," Wind Energy, vol. 13, no. 2-3, pp. 103-116, 2010. [Online]. Available: http://dx.doi.org/10.1002/we.362

[25] D. G. Wilson, D. E. Berg, M. F. Barone, J. C. Berg, B. R. Resor, and D. W. Lobitz, "Active aerodynamic blade control design for load reduction on large wind turbines," in EWEC 2009, Marseille, France, March 2009.

[26] D. Q. Mayne, J. B. Rawlings, C. V. Rao, and P. O. M. Scokaert, "Constrained model predictive control: Stability and optimality," Automatica, vol. 36, pp. 789-814, 2000.

[27] M. O. L. Hansen, Aerodynamics of Wind Turbines. Earthscan, 2008, ISBN: 978-1-84407-438-9.

[28] P. B. Andersen, M. Gaunaa, C. Bak, and M. H. Hansen, "A dynamic stall model for airfoils with deformable trailing edges," Wind Energy, vol. 12, no. 8, pp. 734-751, 2009. [Online]. Available: http://dx.doi.org/10.1002/we.326

[29] J. M. Maciejowski, Predictive Control with Constraints. Prentice-Hall, 2002.

[30] qpOASES homepage. Accessed 26/10/2011. [Online]. Available: http://www.kuleuven.be/optec/software/qpOASES

[31] H. J. Ferreau, H. G. Bock, and M. Diehl, "An online active set strategy to overcome the limitations of explicit mpc," International Journal of Robust and Nonlinear Control, vol. 18, no. 8, pp. 816-830, 2008. [Online]. Available: http://dx.doi.org/10.1002/rnc.1251

[32] D. Castaignet, N. K. Poulsen, T. Buhl, and J. J. Wedel-Heinen, "Model predictive control of trailing edge flaps on a wind turbine blade," in American Control Conference, 2011.

[33] CBLAS homepage. Accessed 05/12/2011. [Online]. Available: http://www.netlib.org/blas/

[34] F. Gustafsson, "Determining the initial states in forward-backward filtering," IEEE Transactions on Signal Processing, vol. 44, no. 4, pp. 988-992, Apr. 1996.

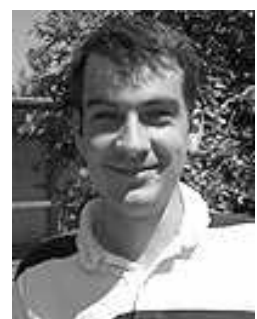

Damien Castaignet received a M.Sc. degree in Engineering from École Centrale Paris, France and a M.Sc. degree in Engineering Physics from KTH, the Royal Institue of Technology, Stockholm, Sweden. $\mathrm{He}$ is currently employed by Vestas Wind Systems and is pursuing a Ph.D. degree in the Wind Energy department of Ris $\varnothing$ DTU, Technical University of Denmark, Lyngby, Denmark. His focus is on the control of trailing edge flaps for active load reduction

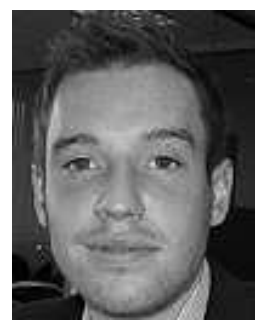

Ian Couchman received his undergraduate degree from the University of Cambridge in 2006. $\mathrm{He}$ finished his $\mathrm{Ph} . \mathrm{D}$. on control of fluid mixing at Imperial College London in 2010 and now works for the Global Research department of Vestas. His research interests include wind energy applications, large-scale systems and model reduction. 


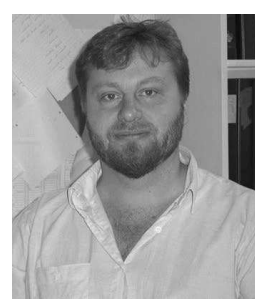

Niels Kjølstad Poulsen was born in the central part of Sjælland, Denmark in 1956. He received his M.Sc. and Ph.D. degrees in electrical engineering from The Institute of Mathematical Statistics and Operations Research (IMSOR), the Technical University of Denmark, in 1981 and 1984, respectively. $\mathrm{He}$ has been employed at the Technical university of Denmark from 1984, since 1990 as an associate professor at The Department of Informatics and Mathematical Modelling. His primary research identification and fault diagnosis.

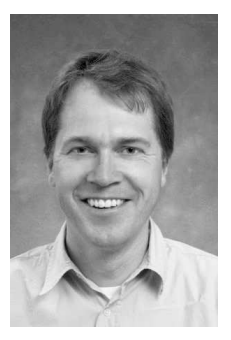

Jens-Jakob Wedel-Heinen is a Maritime Engineer and $\mathrm{PhD}$ from the Technical University of Denmark. $\mathrm{He}$ has an extensive background in certification of wind turbines for Det Norske Veritas. $\mathrm{He}$ is a specialist in wind turbine load assessment as well as design, manufacturing and testing of blades and steel structures. Since 2008 he has been a manager for Vestas Wind Systems and a project manager for the ATEF project sponsored by the Danish National Advanced Technology Fund on world's first demonstration of flaps on a full scale wind turbine.

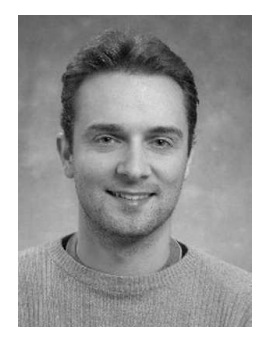

Thomas Buhl is Head of Wind turbine structures and Head of Offshore Wind Energy at Risø DTU. He has been Scientist and Senior scientist at Ris $\varnothing$ DTU the previous nine years within aeroelasticity. $\mathrm{He}$ holds a PhD and a MSc in structural optimization. 


\section{Paper C}

Trailing edge flaps impact on fatigue and extreme loads in power production

Paper published in the procedeedings of the EWEA conference, March 14 - 17, 2011, Brussels, Belgium. 


\title{
Trailing edge flaps impact on fatigue and extreme loads in power production.
}

\author{
Damien Castaignet, Thomas Buhl, Niels K. Poulsen and Jens Jakob Wedel-Heinen
}

\begin{abstract}
A Model Predictive Control is designed to alleviate fatigue loads with trailing edge flaps. The controller is run on a generic multi-megawatt wind turbine, for all the power production load cases as defined in the IEC $61400-1$, ed3 standards. The robustness of the controller with respect to the first blade eigenfrequency and the trailing edge flap efficiency is verified.

Fatigue loads are decreased in the main components of the turbine. Blade root flapwise fatigue loads, which are the only ones explicitely targeted by the controller, are reduced by $22.8 \%$. The main bearing and the tower also benefit largely from the trailing edge flaps: $32 \%$ fatigue load reduction in the main bearing tilt and yaw moments, and $23 \%$ at the tower/nacelle interface. Extreme loads during normal production are also decreased, but in a smaller proportion: only $7 \%$ are reached at the blade root, and between $5 \%$ and $10 \%$ at the main bearing. Extreme loads happening during load cases others than normal production have not been taken into account, and would modify those results.

Simulations of the normal production load cases showed two major drawbacks: the annual energy production of the turbine is decreased, and the travel distance of the flaps reach several billion degrees over 20 years. This is a reliability challenge for such actuators. Trailing edge flaps can be economically viable for wind turbine manufacturers only if those issues are solved.
\end{abstract}

\section{INTRODUCTION}

Trailing edge flaps (TEF), microtabs and other local aerodynamic control surfaces have been extensively studied in order to achieve blade fatigue loads reduction [1]-[7]. Depending on the actuator size, deflection and speed, up to $60 \%$ decrease in blade root flapwise fatigue loads were achieved on simulations.

Load reduction at the blade root induces fatigue load reduction in other wind turbine

This work is part of the ATEF project, which is partially funded by the Danish National Advanced Technology Foundation (Højteknologifonden)

D. Castaignet and J.J. Wedel-Heinen are with Vestas Wind Systems A/S, Global Research, Roskilde, Denmark dacalvestas.com

T. Buhl is with the Wind Energy Department, Rise DTU National Laboratory for Sustainable Energy, Roskilde, Denmark

N. K. Poulsen is with DTU Informatics, Technical University of Denmark, Lyngby, Denmark components like the tower and the main shaft [3]. Berg and al. initial studies on a 1.5MW gearbox model showed that gearbox fatigue loads could be reduced by the use of "active aero load control" [8].

The ultimate goal of using smart rotors is to be able to reduce the cost of energy. Wind turbines are now optimised for both fatigue and extreme loads according to IEC standards [9]. A significant reduction of the cost of wind energy can be achieved with smart rotors only if they are able to tackle both fatigue and extreme loads.

Lackner and al. [10] started investigating the use of smart rotors to reduce extreme loads. The authors use a controller designed for fatigue load reduction, and test it during simple step function in wind speeds, both locally and globally.

In this paper, all the power production load cases of the IEC $61400-1$, ed 3 standards are run with a generic megawatt wind turbine equipped with trailing edge flaps. The trailing edge flaps controller is designed explicitly for blade root flapwise fatigue loads only. But both fatigue and extreme loads in the blades, the main bearing and the tower, as well as the power production, are studied.

\section{SYStem / SetuP}

\section{A. Turbine}

Simulations are run with the model of a generic multi-megawatt turbine. The turbine is an upwind, pitch controlled, variable-speed, three bladed, horizontal-axis turbine. One single trailing edge flap is mounted on each of the 3 blades, towards the tip of the blade. The original pitch controller is unchanged, there is no communication between the pitch controller and the trailing edge flaps controller. The only sensors available to the trailing edge flap controller are the flapwise blade root moment of each blade, and an estimated mean wind speed used for gain scheduling of the controller. 
TABLE ।

Simulated load cases, based on the IEC 61400-1, ed3 standards [9]

\begin{tabular}{|c|l|}
\hline 1.1 & Power production (fatigue analysis) \\
\hline 1.2 & $\begin{array}{l}\text { Power production (ultimate strength } \\
\text { analysis) }\end{array}$ \\
\hline 1.3 & $\begin{array}{l}\text { Power production with extreme turbu- } \\
\text { lence model (ultimate strength analysis) }\end{array}$ \\
\hline 1.4 & $\begin{array}{l}\text { Power production with extreme coher- } \\
\text { ent gust with direction change (ultimate } \\
\text { strength analysis) }\end{array}$ \\
\hline 1.5 & $\begin{array}{l}\text { Power production with extreme wind } \\
\text { shear (ultimate strength analysis) }\end{array}$ \\
\hline 2.1 & $\begin{array}{l}\text { Power production plus occurence of } \\
\text { fault (ultimate strength analysis) }\end{array}$ \\
2.2
\end{tabular}

\section{B. Load cases}

Simulations are run for load cases based on the IEC 61400, ed3 standards [9]. Load cases related to idling and parked conditions are not run as no trailing edge flaps controllers were designed for this purpose. Table I summarises the load cases simulated to calculate the fatigue and the extreme loads in this paper.

Fatigue loads over 20 years are calculated for all relevant components: blades, main bearing and tower (Table II). The rainflow counting method counts the number of cycles $N_{i}$ at a given stress range $\sigma_{i}$ the material is going throught. The Wöhler curve enables to calculate an equivalent stress range $\sigma^{e q}$ which is representative of the damage of the material after 20 years:

$$
\sigma^{e q}=\sum_{i} \sigma_{i}\left(\frac{N_{i}}{N^{e q}}\right)^{1 / m}
$$

The damage resulting from the $N_{i}$ cycles at the stress ranges $\sigma_{i}$ is equivalent to $N^{e q}$ cycles at a stress range $\sigma^{e q} . m$ is a constant specific to the material.

Extreme loads over 20 years are calculated for the same components. A safety factor as described in the standards is used.

\section{Simulation code}

Numerical analysis is carried out with the aeroelastic code Flex5, developed by DTU Mechanical Engineering. It is a state-of-the art Blade Element Momentum code based on the modal approach. Both blades and tower are flexible, modeled by as many mode shapes as required. It has all the usual engineering models used in the wind turbine simulation tools, such as Prandtl-Glauert tip correction, dynamic wake model, turbulent wind, oblique
TABLE ॥

Investigated loads: fatigue and extreme loads in the blade, the main bearing and the tower are investigated.

\begin{tabular}{|c|c|}
\hline $\mathrm{Mx0}, \mathrm{My0}$ & $\begin{array}{l}\text { Blade root flapwise }(\mathrm{MxO}) \text { and edge- } \\
\text { wise (My0) moment }\end{array}$ \\
\hline Mx3/4, My3/4 & $\begin{array}{l}\text { Blade flapwise }(\mathrm{M} \times 3 / 4) \text { and edge- } \\
\text { wise (My3/4) moments, at a radius } \\
\text { of } 3 / 4 \text { the blade length }\end{array}$ \\
\hline MxMB & Main bearing tilt moment \\
\hline MzMB & Main bearing yaw moment \\
\hline MyMB & Main bearing driving moment \\
\hline MxTT & $\begin{array}{l}\text { Tilt moment at the tower/nacelle in- } \\
\text { terface }\end{array}$ \\
\hline MzTT & $\begin{array}{l}\text { Yaw moment at the tower/nacelle } \\
\text { interface }\end{array}$ \\
\hline $\mathrm{MbT}$ & $\begin{array}{l}\text { Equivalent tower moment at founda- } \\
\text { tion level }\end{array}$ \\
\hline
\end{tabular}

inflow model etc. The dynamic stall model originally coded in Flex5 works only for rigid blade sections [11]. The implementation of the trailing edge flaps aerodynamics in Flex5 is based on the model by Andersen et al. [12].

\section{Controller}

\section{A. Design model}

The controller used in this paper is a linear model predictive control. The design model is based on the non-linear aero-elastic model of a wind turbine developed at DTU Mechanical Engineering and implemented in the aeroelastic code Flex [13]. This non-linear model is simplified and linearised. The number of states in the design model and the potentiality of running the controller in real-time on a wind turbine is an issue.

The blade structure is modeled by its first flap mode: the flapwise and edgewise deflections of the blade section $x$ at time $t$, respectively $u_{z}(x, t)$ and $u_{y}(x, t)$, are

$$
\begin{aligned}
& u_{z}(x, t)=g(t) u_{z}^{1}(x) \\
& u_{y}(x, t)=g(t) u_{y}^{1}(x)
\end{aligned}
$$

$u_{z}^{1}(x)$ and $u_{y}^{1}(x)$ are respectively the flapwise and edgewise deflection of the blade section $x$ under the first flapwise mode shape. $g(t)$ is the generalised coordinate associated to this mode shape.

The generalised force and the blade root flap moment $M_{f}$ are derived as a linear function of the states $g$ and $\dot{g}$, the rotor speed $\omega$, the pitch angle $\varphi$, the trailing edge flap angle $\beta$ and the mean wind speed $V$ [14]. An input disturbance state $d_{i}$ is added to deal with the difference between the design model and the 
real turbine, and a predictive Kalman filter is used to estimate the states.

For each of the blades, the design model is written in the state-space form:

$$
\begin{aligned}
\dot{\mathbf{x}} & =\mathbb{A} \mathbf{x}+\mathbb{B} \mathbf{u}+\mathbb{G} \mathbf{d} \\
\mathbf{y} & =\mathbb{C} \mathbf{x} \\
\mathbf{z} & =\mathbb{C}_{m} \mathbf{x}
\end{aligned}
$$

where the state vector $\mathrm{x}$ is

$$
\mathbf{x}=\left(\begin{array}{c}
\tilde{g} \\
\dot{\tilde{g}} \\
d_{i}
\end{array}\right)
$$

the input vector $\mathbf{u}$ is

$$
\mathbf{u}=\tilde{\beta}
$$

the disturbance vector $\mathbf{d}$ is

$$
\mathbf{d}=(\tilde{\varphi})
$$

the output vector $\mathbf{y}$ is

$$
\mathbf{y}=\left(\tilde{M}_{f}\right)
$$

the measurement vector $\mathbf{z}$ is

$$
\mathbf{z}=\left(\tilde{M}_{f}\right)
$$

The upperscript on a variable indicates the difference between the given variable and its steady-state value.

\section{B. Model Predictive Control}

The model predictive control designed in this paper targets only the fatigue loads: $\sigma^{e q}$ (equation 1) has to be as small as possible. The $m$ constant of glass fibers is around 10-12. So the stress range $\sigma_{i}$ has a higher impact on the damage than the cycles number $N_{i}$ : the flap controller should focus on the high amplitude loads. Loads with a very low frequency, below $1 \mathrm{P}$, should not be targeted: they are the consequence of mean wind speed fluctuation, and reducing their amplitude would result in high power loss. Loads with higher frequencies, above 3P, do not contribute much to the blade damage because of their low amplitude. They would moreover be hard to reduce as they are mainly due to the stochastic turbulence part of the wind which is very hard to predict, and because the non steady aerodynamics which are not modeled in the design model become more important.

The model predictive controller is then designed to target the loads which have a frequency between $1 \mathrm{P}$ and $3 \mathrm{P}$. The cost function minimised by the model predictive control is not a function of the blade root flap moment
$\left(M_{f}\right)$, but a bandpass filtered blade root flap moment $(\breve{y})$ [14].

A cost is also added on a high-pass filtered trailing edge flap angle $\breve{u}$ in order to penalise the use of the flaps at high frequencies, where they will be inefficient and they would be worn down quickly.

Finally a cost on the trailing edge flap position ensures that the flap mean position is close to 0 .

The cost function, over $N_{\text {hor }}$ horizon steps, is:

$$
\phi=\sum_{i=1}^{N_{\text {hor }}}\left\|\breve{y}_{i}\right\|_{W_{\breve{y}}}^{2}+\sum_{i=1}^{N_{\text {hor }}}\left\|u_{i}\right\|_{W_{u}}^{2}+\sum_{i=1}^{N_{\text {hor }}}\left\|\breve{u}_{i}\right\|_{W_{\breve{u}}}^{2}
$$

where $\left\|\breve{y}_{i}\right\|_{W_{\breve{y}}}^{2}=\breve{y}_{i}^{T} W_{\breve{y}} \breve{y}_{i} . W_{\breve{y}}, W_{u}$ and $W_{\breve{u}}$ are the weight factors on respectively the bandpass filtered blade root flap moment $(\breve{y})$, the trailing edge flap deflection $(u)$ and the high pass filtered trailing edge flap deflection $(\breve{u})$.

Hard constraints are added on the trailing edge flap deflection and deflection rate. No hard or soft constraints are added on the states: extreme loads and tower clearance are not targeted by this controller.

\section{Results}

Simulations are run to check the performance of the controller, its robustness with respect to the blade eigenfrequencies and the trailing edge flap efficiency, and the potential benefits of having trailing edge flaps assuming that flaps would make the blade $10 \%$ heavier. For this purpose, the following wind turbines are modeled:

- WT0 the baseline wind turbine

- WT- 5 with a blade mass decreased by $5 \%$

- WT+5 with a blade mass increased by $5 \%$

- WT+10 with a blade mass increased by $10 \%$

Three different controller configurations are tested:

- MPC0 the baseline model predictive controller

- MPC+ where the trailing edge flap efficiency is overestimated by $50 \%$

- MPC- where the trailing edge flap efficiency is underestimated by $50 \%$

The trailing edge flap efficiency is explained in section IV-B.2. 


\section{A. Baseline}

Loads on the baseline turbine WTO without trailing edge flaps are compared with loads on the same turbine with the baseline control MPCO (Table III).

An overall reduction of $23 \%$ on the flapwise blade root fatigue loads is achieved. They are the only loads targeted directly by the controller, but other loads also benefit from the trailing edge flap controller. The extreme flap moment is reduced by $7 \%$.

Blade root edgewise loads were expected to increase due to the increase of drag. But both fatigue and extreme blade root edgewise loads are actually decreased. Most of the load reduction happens at a frequency close to the eigenfrequency of a wind turbine mode which combines flapwise and edgewise blade modes. The reduction in flapwise blade mode is high enough to lead to a reduction in edgewise blade root moment as well, despite the drag increase due to the trailing edge flap actuation.

The main bearing also benefits from the trailing edge flap controller: the yaw and tilt fatigue loads are decreased by more than $30 \%$, whereas their extreme loads are decreased by 5 to $10 \%$. This load reduction in the main bearing transfers to the tower, where fatigue loads are reduced by 10 to $25 \%$, and extreme loads by 5 to $15 \%$.

The fatigue load reduction obtained with the trailing edge flap configuration of this paper is similar to the one obtained by Larsen and al. [15] with Individual Pitch Control.

However, two loads are increased: the main bearing driving moment and the flapwise and edgewise moments towards the tip of the blade. The load incease occurs at frequencies above the 3P frequency. Part of the load increase is due to the excitation of the blade second flap mode. This mode can not be targeted by the model predictive control as it's not part of the design model. It could be added to the design model, but an extra strain gauge would be necessary so that the system remains observable. Several independent flaps along the blade would also help damping the blade second flap mode.

Several studies had shown that trailing edge flaps efficiently reduce fatigue loads in the turbine [2]-[4], [16]. Running the load cases defined in the IEC standards showed that extreme loads also benefit from the trailing edge flaps, even if the controller is designed to reduce blade fatigue loads only. The extreme load reduction is nevertheless signifi-
TABLE III

Baseline test: fatigue and extreme load reduction due to the trailing edge flaps.

\begin{tabular}{|l|c|c|c|}
\hline & Sensor & $\begin{array}{c}\text { Fatigue } \\
{[\%]}\end{array}$ & $\begin{array}{c}\text { Extreme } \\
{[\%]}\end{array}$ \\
\hline \multirow{4}{*}{ Blade } & Mx0 (flap) & -22.8 & -6.8 \\
& My0 (edge) & -3.0 & -4.0 \\
& Mx3/4 (flap) & +2.1 & -2.8 \\
& My3/4 (edge) & +6.9 & +6.8 \\
\hline \multirow{3}{*}{ Bearing } & MxMB (tilt) & -32.1 & -11.0 \\
& MzMB (yaw) & -32.2 & -4.7 \\
& MyMB (driving) & +0.7 & +2.2 \\
\hline \multirow{3}{*}{ Tower } & MxTT (tilt,top) & -23.0 & -6.9 \\
& MzTT (yaw,top) & -24.4 & -10.2 \\
& MbT (bottom) & -11.7 & -17.5 \\
\hline
\end{tabular}

cantly lower than the fatigue load reduction. Trailing edge flaps will be implemented by wind turbine manufacturers only if they allow a cost of energy reduction. Whether the load reduction achieved with this controller allows a potential saving on the component manufacturing depends on each turbine specifications, and on the design criteria for each component: fatigue and/or extreme loads. Assuming that the design criteron of the modeled blade is the extreme loads, then such a controller would not result in a significant cost of energy reduction. And the high fatigue load reduction achieved would not result in significant cost of energy reduction. Having specific controllers to target the extreme loads would probably enable a higher impact of the trailing edge flaps on the extreme loads.

\section{B. Robustness}

The performance of the controller depends highly on its tuning and on the design model used. A bad tuning of the Kalman filter for example may result in very high loads [14]. It is thus important to check the robustness of the control, and to make sure that its performance is not affected by the differences between the controller design model and the real turbine.

Two important blade parameters which may be wrongly estimated are the blade first flapwise eigenfrequency and the trailing edge flap efficiency.

1) Blade eigenfrequencies: The eigenfrequencies of the real blade may differ from the ones used in the controller design model. The robustness of the controller towards this parameter is checked by running the baseline controller MPCO with wind turbine models where the blade mass has been increased $(\mathrm{WT}+5)$ and decreased (WT-5) by $5 \%$. The 

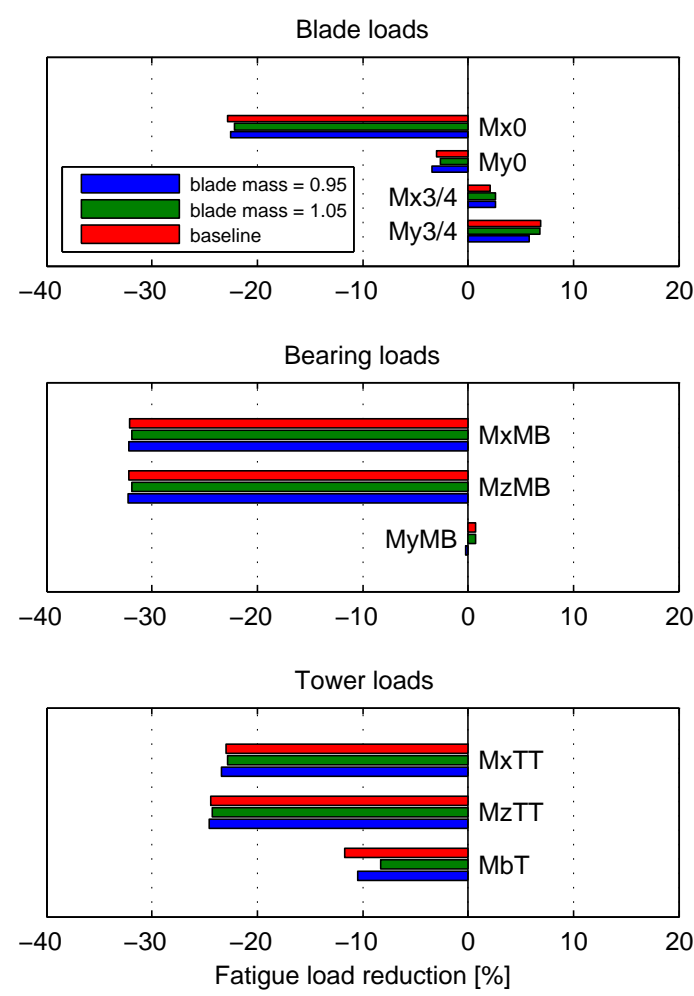

Fig. 1. Robustness with respect to the blade mass. Comparison of the fatigue load reduction when the wind turbine blades have the same mass as in the design model (red) or are $5 \%$ heavier (green) or $5 \%$ lighter (blue).

first flapwise eigenfrequencies of the blade are respectively $2.4 \%$ higher and $2.6 \%$ lower than the eigenfrequency of the baseline turbine. Loads are compared to the loads on the same turbines with the altered blade mass (WT-5 and WT+5), but without trailing edge flaps. Fatigue and extreme loads show the same trend in those both cases as in the baseline case (Figures 1 and 2). None of the loads are significantly increased due to the eigenfrequency modification. The controller is robust towards an uncertainty on the blade first flapwise eigenfrequency up to at least $+/-5 \%$.

2) TEF efficiency: In the controller design model, the lift coefficient $C_{L}$ is modeled as a linear function of the trailing edge flap angle $\beta$. $\frac{\partial C_{L}}{\partial \beta}$ is a constant estimated by means of 2D aerodynamic simulations (XFoil, CFD...) or wind tunnel measurements. Several aspects which would influence the gradient $\frac{\partial C_{L}}{\partial \beta}$ are not taken into account either by the $2 \mathrm{D}$ aerodynamic simulations or the wind tunnel measurements: for example, 3D effects around the flaps or deformation of the trailing edge flaps
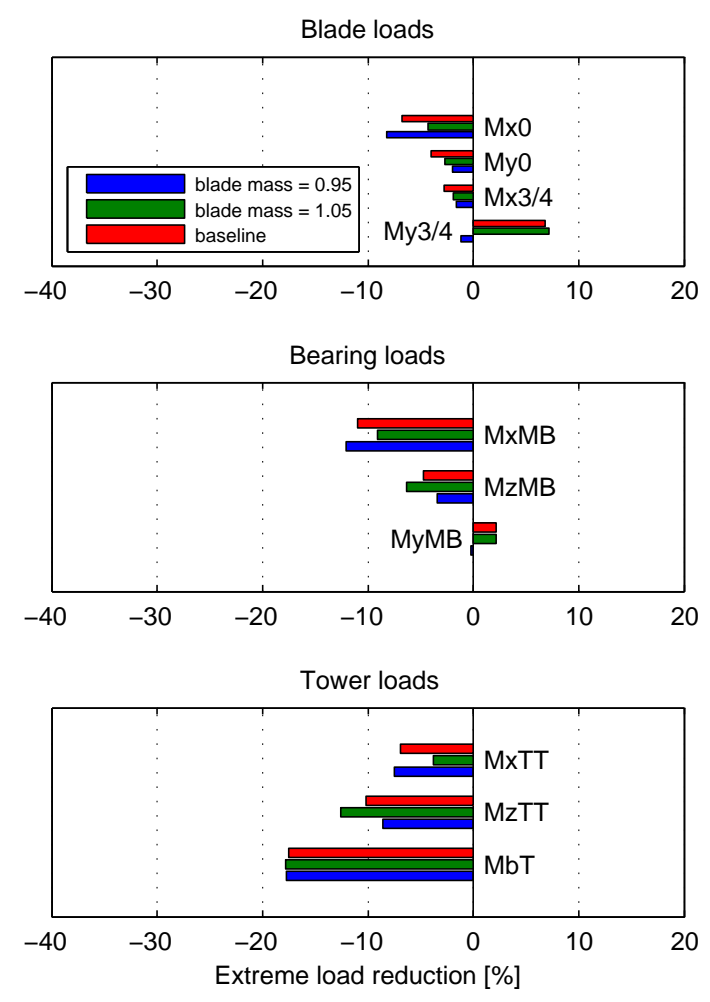

Fig. 2. Robustness with respect to the blade mass. Comparison of the extreme load reduction when the wind turbine blades have the same mass as in the design model (red) or are $5 \%$ heavier (green) or $5 \%$ lighter (blue).

under aerodynamic loads. An extra parameter, the trailing edge flap efficiency $\alpha_{T E F}$ is added in the design model in order to simulate the difference in the gradient $\frac{\partial C_{L}}{\partial \beta}$ between the design model and the real turbine:

$$
\left(\frac{\partial C_{L}}{\partial \beta}\right)_{\text {design }}=\alpha_{T E F}\left(\frac{\partial C_{L}}{\partial \beta}\right)_{2 D}
$$

Simulations are run with a controller designed with $\alpha_{T E F}=1.5$ and with $\alpha_{T E F}=0.5$, which means that the controller respectively overestimates and underestimates the influence of the trailing edge flap angle on the lift coefficient. Those loads are compared to the loads on the baseline turbine, without trailing edge flaps (Figures 3 and 4).

Overestimating the trailing edge flap efficiency results in the excitation of a turbine mode which involves the second flap mode of the blade. As a consequence, main bearing driving moment loads increase slightly and blade loads towards the tip of the blade increase drastically (more than $50 \%$ ). On the contrary, underestimating the trailing edge flap 

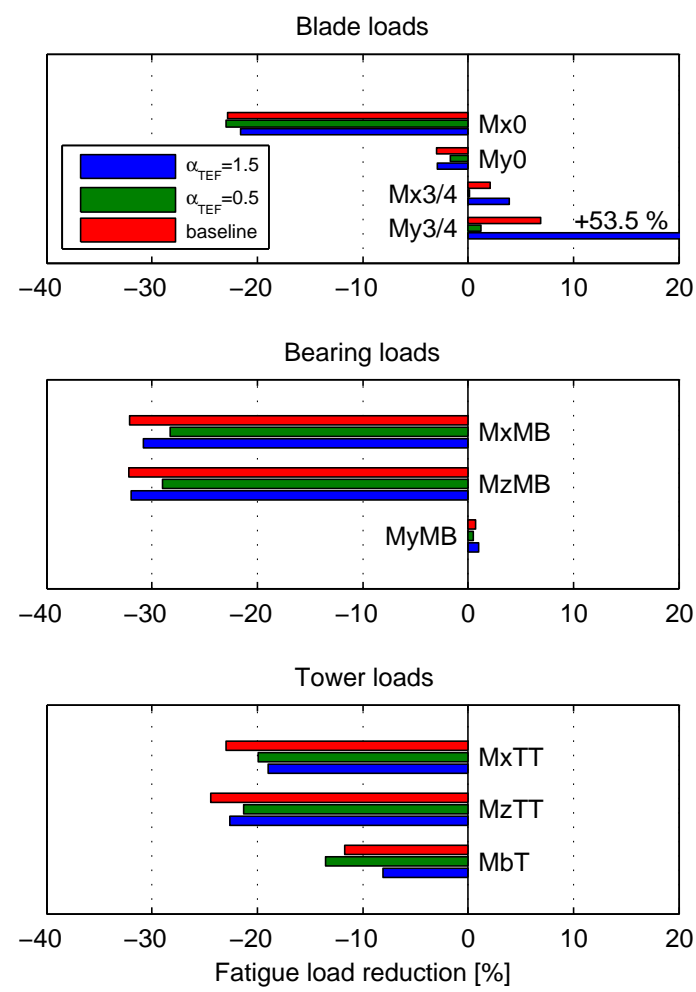

Fig. 3. Robustness with respect to the trailing edge flap efficiency. Comparison of the fatigue load reduction when the trailing edge flaps efficiency, in the design model, are the same as in the simulated turbine (red), overestimated by $50 \%$ (blue) or underestimated by $50 \%$ (green).

efficiency reduces the controller efficiency, but it does not lead to any significant load increase.

Even if the specific model predictive control used in these simulations has not been designed explicitly to be robust, simulations showed that the controller performance were robust towards a $+/-2.5 \%$ blade eigenfrequency uncertainty. The controller performance are also preserved in spite of a $50 \%$ underestimation of the trailing edge flap efficiency. But on the contrary, a $50 \%$ overestimation of the trailing edge flaps efficiency results in excessive loads.

\section{Load reduction considering a $10 \%$ blade mass increase}

One of the benefits from having trailing edge flaps on a blade would be to decrease the quantity of reinforcement (glass or carbon fibers) used in the blades as fatigue and extreme loads are decreased. On the other hand, flaps, actuators, sensors and cables add
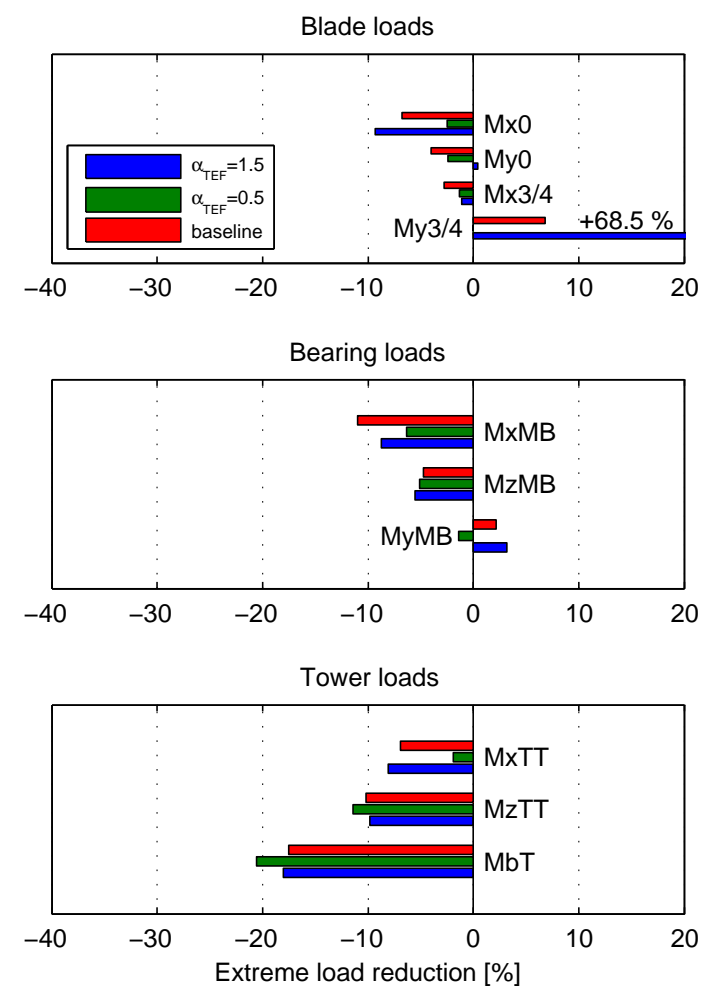

Fig. 4. Robustness with respect to the trailing edge flap efficiency. Comparison of the extreme load reduction when the trailing edge flaps efficiency, in the design model, are the same as in the simulated turbine (red), overestimated by $50 \%$ (blue) or underestimated by $50 \%$ (green).

weigth to the blade. What would happen if the overall blade mass was increased by $10 \%$ ?

Results show that the benefits of the trailing edge flaps on most of the loads are decreased by a few percent, that the blade edgewise loads are even increased compared to the baseline test without trailing edge flaps (Figures 5 and 6 ).

The increase of the extreme tilt moment at the tower/nacelle interface is a logical consequence of the increase of the rotor mass. The nacelle mass distribution is optimised for the baseline rotor, and should be modified in order to fit the new rotor mass. The decrease in fatigue loads at the tower/nacelle interface proves that even if the blades are heavier, the trailing edge flaps still helps reducing the loads at the tower/nacelle interface.

\section{Drawbacks}

1) Power: The trailing edge flap controller is designed only for blade root flapwise load re- 

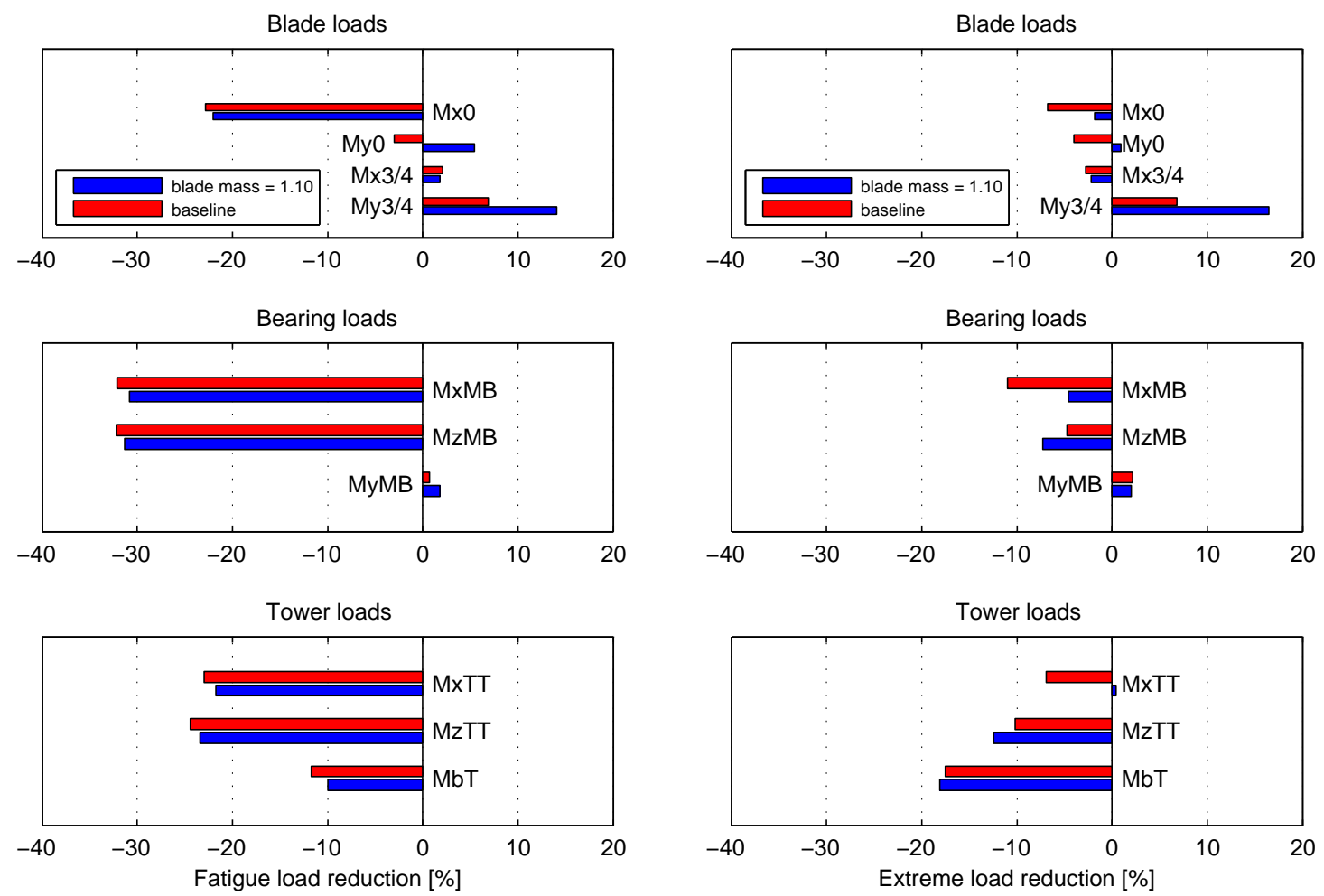

Fig. 5. Comparison of the fatigue load reduction due to the trailing edge flaps on the baseline turbine (red) and on the turbine where blades are $10 \%$ heavier (blue).

duction. The pitch controller is in charge of the power regulation. The pitch controller has not been modified for the turbine with trailing edge flaps, it is optimised for the turbine without trailing edge flaps. As a consequence, the power production of the turbine with TEF is up to $6 \%$ lower than for the baseline turbine (Figure 7), which represents a significant reduction in the annual energy production of the turbine. The price of the energy loss is not negligible, it is estimated to be of the order of magnitude of a few percent of the price of the turbine. This loss would be even higher if the energy necessary to actuate the flaps was taken into account.

However, power loss could be reduced by designing a common controller for the pitch system and the trailing edge flaps.

2) TEF activity: The tuning of the controller is a trade-off between load reduction and the wear and tear of the trailing edge flap actuators. An estimate of the wear of the actuators is the total travel distance of the flap $D$, defined

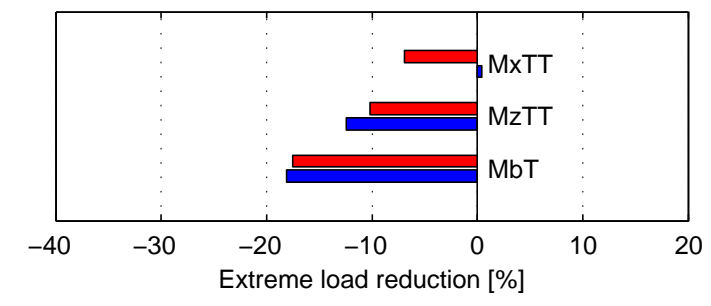

Fig. 6. Comparison of the extreme load reduction due to the trailing edge flaps on the baseline turbine (red) and on the turbine where blades are $10 \%$ heavier (blue).

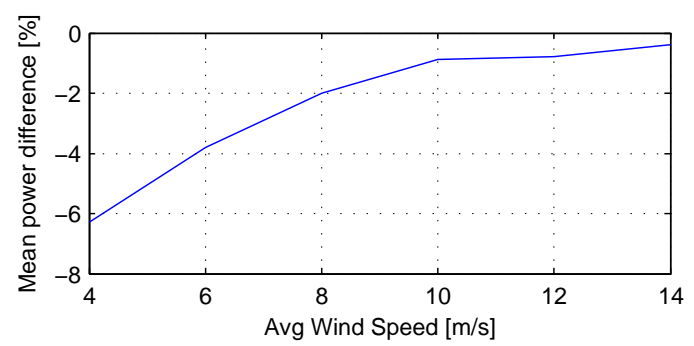

Fig. 7. Power loss as a function of the mean wind speed.

by

$$
D=\int_{0}^{20 \text { years }}\left|\frac{\partial \beta}{\partial t}\right| d t
$$

For the baseline controller, the total travel distance of the flaps is several billion degrees for a period of 20 years. Reliability of such actuators is definitely an issue.

\section{CONCLUSION}

Simulations show that trailing edge flaps are efficient to reduce most of the loads, both fatigue and extreme, in the main components 
of the turbine. Edgewise loads and driving moment loads in the main bearing are nevertheless not benefiting from the trailing edge flaps as much as the other loads, and may even be increased. Extreme loads in the normal production load cases are not reduced as much as the fatigue loads are. If extreme loads are the design criterion of some turbine components, then the benefits from having trailing edge flaps will not be as high as if only fatigue loads are taken into account. Some specific controllers to tackle extreme loads would then be necessary.

The load reduction is however achieved at a cost. With the TEF controller presented in this paper, which is independent of the pitch controller, the power production is decreased by up to $6 \%$ at low wind speeds. The energy necessary to actuate the flaps has not been taken into account, and should be added to these energy losses. Designing a common controller for both the blade pitch and the trailing edge flaps would reduce the energy loss though.

A major challenge is the design of the trailing edge flap actuators: they have to be light, to have a low power comsumption and to be reliable. The total travel distance of the flaps is in the order of magnitude of several billions degrees over 20 years.

Trailing edge flaps will be economically viable only if the turbine cost reduction due to the load alleviation is high enough to compensate for the energy loss, the price of the actuators and their maintenance. Simulations showed that high fatigue load reduction could be achieved. Controllers should now be developped in order to limit the power loss and to decrease the extreme loads.

\section{ACKNOWLEDGMENTS}

This work has been done within the frame of the Adaptive Trailing Edge Flaps (ATEF) project, partially funded by the Danish National Advanced Technology Foundation (Højteknologifonden).

\section{REFERENCES}

[1] T. Buhl, M. Gaunaa, and C. Bak, "Potential load reduction using airfoils with variable trailing edge geometry," Journal of Solar Energy Engineering, vol. 127, pp. 503-516, November 2005.

[2] M. Lackner and G. van Kuik, "A comparison of smart rotor control approaches using trailing edge flaps and individual pitch control," Wind Energy, vol. 13, pp. 117-134, 2010.
[3] P. B. Andersen, M. Gaunaa, C. Bak, and T. Buhl, "Deformable trailing edge flaps for modern megawatt wind turbine controllers using strain gauge sensors," Wind Energy, vol. 13, no. 2, pp. 193-206, 2010.

[4] T. Barlas and G. van Kuik, "Review of state of the art in smart rotor control research for wind turbines." Prog Aerospace Sci, 2009.

[5] D. G. Wilson, D. E. Berg, M. F. Barone, J. C. Berg, B. R. Resor, and D. W. Lobitz, "Active aerodynamic blade control design for load reduction on large wind turbines." Marseille, France: European Wind Energy Conference \& Exhibition, March 2009.

[6] V. A. Riziotis and S. G. Voutsinas, "Aero-elastic modelling of the active flap concept for load control." Brussels, Belgium: European Wind Energy Conference \& Exhibition, April 2008.

[7] D. Castaignet, J. J. Wedel-Heinen, T. Kim, T. Buhl, and N. K. Poulsen, "Results from the first full scale wind turbine equipped with trailing edge flaps." Chicago, Illinois: 28th AIAA Applied Aerodynamics Conference, June 2010.

[8] D. E. Berg, D. G. Wilson, B. R. Resor, J. C. Berg, T. Barlas, A. Crowther, and C. Halse, "System id modern control algorithms for active aerodynamic load control and impact on gearbox loading." Crete, Greece: TORQUE 2010: The Science of Making Torque from Wind, June 2010.

[9] "IEC 61400-1 Ed3: Wind turbines - part 1: Design requirements."

[10] M. A. Lackner and G. A. M. van Kuik, "The performance of wind turbine smart rotor control approaches during extreme loads," Journal of Solar Energy Engineering, vol. 132, 2010.

[11] S. Øye, "Dynamic stall simulated as time lag of separation." Thessaloniki, Greece: European Wind Energy Conference EWEC, October 1994.

[12] P. B. Andersen, M. Gaunaa, C. Bak, and M. H. Hansen, "A dynamic stall model for airfoils with deformable trailing edges," Wind Energy, vol. 12, no. 8, pp. 734-751, 2009.

[13] M. O. L. Hansen, Aerodynamics of Wind Turbines. Earthscan, 2008.

[14] D. Castaignet, N. K. Poulsen, T. Buhl, and J. J. Wedel-Heinen, "Model predictive control of trailing edge flaps on a wind turbine blade," American Control Conference. To be published, 2011.

[15] T. J. Larsen, H. A. Madsen, and K. Thomsen, "Active load reduction using individual pitch, based on local blade flow measurements," Wind Energy, vol. 8, no. 1, pp. $67-80,2005$.

[16] D. E. Berg, D. G. Wilson, B. R. Resor, M. F. Barone, and J. C. Berg, "Active aerodynamic blade load control impacts on utility-scale wind turbines." Chicago, IL: AWEA Windpower, 2009. 


\section{Paper D}

Robustness assessment of a flap controller with two alternative aeroelastic simulation tools

Paper submitted at the Wind Energy journal.

This work has been submitted to the Wind Energy journal for possible publication. Copyright may be transferred without notice, after which this version will be superseded. 


\title{
Robustness assessment of a flap controller with two alternative aeroelastic simulation tools
}

\author{
Damien Castaignet ${ }^{1}$, Leonardo Bergami ${ }^{2}$, Thomas Buhl ${ }^{2}$, Niels K. Poulsen ${ }^{3}$ and Jens Jakob Wedel- \\ Heinen $^{1}$. \\ ${ }^{1}$ Vestas Wind Systems A/S, Global Research \& Innovation, Roskilde, Denmark. \\ ${ }^{2}$ Risø DTU, National Laboratory for Renewable Energy, Roskilde, Denmark. \\ ${ }^{3}$ Technical University of Denmark, DTU Informatics, Lyngby, Denmark.
}

\begin{abstract}
Simulations of a multi-megawatt wind turbine with distributed trailing edge flaps are run with the aeroelastic code Flex5 and with several model complexities of the aeroelastic code HAWC2. The performance of the frequency-weighted model predictive control of trailing edge flaps run with the different simulation models is studied, below, around and above rated power. The introduction of the blade torsion degree of freedom in HAWC2 reduces the efficiency of the trailing edge flaps: an increased flap activity and a decreased flapwise blade root fatigue load reduction are observed. The multi-body modeling of the blades is important around rated power, where the blade deflections are the largest. An unstability, which does not happen with the single body formulation, is observed with the multi-body modeling of the blades. Running the controller on different simulation models makes it possible to tune the controller in order to increase the business case certainty before implementing trailing edge flaps on a real wind turbine. Copyright (c) 2010 John Wiley \& Sons, Ltd.
\end{abstract}

\section{KEYWORDS}

trailing edge flap; aeroservoelastic code; load reduction

\section{Correspondence}

D. Castaignet, Vestas Wind systems A/S, Frederiksborgvej 399, DK-4000, Roskilde, Denmark.

E-mail:daca@vestas.com

\section{Contract/grant sponsor}

This work was supported by the Danish National Advanced Technology Foundation (Højteknologifonden), as part of the ATEF (Adaptive Trailing Edge Flaps) project.

Received...

\section{INTRODUCTION}

Wind turbines rotor size has increased significantly over the last years, with most wind turbine manufacturers marketing turbines with rotor diameters over $100 \mathrm{~m}$. Increasing the blade span is an effective way to increase the power production of the turbine, but it comes at the cost of higher loads on all the turbine components. Higher fatigue and extreme loads means more material or more expensive material to design the components, increasing the capital cost of the wind turbine. Reducing the cyclic loads is one of the options to keep reducing the cost of wind energy.

Loads in a wind turbine have different sources: wind conditions, such as wind turbulence or wind shear, tower shadow, resonance of wind turbine modes, yaw misalignment of the turbine, emergency shutdowns, grid loss etc [15]. Some modern wind turbines use cyclic pitch or individual pitch in order to alleviate some of the loads. Cyclic pitch, which originates from helicopter control, consists in pitching the three blades with a 120 degrees phase shift. It is used to alleviate tilt and yaw loads on the rotor. In individual pitch control (IPC), each blade can pitch independently of the two other blades. IPC is the one of the most advanced active control to alleviate loads in today's turbines [12, 13, 27, 34, 24], but its performance is limited by the relatively slow pitch actuators and by the necessity to limit pitch actuation in order to preserve the actuators and the blade bearings. Some passive load controls, like prebent blades, swept blades [5, 22] or bending/twist-coupled blades $[33,16]$, show also promising load reductions.

In order to reduce even further and in a more efficient way the loads on the rotor, several concepts of "smart" rotors have been investigated. A "smart" rotor consists in distributed sensors (accelerometers, strain gauges, Pitot tubes, pressure tabs etc.) and actuators (trailing edge flaps, microtabs, boundary layer suction or blowing jets, plasma actuators etc.) along the blades. A detailed overview of different "smart" rotor concepts is given in [7].

Trailing edge flaps on turbine blades have been investigated for several years. CFD simulations [9] and 2D $[8,14,10]$ and 3D $[4,18]$ aeroelastic simulations confirmed the high potential of trailing edge flaps to reduce blade root 
fatigue loads. Wind tunnel tests on a blade section [6, 38, 2] as well as on a scaled turbine [37] corroborated the ability of the trailing edge flaps to reduce loads. At last, a full scale test was carried out on a Vestas V27 turbine at Risø DTU $[20,17]$.

Whereas large loads reductions were achieved with aeroservo-elastic simulations, there is still a large uncertainty on the accuracy of the simulation tools and thus on the load reduction which could be achieved on a real wind turbine. This is an issue especially for modelbased controls, where the performance of the controller is directly linked to the accuracy of the design model. Tuning a controller on a real wind turbine is not an easy task either, especially when the number of sensors and actuators increases. It is thus important to test the sensitivity and the robustness of the trailing edge flaps model-based controller (frequency-weighted model predictive control) with respect to alternative simulation models. Simulations are run with Flex5 and HAWC2, which are two aero-servoelastic simulation tools whose aerodynamics and structural modeling differ considerably.

In the first section of this paper, we briefly describe the structural and the aerodynamics models implemented in the simulation tools. In section 3, we compare the loads calculated by the different simulation models on the baseline multi-megawatt turbine, without running the trailing edge flaps controller. In the last section, we study the sensitivity and the robustness of the trailing edge flaps controller.

\section{FLEX5 AND HAWC2 MODELS}

Flex5 and HAWC2 are two aero-servo-elastic codes for simulation of wind turbines. Flex 5 is developed at the Mechanical department of the Technical University of Denmark DTU-MEK, Lyngby, Denmark; HAWC2 is developed at the Wind Energy department of Ris $\varnothing$ DTU, National Laboratory for Sustainable Energy, Roskilde, Denmark.

\subsection{Structural models}

The structural modeling of the wind turbine components in HAWC2 and in Flex5 differs: a modal model is used in Flex 5 whereas HAWC2 is based on a nonlinear multibody finite element model.

\subsubsection{Flex5 structural model}

In Flex5, a beam is parameterised by its mode shapes. Assuming a non-uniform Euler-Bernoulli beam along the $x$ axis, the deflection of the beam section $x$ at time $t$ in the $y$ and $z$ directions are:

$$
\begin{aligned}
& u^{y}(x, t)=\sum_{i=1}^{N} g_{i}(t) u_{i}^{y}(x), \\
& u^{z}(x, t)=\sum_{i=1}^{N} g_{i}(t) u_{i}^{z}(x),
\end{aligned}
$$

where $g_{i}(t)$ is the generalised coordinate associated to the $i^{\text {th }}$ beam eigenmode, $u_{i}^{y}(x)$ and $u_{i}^{z}(x)$ the $i^{\text {th }}$ eigenmode deflection of the beam section $x$ respectively in the $y$ and $z$ direction, and $N$ is the number of mode shapes taken into account in the structural model.

The generalised coordinates $g_{i}$ are derived by solving Newton's second law $M_{i} \ddot{g}_{i}+C_{i} \dot{g}_{i}+K_{i} g_{i}=F_{i}$, where $M_{i}$ is the generalised mass, $C_{i}$ the generalised damping, $K_{i}$ the generalised stiffness and $F_{i}$ the generalised force associated to the $i^{\text {th }}$ eigenmode:

$$
F_{i}(t)=\int p^{y}(x, t) u_{i}^{y}(x) \mathrm{d} x+\int p^{z}(x, t) u_{i}^{z}(x) \mathrm{d} x .
$$

$p^{y}(x, t)$ and $p^{z}(x, t)$ are the loads (aerodynamic, inertial, gravity etc.) acting on the beam section $x$, respectively in the $y$ and $z$ direction.

The tower, the drive train and the blades are modeled with this modal approach. Figure 1 shows the four first eigenmodes of a small-size blade. $u_{z}$ is the flapwise deflection, and $u_{y}$ the edgewise deflection. The first and third eigenmodes are dominated by flapwise deflections. Taking into account the first 2 to 6 mode shapes of the tower, the shaft and the blades is usualy sufficient to represent accurately the wind turbine dynamic response. The other eigenmodes have high eigenfrequencies which are hardly excited, and are thus not required in the wind turbine model. The Flex 5 structural model has the advantage of being fast to compute because the beams deflections are parameterised by only a few states. A whole wind turbine simulation runs around 10 times faster than real-time on a personal computer.

However, this modeling has known drawbacks: the absence of a blade torsion degree of freedom, and the linearity of the beam delfections. The modal modeling does not include any torsion of the beam. The torsion degree of freedom of the blades is generaly thought to be important when simulating wind turbines with trailing edge flaps because the flaps increase significantly the aerodynamic moment coefficient of the airfoil, leading to a twist of the loaded blade. The modal formulation of Flex 5 is linear, so non-linear effects, as for instance couplings of different modes due to large deflections are not taken into account.

\subsubsection{HAWC2 structural model}

The structural model in HAWC2 is based on a multi-body finite element formulation [26].

The wind turbine structure is described by a number of bodies. Following a floating frame of reference formulation [35], each body is defined in its local coordinate reference system, which can translate and rotate with respect to 

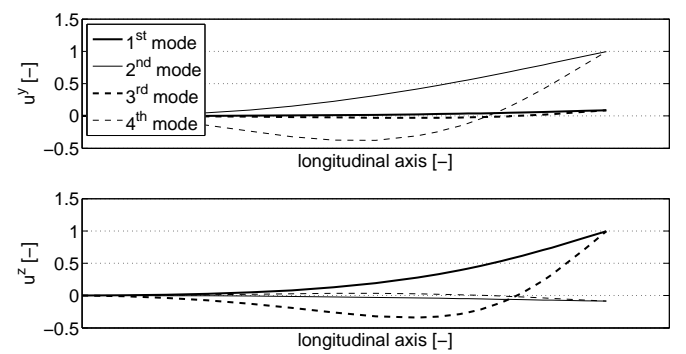

Figure 1. Four first eigenmodes of a wind turbine blade, in flapwise and edgewise directions.

the inertial reference frame. Physical constraints among the wind turbine components are modeled by algebraic equations limiting the motion of the corresponding bodies.

Structural deformations are computed inside each body, with respect to the local reference frame. Deformations are solved using a finite element approach, where the body is modeled as a sequence of Timoshenko beam elements, accounting also for beam shear and torsion effects.

Inside each body, the structural deformations are computed using a linear formulation, and small deflections and rotations are assumed. Non-linear effects are modeled by representing a structural component, e.g. a blade, as a series of bodies. The (linear) deformation state of the last element of a body is then passed to the following body by translating and rotating its local reference frame. The multibody formulation allows thus to capture non-linear effects, as, for instance, the effects of large blade deflections and coupling of torsion to blade bending.

Three different set-ups of the HAWC2 structural model are investigated, with an increasing degree of complexity of the structural model:

1. HAWC2-ST (Stiff in Torsion). The HAWC2 model is set-up in order to return a structural response that resembles the most the Flex 5 response. Each structural component is modeled as a single body, thus giving a linear formulation. The beam properties of the structure are modified to make blades and tower stiff in torsion; large Timoshenko shear coefficients are applied to make the beam elements more similar to Euler-Bernoulli elements.

2. $H A W C 2-1 B$ (1 Body). The torsion and shear properties of the beams are modeled; a linear formulation is maintained by using one body for each structural component. The torsion degree of freedom is thought to be particularly relevant in the modeling of a blade with flap, as the flap deflection introduces a significant variation in the aerodynamic pitching moment.

3. HAWC2-MB (Multiple Bodies). The wind turbine blades are now described by multiple (nine) bodies, thus accounting for non-linear effects in the structural response. Torsion and shear properties are also modeled.

\subsection{Aerodynamic models}

The aeroelastic simulation tools HAWC2 and Flex5 follow a Blade Element Momentum (BEM) method to model the aerodynamic response of the rotor.

Each of the two BEM models is organized on two interdependent levels. The first level, blade section aerodynamic, consists of a $2 \mathrm{D}$ aerodynamic model that returns the unsteady forces and the aerodynamic moment for each blade element section. On the second level, the rotor induction dynamics are determined under the BEM assumption of independent annular rotor elements, and a dynamic inflow model is used to compute the induced velocities over the rotor area.

\subsubsection{Blade section aerodynamics}

The blade section aerodynamics are based, both in HAWC2 and Flex5, on the ATEFlap aerodynamic model [11]. The model takes as input the steady aerodynamic coefficients for the airfoil section and the flap, and it returns lift, drag, and moment responses to flap deflections and changes in the section oncoming flow.

The aerodynamic response from the model includes dynamic effects of three types:

Added mass or non-circulatory effects describe the forces that arise as a reaction to the fluid accelerated by the airfoil or the flap motions; they have no memory effect and only depend on the instantaneous motion of the blade section or flap.

Shed vorticity or potential flow effects, account for the response dynamics induced by the vorticity shed in the airfoil wake following a change of the airfoil circulation.

Dynamic stall effects represent the dynamics of flow separation on the airfoil.

The potential flow part of the model is based on Gaunaa's [21] thin airfoil model, where the effects of the vorticity shed into the wake on the circulatory lift are represented through a superposition of indicial lift responses of the Wagner-type. The same indicial response function describes the circulatory response to changes either in the airfoil flow conditions, or flap deflection angle; the function is formulated in exponential terms, thus allowing for a computationally efficient integration of the superposition integral.

In the ATEFlap model, the potential flow part is coupled with the Beddoes-Leishmann dynamic stall model described by Hansen et al. [23]. The circulatory lift is computed as a weighted sum of a fully attached (potential flow) contribution, and a fully separated contribution. The weight factor in the sum accounts for the dynamics of flow separation.

A more detailed description of the ATEFlap model, and its implementation in the aeroelastic tool HAWC2 is available in Bergami and Gaunaa [11].

\subsubsection{Rotor induction}

Both aeroleastic codes apply BEM theory with Glauert and Prandtl corrections to determine the induced velocities 
over the rotor area $[36,32,25]$. The dynamics of the rotor induction are described by a dynamic inflow model, which accounts for the delay in the response of the induced velocities to changes in the rotor loading conditions; in Flex 5 and HAWC2, the dynamic inflow is modeled as a series of two first order low-pass filters [36].

Although based on the same theory, the two aeroelastic simulation tools differ in the way the induced velocity models are implemented. In Flex5 [32], the classic striptheory approach is adopted, and the induced velocities are assumed constant over a radial annular element, for every azimuthal position. On the contrary, in the HAWC2 model, the rotor area is discretized into a polar grid, and the induced velocity are computed for each point of the grid using equivalent local thrust and torque coefficients [29]; the resulting induced velocities depend on both radial and azimuthal positions.

In case of non-uniform wind field, as for instance due to wind shear, the two aeroelastic codes return slightly different aerodynamic responses.

A limitation of the described rotor aerodynamic models, both in HAWC2 and Flex5, lies in the assumption of the BEM theory that each annular element on the rotor area is independent from the others, and, therefore, the flow conditions at one radial position have no effect on adjacent radial elements. The assumption is violated whenever strong variations of aerodynamic loading occurs along the blade span, and such is the case at the extremities of blade sections with flaps. Abrupt loading variation in the radial direction generates trailed vorticity, which directly affects the induced velocities on the blade. The effects of trailed vorticity are not captured by the BEM method, and represents a limitation of the employed aeroelastic simulation tools; a vortex wake model would be required to overcome this limitation $[28,3]$.

\subsection{Wind}

In both Flex 5 and HAWC2, the wind field is a combination of a wind turbulence box, turbulence intensity, wind shear and wind direction. The wind turbulence boxes, based on the Mann's model [30], are generated by a program external to Flex 5 and HAWC2. The same 600 seconds turbulence boxes are used in Flex 5 and HAWC2 simulations.

Simulations are run at three different wind speeds: below rated power, at rated power and above rated power. At each wind speed, twelve different turbulent boxes are generated and simulations are run with two different wind directions as recommended in the IEC standards [1] for normal production load cases. HAWC2 and Flex5 simulations are then run with exactly the same turbulent boxes and wind conditions.

\subsection{Controllers}

Controllers are not part of the aeroelastic simulation codes but are implemented in Dynamic-Link Libraries (DLL) which are interfaced with the simulation codes Flex5 and

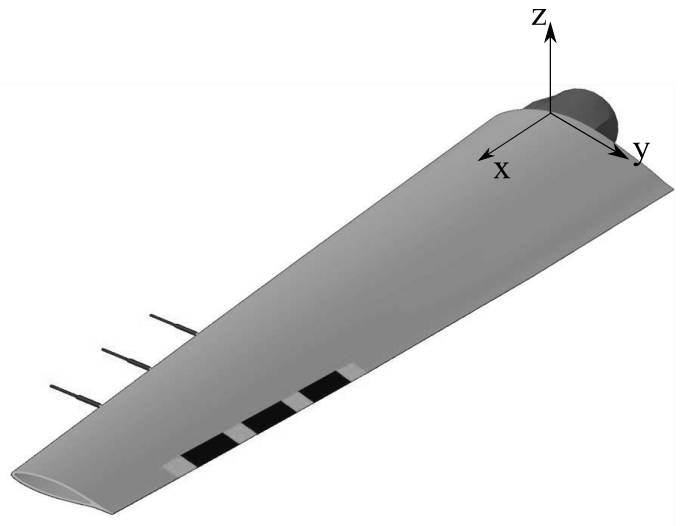

Figure 2. Illustration of a blade equipped with trailing edge flaps, inactive transition pieces and Pitot tubes.

HAWC2. The same controllers can then be sued with both aeroelastic codes.

\subsection{Fatigue loads}

The equivalent fatigue damage for the turbine components is determined using a rainflow counting algorithm, and Miner's damage accumulation rule [31]. The fatigue damage of a material going throught $N_{i}$ cycles of stress range $\sigma_{i}$ is assumed equivalent to the fatigue damage of the same material going throught $N^{e q}$ cycles with a stress range $\sigma^{e q}$, where

$$
\sigma^{e q}=\sum_{i} \sigma_{i}\left(\frac{N_{i}}{N^{e q}}\right)^{1 / m} .
$$

An approximation of the Wöhler curve is used to calculate $\sigma^{e q}$. In this paper, $m$, which is a constant specific to the material, is assumed to be 12 for the blades, 3 for the tower and 8 for the main bearing.

\section{TEST TURBINE}

\subsection{Wind turbine description}

The modeled wind turbine is a conventional multi megawatt, 3 blade, upwind, variable speed, pitch regulated turbine. The three blades are equipped with trailing edge flaps located towards the blade tip, covering $25 \%$ of the blade span. Figure 2 represents an illustration of a blade equipped with three trailing edge flaps and tested within the ATEF project [20].

Different structural and aerodynamics inputs are required in HAWC2 and in Flex5, which leads to small differences between the wind turbine models. On the other hand, the generator, the pitch and the trailing edge flap controllers are compiled into dynamic-link libraries (DLL), which ensures that the same controllers are used in Flex 5 and in HAWC2. 
They are run at the same sampling frequency, and they use sensors located at the same positions.

A wind turbine model is accurate and fits field measurements only after the wind turbine model has been tuned based on some field and test measurements. This tuning depends on the simulation tool used, and different tunings should thus be made for each of the Flex 5 or HAWC2-based simulation models. This tuning is a long and difficult task which is out of the scope of this paper. The purpose of this paper is however not to compare the different simulation models to real data, but to study the influence of those discrepancies in eigenfrequencies or in steadystate or dynamic loads on the trailing edge flap controller performance. Some baseline tests are run in order to assess those differences.

\subsection{Trailing edge flaps controller}

The objective of the trailing edge flaps controller is to reduce the flapwise blade root fatigue loads. Loads which account the most for the flapwise blade root fatigue have frequencies between the $1 \mathrm{P}$ and the $3 \mathrm{P}$ frequencies, where the $1 \mathrm{P}$ frequency corresponds to one event per rotor revolution, and $3 \mathrm{P}$ to 3 events per revolution. A frequencyweighted model predictive control [19] is designed to focus on those specific loads. The cost of this model predictive control is on the zero-phase bandpass filtered flapwise blade root moment and on the zero-phase bandstopped filtered trailing edge flaps angles. The bandwidth of the bandpass filter on the flapwise blade root moments includes the frequencies of the targeted loads, ie frequencies between the $1 \mathrm{P}$ and the $3 \mathrm{P}$ frequencies. The bandstop filter on the controller inputs is designed in order to prevent the actuation of the trailing edge flaps at frequencies lower than the $1 \mathrm{P}$ frequency and above the 3P frequency, in order to avoid inefficient high frequencies actuation of the trailing edge flaps and excitation of some unwanted wind turbine eigenmodes.

This trailing edge flap controller was originally developed in order to be tested on a Vestas V27 turbine which is equipped with three trailing edge flaps on one of its blades [20, 17]. In order to match the experimental setup, the trailing edge flap controller is made independent of the pitch controller, and the sensors available to the controller are limited to the flapwise blade root moment, the pitch position, and the local wind speeds measured by some Pitot tubes on the leading edge of the blades. Three independent identical model predictive controls run in parrallel, one for each blade.

The design model is an analytical linear model of a single blade, where the blade deflection is parameterised by its mode shapes in a way similar to Flex5. Only the first blade eigenmode is taken into account. The dynamics of the induced velocities, which are significantly slower than the lowest frequency of interest for the trailing edge flap controller (the $1 \mathrm{P}$ frequency) are neglected. The aerodynamics of the trailing edge flaps are also neglected, because they are significantly faster than the highest frequency of interest for the controller: the 3P frequency. The steady-state lift and drag coefficients of an airfoil with a trailing edge flap are considered in the design model. Those simplifications lead to a simple linear model with only two states: the generalised coordinate $g_{1}$ associated to the first blade eigenmode, and its derivative $\dot{g}_{1}$. Figure 3 shows the Bode plots, from trailing edge flap angle to flapwise blade root moment of the Vestas V27 turbine. The Bode plots are simulated with Flex5, with maximum trailing edge flap stroke, with two wind turbine models: a turbine modeled as a stiff turbine except for the first blade eigenmode (Flex - Stiff turbine) and a turbine with flexible tower, shaft and blades (Flex - Flexible turbine). The Bode plot of the analytical linear blade model is very similar to the Bode plots simulated with the flexible turbine model in Flex5. The small differences around $1 \mathrm{P}$ and $5 \mathrm{P}$, in this specific example, are due to some eigenmodes involving the tower fore-aft deflection and the blade second eigenmode which are not modeled in the design model.

The linear blade model is agregated with some disturbance states which model the $1 \mathrm{P}, 2 \mathrm{P}$ and $3 \mathrm{P}$ content of the wind speed seen by the spinning blade. The design model states are estimated by a predictive Kalman filter.

The controller and the Kalman filter are fine-tuned by running Flex5 simulations only, until a good performance of the controller is reached. No further tuning is made with HAWC2 simulations.

\section{BASELINE TESTS}

The baseline tests are run with the generic multi-megawatt turbine described in Section 3. Efforts were made to have similar wind turbines models in Flex 5 and HAWC2, but there are still significant differences between the results of the different simulations models, partly due to differences in the structural and aerodynamic inputs to the model, and partly due to the difference in the simulation models (structural and aerodynamic modeling). Some baseline tests are carried out by simulating the aeroelastic response of the wind turbine models with the Flex5 and HAWC2 codes. The differences in the simulated responses are quantified, and their main sources are located. In this way, we obtain a term of comparison for the following active load reduction cases; the comparison will be useful in identifying which differences in the simulated responses are related to the flap control system or to the aeroelastic modeling.

\subsection{Wind turbine eigenfrequencies}

First, the eigenfrequencies of the wind turbine in standstill, without any aerodynamic or centrifugal loads, are compared. They indicate differences between the underlying structural models. Eigenfrequencies in HAWC2 can only be calculated for the single body models.

The most important eigenfrequencies are summarised in Table I, where the difference with respect to the eigenfrequencies as calculated by Flex 5 are given. 

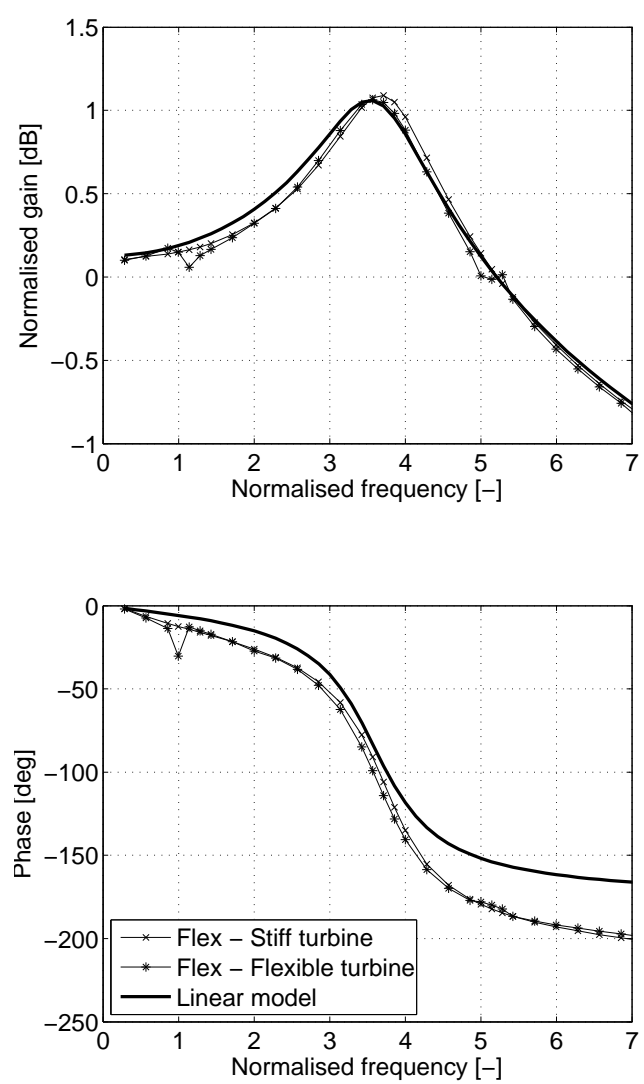

Figure 3. Bode plot for the SISO system trailing edge flap angle to flapwise blade root moment. The $x$ markers refer to simulations run in Flex, with a stiff turbine where only the first blade eigenmode is flexible. The star markers refer to simulations run in Flex with the model of a turbine where all the main turbine components are modeled as flexible structures. The thick line refers to the analytical linear model as described in this paper.

The frequencies are normalised with the 1P frequency.

Eigenfrequencies of the HAWC2-ST model are relatively close to the eigenfrequencies of the Flex 5 model, and are within the range of eigenfrequency drift that can be expected on real turbines.

The modeling of the blade shear and torsion increases significantly the frequency of the flapwise-yaw eigenmode.

\subsection{Steady-state conditions}

Comparing the steady-state loads gives indication on the steady behavior of the aerodynamic modeling. All four models are run with a constant uniform wind, a constant rotor speed and a constant pitch angle in order to check the quasi-steady mean flapwise blade root moment and aerodynamic power.
Table I. Wind turbine eigenfrequencies comparison. Eigenfrequencies are expressed in percentage of difference with the eigenfrequencies of the Flex5 model. Only the most important eigenfrequencies are given.

\begin{tabular}{ccc} 
& HAWC2-ST & HAWC2-1B \\
\hline \hline Tower side-side & $+0.6 \%$ & $+0.9 \%$ \\
Tower fore-aft & $-0.3 \%$ & $-0.0 \%$ \\
Flapwise - Yaw & $+2.9 \%$ & $+11.2 \%$ \\
Flapwise - Tilt & $+3.9 \%$ & $+4.1 \%$ \\
Flapwise - collective & $+6.1 \%$ & $+6.3 \%$ \\
Asym. Edge - Up Down & $-9.3 \%$ & $-8.6 \%$ \\
Asym. Edge - Side & $+0.3 \%$ & $+1.0 \%$ \\
\hline
\end{tabular}

The quasi-steady flapwise blade root moment of the HAWC 2 models differ by less than $8 \%$ to the Flex 5 result, and the aerodynamic power, below rated power, by less than $4 \%$.

\subsection{Turbulent wind field, trailing edge flaps fixed}

This baseline test consists in running Flex5 and HAWC2 simulations, with the trailing edge flaps fixed in their neutral position, below rated power, around rated power and above rated power. The purpose of this test is to compare the servoaero-elastic models without the influence of the trailing edge flaps dynamics and the trailing edge flaps controller. The simulations are run with the twelve different wind turbulence boxes and for two wind directions.

The averaged spectral densities of the flapwise blade root moment do not show any major difference between the simulation models, except for a peak around 7.5P, below rated power, with the $H A W C 2-M B$ simulation model only (Figure 4). The 7.5P frequency corresponds to an eigenmode of the turbine which involves mainly the blade edgewise eigenmode. The multibody modeling of the blades accounts for the coupling between flapwise and edgewise deformation modes caused by the blade deflection. These non-linear coupling effects lead to a higher excitation of the blade edgewise mode, at frequencies close to $7.5 \mathrm{P}$, in this example.

The largest differences in flapwise blade root fatigue loads, between the Flex5 and the HAWC2 simulation models, are reported around rated power (Table II), when the blade deflection is the highest and thus the structural modeling of the blade has a high influence on the flapwise blade root moment.

Above rated power, the angles of attack at the blade sections are lower, attached flow condition prevails, and the blade deflection is lower: the differences in fatigue flapwise blade root loads between the simulation models are no longer significant (below 4.3\%).

Fatigue loads in the tower and the main bearing also differ significantly from a simulation model to another (Table II). Results from the HAWC2-ST simulation model, which was created to resemble the Flex 5 model as close as possible, are not closer to the Flex 5 results than the other HAWC2 simulation models. 

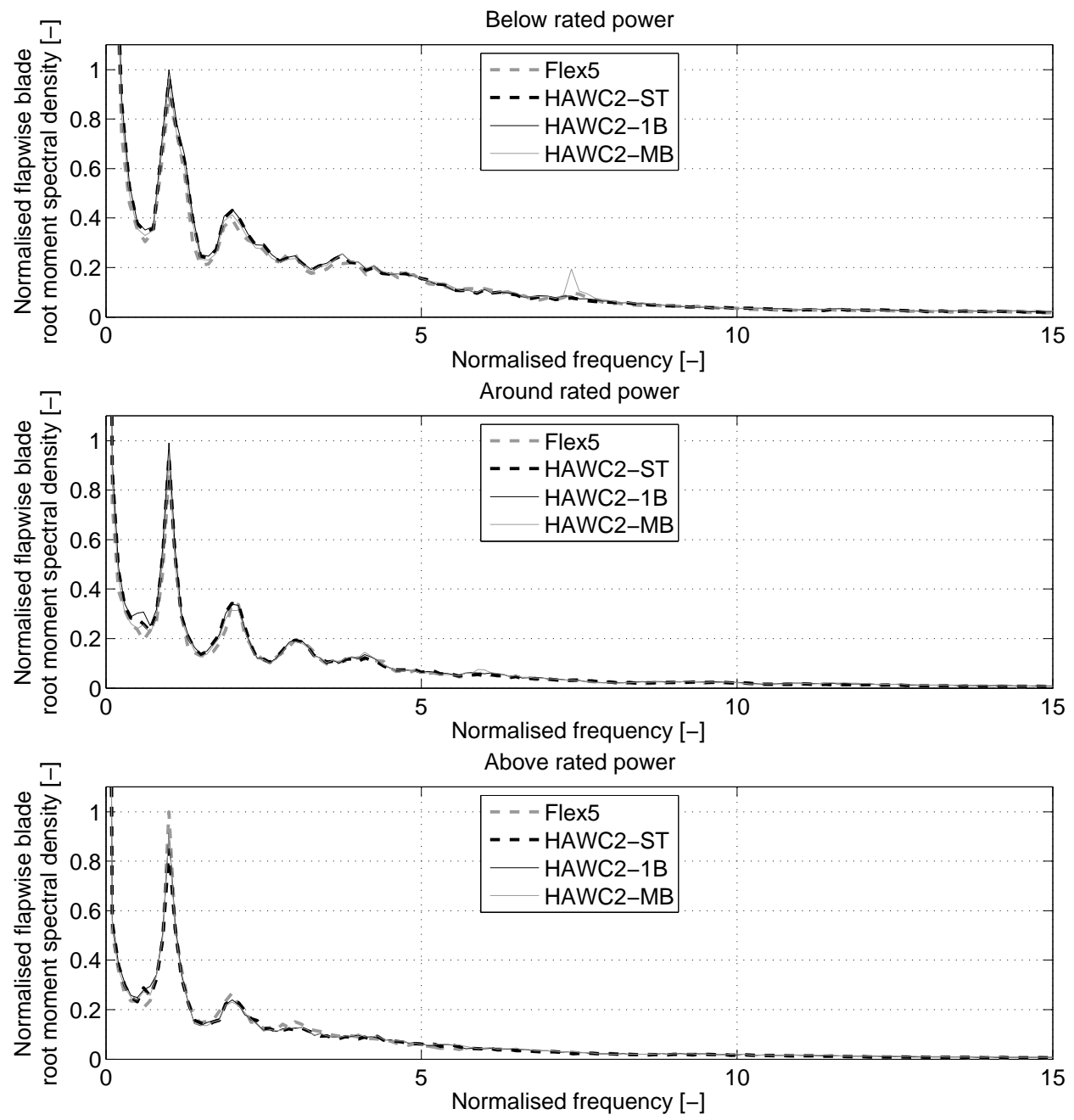

Figure 4. Spectral densities of the flapwise blade root moment, below rated power (top), around rated power (middle) and above rated power (bottom). Results are showed for the following simulation models: Flex5 (thick dashed grey), HAWC2-ST (thick dashed black), $H A W C 2-1 B$ (thin black) and HAWC2-MB (thin grey). Frequencies are normalised with the 1P frequency.

\subsection{Trailing edge flap step function}

The Flex5 and HAWC2 simulation models are run at a constant wind speed below rated power, at a constant rotor speed and a constant pitch angle. A step function is applied to the trailing edge flaps at time $t=0$, from their neutral position to their maximum lift position (Figure 5).

In the first instants following the step in trailing edge flap deflection, the lift coefficients computed by Flex5 and by HAWC2-ST match, thus confirming that the $2 \mathrm{D}$ blade section aerodynamics and the related inputs are the same in both simulation codes. As the time passes, the two lift coefficient curves drift slightly away, mainly as an effect of the differences in the induced velocities modeling. Notice that the differences right after the trailing edge flap step are due to a larger time step used in Flex 5 than in HAWC2 when solving the differential equations.
The differences in angle of attack between the simulation models are larger, which confirms the difference in the structural responses predicted by Flex 5 and HAWC 2 .

Comparing the results from the HAWC2-ST and the $H A W C 2-1 B$ simulation models shows the importance of the torsion degree of freedom in the blade model, when trailing edge flaps are used. At a given blade section, a trailing egde flap in its maximum lift position increases significantly the local moment coefficient, leading to a twist of the blade and a relative decrease of the local angles of attack. In steady conditions, in this example, this leads to a lower lift coefficient, and a $6 \%$ loss in flapwise blade root moment.

The flapwise blade root moment reflects the differences already seen in the time series of the angle of attack and lift coefficient. The four simulation models show the same behavior of the flapwise blade root moment right after the trailing edge flap step: the flapwise moment first decreases 

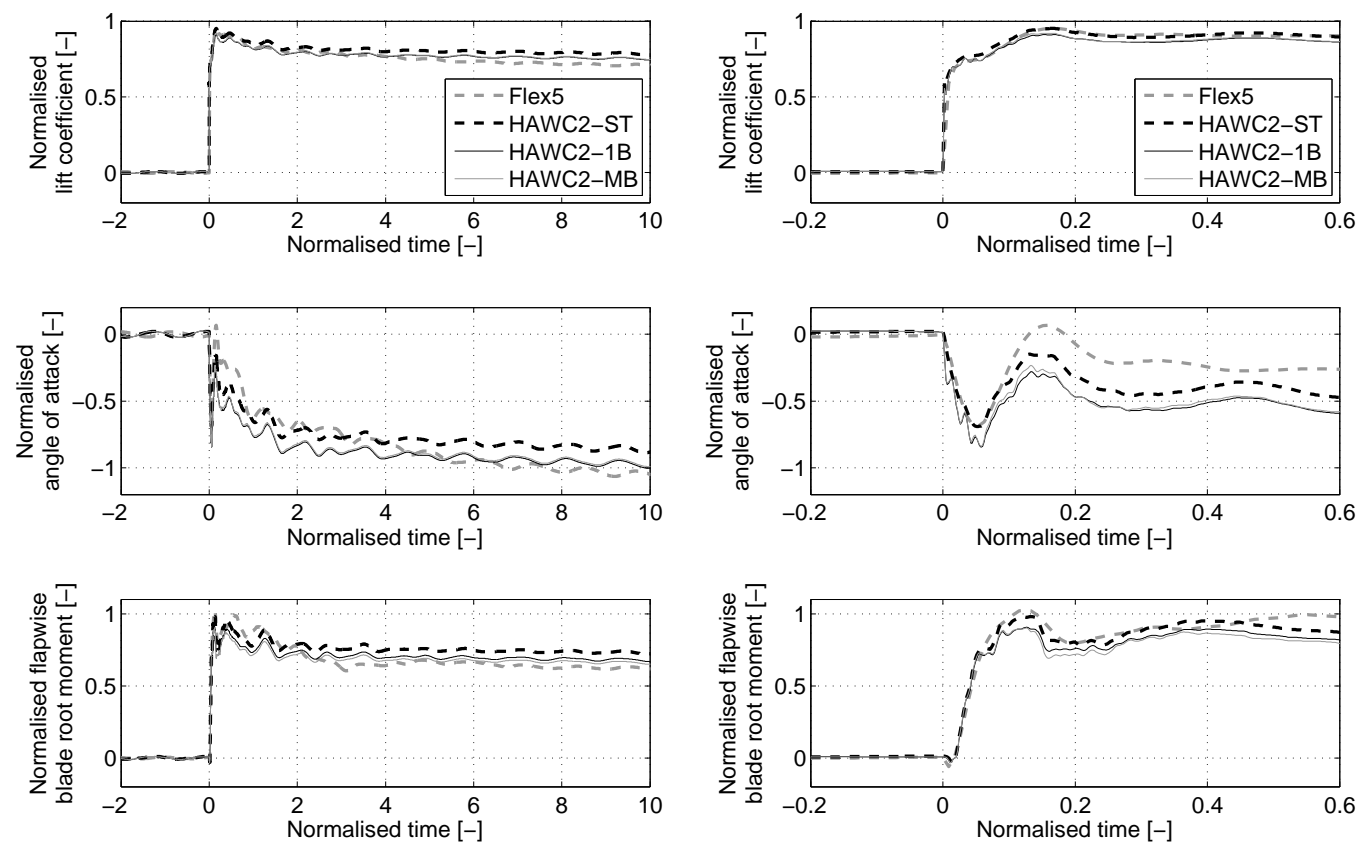

Figure 5. Local lift coefficient (top) and local angle of attack (middle) of a blade section, and flapwise blade root moment (bottom) when the trailing edge flap angle is increased at time $t=0$, according to a step function. Time is normalised to the time the wind needs to travel one rotor diameter. The right hand side plot is a zoom around $t=0$ of the left hand side plot.

for a very short time, and then increases (non minimum phase zero).

Those baseline tests showed that the aeroleastic codes return slightly different responses. None of those simulation models, as complex as they are, calculate the structural and aerodynamic responses that is observed on a real wind turbine. However, those differences are large enough to be able to test the sensitivity and the robustness of the trailing edge flap controller, but small enough to consider that they model the same wind turbine. Running simulations with all those simulation models gives an indication of the differences that can be expected by testing trailing edge flaps on a real wind turbine.

\section{SENSITIVITY OF THE TRAILING EDGE FLAPS CONTROLLER TO THE SIMULATION MODELS}

\subsection{Simulation setup}

Simulations of the generic multi-megawatt trubine described in Section 3 are run, for each simulation model (Flex5, HAWC2-ST, HAWC2-1B, HAWC2-MB) with and without the trailing edge flap controller. Fatigue load reductions are always calculated as the difference in fatigue loads between the simulations with the trailing edge flaps active and with the trailing edge flaps fixed in their neutral positions, with the same simulation model. Results of the 12 simulations at each wind speed are averaged.

The trailing edge flap activity is estimated by calculating the trailing edge flap travel distance $d$, defined as

$$
d=\int\left|\frac{\partial \beta}{\partial t}\right| \mathrm{d} t
$$

where $\beta$ is the trailing edge flap angle.

\subsection{Results}

Results of flapwise blade root fatigue load reduction and trailing edge flap travel distance are summarised in Table III. The flapwise blade root fatigue load reduction achieved with the frequency-weighted model predictive control of the trailing edge flaps differ a few percent depending on the simulation model used. The differences are however small compared to the difference in equivalent fatigue loads seen when the trailing edge flaps are fixed in their neutral position (Table II). The HAWC2-ST simulations, which have returned higher fatigue loads than the Flex5 simulations when the trailing edge flaps were fixed, also return higher active load alleviation. The same results are observed for the main bearing loads and the tower bottom loads. 
Table II. Difference in fatigue loads between the Flex5 simulation model and respectively, from top to bottom, the HAWC2-ST, HAWC2-1B and HAWC2-MB simulation models. Fatigue loads are calculated for the flapwise blade root moment (Flapw. BRM), the yaw and tilt moment in the main bearing (Br. Yaw and $\mathrm{Br}$. Tilt) and the tower bottom (Tower), below, around and above rated power.

\begin{tabular}{cccc} 
\% HAWC2-ST & below & around & above \\
\hline \hline Flapw. BRM & $+7.4 \%$ & $+15.0 \%$ & $+1.5 \%$ \\
Br. Yaw & $-6.6 \%$ & $-6.3 \%$ & $-13.9 \%$ \\
Br. Tilt & $+4.4 \%$ & $+10.9 \%$ & $+7.1 \%$ \\
Tower & $+0.1 \%$ & $-4.1 \%$ & $+1.1 \%$ \\
\hline \multicolumn{4}{c}{} \\
\% HAWC2-1B & below & around & above \\
\hline \hline Flapw. BRM & $+10.0 \%$ & $+18.0 \%$ & $+4.3 \%$ \\
Br. Yaw & $-5.7 \%$ & $-5.7 \%$ & $-11.2 \%$ \\
Br. Tilt & $+8.2 \%$ & $+15.6 \%$ & $+11.1 \%$ \\
Tower & $+3.1 \%$ & $+4.2 \%$ & $+8.5 \%$ \\
\hline \multicolumn{4}{c}{} \\
\% HWC2-MB & below & around & above \\
\hline \hline Flapw. BRM & $+4.3 \%$ & $+9.0 \%$ & $+1.2 \%$ \\
Br. Yaw & $-3.1 \%$ & $-8.3 \%$ & $-8.8 \%$ \\
Br. Tilt & $+14.1 \%$ & $+14.3 \%$ & $+13.9 \%$ \\
Tower & $+7.2 \%$ & $+1.5 \%$ & $+10.8 \%$ \\
\hline
\end{tabular}

The trailing edge flap travel distance varies significantly from a simulation model to another: the more the simulation model differs from the Flex5 model, the higher the trailing edge flap travel distance is. This extra trailing edge flap travel distance could be partly the consequence of the unappropriate tuning of the controller for the specific simulation model and partly inherent to the simulation models (torsion degree of freedom, multi-body modeling of the blades). The comparison of the fatigue load reduction and the trailing edge flap travel distance achieved with the $H A W C 2-S T$ and the $H A W C 2-1 B$ simulation models shows that, at each wind speed, a lower flapwise blade root fatigue load reduction is achieved at the cost of a higher trailing edge flap travel distance when the torsion of the blade is taken into account. This is in agreement with the results of the trailing edge flap step simulations: taking into account the torsion degree of freedom of the blade reduces the efficiency of the trailing edge flap on the flapwise blade root moment.

The non-linear effects that are introduced in the model by using a multibody formulation (from $H A W C 2-1 B$ to $H A W C 2-M B)$ have a small effect on the load reduction capabilities below and above rated power; a decreased load reduction is however observed around rated power, where the non-linear effects due to large blade deflections are more significant. Introducing the multibody formulation yields to a significant increase of the flap deflection activity $(+125 \%)$. The spectral densities of the flapwise blade root moment and the trailing edge flap angle show an unstability at a frequency around 12P (Figure 6). When the turbine reaches rated power, the pitch controller and the trailing edge flap
Table III. Flapwise blade root fatigue loads reduction (Flapw. BRM) and trailing edge flap travel distance $(d)$ calculated for the fours simulation models. The trailing edge flap travel distance is expressed in percentage w.r.t. the trailing edge flap travel distance calculated for the Flex5 simulations.

\begin{tabular}{cccc} 
Flapw. BRM & below & around & above \\
\hline \hline Flex5 & $-14.4 \%$ & $-26.9 \%$ & $-22.7 \%$ \\
$H A W C 2-S T$ & $-16.3 \%$ & $-32.7 \%$ & $-21.6 \%$ \\
$H A W C 2-1 B$ & $-15.1 \%$ & $-28.1 \%$ & $-20.4 \%$ \\
$H A W C 2-M B$ & $-15.1 \%$ & $-24.4 \%$ & $-20.9 \%$ \\
\hline \multicolumn{4}{c}{} \\
\hline below & around & above \\
\hline \hline$H A W C 2-S T$ & $+5.2 \%$ & $+12.0 \%$ & $+5.3 \%$ \\
$H A W C 2-1 B$ & $+9.3 \%$ & $+18.2 \%$ & $+10.4 \%$ \\
$H A W C 2-M B$ & $+15.5 \%$ & $+124.9 \%$ & $+16.2 \%$
\end{tabular}

controller work against each other, and generate periodic actuation of both the pitch actuator and the trailing edge flaps at this frequency. Such a problem would not raise if a single controller was used for both the pitch actuator and the trailing edge flaps. However, by increasing the cost on the filtered inputs in the trailing edge flap model predictive control, results similar to the other simulation models are achieved: $25.1 \%$ flapwise fatigue load reduction achieved with $8 \%$ trailing edge flap travel distance lower than for the flex 5 simulations.

Except for this unstability observed with the HAWC2$M B$ simulation model, around rated power, no major issue with the trailing edge flap controller could be observed. Despite some rather large differences in the wind turbine eigenfrequencies and in the simulation models (torsion degree of freedom, multi-body), the trailing edge flap controller performs well at the frequencies of interest. It is however noticed that the trailing edge flaps travel distance increases significantly when simulations are run with a simulation model more complex than the Flex 5 model.

\section{CONCLUSION}

In this paper, a multi-megawatt wind turbine with trailing edge flaps was simulated in Flex5 and in HAWC2, with different model complexities. Some baseline tests, without active trailing edge flaps, highlighted the differences between the simulation models. The introduction of the torsion degree of freedom in the modeling of the blades reduces the trailing edge flap efficiency to change the flapwise blade root moment. The multi-body modeling of the blades increase the coupling between their flapwise and their edgewise modes due large blade deflections.

The trailing edge flap model predictive control, which was not explicitely designed to be robust, performs well with all the simulation models. The flapwise blade root fatigue load reduction differ only a few percent from each other. However, it is noticed that the trailing edge flap activity increases significantly as the simulation model gets 

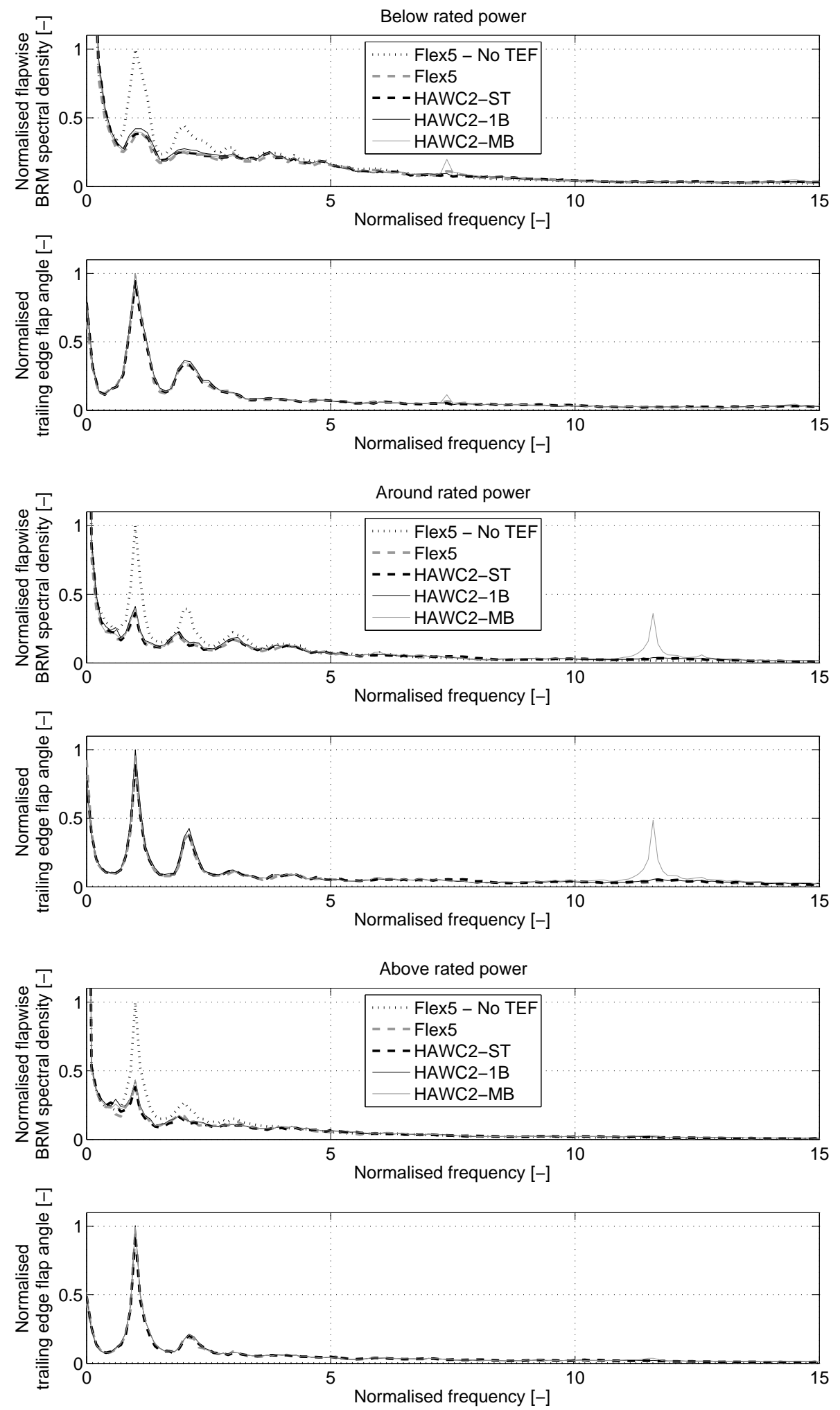

Figure 6. Spectral densities of the flapwise blade root moment and the trailing edge flap angle, below rated power (top), around rated power (middle) and above rated power (bottom). Results with active trailing edge flaps are showed for the following simulation models: Flex5 (thick dashed grey), HAWC2-ST (thick dashed black), HAWC2-1B (thin black) and HAWC2-MB (thin grey). Results of the Flex5 simulations with fixed trailing edge flaps are also plotted for comparison with the active flaps simulations (dotted line). Frequencies are normalised with the $1 \mathrm{P}$ frequency. 
more complex than the design model. This is an important issue for the wind turbine manufacturer when it comes to the design of the actuator.

The multi-body formulation of the blade model emphasises an unstability due to the pitch controller and the trailing edge flap controller working against each other. Such an unstability could not be seen with any of the other simulation models. Running the controller with different simulation models is a way to reduce the number of potential problems before going on the field, and to increase the business case certainty for the wind turbine manufacturer.

This paper only dealt with sensitivity and robustness of the trailing edge flap controller w.r.t. some of the structural and aerodynamics simulation models. Other models could also be tested, regarding for example the wake model (nearwake model [3]) or the wind model (extreme wind shear, extreme wind direction change etc.).

\section{REFERENCES}

1. IEC 61400-1 Ed3: Wind turbines - part 1: Design requirements. ISBN: 978-2-88912-201-1.

2. P. B. Andersen, C. Bak, M. Gaunaa, and T. Buhl. Wind tunnel test of a closed loop controller for an airfoil with trailing edge flaps. In TORQUE 2010: The Science of Making Torque from Wind, Crete, Greece, June 2010.

3. P. B. Andersen, M. Gaunaa, F. Zahle, and H. A. Madsen. A near wake model with far wake effects implemented in a multi body aero-servo-elastic code. In EWEC 2010, Marseille, France, 2010.

4. Peter Bjørn Andersen, Lars Henriksen, Mac Gaunaa, Christian Bak, and Thomas Buhl. Deformable trailing edge flaps for modern megawatt wind turbine controllers using strain gauge sensors. Wind Energy, 13(2-3):193-206, 2010.

5. T. D. Ashwill, G. Kanaby, K. Jackson, and M. Zutech. Development of the swept twist adaptive rotor (star) blade. In Proceedings of the 48th AIAA Aerospace Sciences Meeting Including the New Horizons Forum and Aerospace Exposition, Orlando, Florida, 2010.

6. Christian Bak, Mac Gaunaa, Peter B. Andersen, Thomas Buhl, Per Hansen, and Kasper Clemmensen. Wind tunnel test on airfoil Ris $\emptyset$-B1-18 with an active trailing edge flap. Wind Energy, 13(2-3):207-219, 2010.

7. TK Barlas and GAM van Kuik. Review of state of the art in smart rotor control research for wind turbines. Progress in Aerospace Sciences, 46(1):1-27, 2010.

8. S. Basualdo. Load alleviation on wind turbine blades using variable airfoil geometry. Wind Engineering, 29(2):169-182, Mar. 2005.

9. T. Behrens and W. Jun Zhu. Feasibility of aerodynamic flap hinge moment measurements as input for load alleviation control. In EWEA 2011, Brussels, Belgium, 2011.
10. L. Bergami and M. Gaunaa. Stability investigation of an airfoil section with active flap control. Wind Energy, 13:151-166, 2010.

11. L. Bergami and M. Gaunaa. ATEFlap aerodynamic model, a dynamic stall model including the effects of trailing edge flap deflection. Technical Report RisoeR-1792(EN), Risoe National Laboratory. Technical University of Denmark, Roskilde, Denmark, November 2011.

12. E. A. Bossanyi. Individual blade pitch control for load reduction. Wind Energy, 6(2):119-128, 2003.

13. E. A. Bossanyi. Further load reductions with individual pitch control. Wind Energy, 8(4):481-485, 2005.

14. T. Buhl, M. Gaunaa, and C. Bak. Potential load reduction using airfoils with variable trailing edge geometry. Journal of Solar Energy Engineering, 127(4):503-516, November 2005.

15. T. Burton, D. Sharpe, N. Jenkins, and E. A. Bossanyi. Wind Energy handbook. Wiley, 2008.

16. M. Capellaro and M. Kühn. Boundaries of bend twist coupling. In TORQUE 2010: The Science of Making Torque from Wind, 2010.

17. D. Castaignet, T. Barlas, T. Buhl, N. K. Poulsen, J. J. Wedel-Heinen, N. A. Olesen, C. Bak, and T. Kim. Full-scale test of trailing edge flaps on a vestas v27 wind turbine. active load reduction and system identification. [submitted].

18. D. Castaignet, T. Buhl, N. K. Poulsen, and J. J. WedelHeinen. Trailing edge flaps impact on fatigue and extreme loads in power production. In EWEA 2011, Brussels, Belgium, 2011.

19. D. Castaignet, I. Couchman, N. K. Poulsen, T. Buhl, and J. J. Wedel-Heinen. Frequency-weighted model predictive control of trailing edge flaps on a wind turbine blade. [submitted].

20. D. Castaignet, J. J. Wedel-Heinen, T. Kim, T. Buhl, and N. K. Poulsen. Results from the first full scale wind turbine equipped with trailing edge flaps. In 28th AIAA Applied Aerodynamics Conference, Chicago, Illinois, June 2010.

21. M. Gaunaa. Unsteady two-dimensional potential-fl ow model for thin variable geometry airfoils. Wind Energy, 13:167-192, 2010.

22. M. H. Hansen. Aeroelastic modal analysis of backward swept blades using HAWCStab2. Technical Report Ris $\varnothing-R-1769$ (EN), Ris $\varnothing$ DTU, National Laboratory for Sustainable Energy, February 2011.

23. M. H. Hansen, M. Gaunaa, and H. A. Madsen. A Beddoes-Leishman type dynamic stall model in statespace and indicial formulations. Technical Report R1354(EN), Risø National Laboratory, Roskilde (DK), 2004.

24. M. H. Hansen, A. Hansen, T. J. Larsen, S. Øye, P. Sørensen, and P. Fuglsang. Control design for a pitch-regulated, variable speed wind turbine. Technical Report Ris $\varnothing-R-1500(E N)$, Ris $\varnothing$ National Laboratory, January 2005. 
25. M. O. L. Hansen. Aerodynamics of Wind Turbines. Earthscan, 2008. ISBN: 978-1-84407-438-9.

26. T. J. Larsen. How 2 hawc2, the user's manual. Technical report, Ris $\varnothing-\mathrm{R}-1597$, Ris $\varnothing$, Denmark, May 2009.

27. Torben Juul Larsen, Helge A. Madsen, and Kenneth Thomsen. Active load reduction using individual pitch, based on local blade flow measurements. Wind Energy, 8(1):67-80, 2005.

28. J. Gordon Leishman. Challenges in modelling the unsteady aerodynamics of wind turbines. Wind Energy, 5(2-3):85-132, April 2002.

29. H. A. Madsen, V. Riziotis, F. Zahle, M. O.L Hansen, H. Snel, F. Grasso, T. J Larsen, E. Politis, and F. Rasmussen. Blade element momentum modeling of inflow with shear in comparison with advanced model results. Wind Energy, 2011.

30. J. Mann. Wind field simulation. Probabilistic Engineering Mechanics, 13(4):269-282, 1998.

31. J. F. Manwell, J.G. McGowan, and A. L. Rogers. Wind energy explained. Theory, Design and Application. John Wiley \& Sons Ltd, 2008. ISBN: 13:978-0-47149972-5.

32. S. Øye. FLEX4 simulation of wind turbine dynamics. In Proc. 28th Meeting of Experts, International Energy Agency, Annex XI, page 7177, 1996.

33. J. A. Paquette and P. S. Veers. Increased rotor size through passive load control and weight reduction concepts. In EWEC 2009, Marseille, France, 2009.

34. K. Selvam, S. Kanev, J. W. van Wingerden, T. van Engelen, and M. Verhaegen. Feedback-feedforward individual pitch control for wind turbine load reduction. International Journal of Robust and Nonlinear Control, 19(1):72-91, 2009.

35. A.A. Shabana. Flexible multibody dynamics: review of past and recent developments. Multibody system dynamics, 1(2):189222, 1997.

36. H. Snel and J. G. Schepers. Joint investigation of dynamic inflow effects and implementation of an engineering method. Technical Report ECN-C-94107, Netherlands Energy Research Foundation, ECN, The Netherlands, 1995.

37. J.-W. van Wingerden, A. Hulskamp, T. Barlas, I. Houtzager, H. Bersee, G. van Kuik, and M. Verhaegen. Two-degree-of-freedom active vibration control of a prototyped smart rotor. Control Systems Technology, IEEE Transactions on, 19(2):284 -296, march 2011.

38. J. W. van Wingerden, A. W. Hulskamp, T. Barlas, B. Marrant, G. A. M. van Kuik, D.-P. Molenaar, and M. Verhaegen. On the proof of concept of a smart wind turbine rotor blade for load alleviation. Wind Energy, 11(3):265-280, 2008. 


\section{Paper E}

Results from the first full scale wind turbine equipped with trailing edge flaps

Paper published in the proceedings of the 28th AIAA Applied Aerodynamics Conference, June 28 - July 1, 2010, Chicago, IL, USA. 


\title{
Results from the first full scale wind turbine equipped with trailing edge flaps
}

\author{
D. Castaignet* and J. J. Wedel-Heinen ${ }^{\dagger}$ \\ Vestas Wind Systems A/S, Roskilde, Denmark \\ T. Kim ${ }^{\ddagger}$ and T. Buhl $\S$ \\ Risø DTU, National Laboratory for Sustainable Energy, Roskilde, Denmark \\ N. K. Poulsen \\ Technical University of Denmark, DTU Informatics, Lyngby, Denmark
}

\begin{abstract}
The following paper describes a part of an experimental work carried out in the research project ATEF. This project is a collaboration between Vestas Wind Systems A/S, Risø DTU and DTU - Technical University of Denmark. The aim of the project is to test and validate trailing edge flap systems for load alleviation for wind turbines. The present work covers the first measurements of a trailing edge system ever to be carried out on a full scale turbine. The measurements and the potential load reduction are compared to simulations using aeroelastic tools. These aeroelastic models are tuned to the measurements without flaps. The results show a good agreement between measurements and simulations.
\end{abstract}

\section{Nomenclature}

\author{
$\alpha \quad$ Angle of attack $[\mathrm{rad}]$ \\ $\beta \quad$ Trailing edge flap angle [rad] \\ $\Delta C_{l}(\alpha, \beta)$ Extra lift coefficient of the airfoil due to the trailing edge flap \\ $\Delta C_{m}(\alpha, \beta)$ Extra moment coefficient of the airfoil due to the trailing edge flap \\ $\omega \quad$ rotor speed $[\mathrm{rad} / \mathrm{s}]$ \\ $M_{x}^{i}(t)$ Blade $i$ root flap moment $[\mathrm{kNm}]$ \\ BEM Blade Element Momentum \\ HAWC2 Horizontal Axis Wind Turbine Code \\ TEF Trailing Edge Flaps \\ VTS Vestas Turbine Simulator
}

\section{Introduction}

A wind turbine is subject to fluctuating loads in both normal operation and during extreme events due to turbulence, wind shear, tower shadow, wind gust, emergency shutdown etc. These fatigue and extreme loads increase significantly as the rotor size increases. A large part of modern wind turbines is Multi-Megawatt and has a rotor diameter larger than 90 meters. All the turbine components, such as blades, tower, shaft or gearbox are designed in order to sustain these loads for many years. Their costs are then highly dependent on the magnitude of the loads they face.

\footnotetext{
${ }^{*}$ PhD student, Global Research, Frederiksborgvej 399, Building 115, 4000 Roskilde, Denmark.

$\dagger$ Project Manager, Global Research, Frederiksborgvej 399, Building 115, 4000 Roskilde, Denmark

${ }^{\ddagger}$ Research Scientist, Wind Energy Department, Frederiksborgvej 399, Building 118, 4000 Roskilde, Denmark.

$\S$ Senior Scientist, Wind Energy Department, Frederiksborgvej 399, Building 118, 4000 Roskilde, Denmark.

ฯAssociate Professor, DTU Informatics, Anker Engelunds Vej 1, 2800 Lyngby,Denmark.
} 
Some modern Megawatt size wind turbines use cyclic pitch or individual pitch control in order to alleviate loads from wind shear or yaw error. ${ }^{1,2}$ Blade flap fatigue loads can be reduced by $28 \%$ according to Larsen et al. ${ }^{1}$ Since 2003 trailing edge flap systems have been studied as a possible way to alleviate even more loads. ${ }^{3,4}$ The pitch system is a device which controls the flow around the whole blade. This system can be too slow to react to flow disturbances because of the size of the blades and their inertia. However, trailing edge flaps control the flow locally, where wind turbulence has the most impact. It is also possible to alleviate other loads using trailing edge flaps because they can act at a higher frequency than the pitch system. Several studies show that up to $60 \%$ of load reduction could be achieved by using flaps. ${ }^{5}$ Wind tunnel experiments have been made with a $2 \mathrm{D}$ section of a blade equipped with active trailing edge flaps. They confirmed the potential benefit from such devices. ${ }^{6}$

For the first time, flaps are mounted on a full scale turbine. The Vestas V27 wind turbine with trailing edge flaps is used to test the possibility for load alleviation for wind turbines and to compare results between experiment and numerical analysis. It is the first attempt to compare analytical results with a full scale turbine equipped with trailing edge flaps.

\section{Prototype wind turbine: Vestas V27}

The test wind turbine is a Vestas V27, located at Ris $\varnothing$ DTU, National Laboratory for Sustainable Energy. The turbine design was developed in the eighties. It is a pitch controlled horizontal axis wind turbine, with a nominal power of $225 \mathrm{~kW}$, and 13 meter long blades.

Only one of its 3 blades is equipped with trailing edge flaps. Blade 1 refers to the blade with trailing edge flaps, while blades 2 and 3 are the original blades. There are 3 independent flexible flaps on blade 1 , their total length is $15 \%$ of the blade length (Figure 1). Several sensors have been added on the turbine for the purpose of the experiment: strain gauges and accelerometers on the blades, the shaft, the nacelle and on the tower. Pitot tubes, which are able to measure the local relative wind speed and the local angle of attack, are available for each flap. A meteorological mast is located 70m west of the Vestas V27. It measures the wind speeds and directions at different heights. The test site is located on the seaside: the sea is west of the wind turbine, some fields are east of the turbine, some buildings and a small village are north and south of the turbine. This gives very different wind conditions depending on the wind direction. A bigger turbine is located 100m south of the Vestas V27: the V27 can also be tested in wake conditions.

The tests reported in this paper do not involve any controller on the trailing edge flaps. The flaps are actuated either at a fixed position, or at a fixed frequency. A low level control ensures the actual flap angle matches the requested angle, independent of the aerodynamic loads on the flap. The flap actuator test described in this paper is limited to a few degrees.

The Vestas V27 turbine is a fixed speed turbine equipped. It has two generator modes resulting in two rotor speed: the rotor speed is 33rpm at low wind speeds and 43rpm at higher wind speeds.

\section{Numerical Analysis}

In this paper, numerical analysis is carried out with two different aeroelastic tools: the Vestas Turbine Simulator (VTS) and the Horizontal Axis Wind Turbine Code (HAWC2). VTS is a Vestas version of Flex5, developed by DTU Mechanical Engineering. It is a state-of-the art Blade Element Momentum (BEM) code based on the modal approach. Both blades and tower are flexible, modeled by as many mode shapes as required. It has all the usual engineering models used in the wind turbine simulation tools, such as PrandtlGlauert tip correction, dynamic wake model, turbulent wind, oblique inflow model etc. HAWC2 also includes the mentioned models but is a nonlinear multibody finite element code. ${ }^{7}$

The dynamic stall model originally coded in Flex5 works only for rigid blade sections. ${ }^{8}$ The implementation of the trailing edge flaps in VTS is based on the code written by P. B. Andersen and M. Gaunaa. ${ }^{9,10}$ It is an extension of Theodorsen's work ${ }^{11}$ which includes a Beddoes-Leishmann type dynamic stall model. ${ }^{12}$ The model uses lookup tables of the static lift and moment coefficients of an airfoil with a trailing edge flap at different angles of attack and flap angles. The model has been validated by comparison to wind tunnel measurements. ${ }^{9}$

In the traditional BEM code, a trailing edge flap disturbs the flow only at the section where the trailing edge flap is actuated. A near wake model is implemented in $\mathrm{HAWC} 2^{13}$ in order to model the radial interaction between the sections of the blade. These 3D effects are particularly important to model the flow around the 


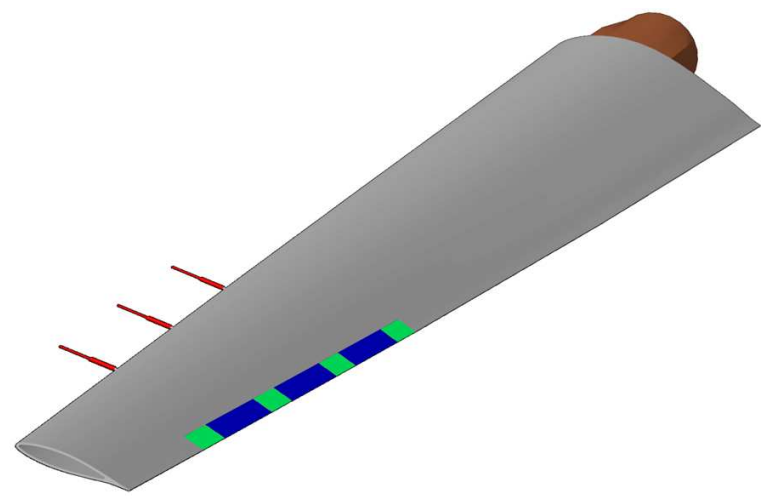

(a)

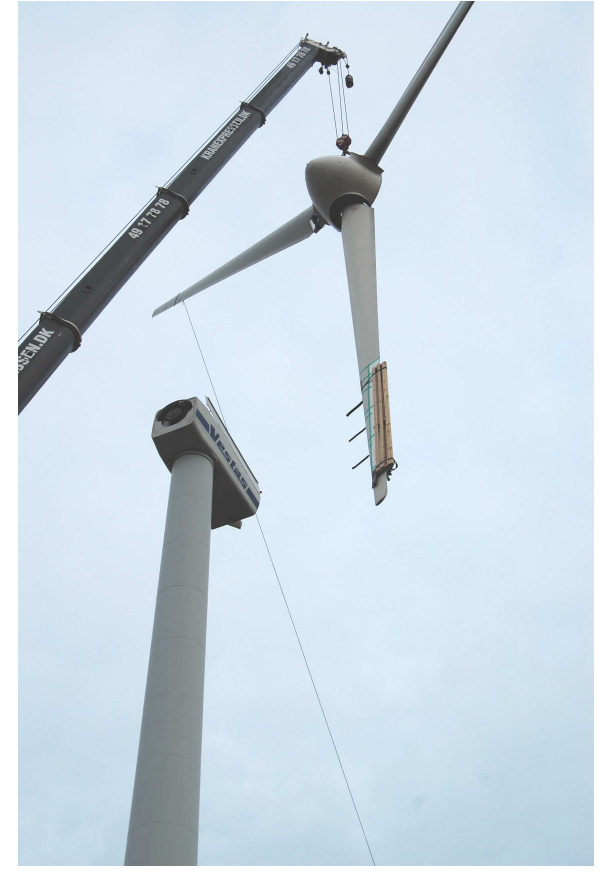

(b)

Figure 1. (a) Illustration of the Vestas V27 blade equipped with trailing edge flaps and Pitot tubes. Red: Pitot tubes, Blue: active trailing edge flaps, Green: dummy flaps. (b) The rotor with TEF is being installed on the Vestas V27.

tips of a trailing edge flap.

\section{Results}

The purpose of the measurements described in this paper is to validate the models used in the simulations and to confirm the potential of the flaps in alleviating loads on different components of the turbine. Three types of measurements were run:

Measurements A The trailing edge flaps are fixed in their neutral positions.

Measurements B The trailing edge flaps are fixed alternating in a low lift and a high lift configuration.

Measurements C The trailing edge flaps are actuated at a given frequency.

Measurements from the sensors on the meteorological mast and on the turbines are saved in 10 minute series. Simulations of the V27 turbine are run with winds which have the same mean speed, turbulence intensity, direction and shear as observed during the 10 minute measurements. Spectral densities and averaged values of the measurements and the simulations with the same wind properties can be compared.

The efficiency of the trailing edge flaps can be estimated by two different ways: by comparing the root moments of the blade with the active TEF with the two other blades, or by comparing the root moments of the blades when TEF are active and when they are not active. Both methods have been used.

\section{IV.A. Measurements A: Flaps fixed in their neutral configuration: 0 degrees}

The structural response of blade 1 differs from the 2 other blades because of the structural modifications made to fit the TEF: the weight and the edgewise stiffness of blade 1 were altered. Running measurements and simulations with TEF fixed at their neutral position is then useful to check the tuning of the model for the 3 blades, and to check that the eigenfrequencies of blade 1 has not been altered too much.

Spectral densities of the measured flap and edge blade root moments of the three blades in Figure 2 show clearly the $1 \mathrm{P}, 2 \mathrm{P}$ and $3 \mathrm{P}$ frequencies. The $1 \mathrm{P}$ frequency corresponds to one event per rotor revolution, the 
$2 \mathrm{P}$ and $3 \mathrm{P}$ frequencies are the double and the triple of the $1 \mathrm{P}$ frequency. These frequencies are excited by events which depend on the azimuth angle of the rotor such as wind shear, tower shadow, or yaw error. Two more peaks are observed: one has a frequency close to $4 \mathrm{P}$, the other one close to $5 \mathrm{P}$. They correspond respectively to the flapwise and the edgewise eigenfrequencies of the blades.

TEF on blade 1 made the blade heavier and less stiff edgewise than the two other blades. Its edgewise eigenfrequency is then expected to be lower than for the two other blades, which is observed by the measurements in Figure 2. Both VTS and HAWC2 models are tuned for the prototype turbine, and this difference in edgewise eigenfrequencies is modeled in the VTS and the HAWC2 model of the V27 (Figures 3 and 4). Figure 4 shows that the spectral densities of the blade root flap and edge moments are well reproduced in the simulations. Minor differences remain. The first edgewise eigenfrequency of blade 1 is a bit higher in the simulations than in the measurements. The peak in the blade root edge moment at the $2 \mathrm{P}$ frequency does not show as sharp in the HAWC2 simulations than in the VTS simulations and the test turbine measurements. These VTS and HAWC2 models are used to simulate the V27 turbine with active flaps in the next sections.

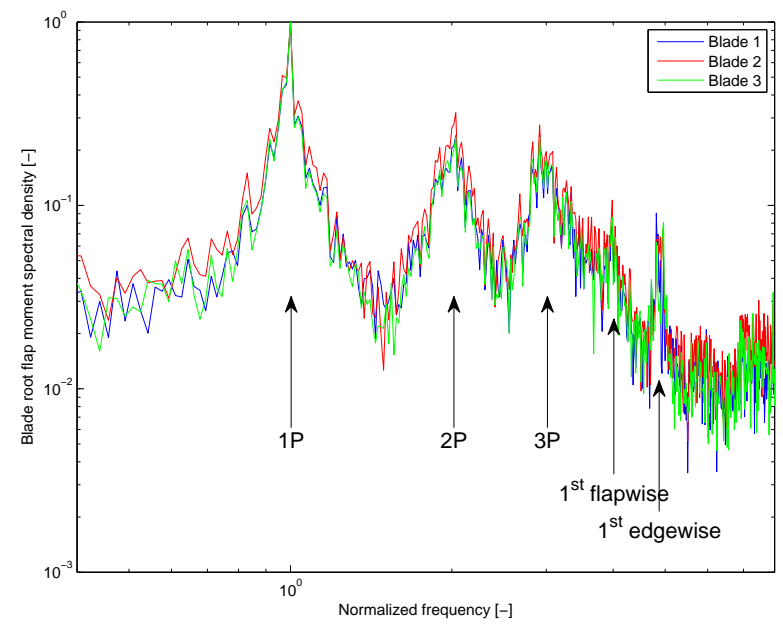

(a) Flap bending moment

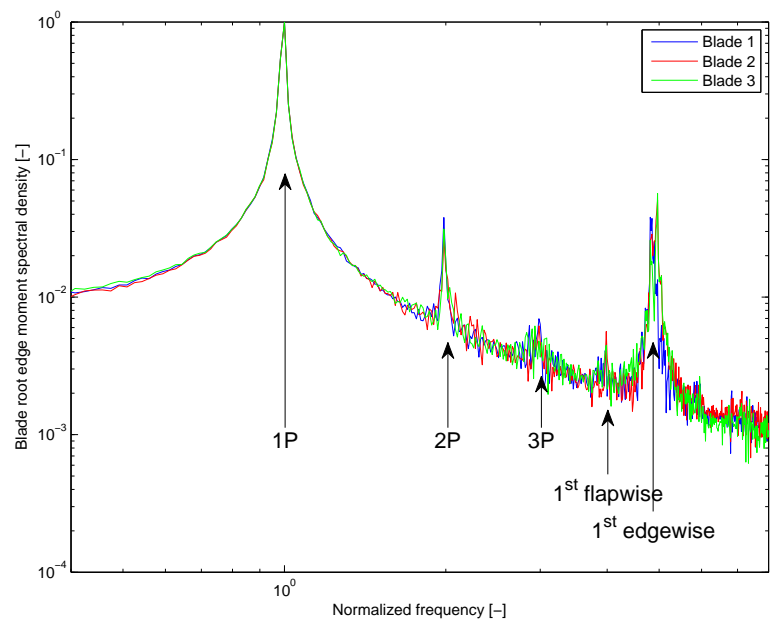

(b) Edge bending moment

Figure 2. Measurements. Spectral density of the measured blade root bending moments for the 3 blades when the TEF are fixed in their neutral position. Frequencies are normalized with respect to the $1 \mathrm{P}$ frequency. The power spectral densities are also normalized.

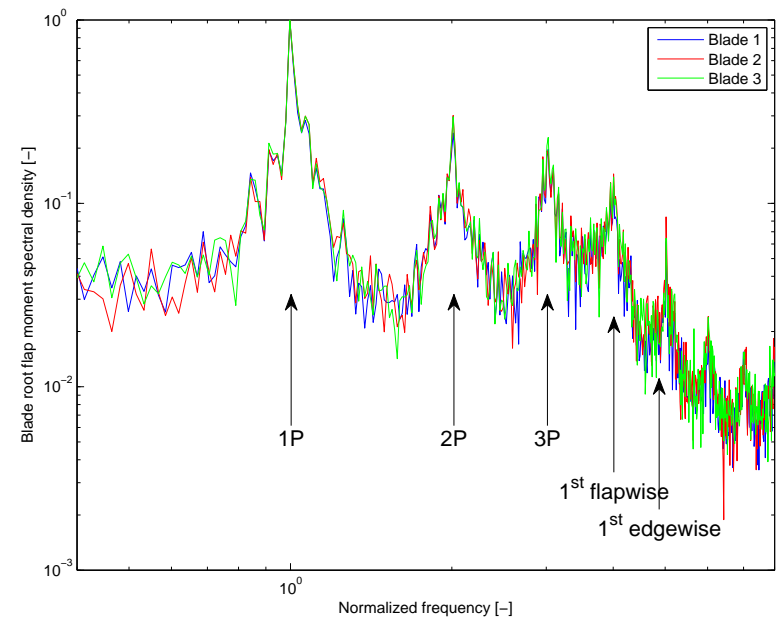

(a) Flap bending moment

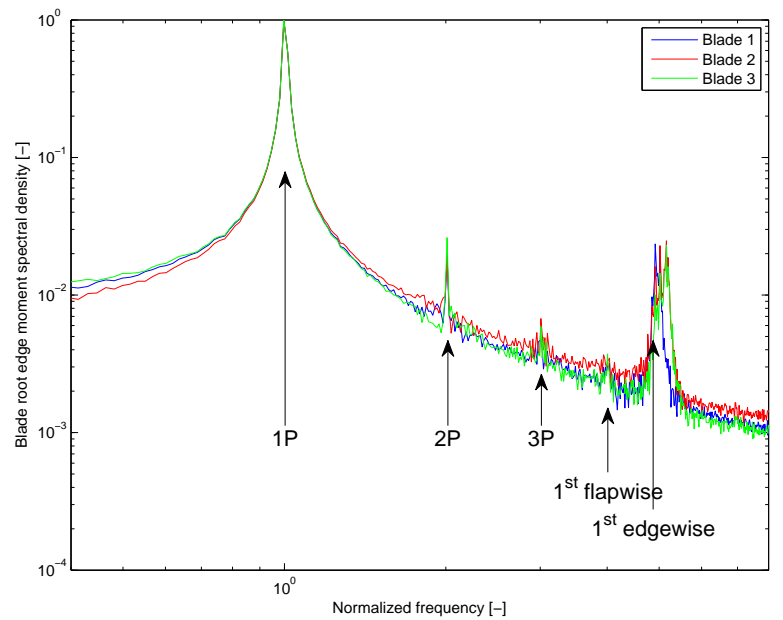

(b) Edge bending moment

Figure 3. VTS simulations. Spectral density of the simulated (VTS) blade root bending moments for the 3 blades when the TEF are fixed in their neutral position. Frequencies are normalized with respect to the 1P frequency. The power spectral densities are also normalized. 


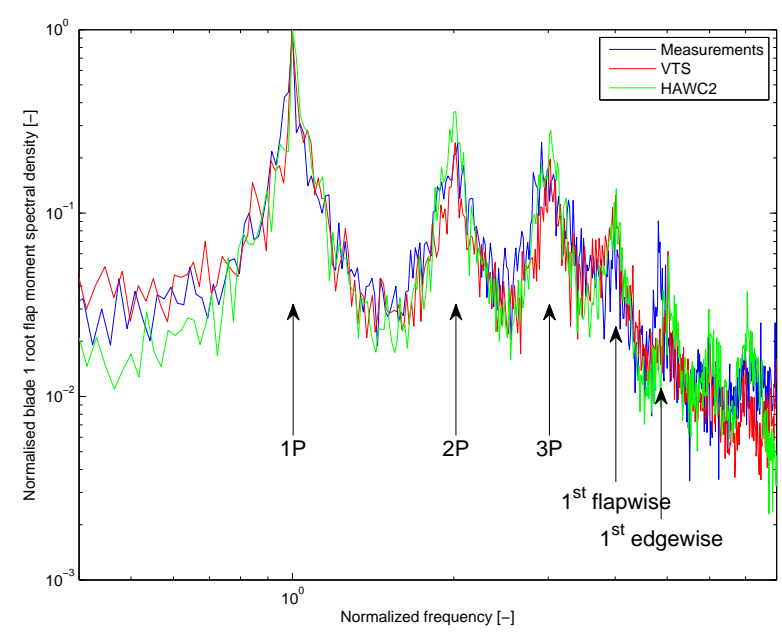

(a) Flap bending moment

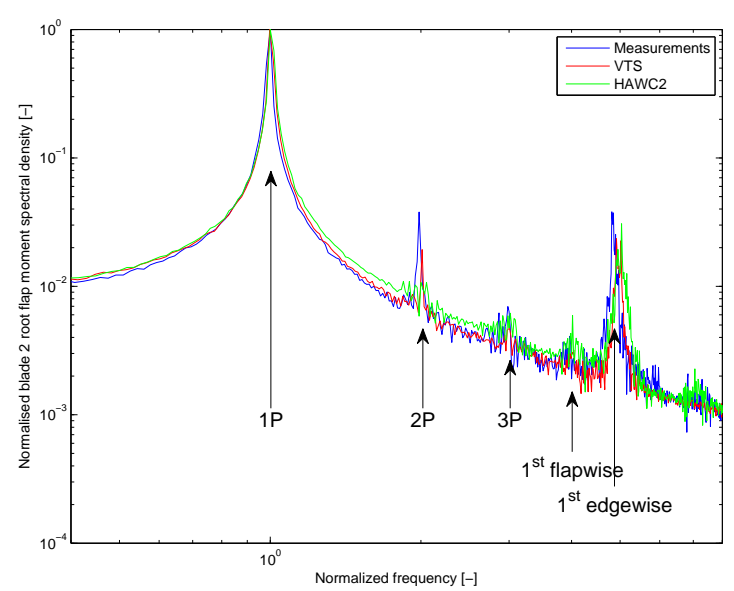

(b) Edge bending moment

Figure 4. Measurements, HAWC2 and VTS simulations. Comparison of the spectral densities of the simulated (VTS and HAWC2) and the measured blade root bending moments for blade 1 when the TEF are fixed in their neutral position. Frequencies are normalized with respect to the $1 \mathrm{P}$ frequency. The power spectral densities are also normalized.

\section{IV.B. Measurement B: Flaps fixed alternating in a low lift and a high lift configuration}

Blade root flap moment varies depending on both time and azimuth angle because of:

- slow and periodic phenomena like wind shear, tower shadow or yaw error

- fast and stochastic phenomena like wind turbulence

- actuation of TEF.

Due to the limited capacity of the prototype system, the effect of TEF on the blade root flap moment is lower than the effect of wind shear, yaw error or wind turbulence. It makes the detection of the effect of the TEF on the blade root flap moment difficult. It can nevertheless be clearly seen when the blade root flap moment of blade 1 is compared to the blade root flap moments of the two other blades (Figure 5). These signals are shifted and filtered in order to highlight the change in average blade root bending moment.

The difference of flap moments is calculated as

$$
\begin{aligned}
& \triangle_{1,2}(t)=M_{x}^{1}(t)-M_{x}^{2}\left(t+\frac{2 \pi}{3 \omega}\right) \\
& \triangle_{1,3}(t)=M_{x}^{1}(t)-M_{x}^{3}\left(t-\frac{2 \pi}{3 \omega}\right)
\end{aligned}
$$

where $M_{x}^{i}$ is the root flap moment of blade $i$ and $\omega$ is the rotor speed. $\frac{2 \pi}{3 \omega}$ corresponds to the time for a blade to rotate $2 / 3$ of a rotation. The difference $\triangle_{1,2}(t)$ is the difference of flap moment between blade 1 and blade 2 when the blades have the same azimuth angle. The impact of azimuth dependent phenomena on this difference is then lowered, and the impact of TEF is highlighted. The signals $\triangle_{1,2}(t)$ and $\triangle_{1,3}(t)$ are filtered with a low pass zero-phase digital filter above $0.1 \mathrm{~Hz}$ in order to filter the high frequencies which are the consequences of the wind turbulence, the wind shear and the tower shadow. These filtered signals $\tilde{\triangle}_{1,2}(t)$ and $\tilde{\triangle}_{1,3}(t)$ are divided by the mean flap root moment in order to normalize them and are plotted in Figure 5.

A negative TEF angle decreases the lift coefficient over the airfoil (low lift configuration), resulting in a decrease of the blade root flap moment. In average, when TEF are fixed in their low lift configuration, the blade 1 flap root moment is lower than both the two other blade root moments. The opposite behavior is observed when the TEF are in their high lift configuration. Figure 5 (b) clearly shows that actuating the TEF from their low lift to high lift configuration has an influence on the average blade root flap moment. The tests described in this paper includes flap motions that alter the blade root flap moment by 1 to $2 \%$ of the mean flap moment. 
Simulations are run in VTS and HAWC2 with the same wind conditions and TEF actuation as in the measurements. The same postprocessing is applied to the simulated blade root flap moments. As expected, the same trend of the difference in blade root flap moments is observed (Figure 5 (a)). Both aeroelastic codes show the same efficiency of the TEF in the blade root flap moments: the TEF are able to alter the flap blade root moment by approximately 2 to $3 \%$ of the mean flap blade root moment. This is more than what is measured on the prototype turbine.

The aerodynamic model used in both VTS and HAWC2 is based on lookup tables for the static lift and moment changes due to the TEF at a given angle of attack $\alpha$ and a given TEF angle $\beta\left(\Delta C_{l}(\alpha, \beta)\right.$ and $\left.\Delta C_{m}(\alpha, \beta)\right)$. There is an uncertainty on these values because wind tunnel tests with the same profile and the same trailing edge flaps as on the prototype turbine have not yet been carried out. Values used come from CFD simulations on a Ris $\varnothing$ profile. Having a flexible flap increases the uncertainty because it is not possible to control the shape of the whole TEF under various loads: the TEF may deform under high pressure loads, and lose their efficiency.
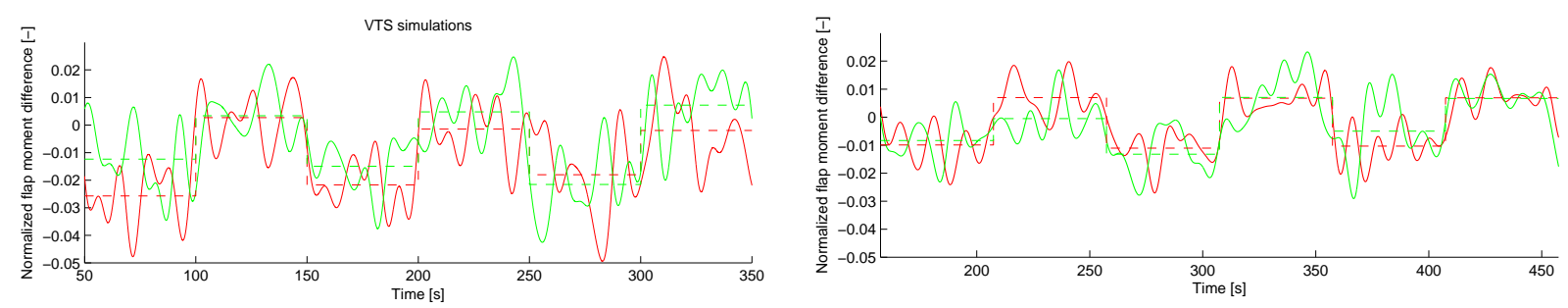

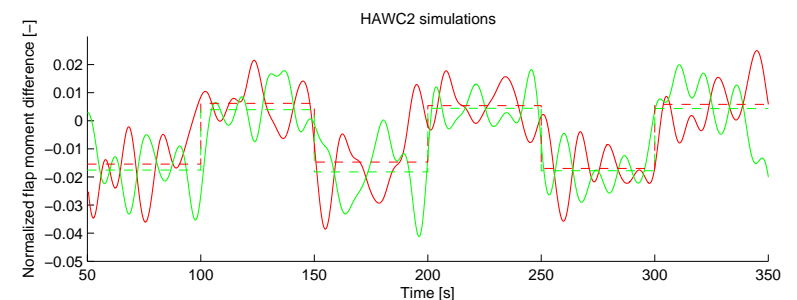

(a) VTS (top) and HAWC2 (bottom) simulations

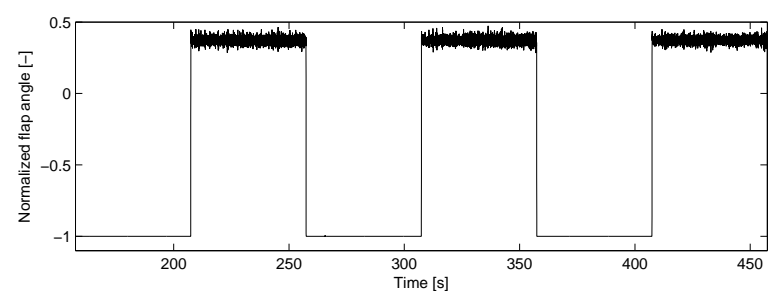

(b) Measurements

Figure 5. (a) Simulations. (b) Measurements. Difference in flapwise blade root moment between blade 1 and 2 (red plot) and blade 1 and 3 (green plot). The differences $\tilde{\triangle}_{1,2}(t)$ and $\tilde{\triangle}_{1,3}(t)$ are calculated according to 1 and filtered with a low-pass zero-phase filter above $0.1 \mathrm{~Hz}$. The dashed lines are averaged values of the differences over the 50s where the flaps are in low or high lift position.

\section{IV.C. Measurements C: Flaps active at a fixed frequency}

Another set of measurements consist in actuating the TEF at a given frequency, close to the first flapwise eigenfrequency of the blade (around 3.7 times the $1 \mathrm{P}$ frequency). When the measurements were taken, the meteorological mast was in the wake of the turbine: measurements of the wind made by the mast were thus disturbed by the turbine. It was not possible to get accurate values of the wind characteristics (mean wind speed, wind shear, turbulence intensity), which explains why measured and simulated spectral densities differ (Figure 8).

As expected, both measurements (Figure 6) and simulations (Figure 7) show a response in the flap bending root moment of blade 1 at the same frequency as the flap was activated. The response is also seen in blade 2 and 3, with a much lower amplitude, because of the coupling between the three blades through the hub. In both measurements and simulations, the amplitude of the peak in the PSD of the blade root flap moment at the TEF frequency is similar to the peaks at $2 \mathrm{P}$ and $3 \mathrm{P}$ frequencies. It seems that the HAWC2 and VTS models are more accurate when TEF are actuated at this frequency than when TEF are static. More measurements are nevertheless needed in order to confirm this conclusion.

Measurements also show that actuating the TEF of blade 1 at 3.7 times the $1 \mathrm{P}$ frequency results in the excitation of blades 2 and 3 at close to 5 times the $1 \mathrm{P}$ frequency (Figure 6). Figure 9 shows that these excitations correspond mainly to edgewise vibrations of blade 2 and 3, and their frequency is the edgewise eigenfrequency of the blade. They were not predicted at such a high amplitude by neither HAWC2 nor VTS (Figures 7 and 8). The flapwise mode of the blades has a high aerodynamic damping, whereas the edgewise 
mode is lightly damped. That's why the flapwise excitation of blade 1 couples to an edgewise excitation of blades 2 and 3, at the edgewise eigenfrequencies of the blades. What is called the blade edgewise mode is not a pure vibration of the blade in the edgewise direction. It couples with the modes of the other components of the turbine, including the blade flapwise mode. That's why these vibrations are also clearly monitored by the strain gauges which measure the flapwise bending moments of the blade.

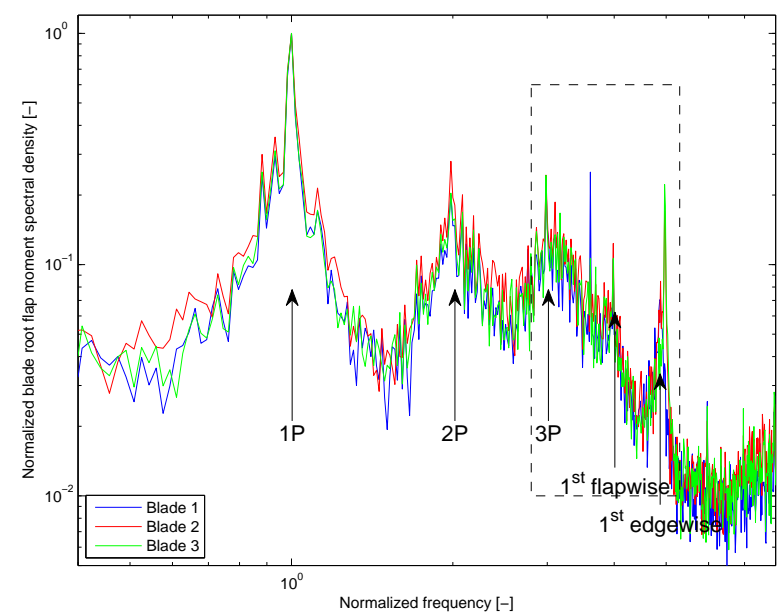

(a)

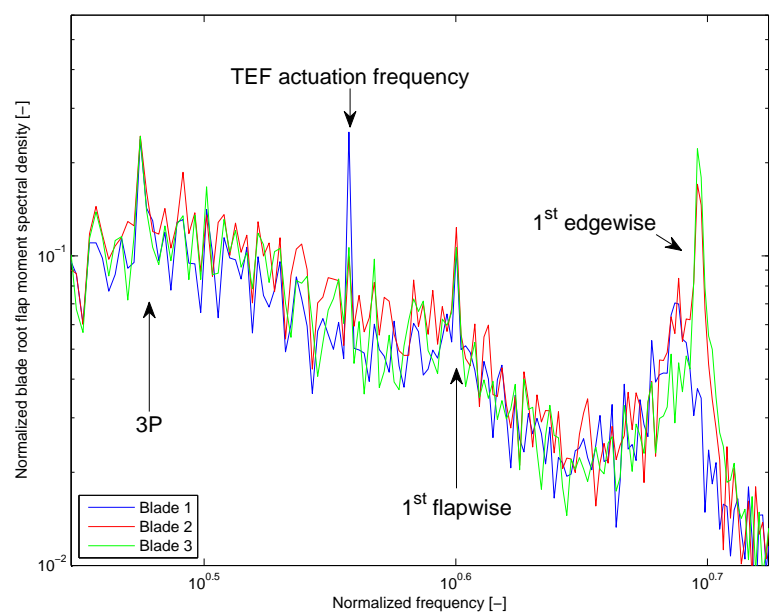

(b)

Figure 6. Measurements. Spectral density of the measured blade root bending moments for the 3 blades when the TEF are actuated at a fixed frequency. Frequencies are normalized with respect to the $1 P$ frequency. The power spectral densities are also normalized. (b) is a zoom over the dashed square in figure (a).

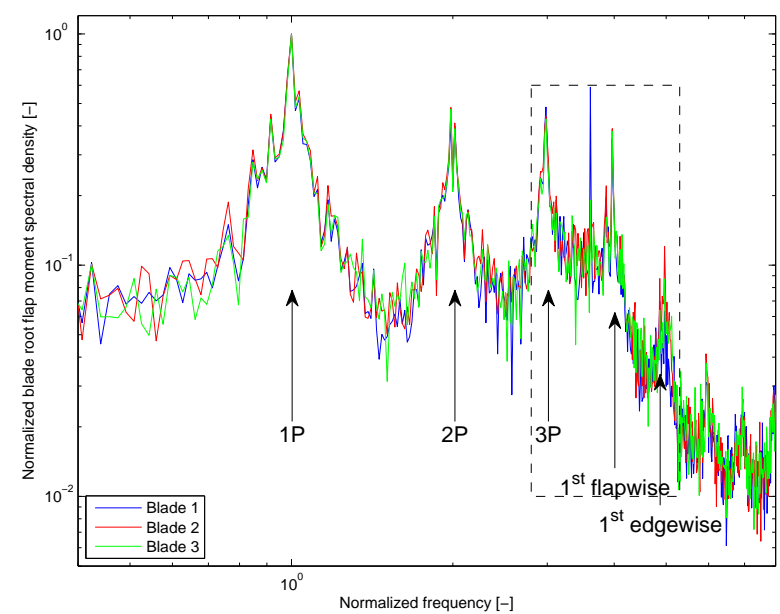

(a)

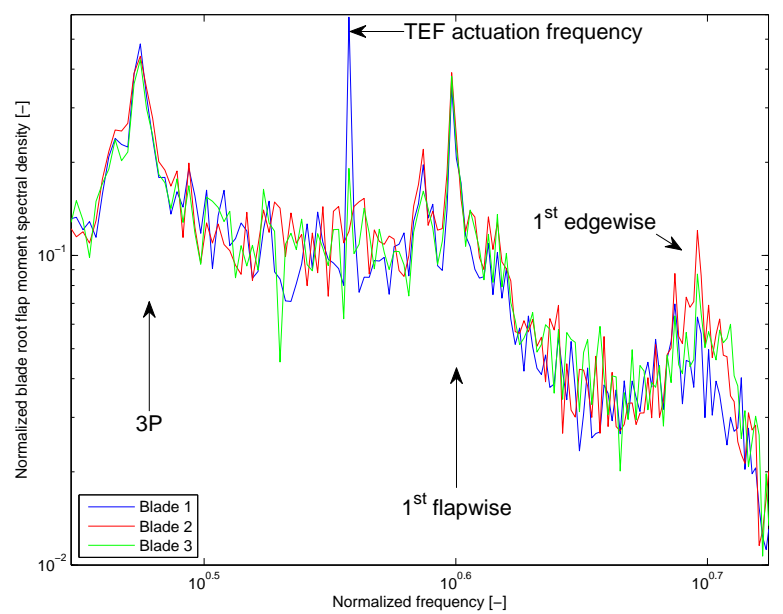

(b)

Figure 7. VTS simulations. Spectral density of the simulated (VTS) flap blade root bending moments for the 3 blades when the TEF are actuated at a fixed frequency. Frequencies are normalized with respect to the $1 P$ frequency. The power spectral densities are also normalized. (b) is a zoom over the dashed square in figure (a).

\section{Conclusion}

A Vestas V27 wind turbine has been equipped with trailing edge flaps on one blade. Measurements were run with different flap actuation and results from aeroelastic simulations with both VTS and HAWC2 were compared with these measurements.

First the trailing edge flaps were fixed in their neutral position in order to confirm that the eigenfrequencies of the altered blade are similar to those of the two other blades. These measurements also helped tuning the VTS and the HAWC2 models. Then the effect of the trailing edge flaps on the blade root moments have been

$$
7 \text { of } 9
$$




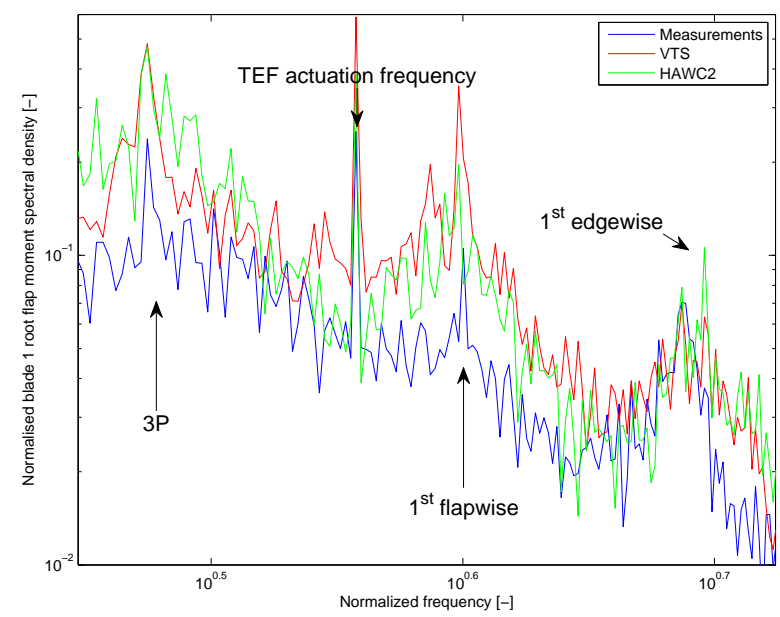

(a) Blade 1

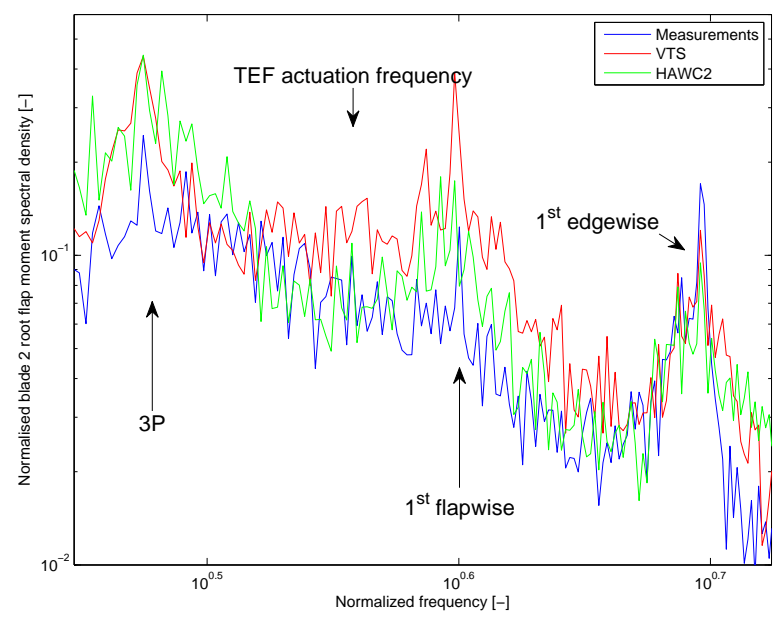

(b) Blade 2

Figure 8. Measurements, VTS and HAWC2 simulations. Comparison of the spectral densities of the measured and simulated (VTS and HAWC2) flap blade root bending moments for the blades 1 (a) and 2 (b) when the TEF are actuated at a fixed frequency. Frequencies are normalized with respect to the $1 P$ frequency. The power spectral densities are also normalized.

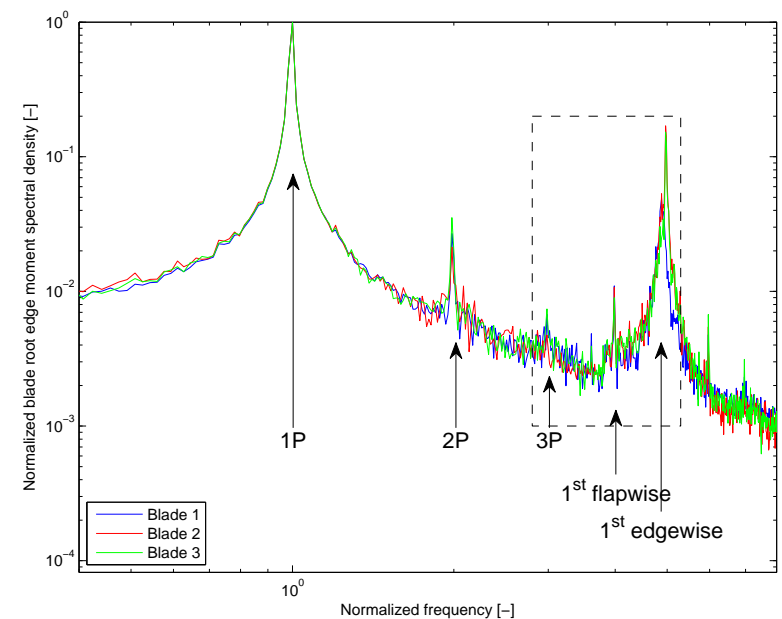

(a)

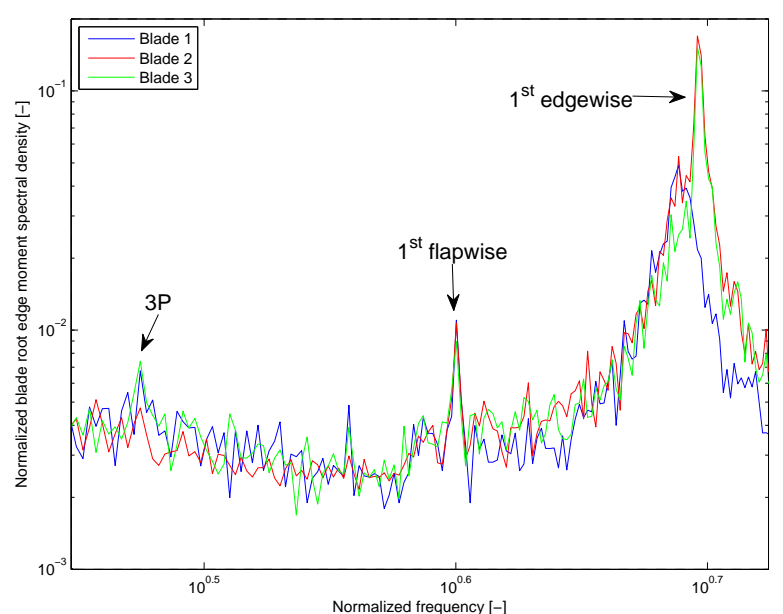

(b)

Figure 9. Measurements. Spectral density of the measured edge blade root bending moments for the 3 blades when the TEF are actuated at a fixed frequency. Frequencies are normalized with respect to the $1 \mathrm{P}$ frequency. The power spectral densities are also normalized. (b) is a zoom over the dashed square in figure (a). 
studied. The trailing edge flaps were fixed alternating in their high lift and in their low lift configuration, by sequence of 50 seconds. Measurements show that the average blade root flap moment of blade 1 is clearly lower than the average blade root flap moment of the 2 other blades when the trailing edge flaps are in their low lift configuration. The measured average blade 1 root flap moment is less than $1 \%$ higher or lower than the measured flap moment of blades 2 and 3 depending on the position of the trailing edge flaps. Simulations with both HAWC2 and VTS predict a slightly higher impact of the trailing edge flaps on the blade root flap moment. The last set of measurements covered actuation of the trailing edge flaps at a fixed frequency. Simulations predict well the increase in the power spectral density of the blade root flap moment of blade 1 at this frequency. But measurements show that actuating the trailing edge flaps on blade 1 results in edgewise vibrations in the 2 other blades, at a higher frequency than the flaps frequency. This coupling can not be seen in the simulated results.

\section{Acknowledgments}

The ATEF project is partially funded by the Danish National Advanced Technology Foundation (Højteknologifonden).

The authors would like to acknowledge R. Kjærsgaard, A. Vesth, A. B. Andersen and K. Enevoldsen from Ris $\emptyset$ DTU, and N. A. Olesen, P. Gooda, A. Olesen, E. Bolesen and C. Lupu from Vestas Wind Systems $\mathrm{A} / \mathrm{S}$ for their work setting up the test and for their valuable contribution.

\section{References}

${ }^{1}$ Larsen, T. J., Madsen, H. A., and Thomsen, K., "Active load reduction using individual pitch, based on local blade flow measurements," Wind Energy, Vol. 8, No. 1, 2005, pp. 67-80.

${ }^{2}$ Bossanyi, E. A., "Individual blade pitch control for load reduction," Wind Energy, Vol. 6, No. 2, 2003, pp. 119-128.

${ }^{3}$ Barlas, T. K. and van Kuik, G. A. M., "Review of state of the art in smart rotor control research for wind turbines," Prog Aerospace Sci Energy, 2009.

${ }^{4}$ Buhl, T., Gaunaa, M., and Bak, C., "Potential load reduction using airfoils with variable trailing edge geometry," Journal of Solar Energy Engineering, Vol. 127, November 2005, pp. 503-516.

${ }^{5}$ Andersen, P. B., Gaunaa, M., Bak, C., and Buhl, T., "Deformable trailing edge flaps for modern megawatt wind turbine controllers using strain gauge sensors," Wind Energy, Vol. 13, No. 2, 2010, pp. 193-206.

${ }^{6}$ Bak, C., Gaunaa, M., Andersen, P. B., Buhl, T., Hansen, P., and Clemmensen, K., "Wind tunnel test on airfoil Ris $\varnothing$-B1-18 with an Active Trailing Edge Flap," Wind Energy, Vol. 13, No. 2-3, 2010, pp. 207-219.

${ }^{7}$ Larsen, T. J., "How 2 HAWC2, the user's manual," Tech. Rep. Ris $\varnothing-\mathrm{R}-1597$, Ris $\varnothing$ DTU, May 2009.

${ }^{8} \varnothing$ ye, S., "Dynamic Stall simulated as time lag of separation," Tech. rep., Technical University of Denmark, Department of Fluid Mechanics.

${ }^{9}$ Andersen, P. B., Gaunaa, M., Bak, C., and Hansen, M. H., "A dynamic stall model for airfoils with deformable trailing edges," Wind Energy, Vol. 12, No. 8, 2009, pp. 734-751.

${ }^{10}$ Gaunaa, M., "Unsteady two-dimensional potential-flow model for thin variable geometry airfoils," Wind Energy, Vol. 13, No. 2-3, 2010, pp. 167-192.

${ }^{11}$ Theodorsen, T., "General Theory of Aerodynamical Instability and the Mechanism of Flutter," Tech. Rep. 496, NACA, 1935.

${ }^{12}$ Leishman, J. G., "Unsteady lift of a flapped airfoil by indicial concepts," Journal of Aircraft, Vol. 31, No. 2, March-April 1994, pp. 288-297.

${ }^{13}$ Andersen, P. B., Gaunaa, M., Zahle, F., and Madsen, H. A., "A near wake model with far wake effects implemented in a multi body aero-servo-elastic code," Proceedings of the European Wind Energy Conference EWEC 2010,2010, pp. 44-49. 


\section{Paper F}

Full-scale test of Trailing Edge Flaps on a Vestas V27 wind turbine. Active load reduction and system identification

Paper submitted at the Wind Energy journal.

This work has been submitted to the Wind Energy journal for possible publication. Copyright may be transferred without notice, after which this version will be superseded. 


\title{
Full-scale test of Trailing Edge Flaps on a Vestas V27 wind turbine. Active load reduction and system identification.
}

\author{
Damien Castaignet ${ }^{1}$, Thanasis Barlas ${ }^{2}$, Thomas Buhl ${ }^{2}$, Niels K. Poulsen ${ }^{3}$, Jens Jakob Wedel- \\ Heinen ${ }^{1}$, Niels A. Olesen ${ }^{1}$, Christian Bak ${ }^{2}$ and Taeseong Kim ${ }^{2}$. \\ ${ }^{1}$ Vestas Wind Systems A/S, Global Research \& Innovation, Roskilde, Denmark. \\ ${ }^{2}$ Risø DTU, National Laboratory for Renewable Energy, Roskilde, Denmark. \\ ${ }^{3}$ Technical University of Denmark, DTU Informatics, Lyngby, Denmark.
}

\begin{abstract}
A full-scale test was performed on a Vestas V27 wind turbine equipped with one active $70 \mathrm{~cm}$ long trailing edge flap on one of its $13 \mathrm{~m}$ long blades. Active load reduction could be observed in spite of the limited spanwise coverage of the single active trailing edge flap. Two proportional controllers based respectively on the flapwise blade root moment and on the local angle of attack, as well as a frequency-weighted model predictive control were tested succesfully on this demonstrator turbine. An average flapwise blade root load reduction of $14 \%$ was achieved with the model predictive control during a 38 minute test, and a reduction of $20 \%$ of the amplitude of the 1 P loads was measured. A system identification test was also performed and an identified linear model, from trailing edge flap angle to flapwise blade root moment was derived and compared to the linear analytical model used in the model predictive control design model. Flex 5 simulations run with the same model predictive control showed a good correlation between the simulations and the measurements in terms of flapwise blade root moment spectral densities, in spite of significant differences between the identified linear model and the model predictive control design model. Copyright (c) 2010 John Wiley \& Sons, Ltd.
\end{abstract}

\section{KEYWORDS}

trailing edge flaps; active load reduction; system identification; full-scale test

\section{Correspondence}

D. Castaignet, Vestas Wind systems A/S, Frederiksborgvej 399, DK-4000, Roskilde, Denmark.

E-mail:daca@vestas.com

\section{Contract/grant sponsor}

This work was supported by the Danish National Advanced Technology Foundation (Højteknologifonden), as part of the ATEF (Adaptive Trailing Edge Flaps) project.

Received ...

\section{INTRODUCTION}

Wind turbines rotor size has increased significantly over the last years, with the leading wind turbine manufacturers marketing turbines with rotor diameters over $100 \mathrm{~m}$. Increasing the rotor diameter is an effective way to increase the power production of the turbine, but it comes at the cost of higher fatigue and extreme loads on all the turbine components. Reducing those loads are necessary in order to keep reducing the cost of wind energy.

Loads in a wind turbine have different sources: wind conditions, such as wind turbulence or wind shear, tower shadow, resonance of wind turbine modes, yaw misalignment of the turbine, emergency shutdowns, grid loss etc [13]. Some modern wind turbines use cyclic pitch or individual pitch in order to alleviate some of the loads. Cyclic pitch, which originates from helicopter control, consists in pitching the three blades with a 120 degrees phase shift. It is used to alleviate tilt and yaw loads on the rotor. In individual pitch control (IPC), each blade can pitch independently of the two other blades. IPC is one of the most advanced active control to alleviate loads in today's turbines [10, 11, 23, 29, 21], but its performance is limited by the relatively slow pitch actuators and by the necessity to limit pitch actuation in order to preserve the actuators and the blade bearings. Some passive load controls, like prebent blades, swept blades $[4,20]$ or bending/twist-coupled blades $[28,14]$, show also promising load reductions.

In order to reduce even further and in a more efficient way the loads on the rotor, several concepts of "smart" rotors have been investigated. A "smart" rotor consists in distributed sensors (accelerometers, strain gauges, Pitot tubes, pressure tabs etc.) and actuators (trailing edge flaps, microtabs, boundary layer suction or blowing jets, plasma actuators etc.) along the blades. A detailed overview of different "smart" rotor concepts is given in [6].

Trailing edge flaps on turbine blades have been investigated for several years, as part of this "smart" rotor 
concept. CFD simulations [9], 2D aeroelastic simulations $[7,12]$ and $3 \mathrm{D}$ aeroelastic simulations $[15,2,16]$ confirmed the high potential of trailing edge flaps to reduce flapwise blade root fatigue loads. Wind tunnel tests on a blade section $[5,33,1]$ as well as on a scaled turbine [32] corroborated the ability of the trailing edge flaps to reduce loads. At last, in 2010, a full scale test was carried out on the Vestas V27 turbine located at Ris $\varnothing$ DTU. Only open loop controls were tested at that time, and no active fatigue load reduction was performed [18].

This paper shows the results from the latest tests made in 2011 on the same Vestas V27 demonstrator turbine. Those tests include active load reduction achieved with proportional controllers and with a frequency weighted model predictive control [17], and derivation of a time invariant linear model, from trailing edge flap angle to flapwise blade root moment, derived with a system identification method [22, 31].

The first section of this paper describes the demonstrator wind turbine. The tests performed during this test campaign are developed in the second section, and the results from the field test are presented in Section 4. Finally, the field results are compared with some Flex5 simulations in Section 5.

\section{EXPERIMENTAL SETUP}

\subsection{V27 wind turbine}

The demonstrator turbine for the ATEF (Adaptive Trailing Edge Flaps) project is the Vestas V27 wind turbine located at Ris $\varnothing$ DTU, National Laboratory for Renewable Energy (Figure 1). The V27 turbine is an horizontal axis wind turbine, with a nominal power of $225 \mathrm{~kW}$, and a rotor diameter of $27 \mathrm{~m}$.

The collective pitch of the turbine is used for regulating the power production and limit the thrust. No modern variable speed loads control is implemented in the V27 turbine.

Designed in the eighties, the Vestas V27 is however similar to modern multi-megawatt wind turbines on some aspects: it is a pitch controlled wind turbine and its blade tip speed is similar to the one of a modern turbine at rated power.

On the other hand, compared to modern turbines, the V27 rotor speed is higher and its structure, especially its blades, are much stiffer. The frequencies of interest (the $1 \mathrm{P}$ frequency, corresponding to one event per rotor revolution, and the lowest turbine eigenfrequencies) are thus all larger than for bigger turbines. As a consequence, the trailing edge flaps are actuated at higher frequencies on the V27 wind turbine than they would be on a multimegawatt turbine. Another important difference: the V27 turbine works at two constant rotor speeds, $32 \mathrm{rpm}$ at wind speeds lower than 4 to $5 \mathrm{~m} . \mathrm{s}^{-1}$ and $43 \mathrm{rpm}$ at higher wind speeds. Modern turbines run at variable speed and keep a more constant tip speed ratio.

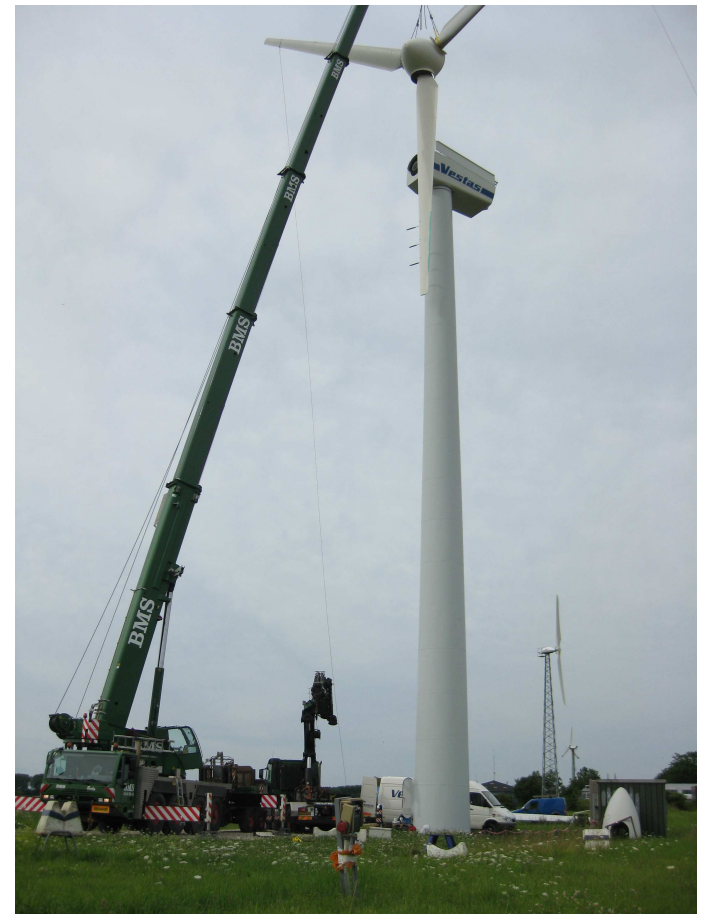

Figure 1. Photo of the V27 wind turbine demonstratror while the rotor with trailing edge flaps is being mounted.

\subsection{Instrumentation}

Three trailing edge flaps, with a spanwise length of $70 \mathrm{~cm}$ each and a chord ratio between 13 and $18 \%$, are mounted on one of the V27 original blades, but the tests presented in this paper were run with only one of them, the outer one. So only $5 \%$ of the blade span was covered with a working flap. The trailing edge flaps are stiff hinged flaps. The whole actuator system is mounted inside the blade in order not to disturb the flow around the aerofoil. The trailing edge flaps were strong enough to be actuated at the $1 \mathrm{P}$ frequency (between 0.5 and $0.7 \mathrm{~Hz}$ ), at full amplitude, with a negligible time lag. They could also be actuated at frequencies up to $4 \mathrm{P}$, but at a reduced amplitude and with a reduced accuracy.

The demonstrator turbine is equipped with several extra sensors. The sensors available to the trailing edge flap controller include strain gauges at the root of the modified blade, flapwise and edgewise, two 3D accelerometers at the tip of the blade, three 5-hole Pitot tubes and three pairs of pressure tabs at the leading edge of the three trailing edge flaps, position sensors on the three trailing edge flaps, and a pitch position sensor. The pitch position sensor was unfortunately not functional during the tests. Other sensors, not available to the trailing edge flaps controller but logged for postprocessing, include strain gauges at the root of the two other blades, flapwise and edgewise, a 3D accelerometer in the nacelle, strain gauges at the tower bottom and a nacelle cup anenometer. Data from a meteorological mast, located $70 \mathrm{~m}$ west of the wind 


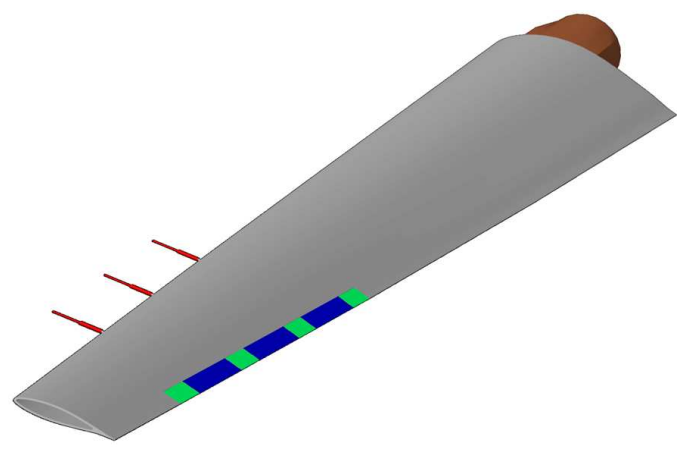

Figure 2. Illustration of the Vestas V27 blade equipped with trailing edge flaps (blue), Pitot tubes (red), and transition elements (green).

turbine, are also logged. They include wind speeds and wind directions (tilt and yaw) at three heights corresponding to the V27 hub height and the lowest and the highest point of the $\mathrm{V} 27$ rotor.

Unfortunately, the trailing edge flap command signal, output from the trailing edge flap controller, was not logged. The trailing edge flap position sensor was however logged.

\subsection{Control system and data acquisition}

The trailing edge flaps controller runs real-time on a NI cRio 9024 real-time controller from National Instruments $^{\mathrm{TM}}$. The controller runs at $800 \mathrm{MHz}$ and has 512 MB DRAM. The NI cRio 9024 sits in the spinner of the turbine. A low-level controller for the trailing edge flaps actuators run on a separate NI cRio 9024. A graphical Labview interface runs on a separate computer.

Logging from the sensors is carried out with several NI cDAQ 9172, sitting in the spinner, the nacelle and next to the met mast.

\section{EXPERIMENTAL METHODS}

\subsection{Open-loop control: sine actuation}

The first tests run on the demonstrator turbine consist in actuating the trailing edge flap as a sine function at the following frequencies: $1 \mathrm{~Hz}, 1.2 \mathrm{~Hz}, 1.6 \mathrm{~Hz}$ and $1.8 \mathrm{~Hz}$. Each test is run during 2 minutes. The first three tests, at $1 \mathrm{~Hz}, 1.2 \mathrm{~Hz}$ and $1.6 \mathrm{~Hz}$ are run at the full trailing edge flap stroke, while the last test, at $1.8 \mathrm{~Hz}$, close to the blade eigenfrequency, is run at a reduced trailing edge flap deflection to avoid a too high excitation of the turbine.

Those tests are meant to assess the dynamics performance of the trailing edge flap, and its effect on the flapwise blade root moment.

\subsection{Close-loop control}

\subsubsection{Trailing edge flap controller target}

The close-loop trailing edge flap controller objective is to reduce flapwise blade root fatigue loads. The equivalent fatigue damage for the blade root is calculated using a rainflow counting algorithm and Miner's linear damage rule [25]. The fatigue damage of a material going through $N_{i}$ cycles of stress range $\sigma_{i}$ is assumed equivalent to the fatigue damage of the same material going throught $N^{e q}$ cycles with a stress range $\sigma^{e q}$, where

$$
\sigma^{e q}=\sum_{i} \sigma_{i}\left(\frac{N_{i}}{N^{e q}}\right)^{1 / m}
$$

An approximation of the Wöhler curve is used to calculate $\sigma^{e q}$. In this paper, $m$, which is a constant specific to the material, is assumed to be 12 for the blade root.

Figure 3 shows the spectral density of the flapwise blade root moment measured on the V27 demonstrator turbine running at its high rotor speed mode. The $1 \mathrm{P}$, $2 \mathrm{P}, 3 \mathrm{P}$ and $4 \mathrm{P}$ frequency loads, corresponding respectively to one, two, three and four events per rotor revolution, are clearly observed, resulting from rotational sampling of turbulence, wind shear etc. Some flapwise loads are also seen at the edgewise eigenfrequency of the blade, because of the coupling of the blade flapwise and edgewise modes. The flapwise eigenfrequency of the modified blade is very close to the $3 \mathrm{P}$ frequency and thus does not show on the spectral density plot as an extra peak. The 1P loads are clearly the cyclic loads with the highest amplitude. They account for a large part of the flapwise blade root fatigue loads. In the example of Figure 3, a flapwise blade root fatigue load reduction of 30 to $50 \%$ could be achieved by alleviating most of the 1P loads. Because of the low control authority of the trailing edge flap, the $1 \mathrm{P}$ loads can not be reduced enough to the point where the $2 \mathrm{P}$ and $3 \mathrm{P}$ loads have a significant part in the flapwise blade root damage. The trailing edge flap controller has then to focus on those $1 \mathrm{P}$ loads first and foremost.

\subsubsection{Measurement methodology}

Evaluating load reduction on a real wind turbine and comparing measurements to simulations is a complex task because of the stochastic nature of the wind, and the lack of information to reproduce the same wind field in simulations. Months of data are usually necessary in order to validate a model. Only few days of measurements, with very different wind conditions, were available within this project. Therefore, it was decided to run the controllers in a burst mode, where 2-minute runs with active trailing edge flap were alternated with 2-minute runs with the trailing edge flap fixed in its neutral position. Two minutes were assumed to be long enough to be able to observe the reponse from the trailing edge flap actuation, and short enough to have similar wind conditions in two consecutive runs. Between 60 and 80 rotor revolutions are observed during two minutes. 


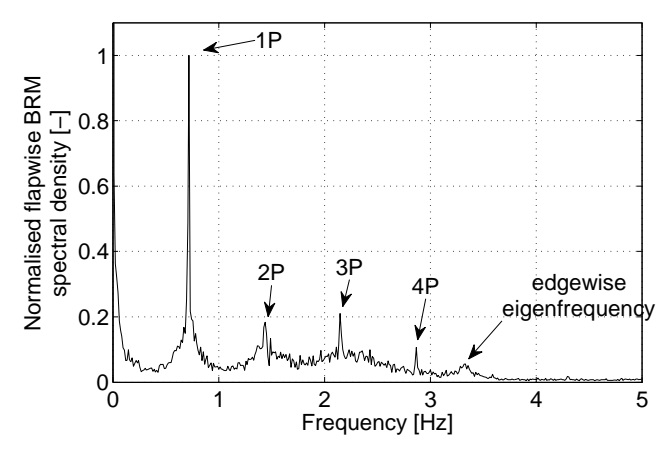

Figure 3. Normalised spectral density of the flapwise blade root moment measured on the V27 test blade. The turbine is in its high rotor speed mode. The $1 \mathrm{P}$ to $4 \mathrm{P}$ loads, corresponding to 1 to 4 events per rotor revolution are clearly identified, as well as loads at the blade edgewise eigenfrequency. The test blade flapwise eigenfrequency is close to the 3P frequency.

\subsubsection{PID controller}

The most basic and natural way to reduce the flapwise blade root fatigue loads is to apply a Proportional-IntegralDerivate controller (PID) to the bandpass filtered flapwise blade root moment. The purpose of the bandpass filter is to highlight the frequency content of the flapwise blade root moment which has to be targeted by the trailing edge flap controller, ie the $1 \mathrm{P}$ to $3 \mathrm{P}$ frequencies. The control law is then

$$
\beta=K_{P} \tilde{M}_{f}+K_{D} \frac{\partial \tilde{M}_{f}}{\partial t}+K_{I} \int \tilde{M}_{f} \mathrm{~d} t
$$

where $\beta$ is the trailing edge flap angle target and $\tilde{M}_{f}$ is the bandpass filtered flapwise blade root moment. $K_{P}, K_{D}$ and $K_{I}$ are respectively the proportional, derivative and integral gains.

Another PID controller is tested, where the controller input is no longer the bandpass filtered flapwise blade root moment but the bandpass filtered pressure difference measured by the leading edge Pitot tube located in front of the trailing edge flap. This pressure difference is correlated to the local angle of attack. The idea behind this controller is to keep the aerodynamic loading constant at the different blade sections equipped with a trailing edge flap, in order to reduce the overall aerodynamic loading variations on the blade, and thus the flapwise blade root fatigue loads.

\subsubsection{Model Predictive Control}

The model predictive control (MPC) tested on the V27 demonstrator turbine is a single input single output frequency-weighted MPC with costs on zero-phase bandpass filtered flapwise blade root moment and on zero-phase bandstop filtered trailing edge flap angle. The bandpass and bandstop filters are tuned in order to alleviate the $1 \mathrm{P}$ and $2 \mathrm{P}$ flapwise blade root loads, and to avoid the trailing edge flap actuation at very low frequencies, and at frequencies higher than the $2 \mathrm{P}$ frequency.

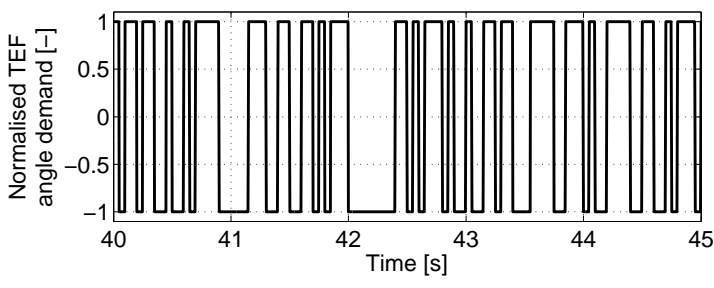

Figure 4. System identification. Trailing edge flap command signal, comprising a Gaussian Binary Noise (GBN) signal.

The design model is a 2-state linear model of a rotating blade, aggregated with disturbance states which model the $1 \mathrm{P}$ and $2 \mathrm{P}$ content of the wind speed seen by the rotating blade, as well as a quasi-steady state which models a plantmodel mismatch. The states are estimated, online, by a predictive Kalman filter. More details on the controller can be found in [17].

Gain scheduling on the mean free wind speed was not possible because of the lack of sensors available to the trailing edge flap controller which could give a good estimate, online, of the mean free wind speed.

\subsection{System identification}

A system identification experiment was performed on the V27 setup using only the outboard flap. A Gaussian Binary Noise (GBN) signal is generated with a sampling frequency of $200 \mathrm{~Hz}$, using a white noise signal sampled with a frequency of $20 \mathrm{~Hz}$, in order to increase the low frequency excitation in expense of the high frequency excitation. The signal comprises in general of random steps at the minimum and the maximum trailing edge flap deflection. The command signal can be seen in Figure 4. This excitation signal is tested during several hours, while the voltage signal from the strain gauge measuring the flapwise blade root strain is simultaneously captured. Tests are run alternating 6 minutes with GBN excitation and 6 minutes without any flap activation. A period of 6 minutes is chosen in order to have enough captured samples of the lower frequencies of interest, and similar wind conditions for consecutive tests.

Then, the input/output data from the GBN excitation cases are used in a subspace system identification algorithm [22] in order to construct a linear state-space model which represents the transfer function between the actual trailing edge flap position and the flapwise blade root moment. Additional periodic inputs at $1 \mathrm{P}$ and $2 \mathrm{P}$ frequencies are added to the input data in order to cope with the periodic dynamics of the system [31]. All the data from the 6 minute cases with flap excitation are tested with the system identification algorithm, using an order number of 20 for the linear models, based on the calculated singular values. The performance of the identification experiment is quantified by looking at the variance-accounted-for (VAF) values. The VAF is defined as: 


$$
\mathrm{VAF}=\max \left\{1-\frac{\operatorname{var}(y-\hat{y})}{\operatorname{var}(y)}, 0\right\} \cdot 100 \%
$$

where $\hat{y}$ is the simulated system output of the identified linear model, and $y$ is the measured system output.

\section{RESULTS OF THE EXPERIMENTS}

Note that measurement results are based on a limited number of tests. Fatigue load reduction is highly dependent on the wind conditions. The load reductions presented in this paper are based on a limited time series and should thus not be considered as the turbines total life fatigue load reduction. Therefore, comparisons between the performance of the controllers have to be made with caution, as the wind conditions changed during the tests of the different controllers.

\subsection{Open-loop control: sine actuation}

The sine actuation tests presented in this section were run when the turbine is in its high rotor speed mode. Tests were run for 2 minutes, for each actuation frequency. The first 10 seconds of each test are removed before postprocessing them, in order to remove any transient.

A clear response in the flapwise blade root moment is seen at the four actuation frequencies (Figure 5). The response in the the flapwise blade root moment is higher if the actuation frequency is close to the first blade eigenfrequency (between 2 and $2.5 \mathrm{~Hz}$ ).

Running these tests gives an estimate of the load alleviation capacity of the trailing edge flap: under those specific wind conditions, the trailing edge flap is able to reduce the amplitude of the 1P flapwise blade root loads by a bit less than $20 \%$.

The spectral densities of the trailing edge flap angle do not show any noise. The actuator is able to produce a clean sine function at the requested frequencies. Only a very small deflection is observed at the $1 \mathrm{P}$ frequency. The same deflection is observed when the trailing edge flap command tries to hold its neutral position.

\subsection{Close-loop control: PID controller}

Tuning a controller in a turbulent wind, when wind conditions change constantly, is a task which requires at least a couple of days. Due to limited time available, only proportional controllers (from now called $\mathrm{P}$ controller) have been tested, with only a rough tuning of the proportional gain and the bandpass filter. The gain tuning was done such that maximum flap angles were reached at the observed range of flapwise blade root moments, while preventing saturation of the flap actuation limits. Those controllers run at a sampling frequency of $200 \mathrm{~Hz}$.
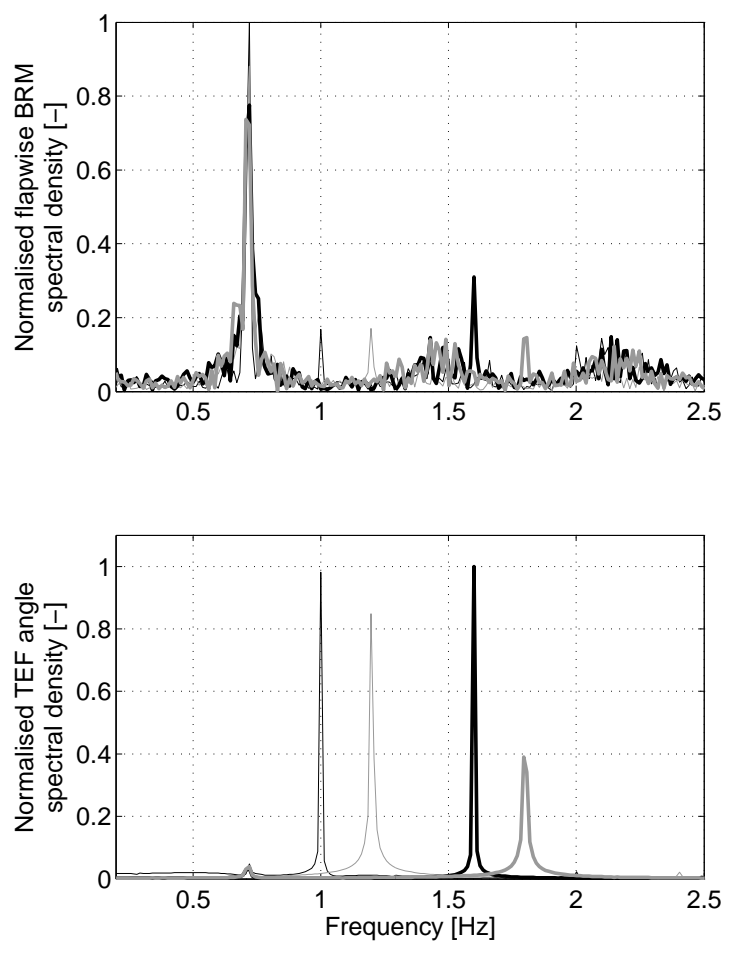

Figure 5. Measurements, sine actuation. Flapwise blade root moment (top) and trailing edge flap angle (bottom) spectral densities when the trailing edge flap is actuated as a sine function at frequencies of $1 \mathrm{~Hz}$ (thin black line), $1.2 \mathrm{~Hz}$ (thin grey line), $1.6 \mathrm{~Hz}$ (thick black line) and $1.8 \mathrm{~Hz}$ (thick grey line).

\subsubsection{Control based on measurement of flapwise blade root moment}

Results showed in Figures 6 and 7 were achieved by running 10 2-minute time series with active trailing edge flap alternating with 10 2-minute time series with a trailing edge flap fixed in its neutral position. Those tests were run with the turbine working in its high rotor speed mode.

Figure 6 shows the spectral densities of the flapwise blade root moment and of the actual trailing edge flap angle averaged over the time series where the trailing edge flap is active or fixed. The trailing edge flap proportional controller reduces slightly the amplitude of the $1 \mathrm{P}$ and $2 \mathrm{P}$ flapwise blade root loads: the 1P load amplitude is decreased by around $10 \%$ and the $2 \mathrm{P}$ load amplitude by around $20 \%$. However, the $\mathrm{P}$ controller increases the $3 \mathrm{P}$ loads as well.

In average, the flapwise blade root fatigue damage is reduced by $4.8 \%$. Figure 7 shows that the trailing edge flap controller decreases the fatigue damage in most of the 2 minute tests, except for the tests number 5, 7 and 17. It is not possible to conclude whether this fatigue damage increase is due to the $\mathrm{P}$ controller increasing the blade root damage, 


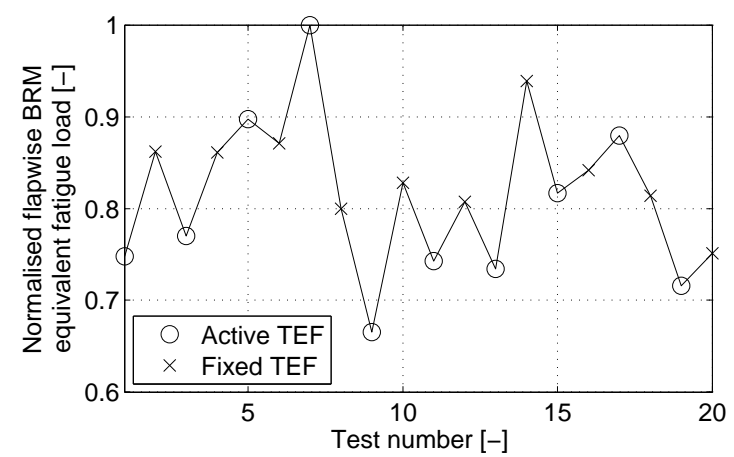

Figure 7. Measurements, proportional controller on flapwise blade root moment. Normalised flapwise blade root equivalent fatigue load for the 20 consecutive 2-minute tests. Tests with fixed trailing edge flaps are marked with crosses, tests with active traiing edge flap are marked with circles.

or to different wind conditions during those 2-minute time series which made the fatigue damage higher than for the previous and the next time series.

The increase of the $3 \mathrm{P}$ loads is responsible for an increase of the flapwise blade root damage. Such an increase could have been avoided by tuning the bandpass filter in order to filter out the flapwise blade root moment frequencies over $1.8 \mathrm{~Hz}$. This tuning was not performed during the tests. However, a post processing of the data shows that a flapwise blade root fatigue damage reduction of 6 to $7 \%$ could have been achieved if the trailing edge flap controller had not increased the $3 \mathrm{P}$ loads.

\subsubsection{Control based on measurement of the local angle of attack}

Only 5 2-minute time series with active trailing edge, alternating with 4 2-minute time series with fixed trailing edge flaps were run with the proportional controller with input from the leading edge pressure difference (Figures 8 and 9). Those tests were run with the turbine running in its high rotor speed mode as well.

The study of the spectral densities of the flapwise blade root moment shows a reduction of the $1 \mathrm{P}, 2 \mathrm{P}$ and $3 \mathrm{P}$ loads (Figure 8). An average fatigue load reduction of $6 \%$ is achieved. However, the fatigue load damage of each of the 9 time series do not show a clear trend of the trailing edge flap reducing the blade root fatigue loads (Figure 9).

The limited number of tests run with this controller makes it hard to draw any conclusions on its performance. The good results seen in the spectral density of the flapwise blade root moment need to be confirmed by running the controller for a longer period of time.

\subsection{Model Predictive Control}

The model predictive control was tested on 10 2-minute time series, alternated with 92 -minute times series with the trailing edge flap fixed in its neutral position. The turbine

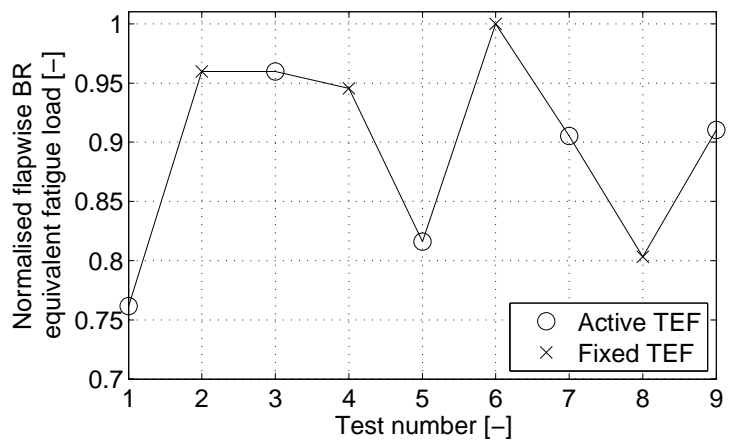

Figure 9. Measurements, proportional controller on the local angle of attack. Normalised flapwise blade root equivalent fatigue load for the 9 consecutive 2-minute tests. Tests with fixed trailing edge flaps are marked with crosses, tests with active traiing edge flap are marked with circles.

was running in its high rotor speed. The controller run at $50 \mathrm{~Hz}$, and was tuned to alleviate the $1 \mathrm{P}$ and $2 \mathrm{P}$ loads only.

Both the $1 \mathrm{P}$ and $2 \mathrm{P}$ loads are reduced, while the $3 \mathrm{P}$ loads are unchanged (Figure 10). The amplitude of the 1P flapwise blade root moment is decreased by $20 \%$. As a comparison, the $\mathrm{P}$ controller with input from the flapwise blade root moment reduced the amplitude of the 1P loads by $10 \%$ (Section 4.2.1).

The study of the equivalent fatigue load of each of the 19 consecutive 2-minute time series shows that the model predictive control consistently reduces the flapwise blade root damage (Figure 11). An average of $14 \%$ load reduction is achieved.

Figure 10 shows that the frequency-weighted model predictive control managed successfully to limit the actuation of the trailing edge flap to frequencies between 0.5 and $2 \mathrm{~Hz}$. There is almost no actuation at frequencies higher than $2.5 \mathrm{~Hz}$, which makes the target signal to the actuator very smooth and thus limits the wear and tear of the actuator, without using any filters on the measurements or on the controller input reference (Figure 12).

\subsection{System identification}

The system identification tests were run while the turbine was in its low rotor speed mode, contrary to the active load reduction tests which were run at high rotor speed.

Because of the dynamics of the actuator the GBN command signal is not perfectly followed by the flap, showing a resulting flap signal as seen in Figure 13. Despite the distorted nature of the GBN signal, it can still provide enough excitation to the system. The captured strain signals for all cases with GBN flap excitation are compared to the ones without flap activation in terms of spectral content, but no conclusive effect of the flap excitation is visible.

VAF values of $37 \%$ to $65 \%$ were obtained on the various data sets, which were in this case the same as the ones used for determining the model. For the best case with the highest 

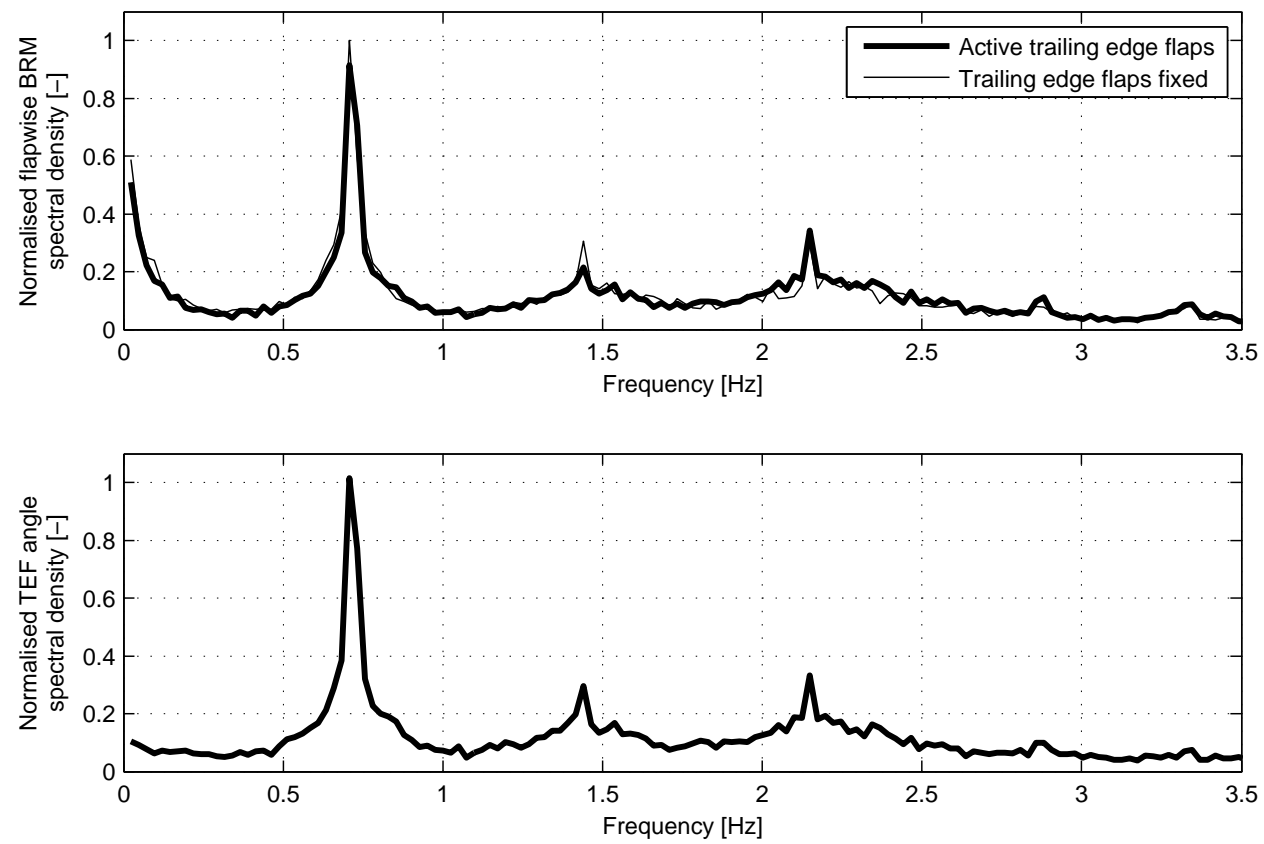

Figure 6. Measurements, proportional controller on flapwise blade root moment. Flapwise blade root moment (top) and trailing edge flap angle (bottom) spectral densities when the trailing edge flaps are fixed in their neutral position (thick black line) and when they are controlled with a proportional controller on the flapwise blade root moment (thin black line).

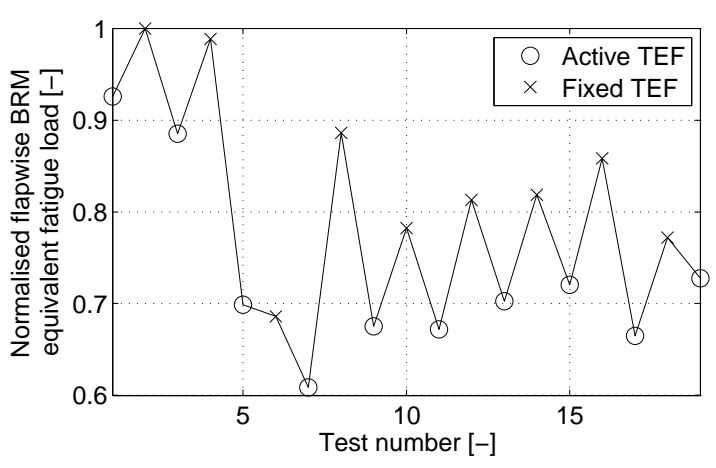

Figure 11. Measurements, model predictive control. Normalised flapwise blade root equivalent fatigue load for the 19 consecutive 2-minute tests. Tests with fixed trailing edge flaps are marked with crosses, tests with active traiing edge flap are marked with circles.

match, the output of the simulation of the identified linear model to the measured input is compared to the measured system output. The de-trended output signals are shown in Figure 14. The frequency response plot of the amplitude of the input-output behavior is also compared to the measured spectrum in Figure 15. It is seen that the linear model captures the global low frequency behavior, but also the
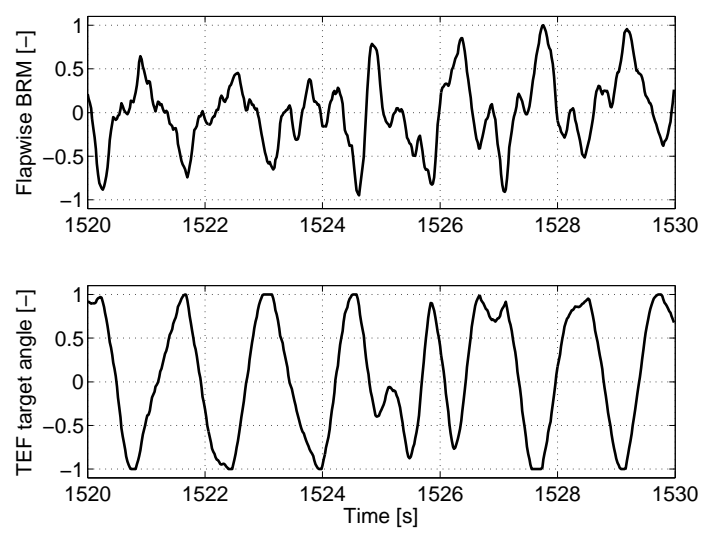

Figure 12. Measurements, model predictive control. Normalised flapwise blade root moment (top) and controller input reference (trailing edge flap target angle) measured when the model predictive control is running on the V27.

natural frequency peaks of the system. The $1 \mathrm{P}$ and $2 \mathrm{P}$ peaks are intentionally not captured, due to additional periodic inputs in the algorithm, in order to capture a linear time invariant model [31]. 

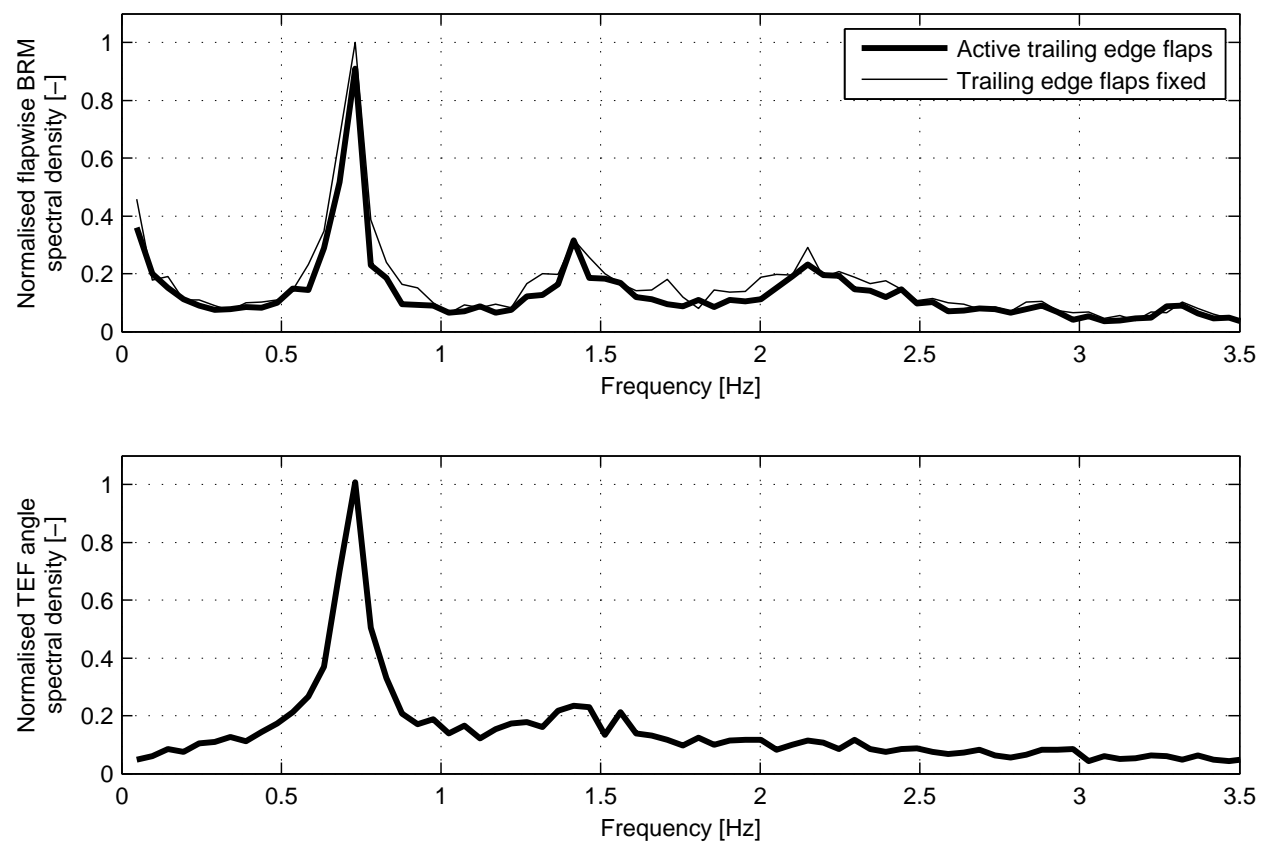

Figure 8. Measurements, proportional controller on the local angle of attack. Flapwise blade root moment (top) and trailing edge flap angle (bottom) spectral densities when the trailing edge flaps are fixed in their neutral position (thick black line) and when they are controlled with a proportional controller on the local angle of attack (thin black line).

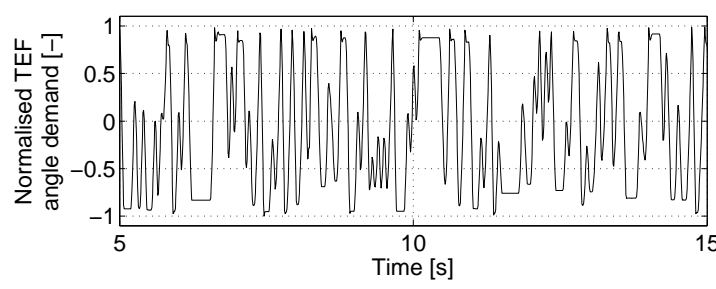

Figure 13. System identification. Actual flap angle response to the GBN command signal.

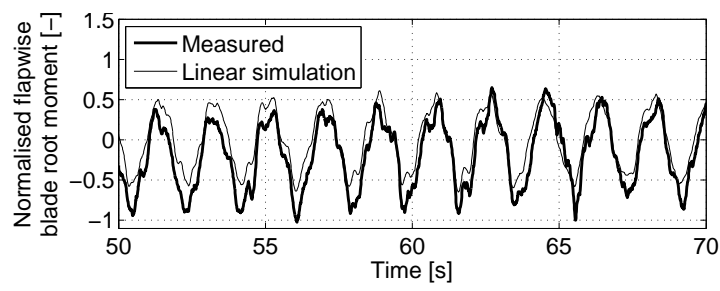

Figure 14. System identification. Time series of blade root strain signal for flap-wise bending. The output of the linear simulation of the identified model (thin line) is compared to the measured signal (thick line).

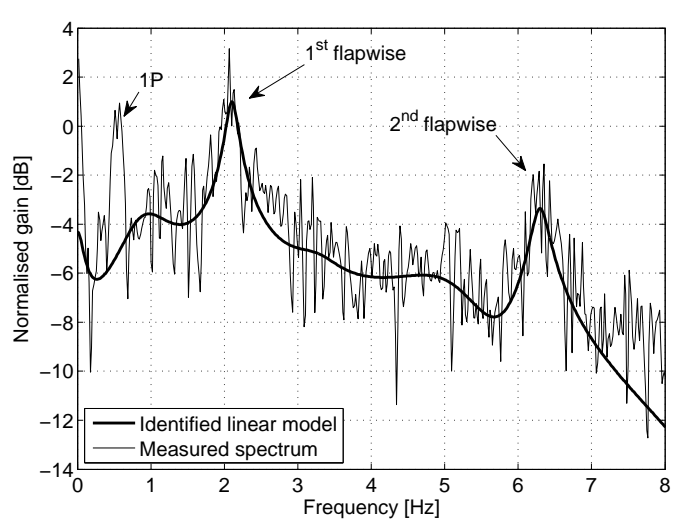

Figure 15. System identification. Frequency response from actual flap signal to blade root strain. The identified model (thick line) is compared to the spectral estimate of the measured signal (thin line)

\section{COMPARISON WITH SIMULATIONS}

Simulations with the sine actuation of the trailing edge flap and with the model predictive control are run and compared to the measurements. 

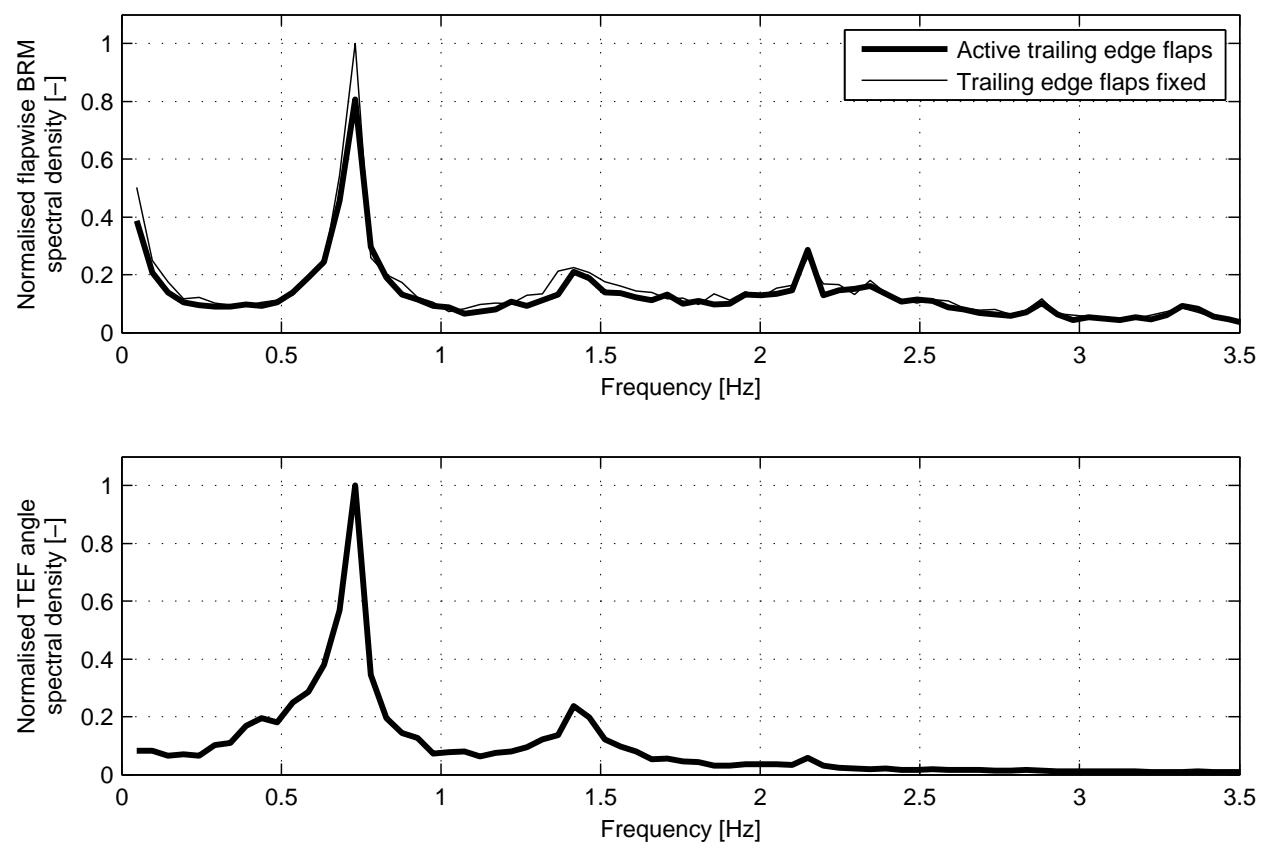

Figure 10. Measurements, model predictive control. Flapwise blade root moment (top) and trailing edge flap angle (bottom) spectral densities when the trailing edge flaps are fixed in their neutral position (thick black line) and when they are controlled with a model predictive control (thin black line).

\subsection{Simulation models}

\subsubsection{Aero-servo-elastic code}

Simulations are run with the Flex 5 aero-servo-elastic code [27]. Flex5 is developed at the Mechanical department of the Technical University of Denmark DTU-MEK. It is a state-of-the art Blade Element Momentum (BEM) code based on the modal approach. The blades, the shaft and the tower are modeled as flexible non uniform beams. The code has all the usual engineering models used in the wind turbine simulation tools, such as Prandtl-Glauert tip correction, dynamic wake model, turbulent wind, oblique inflow model etc.

The dynamic stall model originally coded in Flex 5 works only for rigid blade sections [26]. The implementation of the trailing edge flaps in Flex 5 is based on the code written by P. B. Andersen and M. Gaunaa [19]. It is an extension of Theodorsen's work [30] which includes a BeddoesLeishmann type dynamic stall model [24]. The model uses lookup tables of the static lift, drag and moment coefficients of an aerofoil with a trailing edge flap at different angles of attack and trailing edge flap angles. The model has been validated with comparison to wind tunnel measurements [3].

\subsubsection{Wind turbine model}

The Flex5 wind turbine model was derived from a previously existing Flex4 model and from technical documents. This model was partly tuned based on the measurements made during the first test campaign of the ATEF project, on the same V27 wind turbine [18]. However, this Flex 5 model has never been validated and differences between the measurements and the simulations can be observed.

An important part of the model of the V27 wind turbine with trailing edge flaps is the steady-state lift coefficient change due to the trailing edge flap $\Delta C_{L}^{\text {st }}(\alpha, \beta)$ defined as

$$
\Delta C_{L}^{\mathrm{st}}(\alpha, \beta)=C_{L}^{\mathrm{st}}(\alpha, \beta)-C_{L}^{\mathrm{st}}(\alpha, \beta=0),
$$

where $C_{L}^{\text {st }}(\alpha, \beta)$ is the steady lift coefficient of the aerofoil, at an angle of attack $\alpha$ and a trailing edge flap angle $\beta$. An array of $\Delta C_{L}^{\text {st }}(\alpha, \beta)$ values, for different angles of attack and trailing edge flap angles, was filled in based on CFD simulations with a similar aerofoil and trailing edge flap.

\subsection{Sine actuation}

Some Flex 5 simulations are run with the outer trailing edge flap being actuated as a sine function with the same frequencies and amplitudes as the ones used during the experiment. Figure 16 shows a zoom on the four actuation frequencies of the spectral densities of the flapwise blade root moment and of the trailing edge flap angle.

The simulations (Figure 16, dashed lines) show a higher response on the blade root moment than what is seen in the 
measurements (Figure 16, solid lines, no markers), at the four actuation frequencies. The amplitudes of the simulated flapwise blade root moments, at the actuation frequencies, are around 30\% higher than in the measurements. This significant difference can be explained by an overestimate of the steady lift coefficient change due to a fixed trailing edge flap angle $\Delta C_{L}^{\text {st }}(\alpha, \beta)$, by 3D effects at the trailing edge flap edges which could decrease the trailing edge flap efficiency [8], or, most probably, by a combination of both reasons. The uncertainty on the steady lift coefficient change could be reduced by performing wind tunnel tests with the same aerofoil profile and the same trailing edge flap.

Another set of simulations were run with a trailing edge flap efficiency reduced by $20 \%$ (Figure 16, solid lines with cross markers). The blade root response in those simulations is closer to the measurements. It is still a bit too high for the 1 and $1.2 \mathrm{~Hz}$ actuation cases, but matches well the measurements for the two highest actuation frequencies. An overestimate of the blade damping, either structural or aerodynamic damping, could explain why the simulations match the measurements at higher frequencies but they overestimate the flapwise blade root response at lower frequencies.

\subsection{Model Predictive Control}

Exactly the same model predictive controller, with the same tuning, the same sampling frequency and the same sensors as the one used in the experiment is run in Flex 5 simulations. In order to reproduce the test conditions in the simulations, the pitch position sensor is assumed not to work, and gain scheduling is not used. When the field tests were run, the flapwise blade root strain gauge had been only roughly calibrated. Calibration after the tests showed a small error in the strain gauge calibration gain. The same error was modeled in the simulations.

An average wind speed, wind turbulence intensity, wind shear and yaw error were calculated from the measurements, and Flex5 simulations with the same wind conditions and yaw error were run with six different wind turbulence boxes. However, those simulations resulted in 1P flapwise blade root moments with a significantly lower amplitude than in the measurements. The wind shear and yaw error have then been increased until the $1 \mathrm{P}$ peak in the spectral density of the flapwise blade root moment in the simulations and the measurements match (Figure 17). The $3 \mathrm{P}, 4 \mathrm{P}$ and $5 \mathrm{P}$ peaks are higher in the simulations than in the measurements. However, the load reduction happens at the $1 \mathrm{P}$ and $2 \mathrm{P}$ frequencies, so it is more important than the loads at those freuqencies match.

A large difference in the simulated and measured equivalent fatigue loads, with fixed trailing edge flaps, is observed: the blade root fatigue loads simulated in Flex 5 are $14.5 \%$ lower than in the measurements. If only the $1 \mathrm{P}$ and $2 \mathrm{P}$ loads are taken into account, the difference raises to $24 \%$. It is thought that such a difference is due to difference in the simulated wind: the wind shear, within a 2-minute test, varies significantly more in the measuremetns than in the simulations.

Figure 18 shows the spectral density of the flapwise blade root moment and of the trailing edge flap angle of Flex 5 simulations and measurements, with active trailing edge flaps. The simulated spectral densities with fixed trailing edge flaps are also plotted as a reference. Figure 19 shows a zoom of the previous plots around the $1 \mathrm{P}$ and the $2 \mathrm{P}$ frequencies. Results from Flex 5 simulations run with a trailing edge flap efficiency reduced by $20 \%$ are also plotted (dashed lines with triangle markers). Those plots show a general good agreement between the simulations and the measurements. The trailing edge flap actuation is similar in measurements and in simulations. Note that the difference in the $1 \mathrm{P}$ peak of the trailing edge flap angle spectral density is not due to different amplitudes, but to slightly different shapes of the trailing edge flap angle time track. The reduction of the $1 \mathrm{P}$ peak in the flapwise blade root moment spectral density is higher in the simulations made with a trailing edge flap efficiency of $100 \%$ than in the measurements. But the results for a trailing edge flap efficiency of $80 \%$ match very well (Figure 19).

The difference in terms of flapwise blade root fatigue load reduction between measurements and simulations are higher. Measurements showed a load reduction of $14 \%$, whereas simulations show a load reduction of $5.9 \%$ with a trailing edge flap efficiency of $100 \%$ and of only $4.7 \%$ with a trailing edge flap efficiency of $80 \%$. However, the difference is lower if only the $1 \mathrm{P}$ and $2 \mathrm{P}$ loads are taken into account: $20 \%$ load reduction in measurements, and $12.4 \%$ load reduction in Flex5 simulations with a trailing edge flap efficiency of $80 \%$.

\subsection{System identification}

The linear model from trailing edge flap angle to flapwise blade root moment estimated with the system identification described in Section 4.4 is compared to the analytical linear model derived in [17] and part of the MPC design model. Figure 20 shows the Bode plots of the 20-state linear model derived with system identification, of the 2-state analytical linear model part of the MPC design model and of the same model tuned in order to match the conclusions of the experiment: the trailing edge flap efficiency is reduced by $20 \%$, and the blade stiffness is reduced until the blade first eigenfrequency match the measured eigenfrequency (around the 3P frequency). The response from the trailing edge flap sine actuation, at both low and high rotor speeds, is also plotted.

Figure 20 shows a significant difference in the first blade eigenfrequency between the identified model and the analytical model. The mass distribution and the stiffness distribution of the modified blade were only roughly estimated, which explains this large difference. The analytical tuned model still differs largely from the identified model at the frequencies of interest for the model predictive control $(0.3$ to $2 \mathrm{~Hz})$, partly because a VAF value of only $65 \%$ was reached when performing the system 

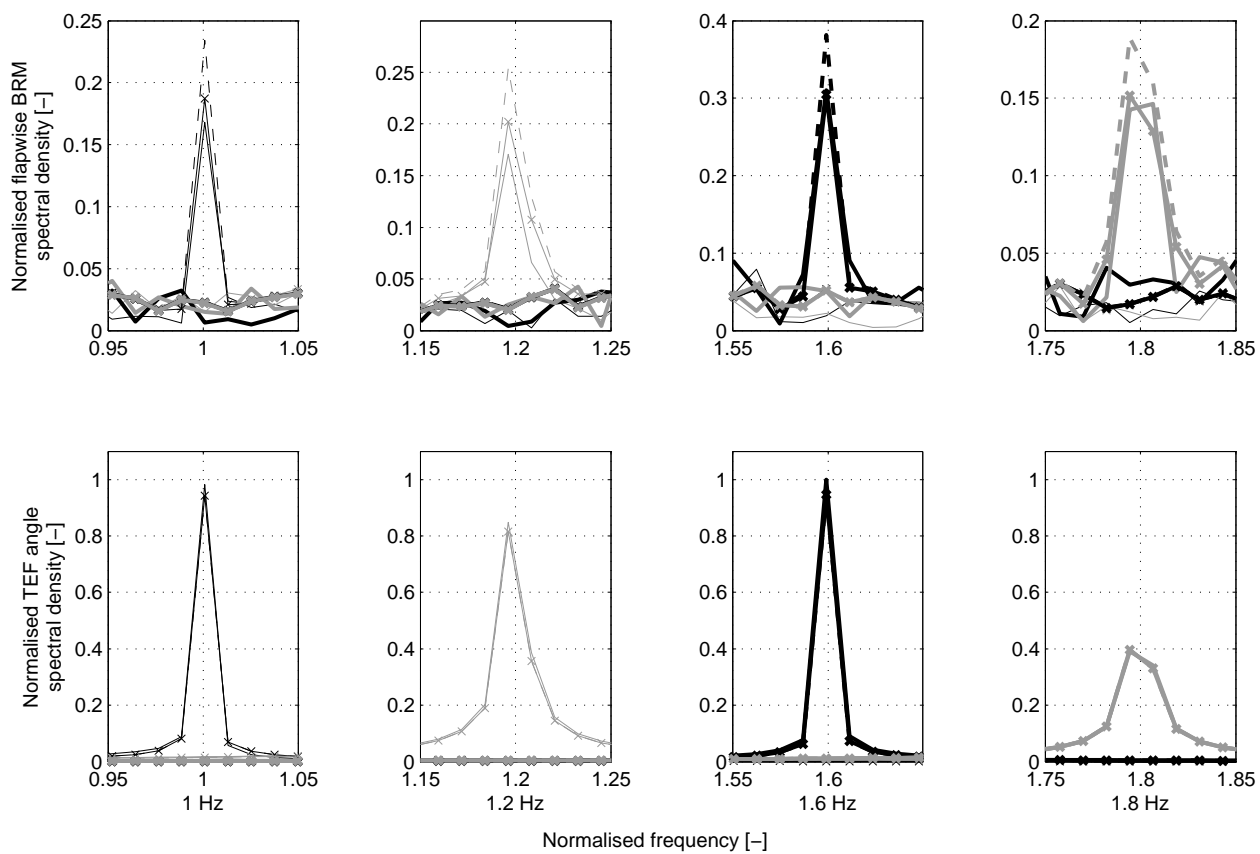

Figure 16. Measurements and simulations, sine actuation. Zooms on the flapwise blade root moment (top) and trailing edge flap angle (bottom) spectral densities when the trailing edge flap is actuated as a sine function at frequencies of $1 \mathrm{~Hz}$ (thin black line), $1.2 \mathrm{~Hz}$ (thin grey line), $1.6 \mathrm{~Hz}$ (thick black line) and $1.8 \mathrm{~Hz}$ (thick grey line). Results from measurements (solid lines), from simulations with a trailing edge flap efficiency of $100 \%$ (dashed lines) and from simulations with a reduced trailing edge flap efficiency (solid lines with crosses).

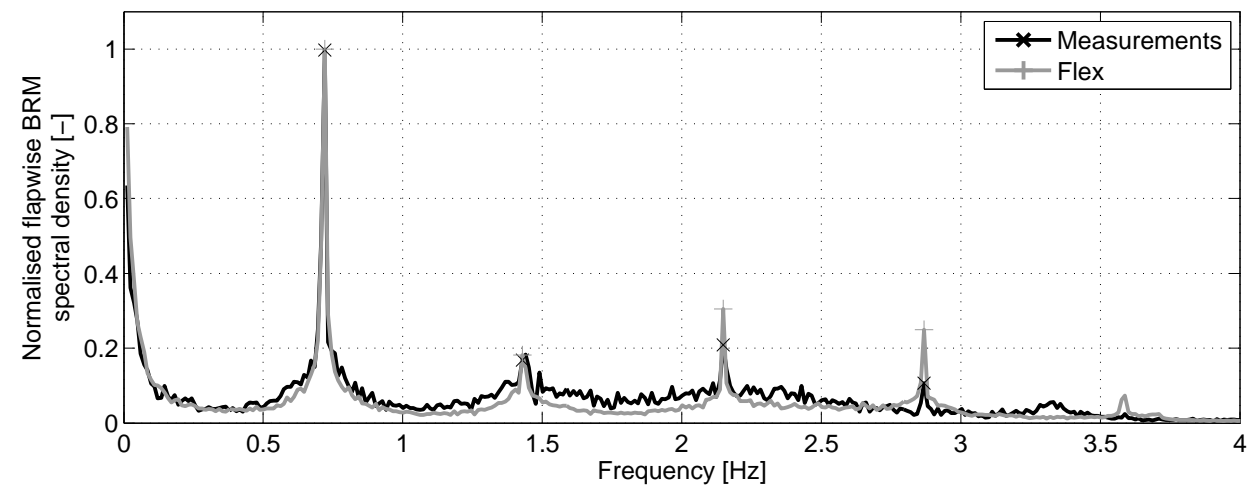

Figure 17. Measurements and simulations, model predictive control. Measured (black line with crosses) and simulated (grey line with +) flapwise blade root moment spectral densities when the trailing edge flaps are fixed in their neutral position.

identification, and partly because the analytical model probably overestimates the trailing edge flap efficiency when the turbine runs in its low rotor speed mode.

\section{CONCLUSIONS}

A full-scale test of trailing edge flaps on the Vestas V27 wind turbine was performed. Even though only $5 \%$ of the blade span was covered with a working trailing edge flap, clear load reduction could be observed.

Three active load reduction controllers were tested. The two proportional controllers, respectively on the flapwise 

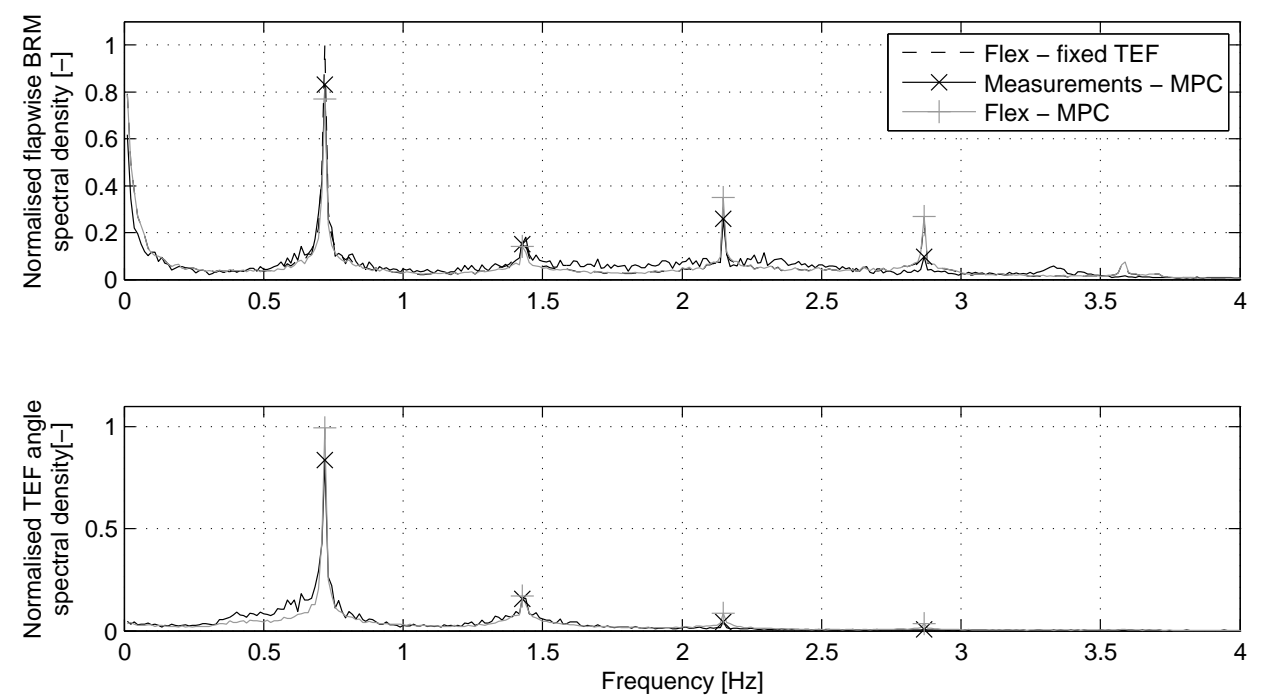

Figure 18. Measurements and simulations, model predictive control. Measured (black line with crosses) and simulated (grey line with + ) flapwise blade root moment (top) and trailing edge flap angle (bottom) spectral densities when the trailing edge flaps are controlled with a frequency-weighted model predictive control. Spectral densities for the simulated baseline case with fixed trailing edge flaps is plotted for comparison (dashed line).
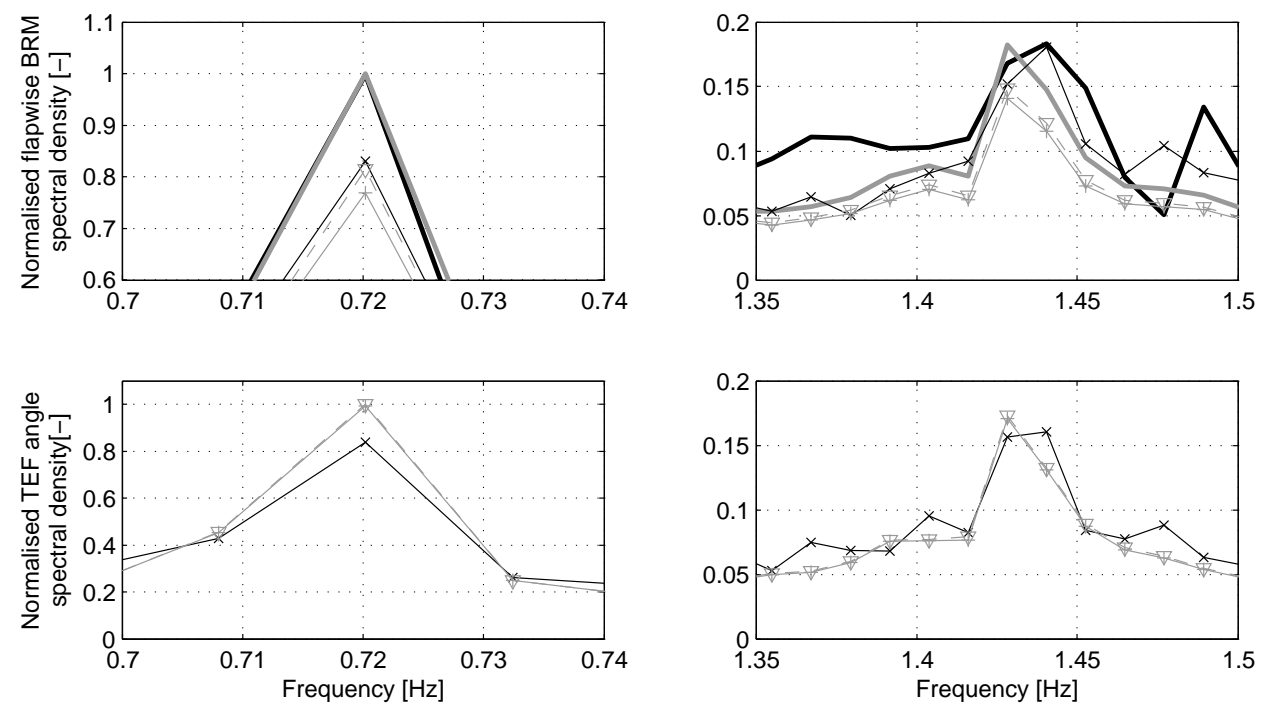

Figure 19. Measurements and simulations, model predictive control. Zooms on the $1 \mathrm{P}$ and $2 \mathrm{P}$ frequencies. Measured and simulated flapwise blade root moment (top) and trailing edge flap angle (bottom) spectral densities when the trailing edge flaps are controlled with a frequency-weighted model predictive control and when they are fixed. Thick black line: measurements, baseline. Thick grey line: simulations, baseline. Thin black line with crosses: measurements, MPC. Thin grey line with + : simulations, MPC. Dashed grey line with triangles: simulations with reduced trailing edge flap efficiency, MPC.

blade root moment and on the local angle of attack at the trailing edge flap section, reduced slightly the amplitude of the $1 \mathrm{P}$ and $2 \mathrm{P}$ flapwise blade root loads. A flapwise blade root load reduction between 4 and $6 \%$ was measured. More tests would be necessary to confirm those results, and it is thought that higher load reduction would be achieved with a better tuning of the controllers. A frequency-weighted model predictive control tested during 38 minutes showed 

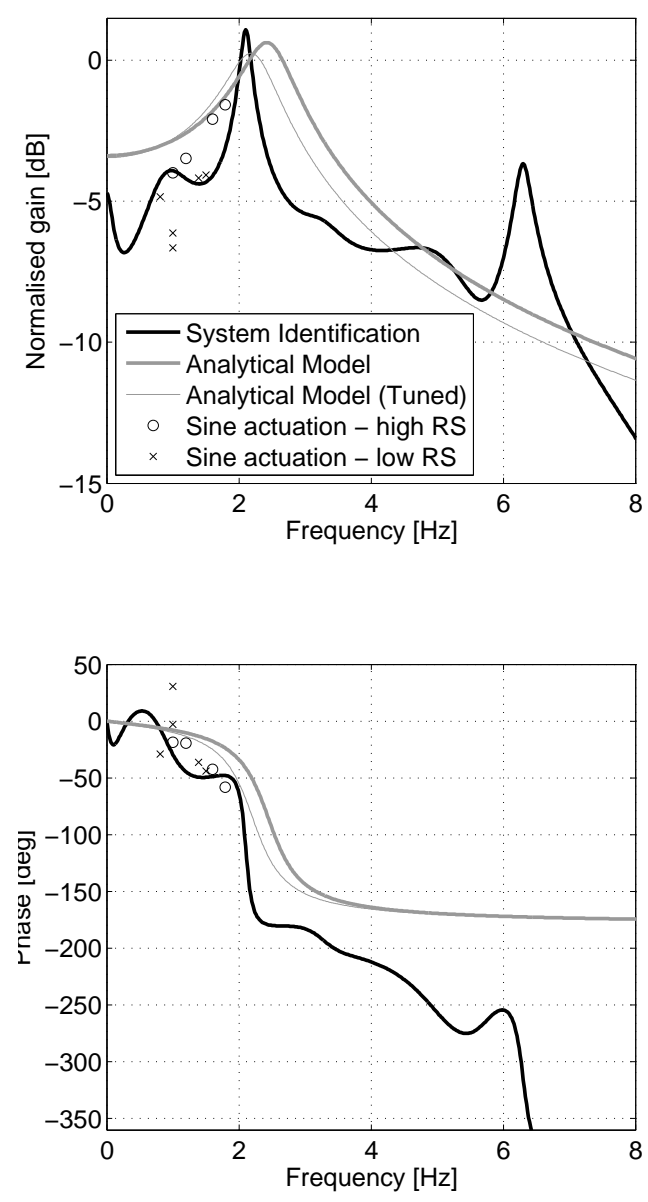

Figure 20. Bode plots from actual trailing edge flap angle position to flapwise blade root moment, for the identified model (thick black line), the analytical model used in the design of the model predictive control (thick grey line), and for a tuned analytical model where the trailing edge flap efficiency has been reduced by $20 \%$ and the blade mass and stiffness distribution have been modified in order to decrease the blade first flapwise eigenfrequency (thin grey line). The responses from a sine actuation of the trailing edge flap, at different frequencies, at the high rotor speed (circles) and at the low rotor speed (crosses) are also plotted.

consistent flapwise blade root load reduction with the trailing edge flap. An average of $14 \%$ load reduction was measured, and a $20 \%$ reduction of the amplitude of the $1 \mathrm{P}$ loads was observed.

System identification was also tested on the demonstrator turbine. A 20 state time invariant linear model was derived with a variance-accounted-for value of $65 \%$.

Finally, Flex5 simulations were run to compare the simulation models to the measurements. A reduction of
$20 \%$ of the trailing edge flap efficiency was necessary to match the simulation results with the measurements. It was however not possible to conclude whether the reduced efficiency was due to $3 \mathrm{D}$ effects at the trailing edge flap edges, or to overestimated steady-state lift coefficient changes due to the trailing edge flap. The simulations and the measurements carried out with exactly the same model predictive control showed very good correlation in their spectral densities. A $14 \%$ flapwise blade root load reduction was measured, whereas only a $5 \%$ load reduction was simulated. The difference is explained partly by the difference in the fatigue loads with fixed trailing edge flaps.

This new full-scale test on the Vestas V27 wind turbine equipped with trailing edge flaps proved the load alleviation capability of the trailing edge flaps. They also showed a good accuracy of the simulations. However, more tests, with different wind conditions, would be necessary to conclude on the amount of load reduction achievable with this trailing edge flap configuration.

\section{ACKNOWLEDGEMENTS}

The authors would like to thank the Danish National Advanced Technology Foundation (Højteknologifonden) for partially funding this work, as part of the ATEF (Adaptive Trailing Edge Flaps) project.

The results presented in this paper were made possible thanks to the involvement and the commitment of many colleagues from the Test and Measurements group and the Aeroelastic design groups at Risø DTU, National Laboratory for Sustainable Energy, and from the Mechatronics group of Vestas Wind Systems A/S, Singapor. The authors would like to acknowledge especially Anker Bruun Andersen, Kasper Clemmensen, Karen Enevoldsen, Per Hansen, René Kjærsgaard, Michael Rasmussen and Allan Vesth.

\section{REFERENCES}

1. P. B. Andersen, C. Bak, M. Gaunaa, and T. Buhl. Wind tunnel test of a closed loop controller for an airfoil with trailing edge flaps. In TORQUE 2010: The Science of Making Torque from Wind, Crete, Greece, June 2010.

2. Peter Bjoern Andersen, Lars Henriksen, Mac Gaunaa, Christian Bak, and Thomas Buhl. Deformable trailing edge flaps for modern megawatt wind turbine controllers using strain gauge sensors. Wind Energy, 13(2-3):193-206, 2010.

3. Peter Bjrn Andersen, Mac Gaunaa, Christian Bak, and Morten Hartvig Hansen. A dynamic stall model for airfoils with deformable trailing edges. Wind Energy, 12(8):734-751, 2009.

4. T. D. Ashwill, G. Kanaby, K. Jackson, and M. Zutech. Development of the swept twist adaptive rotor (star) blade. In Proceedings of the 48th AIAA Aerospace 
Sciences Meeting Including the New Horizons Forum and Aerospace Exposition, Orlando, Florida, 2010.

5. Christian Bak, Mac Gaunaa, Peter B. Andersen, Thomas Buhl, Per Hansen, and Kasper Clemmensen. Wind tunnel test on airfoil Ris $\varnothing$-B1-18 with an active trailing edge flap. Wind Energy, 13(2-3):207-219, 2010.

6. TK Barlas and GAM van Kuik. Review of state of the art in smart rotor control research for wind turbines. Progress in Aerospace Sciences, 46(1):1-27, 2010.

7. S. Basualdo. Load alleviation on wind turbine blades using variable airfoil geometry. Wind Engineering, 29(2):169-182, Mar. 2005.

8. T. Behrens. Simulation of moving trailing edge flaps on a wind turbine blade using a navier-stokes based immersed boundary method. PhD thesis [to be published].

9. T. Behrens and W. Jun Zhu. Feasibility of aerodynamic flap hinge moment measurements as input for load alleviation control. In EWEA 2011, Brussels, Belgium, 2011.

10. E. A. Bossanyi. Individual blade pitch control for load reduction. Wind Energy, 6(2):119-128, 2003.

11. E. A. Bossanyi. Further load reductions with individual pitch control. Wind Energy, 8(4):481-485, 2005.

12. T. Buhl, M. Gaunaa, and C. Bak. Potential load reduction using airfoils with variable trailing edge geometry. Journal of Solar Energy Engineering, 127(4):503-516, November 2005.

13. T. Burton, D. Sharpe, N. Jenkins, and E. A. Bossanyi. Wind Energy handbook. Wiley, 2008.

14. M. Capellaro and M. Kühn. Boundaries of bend twist coupling. In TORQUE 2010: The Science of Making Torque from Wind, 2010.

15. D. Castaignet, L. Bergami, T. Buhl, N. K. Poulsen, and J. J. Wedel-Heinen. Robustness assessment of a flap controller with two alternative aeroelastic simulation tools. [submitted].

16. D. Castaignet, T. Buhl, N. K. Poulsen, and J. J. WedelHeinen. Trailing edge flaps impact on fatigue and extreme loads in power production. In EWEA 2011, Brussels, Belgium, 2011.

17. D. Castaignet, I. Couchman, N. K. Poulsen, T. Buhl, and J. J. Wedel-Heinen. Frequency-weighted model predictive control of trailing edge flaps on a wind turbine blade. [submitted].

18. D. Castaignet, J. J. Wedel-Heinen, T. Kim, T. Buhl, and N. K. Poulsen. Results from the first full scale wind turbine equipped with trailing edge flaps. In 28th AIAA Applied Aerodynamics Conference, Chicago, Illinois, June 2010.

19. M. Gaunaa. Unsteady two-dimensional potential-fl ow model for thin variable geometry airfoils. Wind Energy, 13:167-192, 2010.

20. M. H. Hansen. Aeroelastic modal analysis of backward swept blades using HAWCStab2. Technical
Report Ris $\emptyset-R-1769(E N)$, Ris $\emptyset$ DTU, National Laboratory for Sustainable Energy, February 2011.

21. M. H. Hansen, A. Hansen, T. J. Larsen, S. Øye, P. Sørensen, and P. Fuglsang. Control design for a pitch-regulated, variable speed wind turbine. Technical Report Risø-r-1500(EN), Risø National Laboratory, January 2005.

22. I. Houtzager, J.-W. van Wingerden, and M. Verhaegen. Predictor-based subspace identification toolbox version 0.4, 2010. http://www.dcsc.tudelft.nl/ datadriven/pbsid/.

23. Torben Juul Larsen, Helge A. Madsen, and Kenneth Thomsen. Active load reduction using individual pitch, based on local blade flow measurements. Wind Energy, 8(1):67-80, 2005.

24. J. G. Leishman. Unsteady lift of a flapped airfoil by indicial concepts. Journal of Aircraft, 31(2):288-297, March-April 1994.

25. J. F. Manwell, J.G. McGowan, and A. L. Rogers. Wind energy explained. Theory, Design and Application. John Wiley \& Sons Ltd, 2008. ISBN: 13:978-0-47149972-5.

26. S. Øye. Dynamic stall simulated as time lag of separation. In EWEC 1994, Thessaloniki, Greece, October 1994.

27. S. Øye. Flex 4 - Simulation of wind turbine dynamics. In Proceedings of the $28^{\text {th }}$ IEA Meeting of Experts "State of the Art of Aeroelastic Codes for Wind Turbine Calculations, pages 71-76, Technical University of Denmark, Lyngby, Denmark, April 11121996.

28. J. A. Paquette and P. S. Veers. Increased rotor size through passive load control and weight reduction concepts. In EWEC 2009, Marseille, France, 2009.

29. K. Selvam, S. Kanev, J. W. van Wingerden, T. van Engelen, and M. Verhaegen. Feedbackfeedforward individual pitch control for wind turbine load reduction. International Journal of Robust and Nonlinear Control, 19(1):72-91, 2009.

30. T. Theodorsen. General theory of aerodynamical instability and the mechanism of flutter. Technical Report Report 496, NACA, 1935.

31. G. J. Van der Veen, J.-W. van Wingerden, and M. Verhagen. Closed-loop system identification of wind turbines in the presence of periodic effects. In $3^{\text {rd }}$ conference on the science of making torque from wind, Heraklion, Greece, 2010.

32. J.-W. van Wingerden, A. Hulskamp, T. Barlas, I. Houtzager, H. Bersee, G. van Kuik, and M. Verhaegen. Two-degree-of-freedom active vibration control of a prototyped smart rotor. Control Systems Technology, IEEE Transactions on, 19(2):284 -296, march 2011.

33. J. W. van Wingerden, A. W. Hulskamp, T. Barlas, B. Marrant, G. A. M. van Kuik, D.-P. Molenaar, and M. Verhaegen. On the proof of concept of a smart wind turbine rotor blade for load alleviation. Wind Energy, 11(3):265-280, 2008. 